\title{
Driver Lower Extremity Response and Injury with Knee Airbag Deployment
}

\author{
A Thesis Presented to the faculty of \\ The School of Engineering and Applied Science \\ University of Virginia
}

\begin{abstract}
In Partial Fulfillment
of the Requirements for the Degree

Master of Science in Mechanical and Aerospace Engineering

by
\end{abstract}

$\mathrm{Xin} \mathrm{Ye}$

Aug 2015 


\section{APPROVAL SHEET}

The thesis is submitted in partial fulfillment of the requirements for the degree of Master of Science (Mechanical and Aerospace Engineering)

Xin Ye

Author

The thesis has been read and approved by the examining Committee:

Jeff R. Crandall

Thesis Advisor

Richard W. Kent

Committee Chair

Matthew B. Panzer

Committee Member

Accepted for the School of Engineering and Applied Science:

James H. Aylor

Dean, School of Engineering and

Applied Science

Aug 2015 


\section{ACKNOWLEDGMENTS}

I would like to first thank my advisor, Jeff Crandall, who accepted me as a graduate student and opened the door of biomechanics for me. Dr. Crandall taught me everything about injury biomechanics, and most importantly, guided me the way to develop the critical thinking in doing research.

I would like to thank all the students and staff at Center for Applied Biomechanics of University of Virginia, who made the past four years the best time of my life. Special acknowledgment goes to Matt Panzer and Greg Shaw for their tireless work on the knee airbag project. Also, I wish to acknowledge Gerald Poplin, Lee Gabler, Bingbing Nie, Taewung Kim, Jim Funk for all the technical help provided, and always be there as a friend to talk to.

Finally, I would like to thank my parents for their love and support. Their encouragement helps me to remain hopeful and optimistic along the way. 


\section{ABSTRACT}

Lower extremity injury is the leading AIS2+ injury for drivers involved in frontal crashes. While there has been steady improvement in vehicle crash test performance over the past two decades, the risk of lower extremity injury remains virtually unchanged.

As one potential injury countermeasure, knee airbags and inflatable knee bolsters have been purportedly designed to mitigate lower limb forces and to improve overall occupant safety by providing early restraint for the pelvis and lower torso. Recent studies, however, suggest an increased injury risk of the tibia/fibula and the foot/ankle with knee airbags. This thesis aims to investigate the lower extremity response and injury with knee airbag deployment, and to identify any adverse effects the knee airbag imposes on lower extremity.

The thesis examines the lower extremity response for drivers in nominal driving posture, under dynamic condition with the presence of an Inflatable Knee Bolster (IKB). A total of seven unbelted sled tests $(40 \mathrm{~km} / \mathrm{h})$ and eight belted sled tests $(56 \mathrm{~km} / \mathrm{h})$ were performed with a $50^{\text {th }}$ percentile Hybrid-III dummy to investigate the IKB effects on the lower limb. For the unbelted tests, the average tibia index (TI)increased by $34.6 \%$ and $33.3 \%$ for tests with IKB on the left upper $(1.10 \pm 0.10)$ and left lower

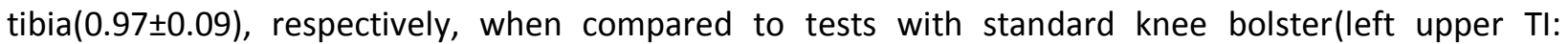
$0.82 \pm 0.11$; left lower TI: $0.73 \pm 0.05$ ). Additionally, the right upper tibia index increased by $20.3 \%$ (from $0.64 \pm 0.10$ to $0.77 \pm 0.04$ ), while the right lower tibia index decreased by $26.5 \%$ (from $0.86 \pm 0.12$ to $0.63 \pm 0.04)$. Similar results were found for the belted tests with IKB, where a $9.1 \%$ increase in risk of AIS $2+$ tibia shaft fractures (from $11.8 \%$ to $20.9 \%$ ) was predicted with IKB deployment.

The study also investigated the effects of the knee airbag on the lower limb injury risk for out-ofposition drivers. A total of eleven static knee airbag deployment tests were performed using a $5^{\text {th }}$ percentile female Hybrid-III dummy outfitted with either the original Hybrid-III lower extremities or the $5^{\text {th }}$ percentile THOR-FLx. Baseline tests were performed with FMVSS 208 seating specifications, and a design of experiment for out-of-position conditions was developed with multiple factors including kneeto-instrument panel distance, knee-to-knee distance, and foot placement. The results predicted a $40.6 \%$ increase in risk of AIS 2+ tibia shaft fractures for out-of-position drivers (Ave. 53.6\%) relative to inposition drivers (Ave. 13.0\%).

This thesis indicates that inflatable knee bolsters can increase the likelihood of drivers sustaining tibia/fibula injuries in frontal crashes. In addition, the dummy lower extremity responses recorded in 
static deployment tests suggest a high risk of lower extremity injury for both in-position and out-ofposition small female drivers during knee airbag deployment. While this thesis covers a limited set of crash conditions and knee airbag designs, the results indicate the need for improved design of knee airbag for better protection of lower limb. 


\section{Table of Contents}

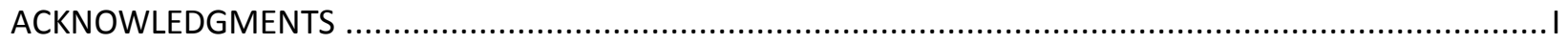

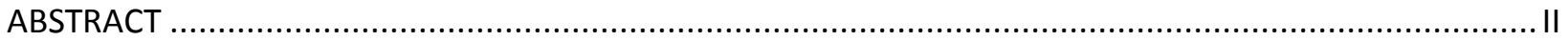

CHAPTER 1: Analysis of Lower Limb Injuries in Frontal Crashes ..................................................... 5

1.1 Current Situation of Lower Extremity Injuries................................................................ 5

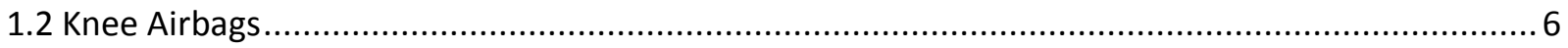

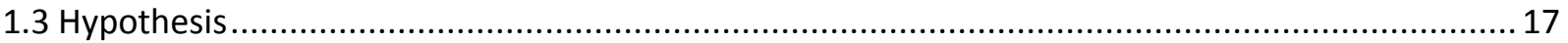

CHAPTER 2: Analysis of Knee Airbag Tests for In-Position Drivers........................................................ 18

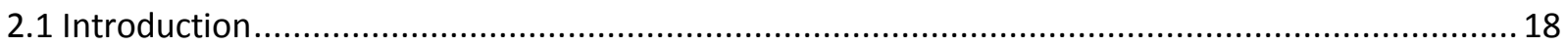

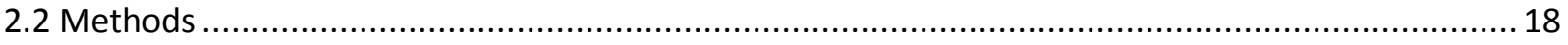

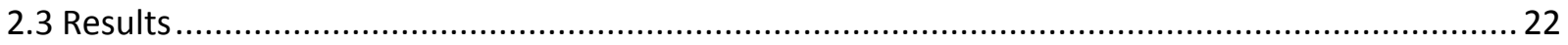

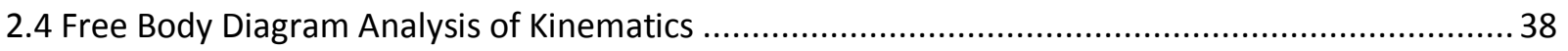

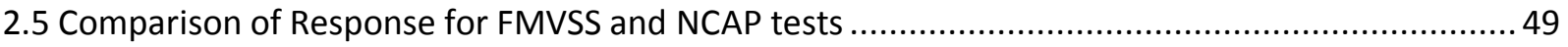

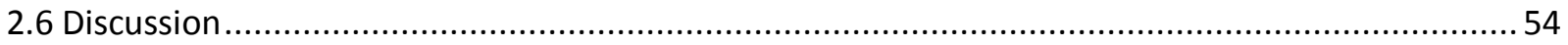

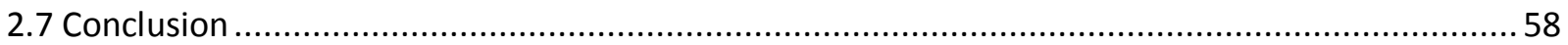

CHAPTER 3: Analysis of Knee Airbag Tests for Out-Of-Position Drivers ............................................. 59

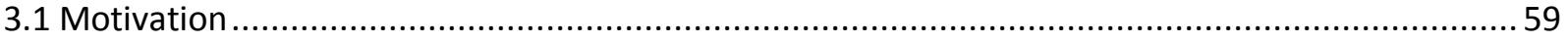

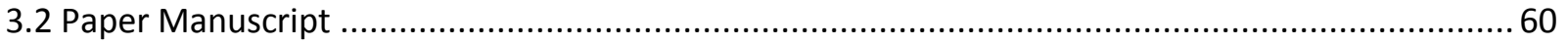

CHAPTER 4: General Discussion, Conclusion and Future Work …..................................................... 76

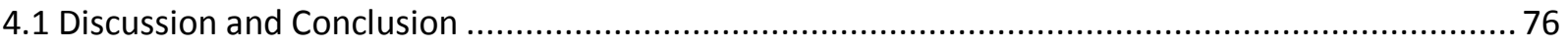

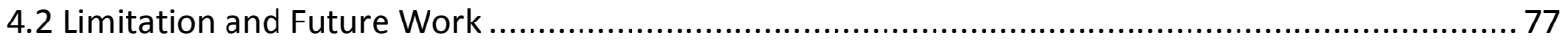

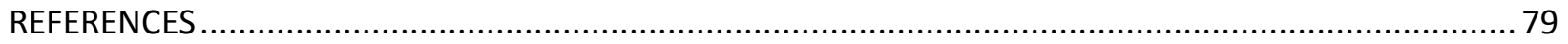

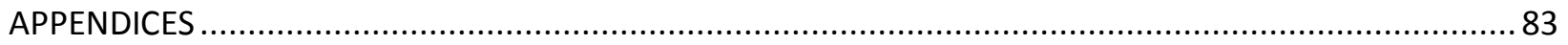

Appendix A. Tests data from FMVSS208 and NCAP tests .......................................................... 83

Appendix B. Summary of dummy positioning for all out-of-position tests ...................................... 97

Appendix C. Airbag mass flow rate and tank pressure during deployment for out-of-position tests ... 98

Appendix D. Injury assessment reference values from IIHS .......................................................... 98

Appendix E. Tests data for out-of-position knee airbag tests ..................................................... 99

Appendix F. Data collection of vehicle fleets equipped with knee airbag in the U.S market ............. 112 


\section{CHAPTER 1: Analysis of Lower Limb Injuries in Frontal Crashes}

\subsection{Current Situation of Lower Extremity Injuries}

Previous studies have emphasized the increasing relative importance of lower extremity injuries in frontal crashes as belt usage and airbag availability has improved the overall performance for occupants (Dischinger et al., 1996; Crandall et al., 1997; Kuppa et al., 2001). A recent study on the data examination of the National Automotive Sampling System - Crashworthiness Data System indicated lower extremity injury still remains the most common AIS 2+ injury sustained in frontal motor crashes (Ye at al., 2015). The distribution of injuries by body regions for drivers who sustained at least one AIS $2+$ injury in frontal crashes revealed that lower extremity injuries were the most frequent injured body region, accounting for $46.3 \%$ of all the injuries (Figure 1). Compared with previous results with a comparable selection criterion, these results indicated an increased proportion of lower extremity injuries as compared to the $28.2 \%$ of lower extremity reported by Kuppa et al. (2001).

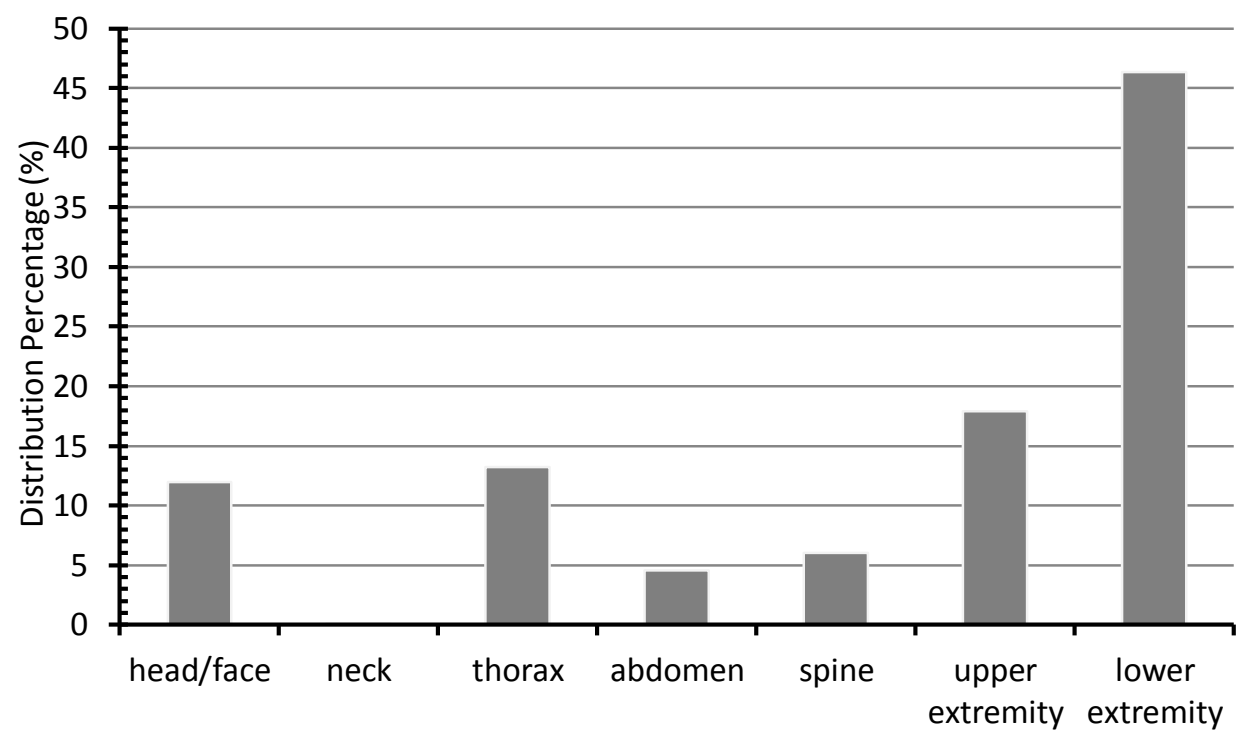

Figure 1. Distribution of injured body regions for drivers with at least one AIS $2+$ injury in frontal crashes

In addition, a breakdown of AIS 2+ injury distribution showed that ankle/foot injuries were the leading lower extremity injury region (Figure 2 ). Ankle/foot AIS $2+$ injuries account for $60.2 \%$ of all driver AIS $2+$ lower extremity injuries. Of all the drivers who sustained at least one lower limb injury, $36.6 \%$ suffered knee injuries as the second highest injured lower limb region. 


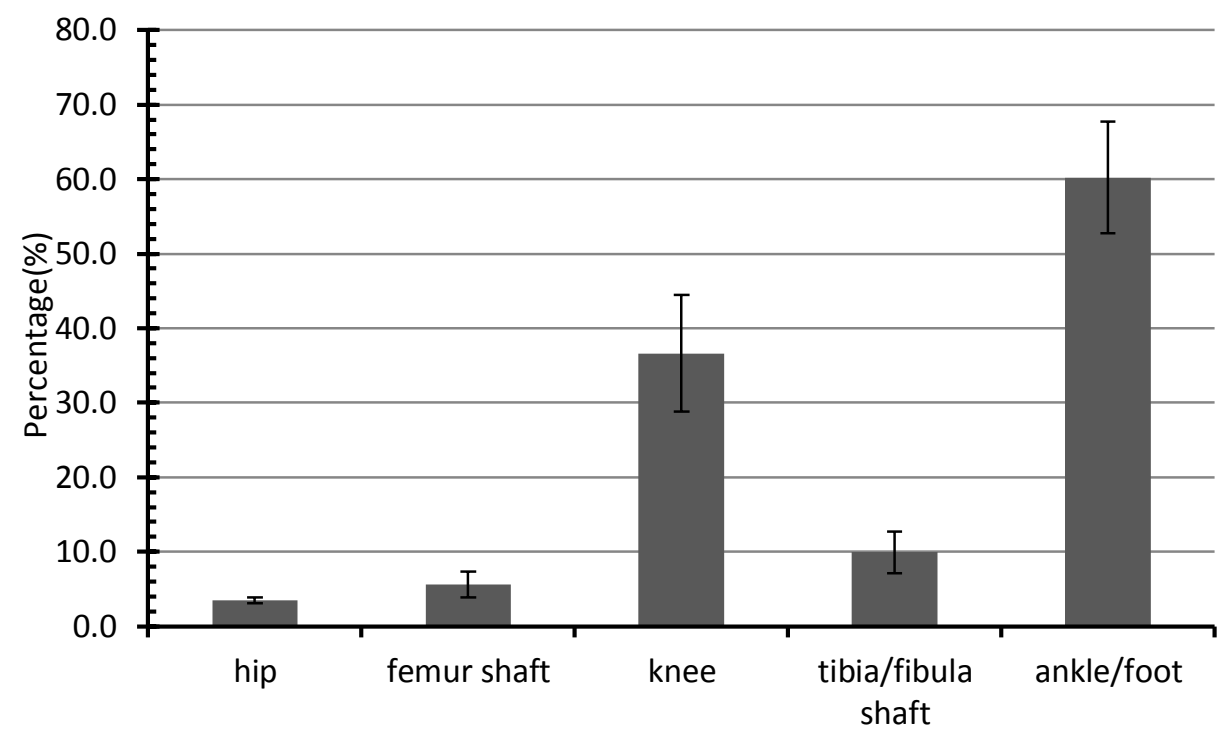

Figure 2. Distribution of injured lower limb regions for drivers sustaining at least one AIS 2+ lower limb injury in frontal crashes

Despite the steady improvement resulted from changes in vehicle structural design and stiffness, it is clear that lower extremity injuries among vehicle occupants remains a prominent issue of concern.

\subsection{Knee Airbags}

\section{Countermeasures for Lower Limb}

Although intrusion of the toe pan was found to be a significant predictor of injury, the reduction of toe pan intrusion is only a partial solution for reducing lower extremity injuries (Ye et al., 2015). With the improvement of vehicle stiffness, many crashes produce little or no intrusion. This is supported by the fact that the majority of drivers (73\%) with lower limb AIS 2+ injuries were involved in crashes with less than $2 \mathrm{~cm}$ of toe pan intrusion. Similarly, $85.3 \%$ of drivers with AIS $2+$ injuries included in this study had little or no intrusion to the instrument panel. The direct causal relationship remains between deceleration of vehicle components and the lower extremity injury outcome. As a result, further consideration should be taken inside the vehicle compartment to address the issue, and multiple countermeasures have been developed to possibly mitigate the lower limb injuries correspondingly.

Inflatable Carpet (InCa) was designed as a device to reduce loadings imparted to the lower limb for varied foot positions, with the design concept of protecting the ankle and foot region of frontal occupants with toe pan intrusion (Rudd et al., 2001; Haland et al., 1998). The InCa inflates to a thickness of approximately $70 \mathrm{~mm}$ at the central chamber and covers an area of about $450 \times 350 \mathrm{~mm}$ (Figure 3). The airbag is usually covered with the vehicle interior carpeting. The plastic tabs inserted through slots in the air bag fabric are used to hold the bag to the load distributor, and belt webbing is used to restrain the entire assembly 
to the toe pan. Results indicated that the InCa reduced foot accelerations, tibia axial loading and ankle dorsiflexion, but the associated efficacy was sensitive to multiple factors including knee bolster design, InCa geometry and driver posture, as well as intrusion onset time.
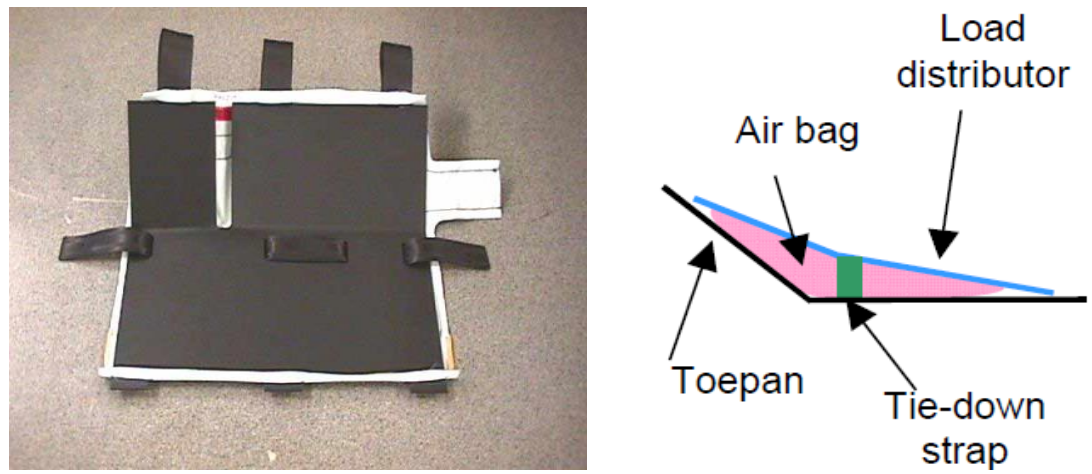

Figure 3. Inflatable Carpet with load distributor and strap (Left); Lateral view of inflatable Carpet (Right)

(Rudd et al., 2001)

The Cushion Restraint Device (CRD) was another countermeasure developed by Breed Technologies, Inc. (currently Key Safety Systems, Inc., Sterling Heights, Michigan) (Figure 4). CRD was designed to reduce pelvic displacement relative to the vehicle by elevate the seat cushion, and to reduce the interaction between the lower extremities of the occupant and the vehicle interior structure. The seat structure may employ an airbag, a mechanical device or a pyrotechnic device, which elevates on the frontal part of the seat cushion to remove the leg and foot from the path of the intruding or reactive structures, which also better controls the upper torso as an effort to mitigate lower limb injuries accordingly.
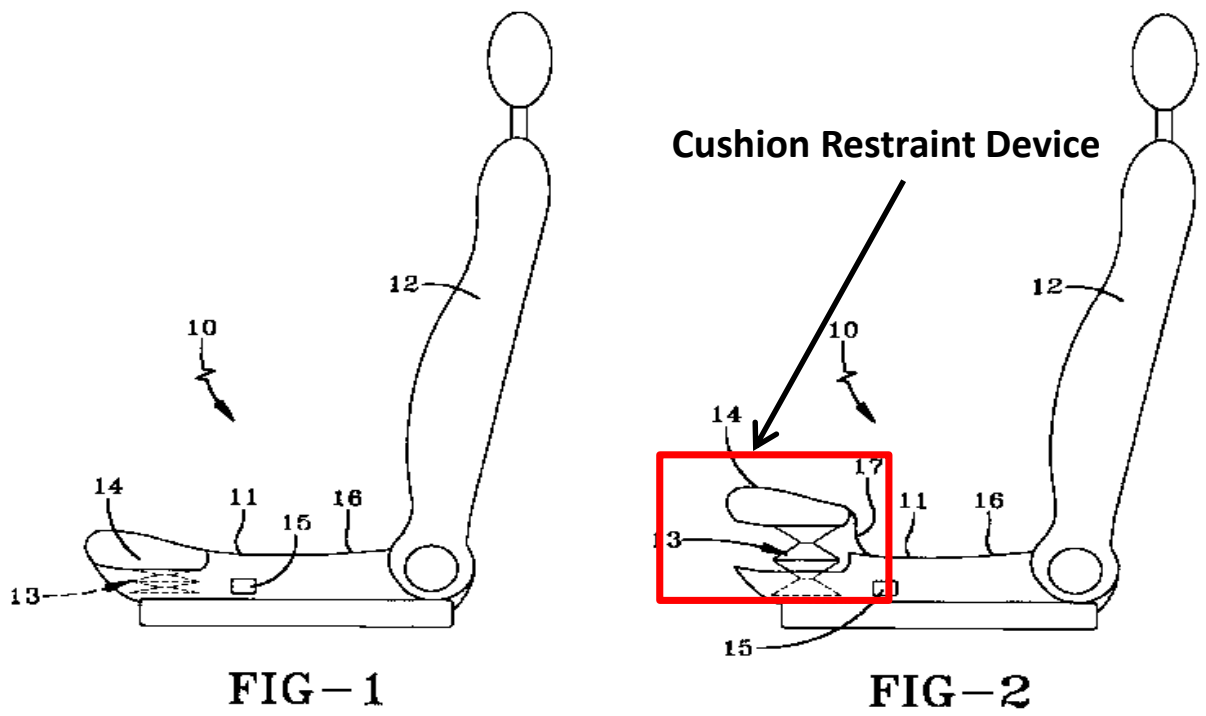

Figure 4. Description of the seat cushion restraint system (US Patent: US 5695242 A, 1997) 
Another advanced restraint system was the Pelvis Restraint Cushion (PRC) (Faure et al., 2007). Similar with the CRD, the PRC consisted of an inflatable metal box located beneath the occupant femur, and the deployment of such device reduced the pelvis forward movement, which optimized the occupant posture when coupled with the buckle or retractor pre-tensioner (Figure 5). Double pretension system was claimed to significantly reduce the knee contact with instrument panel, and reduce the lower extremity injuries accordingly.

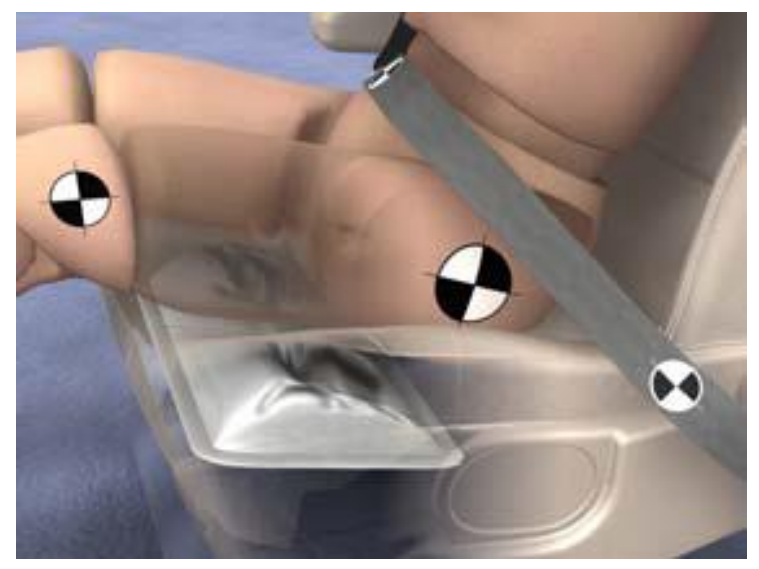

Figure 5. Pelvis Restraint Cushion deployed after interaction with occupant (Faure et al., 2007)

All these deployable devices were designed to provide protection and restraint prior or at early stage of a crash, as a method to reduce the interaction between the lower extremities with the vehicle structures inside the compartment and to absorb the impact energy, which better control the occupant kinematics and prevent the lower limb injuries.

\section{Knee Airbag Prevalence}

Of all the inflatable countermeasures considered, knee airbag was touted as one of the most representative countermeasures inside vehicle compartment in current market. Restraint of the lower limb from the knee bolster was an important factor to consider throughout the development of restraint systems (Culver et al., 1979). Although structural components, style and design may vary, knee bolsters are generally designed to distribute lower extremity contact loading and to absorb overall occupant energy. Knee airbags are located underneath the instrument panel above the foot-well area, and are designed to deploy simultaneously with the frontal airbag (Masuda, 2014). When a collision occurs, knee airbags inflate to fill in the space between the dashboard and the occupant's lower extremity. The world's first knee airbag was used in the 1996 model Kia Sportage vehicle. Since then, the number of vehicles equipped with knee airbags has increased gradually (Appendix C). With the increasing prevalence in market penetration, knee airbag is anticipated to be assembled in $46 \%$ of the vehicle fleets as a passive restraint system in North America by the year 2018 (Figure 6). 


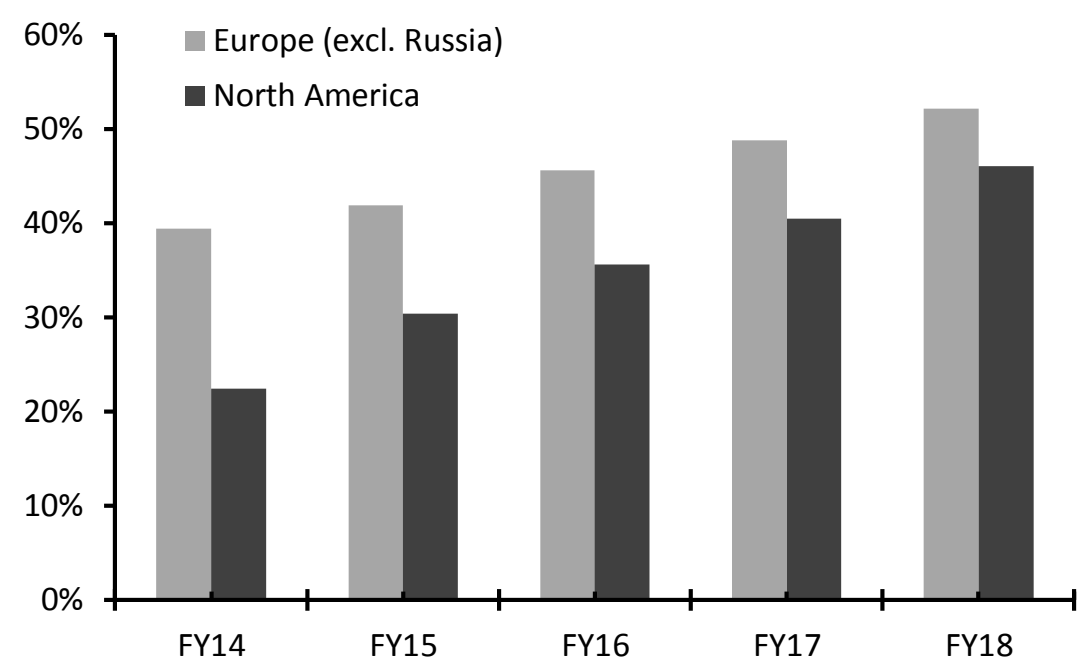

Figure 6. Prevalence of knee airbag from market data forecast (source: Takata Corporation, 2014)

\section{Knee Airbag Targets}

The targets of an active knee airbag concept include multiple benefits for the overall occupant performance (Figure 7).

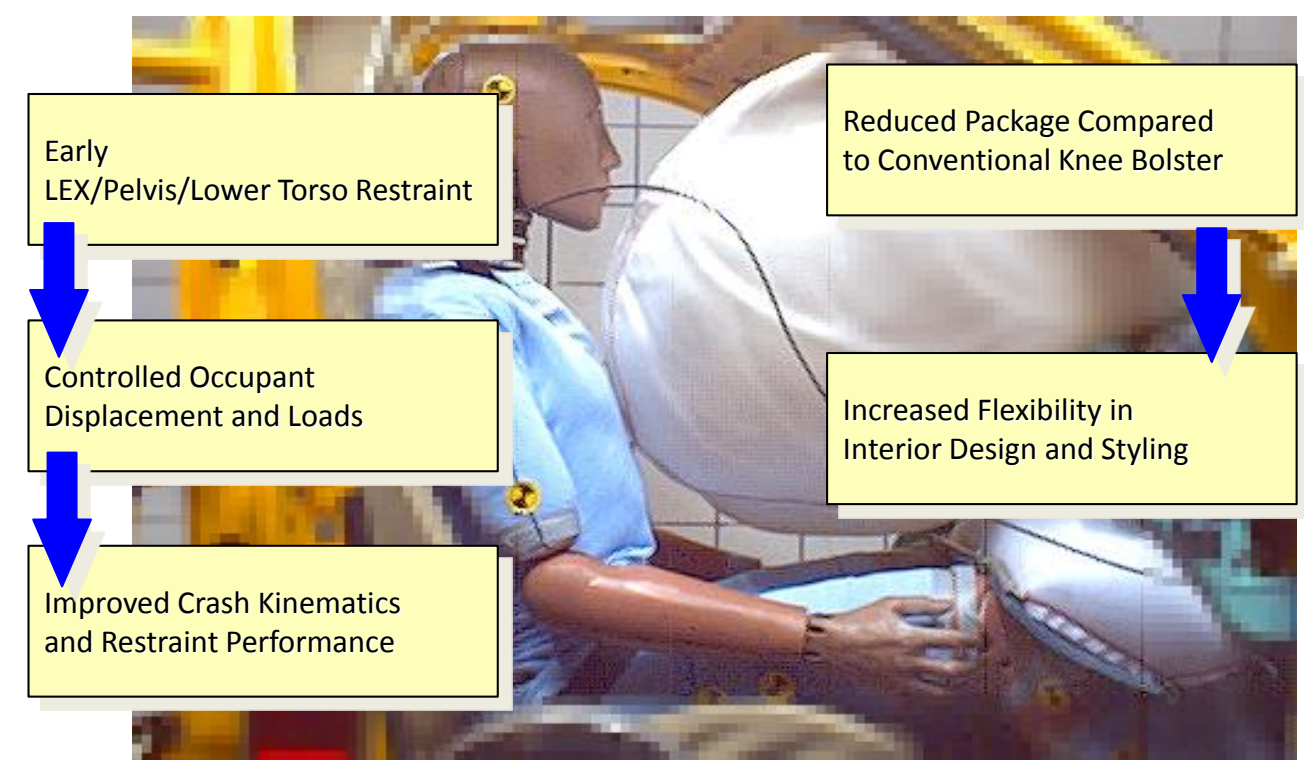

Figure 7. Target of an active knee airbag system (source: website of TRW Automotive Inc.)

Knee airbags are generally designed to reduce pelvis forward motion for the improvement of chest compression and reduction of associated injury risk. For belted occupants, through early torso restraint and controlled occupant displacement by early pelvis restraint, lap and shoulder inner belt loads were also reduced, which resulted the decrease in abdomen and chest loading (Figure 8). For unbelted occupants, the early constraint from the knee airbag deployment could also reduce the chest deflection resulting from the contact with steering wheel, and changes the trajectory of the head against the window shield, which reduced 
the head forward acceleration accordingly. The early restraint provided by knee airbags generally increases the efficiency of occupant kinetic energy absorption (Kato et al., 2007).
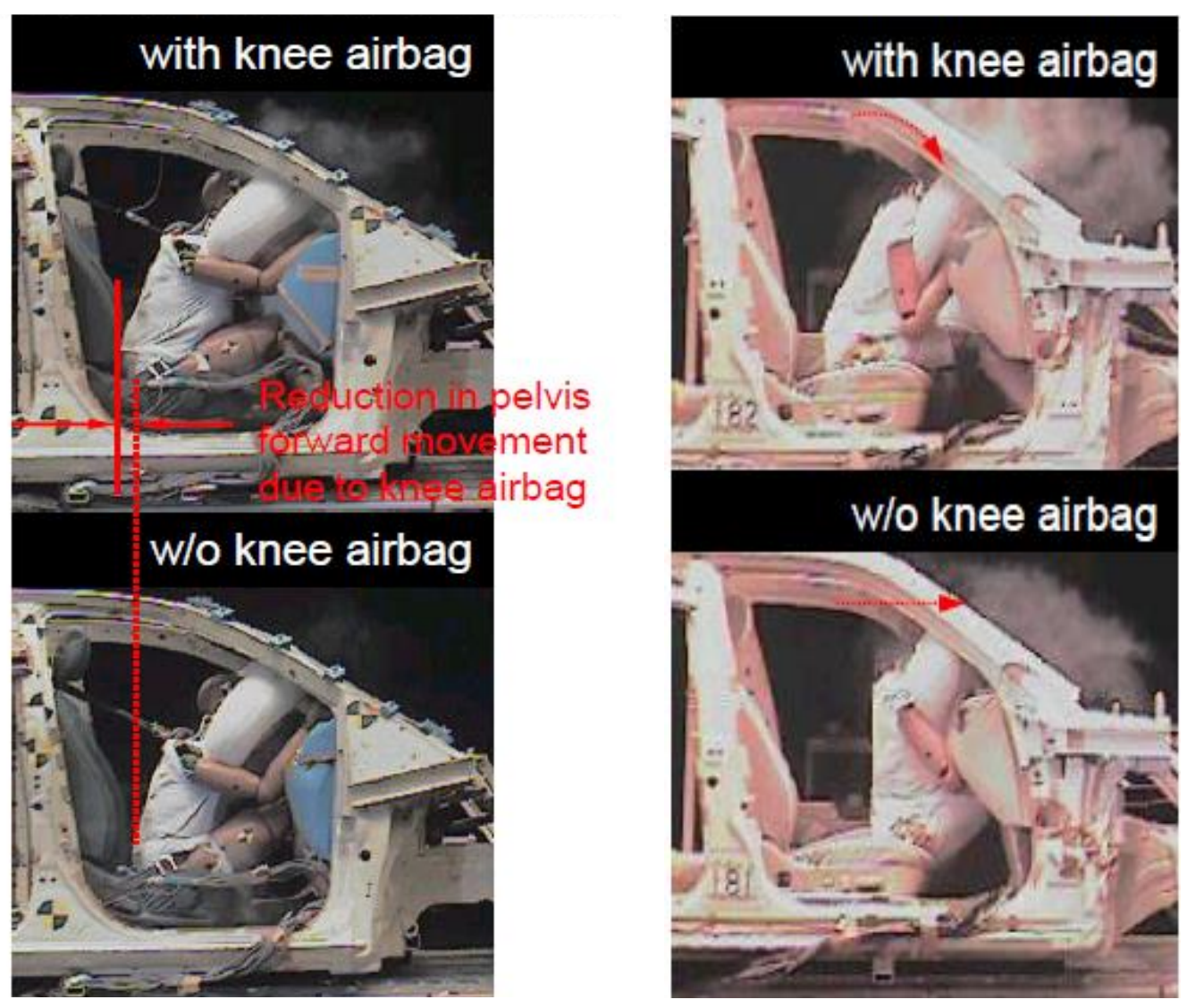

Figure 8. Advantage of knee airbag for belted occupants (Left) and unbelted occupants (Right) (Masuda, 2014)

Knee airbags also aim to mitigate the high femur loadings during the contact between the knee and knee bolster. Load-distributed panel was instrumented for the inflatable knee bolsters, which provided a large contact area with the legs and avoided stress concentration from deployment loads, where fabric knee airbags provided an energy-absorbing cushion to reduce the contact stiffness. In addition, knee airbags could also potentially prevent entrapment of the knees via the removal of vertical constraint, compared to conventional knee bolster (Masuda, 2014). During the deployment, knee airbags tend to wrap around the knees thereby protecting them from interaction with rigid parts inside the instrument panel, including key cylinders and steering column.

Lastly, knee airbags could also reduce the package behind the instrument panel, which provide flexibility in interior design and larger comfort space between the knee and the instrument panel for the occupants (Roychoudhury et al., 2004). This change in design allows for more functional use of the space in the vehicle's interior and a more lightweight solution than metal bars and other traditional knee protection alternatives. 


\section{Knee Airbag Design}

Design parameters of a typical knee airbag include number and size of vent holes, inflated depth at tether, length of deployed cushion, tether length and cushion folding patterns. These parameters may vary given the multitude of knee airbags, and may be updated correspondingly to coordinate with vehicle interior structure and compartment space. Mounting position, for instance, could change correspondingly to fit in with the instrument panel geometry and housing shape. Some vehicles fleets adapt high-mount knee airbags (e.g., 2007 BMW 7 series) while other OEMs apply low-mount types (e.g., 2005 Lexus IS) (Figure 9).
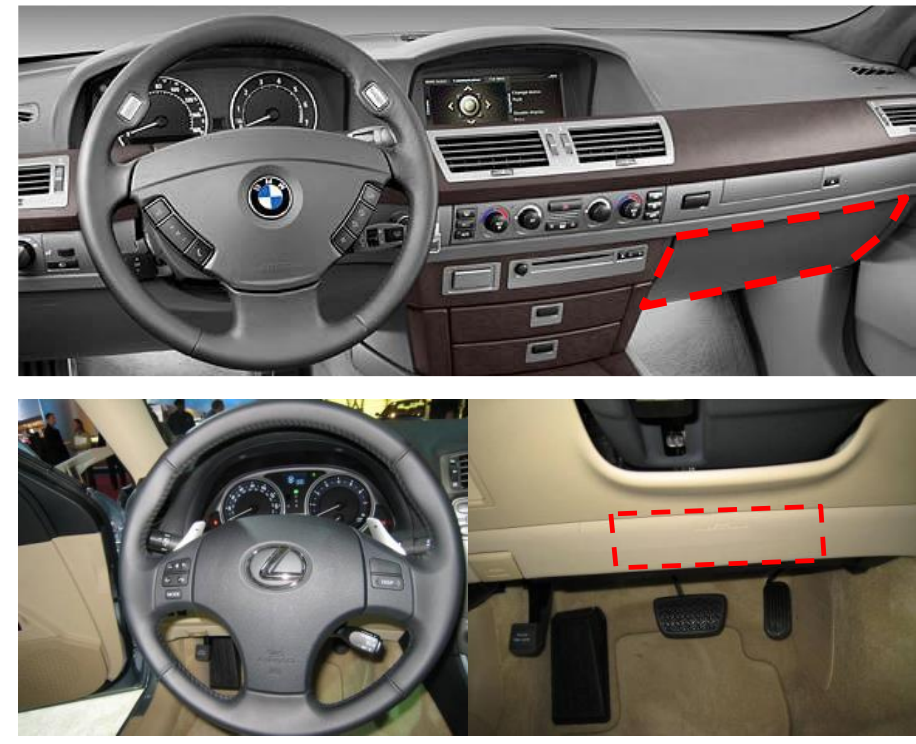

Figure 9. KAB mounting position difference: high-mount in 2004 BMW 7 series (Top);

Low-mount in 2005 Lexus IS (Bottom)

The material of the airbag deployable surface could also vary from plastic (e.g., 2004 BMW 7 series) to steel stamping (e.g., 2004 Grand Caravan). Venting holes greatly affect the deployment pressure and the ensuing occupant kinematics.

Although it might be difficult to account for all types of knee airbags in the field, prevalent types could be generically categorized into fabric knee airbag and inflatable knee bolster (IKB), respectively. Several differences exist between fabric knee airbag (KAB) and inflatable knee bolster (IKB). The fabric knee airbag referred to the airbag cushion been folded and packaged below the passive knee bolster system, while inflatable knee bolster is usually located in the door of the glove box, and inflates quickly with inserted inflator pump and a load displacing panel. IKB deploys the knee airbag cushion inside the instrument panel surface and indirectly restrain the occupant lower extremity from the deploying panel, while the fabric KAB restrains the occupants' knees from the direct deployed airbag cushion. Compared to fabric knee airbags, IKB provided 
a more simple and cost-effective alternative in terms of deployment shape and airbag folding pattern, but the efficiency between these two types of knee airbags remains to be investigated (Roychoudhury et al., 2004). Additionally, the inflator pressure from the two types of knee airbags could vary, which lead to the change of deployment aggressiveness and ensuing lower extremity response.

Recent vehicle models of Audi A8, Toyota Camry are generally equipped with the fabric knee airbag, and IKB could be found in vehicle makes including BMW 745i, Mercedes Benz and Chrysler Pacifica (Figure 10) (Hong et al., 2007; Roychoudhury et al., 2004).
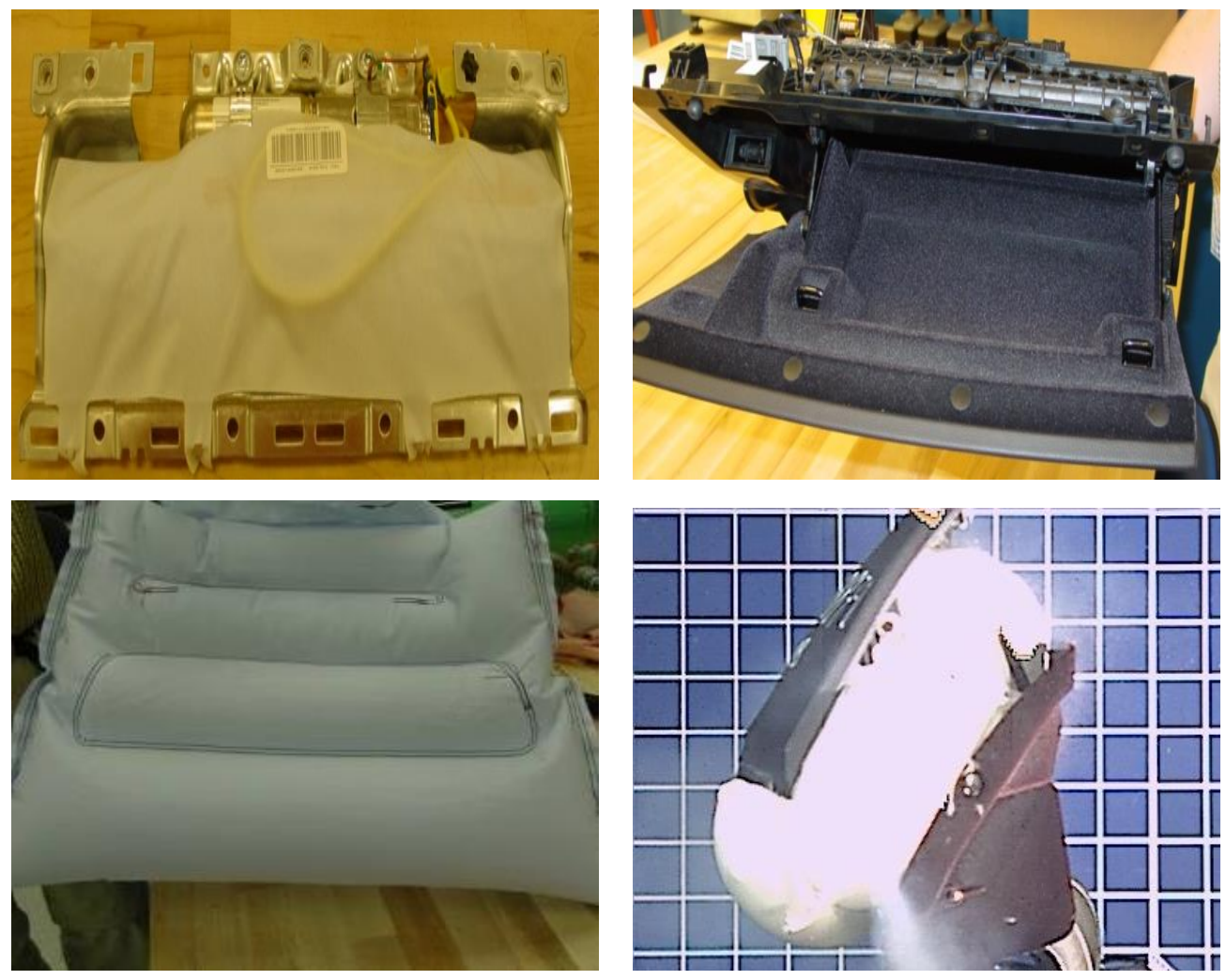

Figure 10. Fabric Knee Airbag in 2004 Audi A8 (Left); Inflatable Knee Bolster in 2004 BMW 7 series (Right) 


\section{Knee Airbag Effects}

Several studies have previously examined the lower extremity response under knee airbag deployment. Malczyk et al. investigated the knee airbag effects on loading of the lower extremity (Malczyk et al., 2003). Static out-of-position tests were conducted using the Hybrid III $5^{\text {th }}$ percentile dummy to explore the load effects of KAB in different module positions, with dummy knees placed against the lower instrument panel. A production knee airbag was used, with a hybrid inflator (160 kPa tank pressure, 28.3 Liter tank) and a 14 Liter silicone-coated cushion without vent holes. Seating height was varied to represent three different vertical alignments of the lower limb relative to the KAB module, and the instrument panel was reinforced from the original OEM design (Figure 11).
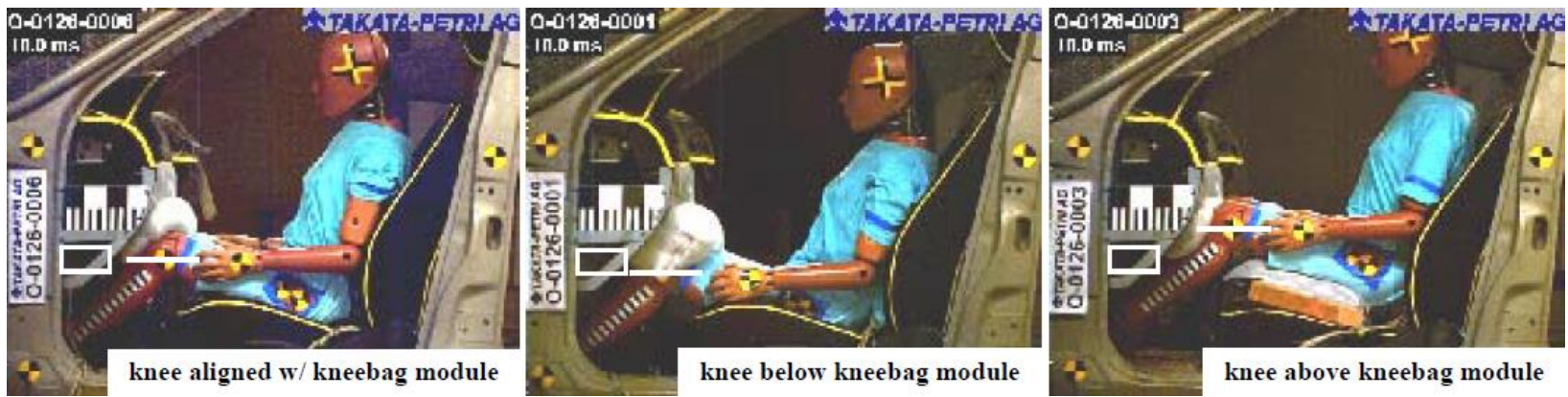

Figure 11. Out-of-position configurations (Malczyk et al., 2003)

Results indicated clear differences in peak loading from the vertical alignment change of the KAB module, with the highest loading found in case where the knee positioned above the $K A B$, which is the same location of the unfolding cushion when it was inflated. Kinematics of the lower limb also varied from in-position to outof-positon tests where abduction of the legs was observed during the deployment of KAB. Although this study provided valuable information of knee airbag effects on out-of-position drivers, and revealed the higher injury risk of lower extremity for small adults, the small sample size $(n=3)$ was obviously insufficient to derive any conclusive findings on knee airbag performance. Additionally, changes in driver out-of-position posture, including knee to instrument panel distance, knee-to-knee distance, could potentially change the lower extremity response accordingly.

Leport et al. performed three out-of-position KAB static deployment tests with the Hybrid III $50^{\text {th }}$ percentile dummy and two tests with the $50^{\text {th }}$ percentile PMHS(Leport et al., 2009). All tests were performed using a rigid test fixture with the geometry typical of a mid-size vehicle. The dummy was seated with the knees moved together, the feet on a rigid foot rest and the heels in contact with a fixed horizontal plane. The seat was then translated forward till the knees contacted the rigid profile of steering column. Three dummy tests and two PMHS tests were performed in the same posture, with the only change of tibia to airbag module distance (55 to $67 \mathrm{~mm}$ for Hybrid III dummy tests, $53 \mathrm{~mm}$ and $54 \mathrm{~mm}$ for two PMHS tests, respectively) (Figure 12). 


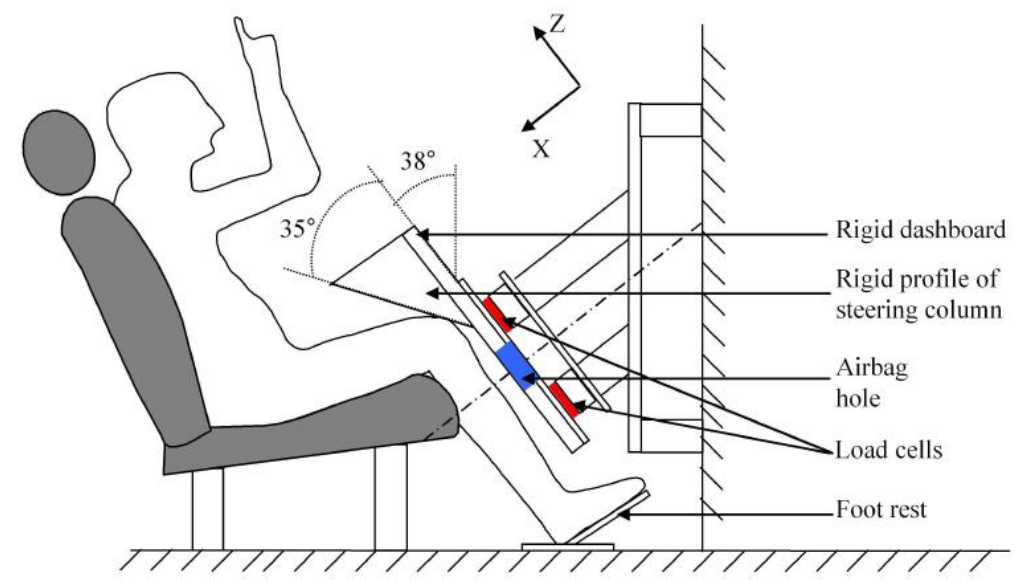

Figure 12. Test setup for Out-of-position knee airbag tests (Leport et al., 2009)

Abrasions and bruises were observed in the cadaver tests from this study, but no bone or ligament injury was found. Low risk of lower limb injury was also found associated with the dummy tests but the study by Leport et al. was limited by sample size and dependence of KAB design.

Another cadaveric study was performed with three KAB (18 Liter volume) static deployment tests, and with the PMHS positioned in normal seating position, knee flexed above 90 degrees and knee flexed below 60 degrees, respectively. An additional dynamic test $(48.8 \mathrm{~km} / \mathrm{h})$ with a belted PMHS was also performed in the normal seating position (Figure 13).

Static Test 01

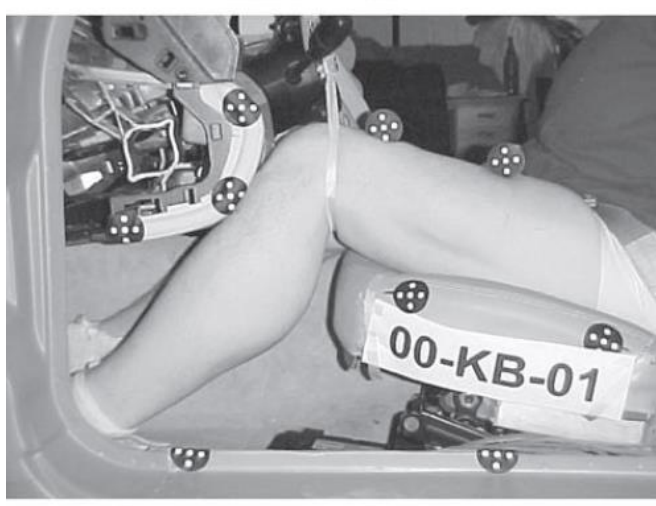

Male, 45 years, $167 \mathrm{~cm}, 83 \mathrm{~kg}$

Direct contact between lower leg/ knee joint and knee bag cover. Lower leg in drive position
Static Test 02

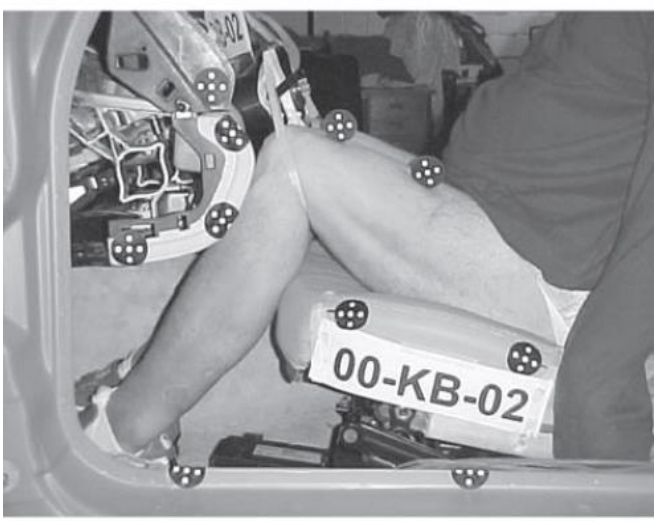

Male, 67 years, $161 \mathrm{~cm}, 85 \mathrm{~kg}$

Lower leg nearly vertically.

Load directly on lower leg underneath knee 


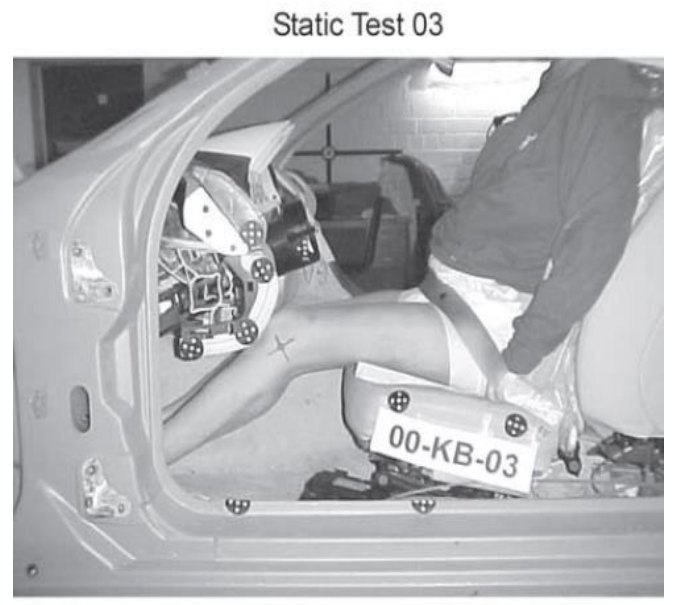

Female, 42 years, $161 \mathrm{~cm}, 62 \mathrm{~kg}$

Flat angle between thigh and lower leg. Load on knee joint area
Dynamic Test 04

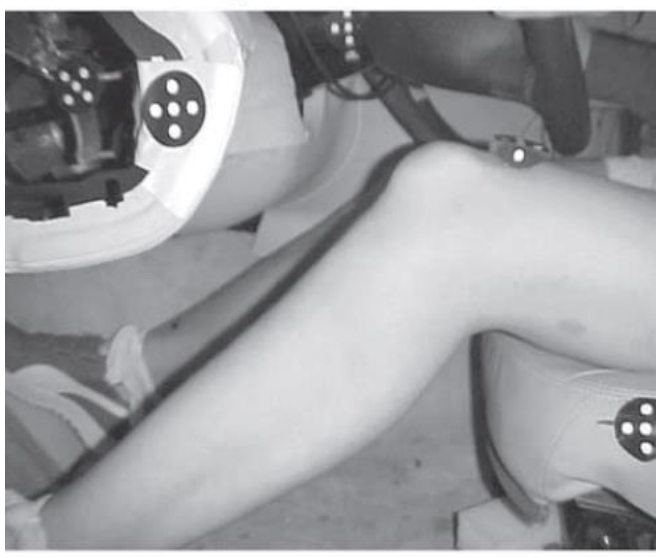

Female, 36 years, $171 \mathrm{~cm}, 64 \mathrm{~kg}$

Flat angle between thigh and lower leg. Load on knee joint area

Figure 13. Description of three static deployment tests and one dynamic test with KAB

(Schroeder et al., 2005)

Apart from some skin lesions in the impact area, no bony fracture and no knee ligament or tendon injuries were observed from all the tests. Abduction of both legs was noticed in some of the tests, but no increased injury risk for the lower extremity could be derived from the study (Schroeder et al., 2005).

Two recent publications have analyzed data from the Crash Injury Research and Engineering Network (CIREN) and National Automotive Sampling System Crashworthiness Data System (NASS-CDS) to evaluate realworld lower extremity injury risk in frontal crashes. Weaver et al. compared 9 CIREN cases with knee airbag to 183 no-knee airbag cases of the same vehicle model, crash type, and severity using a similarity scoring algorithm (Weaver et al., 2013). Results indicated a statistically significant reduction in femur fractures, but an increased incidence rate of proximal tibia/fibula and foot/ankle fractures (also statistically significant), for occupants in crashes with deployed knee airbags. While the study by Patel et al. combined NASS-CDS and CIREN data to maximize case availability for a matched cohort study, there were still an insufficient number of cases to find statistical significance of the lower extremity injury risks, although a decreased risk of hip and thigh fracture, and an increased risk of tibia/fibula and foot fracture were identified (Patel et al., 2013).

Another study using crash, occupant, and medical information, was performed on the selected cases from the CIREN database by University of Virginia, Center for Applied Biomechanics (Crandall et al., 2014). Eligibility and criteria for case inclusion consisted of drivers older than 16 years of age that were involved in a single event, frontal plane crash. Frontal collision was determined by occurrence of most severe vehicle crash damage (i.e., Rank 1) in the frontal plane with principal direction of force (PDOF) ranging between positive and negative 20 degrees. All cases must have the presence and deployment of the knee airbag, and sustained at 
least one lower extremity injuries, including bony fractures, skin contusions and abrasions. The query yielded a final inclusion of 29 cases with at least one lower limb injury, with leg abrasion, tibia shaft fracture and fibula shaft fracture as the leading injuries (Figure 14). As current injury criteria of the lower extremity lack the capability of predicting the injury location and injury pattern, this study specifically revealed an increased injury risk of the tibia shaft and fibula shaft region. While these injuries occur with the deployment of the knee airbag in moderate Delta- $\mathrm{V}(49.5 \pm 17.5 \mathrm{~km} / \mathrm{h})$, the injury source remains indeterminate, and the influence of lower extremity response from the knee airbag deployment requires further investigation.

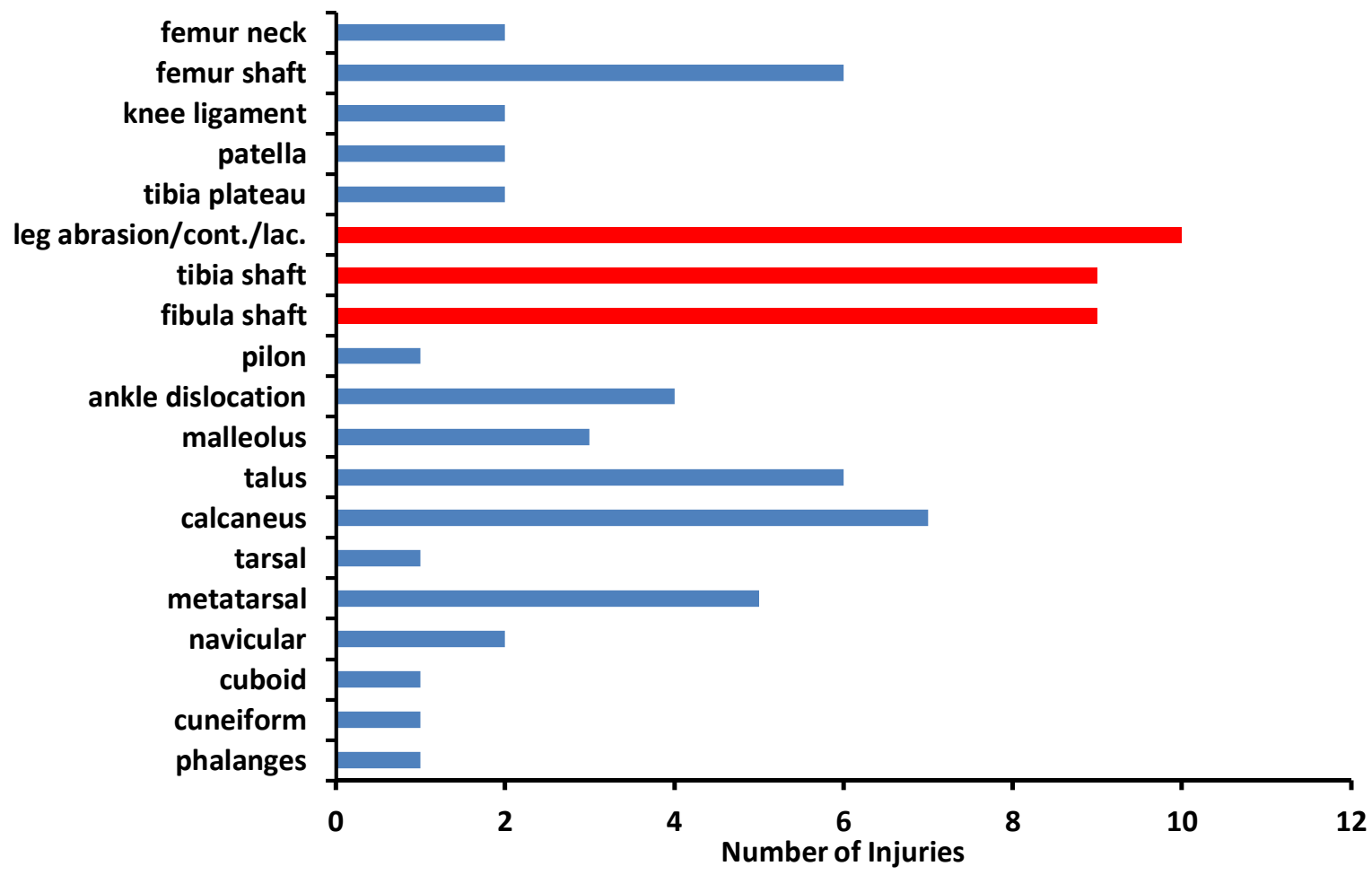

Figure 14. Injury summary of queried CIREN cases (Crandall et al., 2014)

Beyond these studies, relatively little has been published regarding the performance of knee airbags in real-world crashes, despite their increasing market penetration. Part of the difficulty results from the fact that there are a multitude of different airbag configurations as previously mentioned (bottom-deployed or reardeployed, high-mount or low-mount, KAB size, inflator output, etc.) that confound the assessment of KAB performance in retrospective field studies. Given the lack of understanding of real-world performance, questions arise regarding the knee airbag effectiveness in different frontal crash scenarios, the limitations of airbag coverage and overloading, the changes in injury patterns relative to knee bolsters, and the potential for lower limb injuries resulting from deployment of the knee airbag itself. 


\subsection{Hypothesis}

From the review of previous experimental studies and findings of lower limb injuries under knee airbag deployment in real-world cases, the research question of this thesis was whether knee airbags could sufficiently protect the lower extremity in different frontal crash scenarios, or could potentially increase the lower limb injury risk as a tradeoff for overall occupant benefits. This thesis investigated the deployment of both inflatable knee bolster and cushion knee airbags, as both types of knee airbags were introduced as additional passive safety restraint systems to improve the vehicle performance for a wide range of test conditions, as OEMs are now required to evaluate the $5^{\text {th }}$ percentile female and the $50^{\text {th }}$ percentile male in both belted and unbelted driving scenarios with the requirement of FMVSS 208.

The first part of the thesis aimed to investigate the lower extremity response for drivers in nominal driving posture, under dynamic condition with the presence of an Inflatable Knee Bolster (IKB). The IKB selected was a production airbag product and all the tests were performed in a buck derived from a mid-size sedan profile, which aims to reproduce the real-world scenarios with KAB in both belted and unbelted conditions. A total of seven unbelted FMVSS208 tests and eight belted NCAP tests were performed with a $50^{\text {th }}$ percentile Hybrid-III dummy to investigate the IKB effects on the lower limb. The study examined the loading path difference with and without the knee airbag in the dynamic condition, and assessed the injury risk of tibia/fibula and foot/ankle region associated with an IKB deployment.

The second study aimed to investigate the biomechanical response of the lower extremities during knee airbag deployment in various out-of-position driving scenarios. The 5th percentile female was chosen for an investigation of knee loading resulting from interaction with a deploying knee airbag to represent the most vulnerable group for sustaining lower limb injuries, given their diminished lower injury tolerance and a closer seating proximity to the instrument panel/ knee bolster (Mertz, 1993; Reed et al., 2014). A total of 11 static knee airbag deployment tests were performed with a 5th percentile female Hybrid-III dummy outfitted with either the original Hybrid-III lower extremities or the 5th percentile THOR-FLx. This study used dummy tests to assess the potential for out-of-position lower extremity injuries upon knee airbag deployment and to observe how knee airbag deployments could alter the occupant's positioning during a crash.

The following null hypothesis was proposed and the thesis aimed to evaluate the proposed hypothesis:

$H_{0}$ : Between standard knee bolsters and knee airbags, there is no difference in injury risk of the tibia/fibula and foot/ankle regions for drivers in frontal motor vehicle crashes. 
Correspondingly, the alternative hypothesis was derived as:

$H_{1}$ : Between standard knee bolsters and knee airbags, differences exist in injury risk of the tibia/fibula and foot/ankle regions for drivers in frontal motor vehicle crashes.

\section{CHAPTER 2: Analysis of Knee Airbag Tests for In-Position Drivers}

\subsection{Introduction}

Previous dynamic evaluations with knee airbags have shown benefits to improve overall occupant performance, compared to standard knee bolsters (Jenkins et al., 2002; Roychoudhury et al., 2004; Sohr et al., 2009). Recent US-NCAP tests indicated that the application of a knee airbag reduces the overall injury risk by $25 \%$ in the test speed of $56 \mathrm{~km} / \mathrm{h}$, which is a composite percentage derived from the overall injury risk of head, check, neck and femur regions (Sohr et al., 2009). However, real-world data suggested an increased risk of tibia/fibula and foot/ankle regions under knee airbag deployment (Weaver et al., 2013; Patel et al., 2013). For current study, measurement data for the lower extremity was fully examined to evaluate the proposed hypothesis of the thesis, and more specifically, it is assumed that knee airbags have adverse effects to increase the injury risk of tibia/fibula and foot/ankle regions for drivers in frontal crashes.

\subsection{Methods}

Both FMVSS208 tests and NCAP tests were performed with the $50^{\text {th }}$ percentile Hybrid III Anthropometric Testing Device. Dummy positioning for unbelted FMVSS208 tests and belted NCAP tests both followed the standardized dummy positioning procedure, with measurement matched up with the reference values (Table 1).

Table 1. Summary of ATD dimensions reference for positioning

\begin{tabular}{|c|c|c|c|}
\hline \multicolumn{4}{|c|}{ Standard Dummy Positioning for FMVSS208 and NCAP tests } \\
\hline ATD Dimensions & Reference & ATD Dimensions & Reference \\
\hline Nose to Upper Rim & $387 \mathrm{~mm}$ & $\begin{array}{c}\text { Left knee to bolster } \\
\text { (closet point) }\end{array}$ & $90 \mathrm{~mm}$ \\
\hline Nose to I.P. (closest point) & $527 \mathrm{~mm}$ & $\begin{array}{c}\text { Right knee to bolster } \\
\text { (closet point) }\end{array}$ & $105 \mathrm{~mm}$ \\
\hline Nose to center of module & $380 \mathrm{~mm}$ & Hip "Y" to Rocker & $185 \mathrm{~mm}$ \\
\hline Chest to center of module & $280 \mathrm{~mm}$ & Knee "Y" to Rocker & $210 \mathrm{~mm}$ \\
\hline Knee spacing (center to center) & $235 \mathrm{~mm}$ & Lower abdomen to rim & $140 \mathrm{~mm}$ \\
\hline Pelvic angle (FMVSS 208 tests) & $21.5^{\circ}$ & Pelvic angle (NCAP tests) & $22^{\circ}$ \\
\hline
\end{tabular}


Driver frontal airbag was mounted with General Motors X-130 driver airbag module hardware, and airbag covers were molded with molded tear seams and manually scored edges (Advanced Driver Pyrotechnics System version 1(ADPS-1) inflators, 178/229 kPa and 125/174 kPa). The airbag cushion of the inflatable knee bolster used in the tests was placed behind an injection molded plastic cover, which is the Class A surface of the knee bolster. The inflator represented one of the most advanced dual-stage inflator products developed by AutoLiv, Inc. at the time when tests were performed, which used pyrotechnics that provide low temperature gas to the airbag upon deployment. The IKB utilized an AutoLiv Slide Hybrid (ASH-2) inflator(182 kPa), which was an innovation developed by AutoLiv Inc. that used the chemical process of dissociation rather than combustion to produce a heated gas during the airbag inflation, which avoid the high temperature to potentially burn the occupants compared to other inflator designs including compressed stored gas, pyrotechnic, and hybrid. The advantage of ASH-2 inflator was the elimination of separate fuel and oxidant charges, and improvement of reliability with lighter weight and smaller package size. Additionally, this type of inflator allows the airbags to remain inflated for a longer time. Therefore, the tests were deemed representative of the general IKB applicability.

For belted NCAP tests, production 2001AN seatbelt was used with seatbelt height adjuster placed at midposition; along with $4.0 \mathrm{kN}$ load limiting retractor and no pre-tensioner. Seat was placed at 24 degrees seatback angle and middle track position for all FMVSS208 and NCAP tests. Finalized dummy positioning followed the SAE procedure and matched with the FMVSS208 and NCAP tests correspondingly (Figure 15).

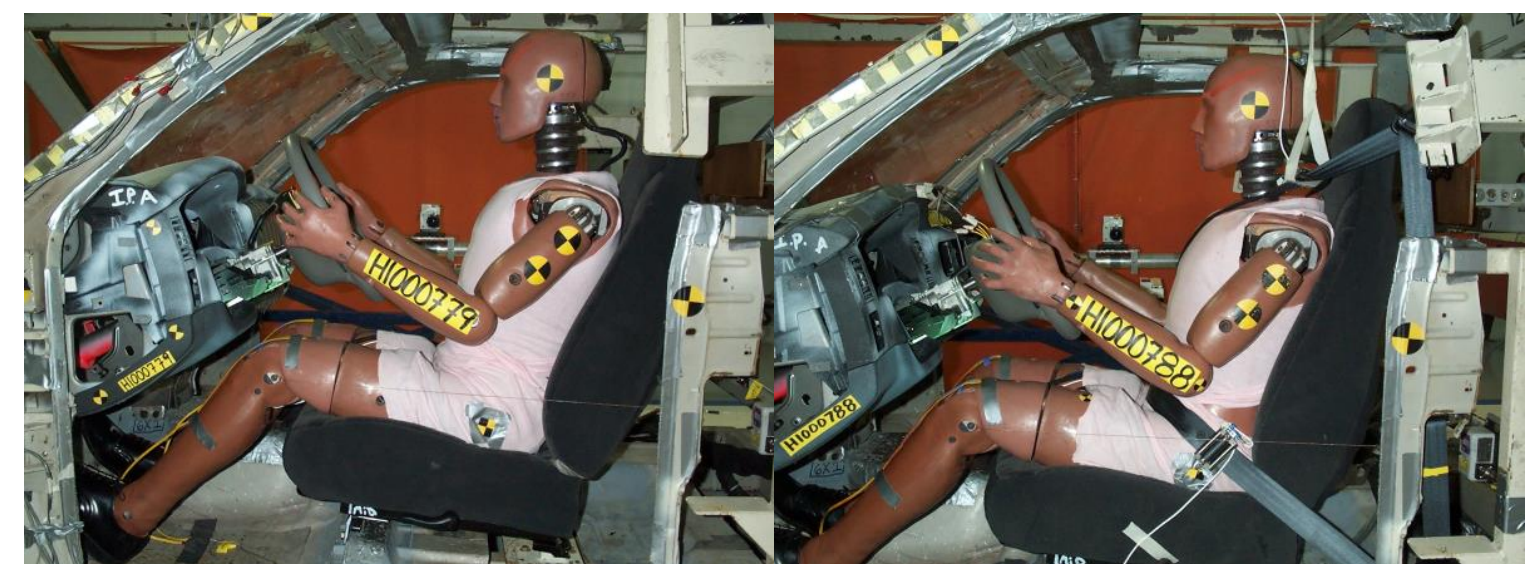

Figure 15. Positioning of FMVSS208 $50^{\text {th }} \%$ unbelted ATD (Left) and US-NCAP $50^{\text {th }} \%$ belted ATD (Right)

The instrumentation of the $50^{\text {th }}$ percentile Hybrid III leg included 1-axis inner and outer knee clevis load cell (FZ), 1 axis femur load cell (FZ), 3-axis proximal and distal tibia load cell (FZ, MX, MY), and knee slider (Figure 16). Toe accelerometer and heel accelerometer were present but the data was not recorded in the tests. 


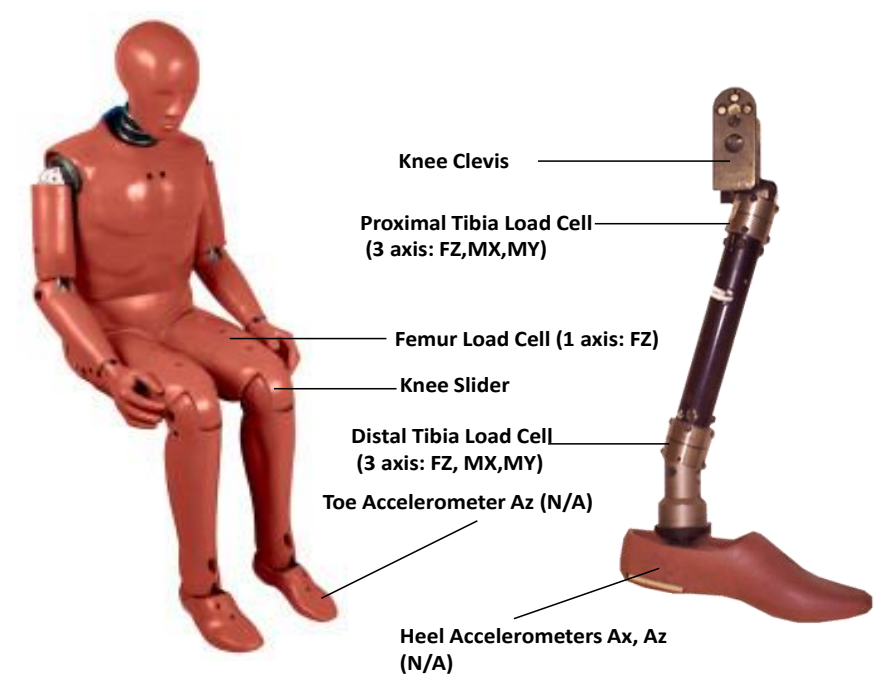

Figure 16. Instrumentation of the Hybrid-III Denton leg

The test matrix consisted of seven FMVSS208 tests, and eight NCAP tests. Inflatable knee bolster, as one version of generic knee airbag, was present in three out of seven unbelted FMVSS208 tests, and four out of eight belted NCAP tests, respectively (Table 2). All FMVSS208 tests were performed at the speed of $40 \mathrm{~km} / \mathrm{h}$, and NCAP tests were conducted at the speed of $56 \mathrm{~km} / \mathrm{h}$. Driver airbag module was set to fire at $18 \mathrm{~ms}$ on first stage fire time after the sled pulse onset time, and $28 \mathrm{~ms}$ at second stage. For tests with inflatable knee bolster, IKB firing time was set at $18 \mathrm{~ms}$, simultaneously with the firing of driver frontal airbag. Test 862 and 863 (depowered frontal airbag, inflatable knee bolster) were completed with driver frontal airbag set not to fire at 2 nd stage, as an approach to further depower the driver airbag inflator, and the tank performance (60 Liter) was $125 \mathrm{kPa}$ for the 1st stage only deployment for these two tests. A preliminary analysis examined the effect of driver frontal airbag inflator pressure upon lower extremity response, and the difference was deemed negligible. Figure 17 demonstrated two examples of tests with different driver airbag inflator pressure. As the figures denoted, starting from onset time to the time when peak loadings of femur compression force and tibia axial force occur, DAB pressure had little effect on the lower extremity response in terms of signal phase and shape, while the magnitude change was mostly resulted from inter-test variability. 

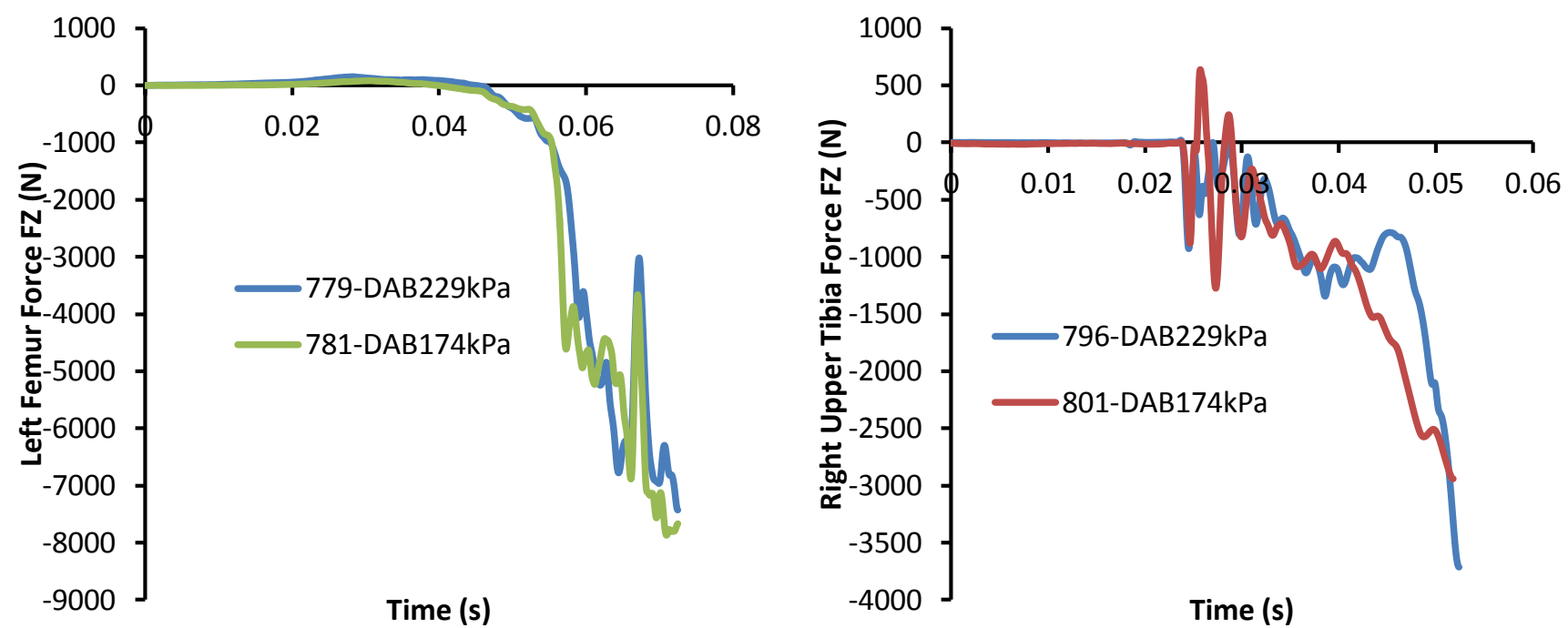

Figure 17. Examples of dummy lower extremity response with different DAB inflator pressure

All the FMVSS208 tests and NCAP tests were grouped to increase the amount of usable data for the evaluation of knee airbag effectiveness.

Table 2. Test matrix of FMVSS208 and NCAP tests

\begin{tabular}{|c|c|c|c|c|c|c|c|c|}
\hline Driver & \multicolumn{8}{|c|}{ FMVSS208 $40 \mathrm{~km} / \mathrm{h}$ Unbelted Tests } \\
\hline$\#$ & 1 & 2 & 3 & 4 & 5 & 6 & 7 & \\
\hline Test & 779 & 780 & 781 & 782 & 783 & 862 & 863 & \\
\hline $\begin{array}{l}\text { Driver } A B \\
\text { (Inflator) }\end{array}$ & $\begin{array}{l}\text { Baseline } \\
\text { (229kPa) }\end{array}$ & $\begin{array}{l}\text { Baseline } \\
\text { (229kPa) }\end{array}$ & $\begin{array}{c}\text { Depowered } \\
(174 \mathrm{kPa})\end{array}$ & $\begin{array}{c}\text { Depowered } \\
(174 \mathrm{kPa})\end{array}$ & $\begin{array}{l}\text { Baseline } \\
(229 \mathrm{kPa})\end{array}$ & $\begin{array}{c}\text { Depowered } \\
(125 \mathrm{kPa})\end{array}$ & $\begin{array}{c}\text { Depowered } \\
(125 \mathrm{kPa})\end{array}$ & \\
\hline IKB & N/A & $\mathrm{N} / \mathrm{A}$ & $\mathrm{N} / \mathrm{A}$ & N/A & In use & In use & In use & \\
\hline Driver & \multicolumn{8}{|c|}{ NCAP 56 km/h Belted Tests } \\
\hline$\#$ & 1 & 2 & 3 & 4 & 5 & 6 & 7 & 8 \\
\hline Test & 788 & 789 & 794 & 795 & 796 & 797 & 801 & 802 \\
\hline $\begin{array}{l}\text { Driver AB } \\
\text { (Inflator) }\end{array}$ & $\begin{array}{l}\text { Baseline } \\
(229 \mathrm{kPa})\end{array}$ & $\begin{array}{l}\text { Baseline } \\
(229 \mathrm{kPa})\end{array}$ & $\begin{array}{c}\text { Depowered } \\
(174 \mathrm{kPa})\end{array}$ & $\begin{array}{c}\text { Depowered } \\
(174 \mathrm{kPa})\end{array}$ & $\begin{array}{l}\text { Baseline } \\
(229 \mathrm{kPa})\end{array}$ & $\begin{array}{l}\text { Baseline } \\
(229 \mathrm{kPa})\end{array}$ & $\begin{array}{c}\text { Depowered } \\
(174 \mathrm{kPa})\end{array}$ & $\begin{array}{c}\text { Depowered } \\
(174 \mathrm{kPa})\end{array}$ \\
\hline IKB & $\mathrm{N} / \mathrm{A}$ & $\mathrm{N} / \mathrm{A}$ & $\mathrm{N} / \mathrm{A}$ & $\mathrm{N} / \mathrm{A}$ & In use & In use & In use & In use \\
\hline
\end{tabular}

Data was collected from the data acquisition system and recorded in IMPAX format, then converted to DIADEM (National Instruments, Austin, TX) format and filtered at SAE Channel Frequency Class $600 \mathrm{~Hz}$, following the SAE J-211 standard. Recorded time duration of the data started from $10 \mathrm{~ms}$ prior to time zero (i.e., onset time of sled pulse) and ended after $300 \mathrm{~ms}$. Three high-speed cameras documented the tests with maximum film coverage set at a frame rate of 1000 frames per second. For the NCAP tests, time shifting was performed to realign the signals of sled acceleration, as the input should be identical given the same test conditions. Correspondingly, all the tests data channels were shifted with a specified time frame.

Tibia index (TI) was applied as a criterion to quantify the lower limb injury severity. The tibia index addressed the axial compression and bending moment experienced at mid-shaft of the tibia (Mertz et al., 1993). The injury boundary of 1 was an engineering failure criterion and a $\mathrm{TI}>1$ indicated the possible occurrence of tibia fracture. A geometric adjustment of the tibia sagittal moment was performed for the 
upper and lower tibia moment measured from the Hybrid-III Denton leg. The adjustment compensated for the non-anatomical lower extremity geometry of the Hybrid-III dummy, which could result in an over-estimation of proximal tibia fracture (Zuby et al., 2001). The adjustment function was applied with the associated geometrical coefficients (Kuppa et al., 2001).

$$
\begin{aligned}
& M y_{\text {upper.adj }}=M y_{\text {upper.meas }}-\left(F z_{\text {upper }}\right)(0.02832) \\
& M y_{\text {lower.adj }}=M y_{\text {lower.meas }}+\left(F z_{\text {lower }}\right)(0.006398)
\end{aligned}
$$

Resultant tibia bending moment was then calculated after the adjustment of moment for the Hybrid-III leg. The revised TI was calculated for the Hybrid-III dummy leg, where $M_{r}$ is the resultant tibia moment, $F_{z}$ is the tibia axial compression force, $F_{c}$ and $M_{c}$ are the critical threshold values for force and moment in tibia index (Wellbourne et al., 1998; Zuby et al., 2001). A tibia index exceeding the critical value of 1 indicated the likelihood of fracture occurrence. A Wilcoxon rank-sum test was performed to compare the tibia indices difference between tests with bolster and tests with IKB, which was a nonparametric alternative to the twosample student t-test. A p value less than 0.05 indicated that the difference of tibia index was statistically significant from the group with IKB to the group with standard knee bolster.

$$
\begin{aligned}
& M_{r}=\sqrt{M_{x}^{2}+M_{y}^{2}} \\
& T I_{\text {HybridIII-50th }}=\frac{F_{z}}{F_{c}}+\frac{M_{r}}{M_{c}}=\frac{\left|F_{z}\right|}{35900}+\frac{M_{r}}{225}
\end{aligned}
$$

The injury risk function of tibia shaft fracture was applied to quantify the injury risk of AIS2+ lower limb injury for the $50^{\text {th }}$ percentile Hybrid-III dummy using the revised tibia index (Kuppa et al., 2001). A free body diagram was also applied to facilitate the analysis of kinetics in cases with and without inflatable knee bolster.

\subsection{Results}

\section{FMVSS208 Tests}

Deployment of the inflatable knee bolster was documented by the time-lapse photos from the highspeed cameras, with photo captures at an increment of $10 \mathrm{~ms}$ (Figure 18). The inflatable knee bolster fired at $18 \mathrm{~ms}$, and intruded out from the deployable instrument panel. At around $30 \mathrm{~ms}$, IKB contacted the anterior tibia shaft and knee surface, resulting in an ensuing extension of the lower limb, which altered the tibia into closer proximity with the floor pan during the initial deployment. The change of kinematics was demonstrated by the decrease in femur angle and tibia angle, compared to the tests with standard knee bolster in the photos. At around $70 \mathrm{~ms}$, forward excursion for the upper torso of the unbelted Hybrid III ATD was noticed, most likely attributable to the moment of inertia during the deceleration phase of the sled. This forward 
motion resulted in the accumulation of compression force at the femur and tibia, which also elevated the femur positioning relative to horizontal plane correspondingly. The inflatable knee bolster was deflated after full deployment and the dummy leg was wrapped over by the IKB at final stage.

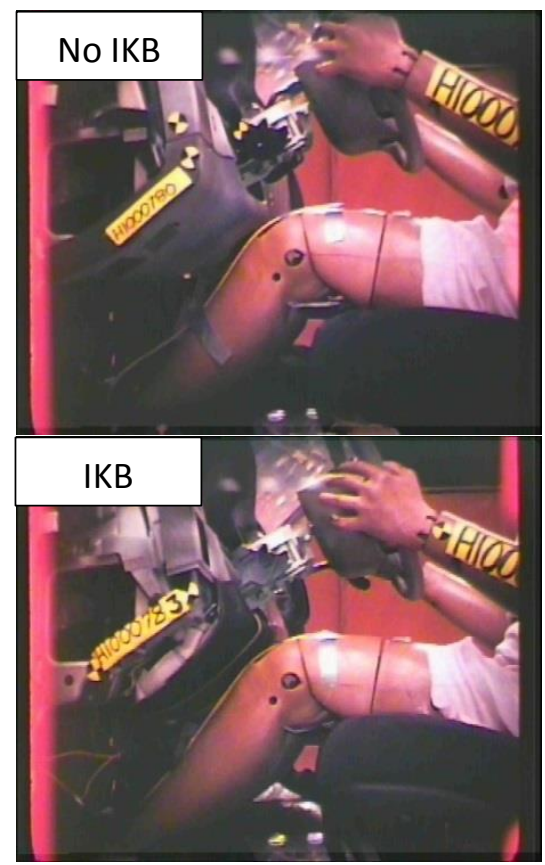

Time $=20 \mathrm{~ms}$

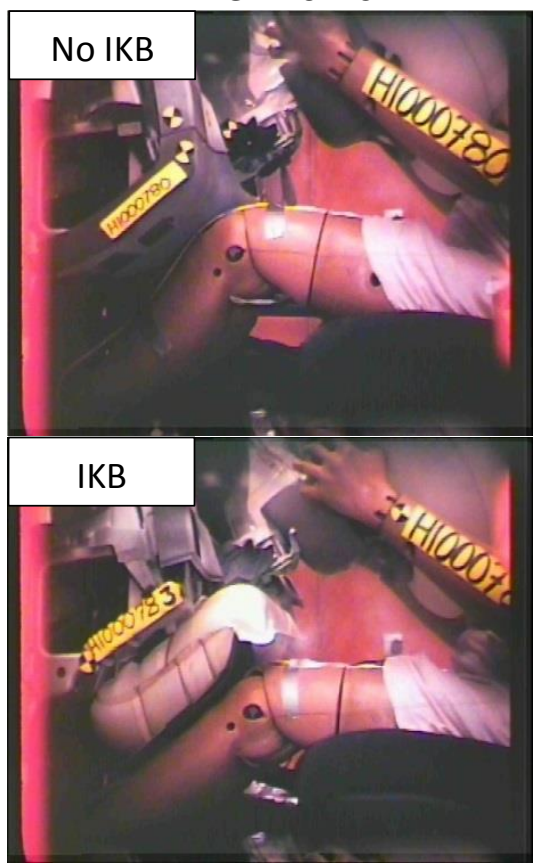

Time $=\mathbf{5 0} \mathrm{ms}$

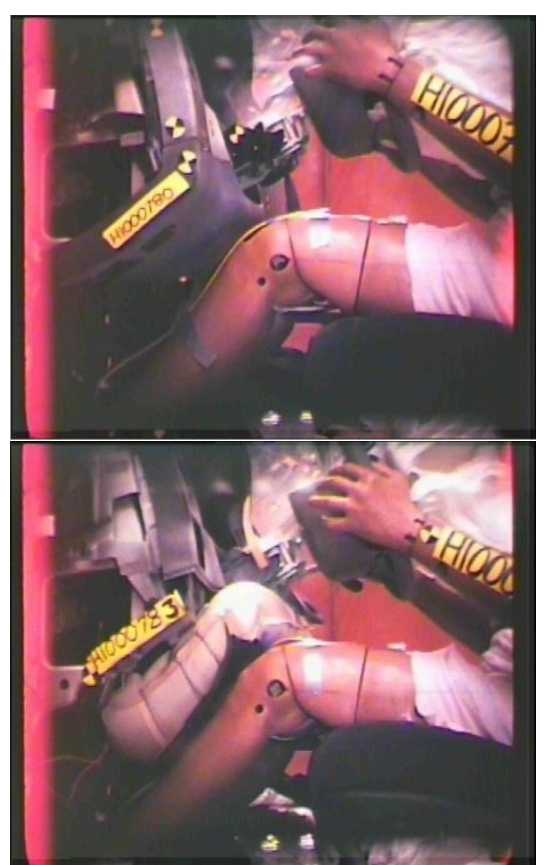

Time $=\mathbf{3 0} \mathrm{ms}$

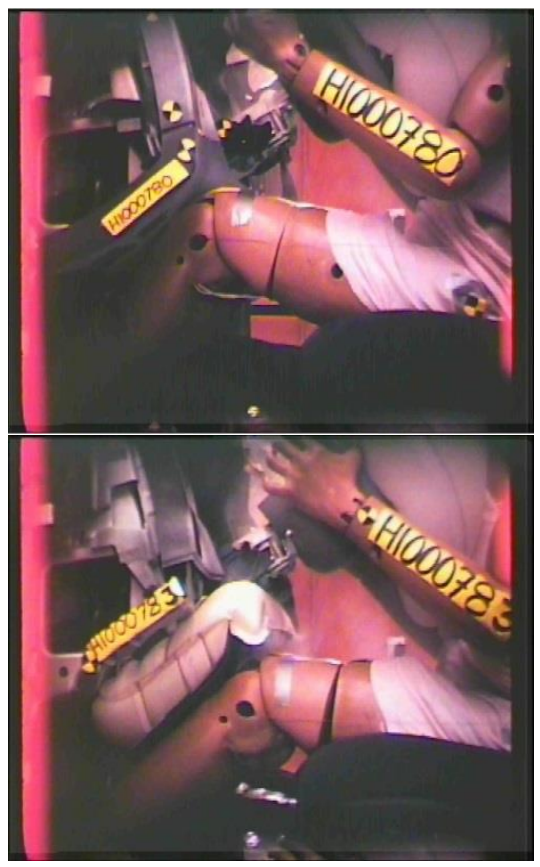

Time $=60 \mathrm{~ms}$

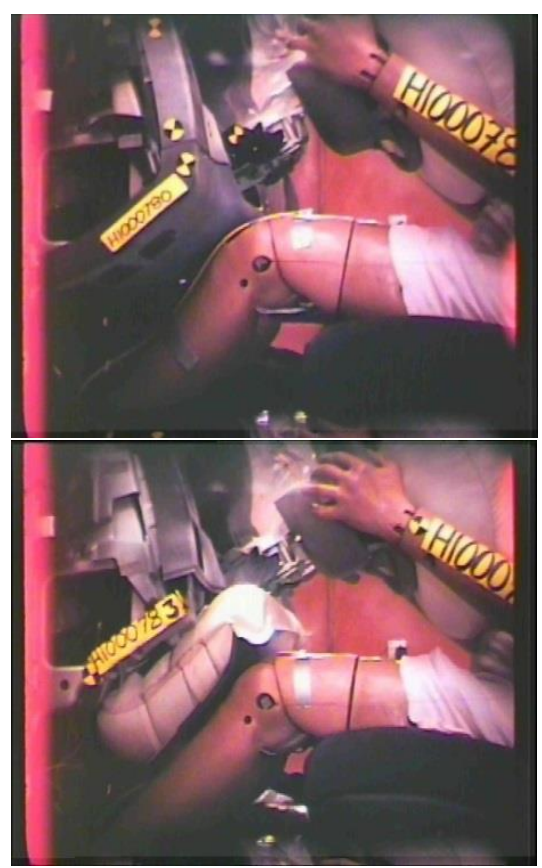

Time $=\mathbf{4 0} \mathrm{ms}$

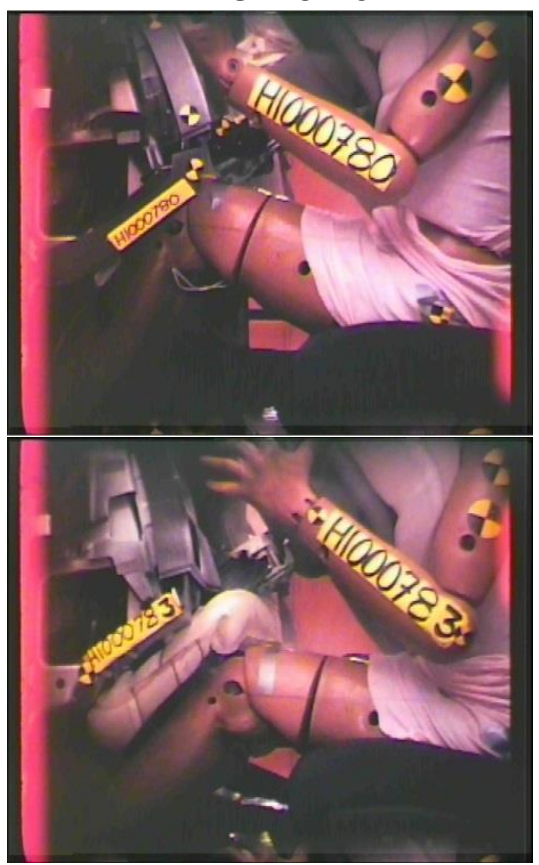

Time $=70 \mathrm{~ms}$ 


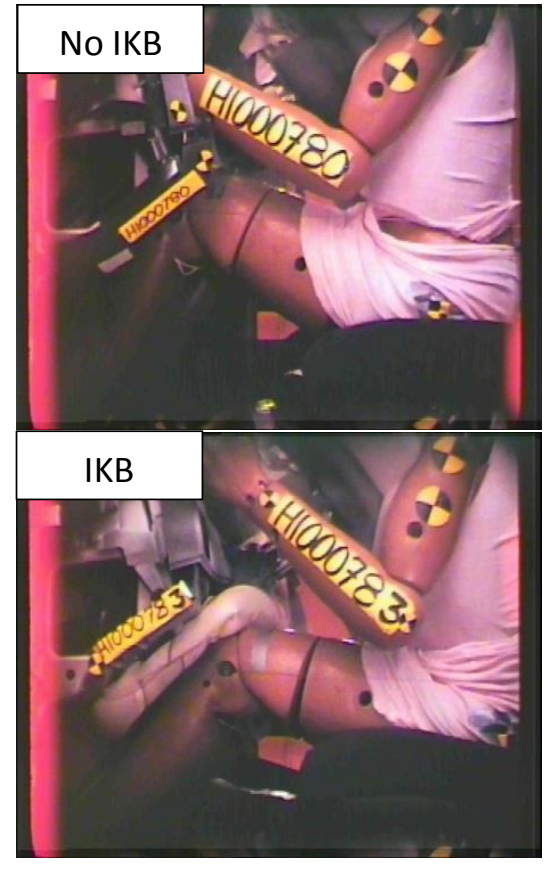

Time $=80 \mathrm{~ms}$

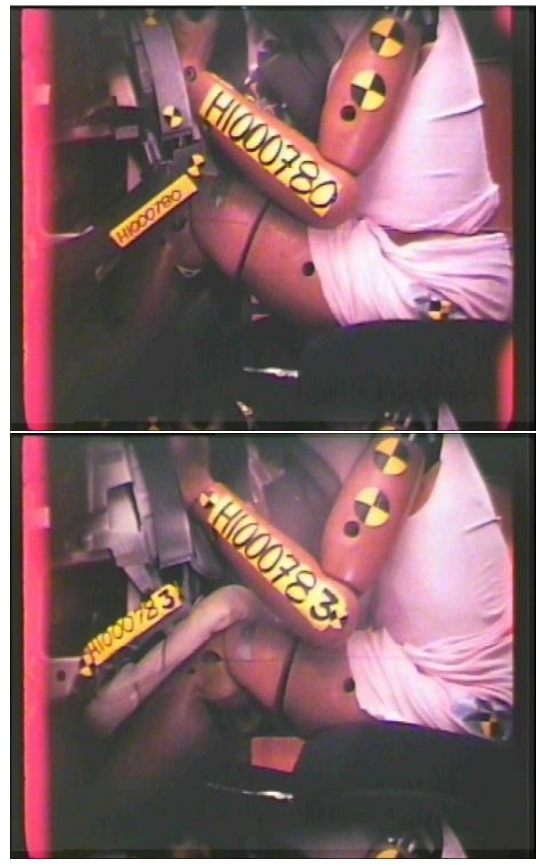

Time $=90 \mathrm{~ms}$

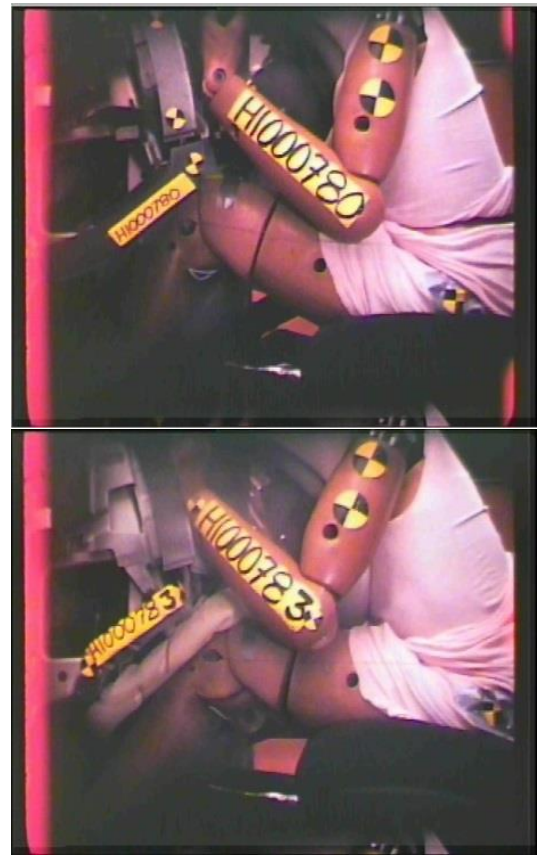

Time $=100 \mathrm{~ms}$

Figure 18. Time lapse photos of FMVSS208 unbelted tests with and without IKB

Figure 19 showed the input signal of sled pulse acceleration for the FMVSS 208 tests. The sled pulse indicated a bifurcated shape, with the first peak of $-15.3 \mathrm{G}$ occurring at $19.4 \mathrm{~ms}$, and the second peak of -29.4 $\mathrm{G}$ at $50.3 \mathrm{~ms}$.

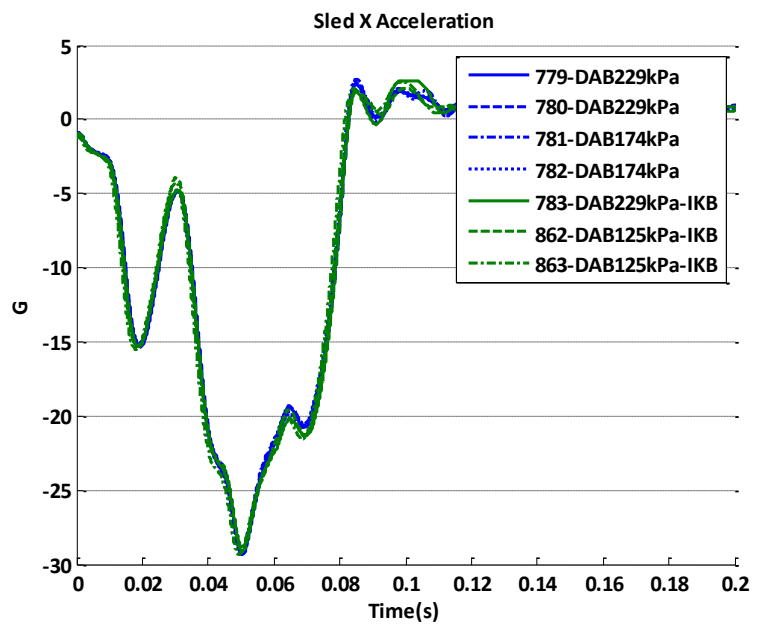

Figure 19. Sled acceleration pulse for FMVSS 208 tests

The phase difference in peak femur forces (i.e., $30 \mathrm{~ms}$ to $60 \mathrm{~ms}$ for IKB, $60 \mathrm{~ms}$ to $70 \mathrm{~ms}$ for standard bolster, respectively) was due to the femur early engagement with the IKB. Additionally, more oscillation occurred in tests with IKB, resulting from the reduction of stiffness due to IKB, as the airbag cushion deflated after deployment when the femur further engaged with the deployed bolster panel. Depowering of driver airbag inflator from $229 \mathrm{kPa}$ to $174 \mathrm{kPa}$ had little effect on the response of femur forces. As shown in Figure 20, femur force decreased significantly with IKB for both left and right extremities. 

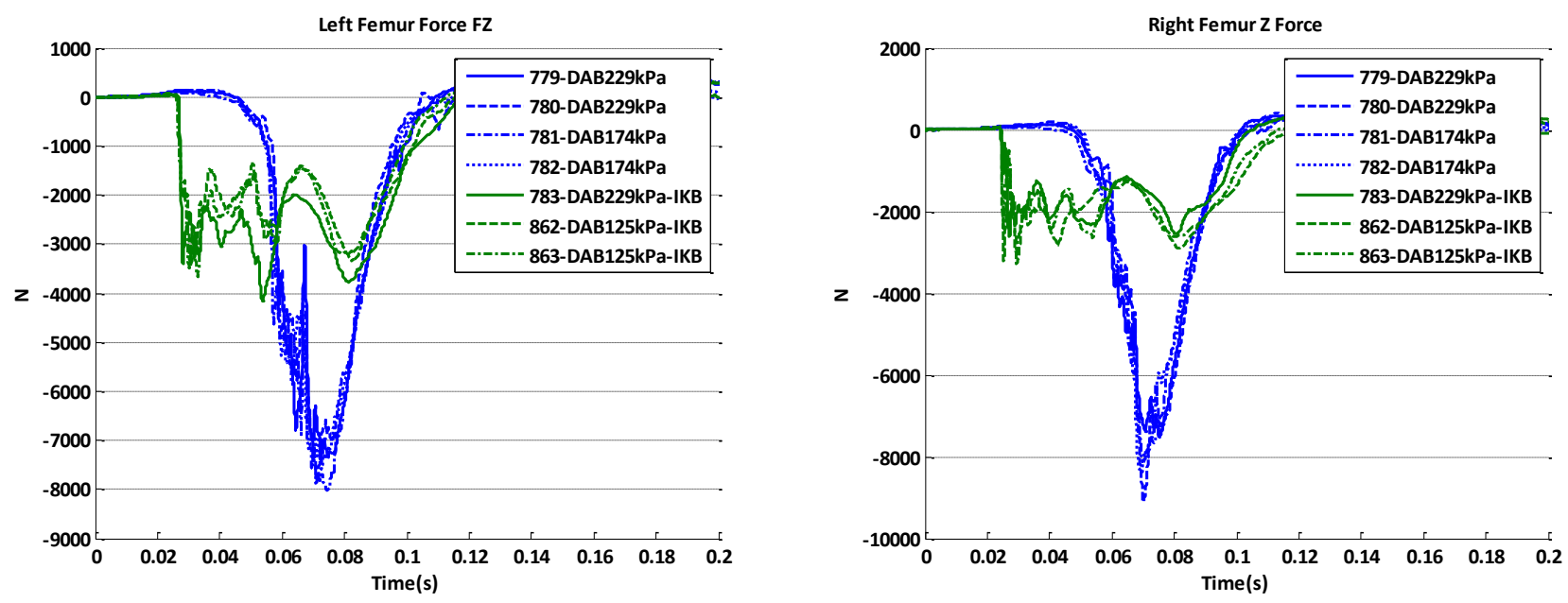

Figure 20. Femur response for FMVSS tests with and without IKB

IKB deployment resulted in a marginal decrease in left tibia compression force, with the average of peak compression force (FZ) decreased from $5816.2 \mathrm{~N}$ to $4373.3 \mathrm{~N}$ at left upper tibia, and from $6446.4 \mathrm{~N}$ to 4971.2 $\mathrm{N}$ at left lower tibia (Figure 21). Contact with the deploying IKB resulted in the first peak at tibia plateau region when upper tibia was pushed downward with the forward sliding of the foot. Rebounding interaction with the IKB corresponded to the decay phase of tibia force thereafter, with the second peak at tibia force denoted the compression originated from the forward excursion of the unbelted dummy. Maximum left upper tibia peak force (FZ) reached $5279.6 \mathrm{~N}$ in tests with IKB, which was close to the tibia plateau fracture threshold of $5.6 \mathrm{kN}$.

The lateral adduction of the left leg and slight rotation of the tibia region in IKB tests was captured by the oscillation in the tibia moment (MX). Moreover, IKB resulted in a positive tibia moment (MY) at early stage, which is the contributor for the change of lower extremity posture and reduction of tibia angles (Figure 22). The IKB deployed at tibia mid-shaft region, and resulted in both positive bending moment MY at proximal and distal tibia load cell. The change of bending moment direction at later stage resulted from the change in bimodal sled impulse shape, and the leg further engagement with the deflated IKB at a higher force acting point (i.e., from tibia mid-shaft to tibia plateau region). For tests with standard knee bolster, both proximal and distal tibia sustained a negative tibia bending moment MY. 

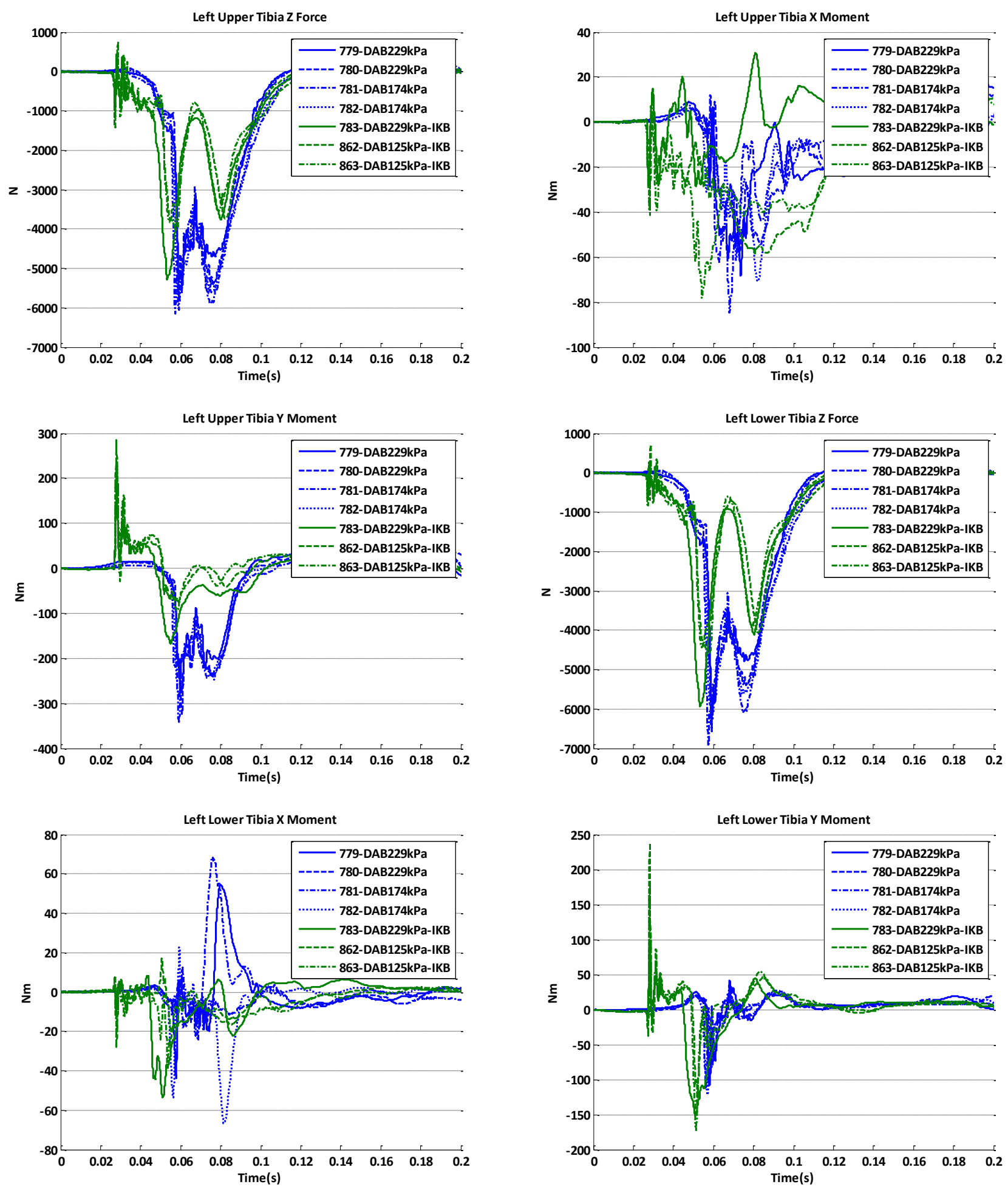

Figure 21: Left tibia response of FMVSS208 unbelted tests 


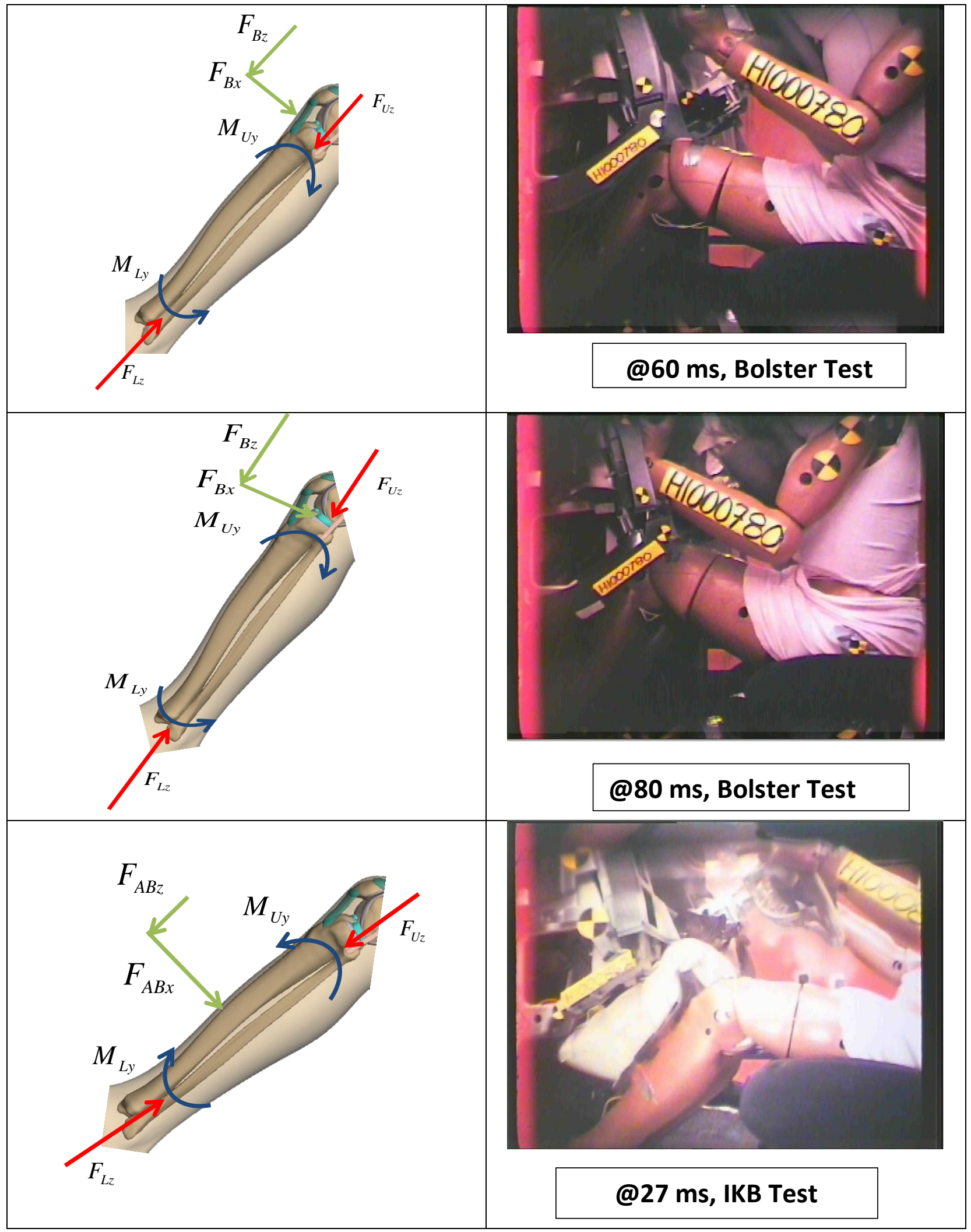




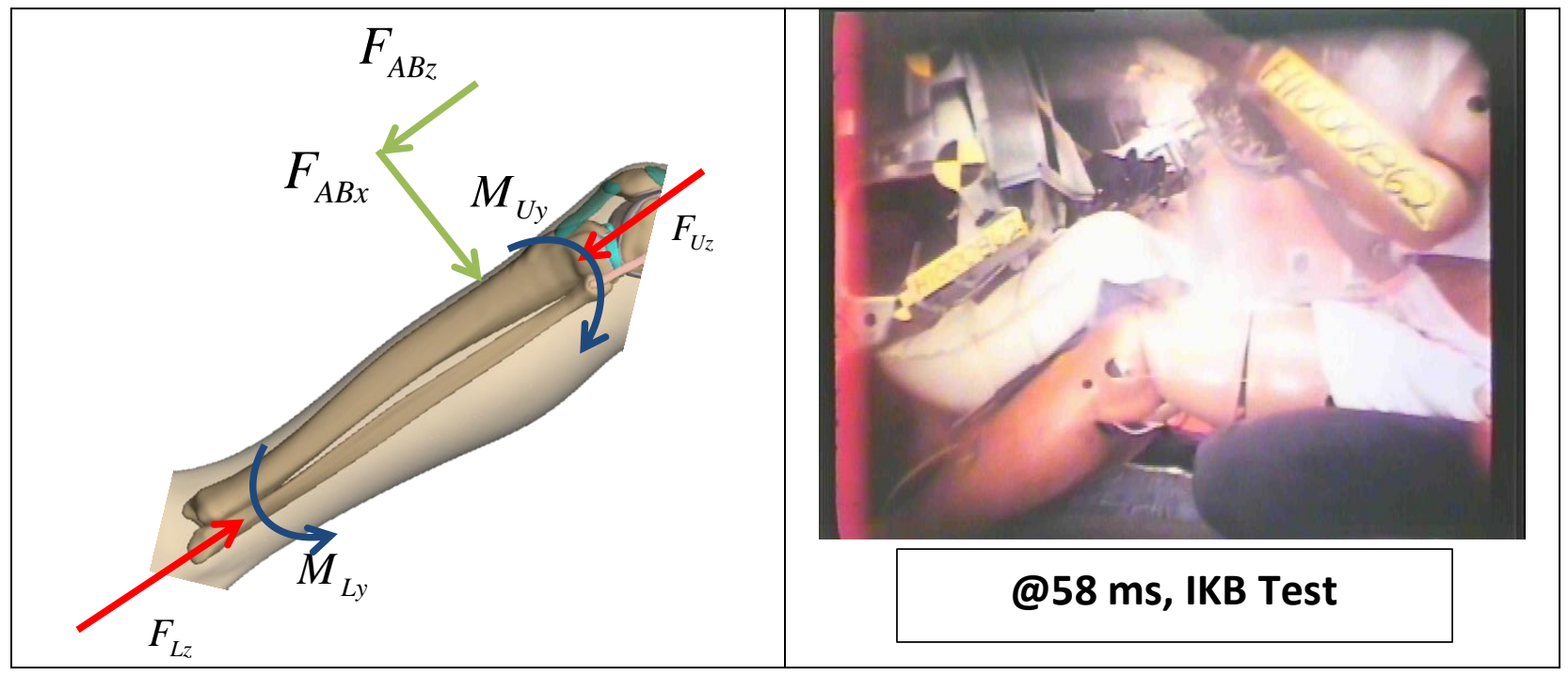

Figure 22. Comparison of kinetics for FMVSS tests with IKB and standard knee bolster

This finding indicated that knee airbag could possibly alter the direction of tibia moment MY, and expose the lower limb in an out-of-position posture.

The right upper tibia engaged with the deploying knee bolster at around $20 \mathrm{~ms}$, and compression force accumulated following the deployment of the IKB (Figure 23). The right upper tibia force with IKB slightly decreased from $4301.3 \mathrm{~N}$ to $3655.3 \mathrm{~N}$ on average, and the average of peak right lower tibia force decreased from $4813.5 \mathrm{~N}$ to $4089.1 \mathrm{~N}$, respectively. Both the direction of right tibia moment ( $\mathrm{MX}$ and $\mathrm{MY}$ ) was flipped due to deployment of IKB, corresponding to the abduction and flexion of the right lower limb. Additionally, the peak right upper tibia moment $\mathrm{MX}$ increased from $53.9 \mathrm{Nm}$ to $83.3 \mathrm{Nm}$ on average with the IKB presence.
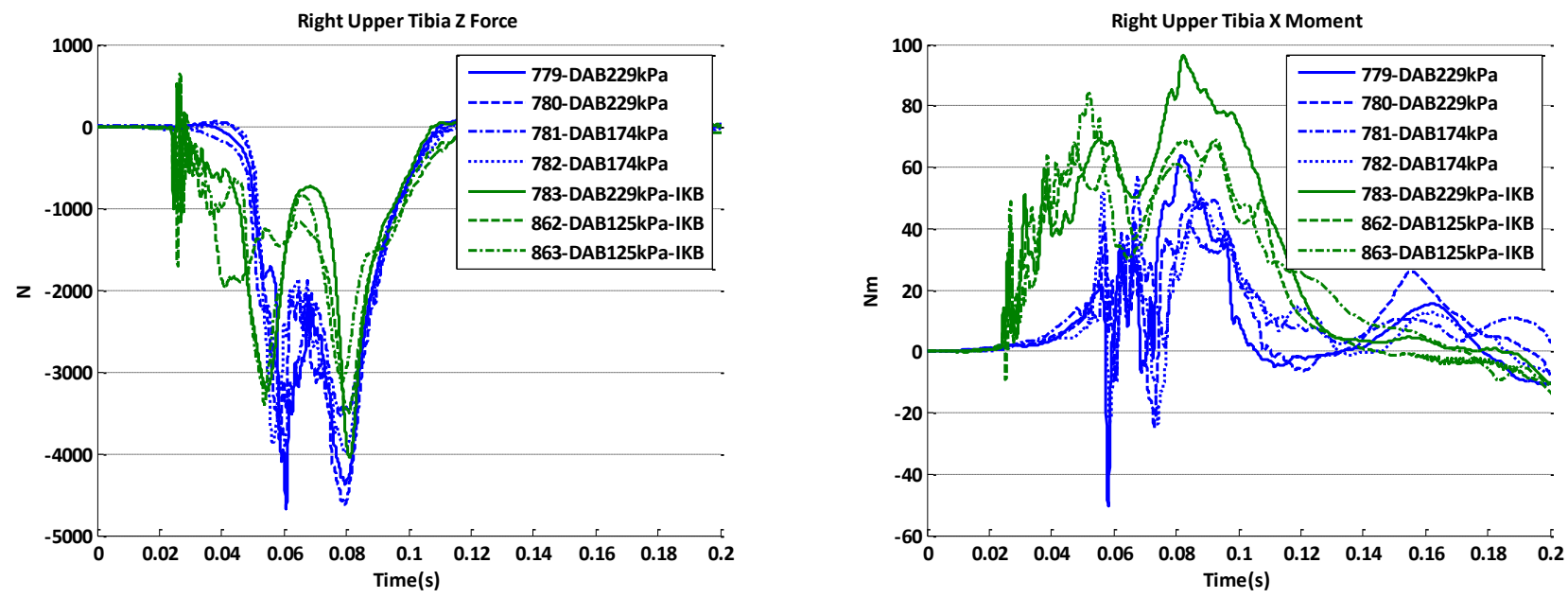

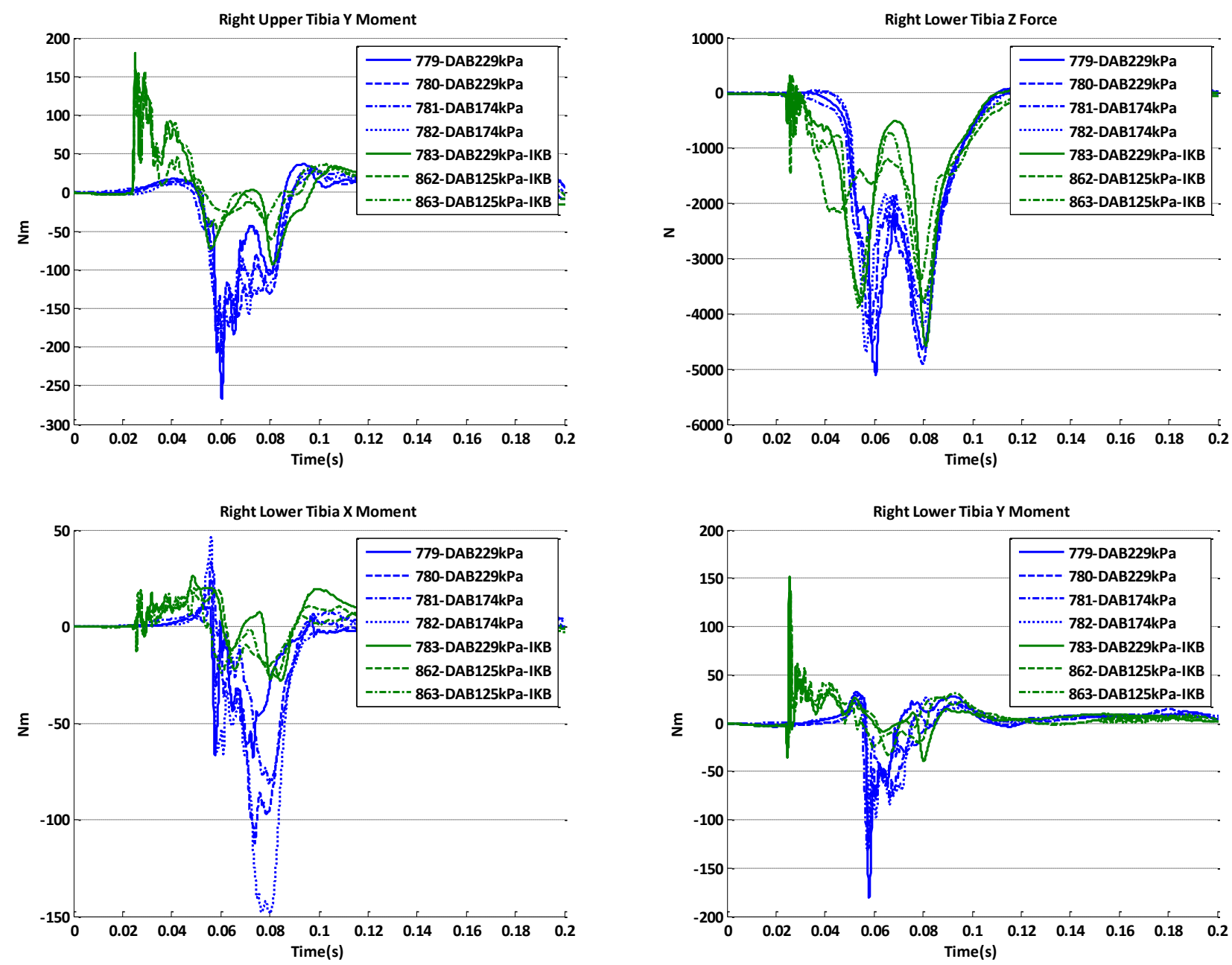

Figure 23: Right tibia response of FMVSS208 unbelted tests

The revised tibia index was calculated for left and right, lower and upper tibia for all the FMVSS208 tests (Table 3). On average, tibia index increased by $34.56 \%$ and $33.33 \%$ for left upper and left lower tibia index with IKB. For tests with IKB, right upper tibia index increased by $20.26 \%$ from 0.64 to 0.77 by average with the IKB. The only exception was the right lower tibia index, which decreased by $26.53 \%$ from 0.86 to 0.63 with IKB, compared to tests with standard bolster. The Wilcoxon Rank-sum Test indicated statistical significance in the tibia index difference in left upper tibia, left lower tibia and right lower tibia between the test group with IKB and the group with standard bolster. 
Table 3. Revised Tibia Index of FMVSS208 Unbelted Tests

\begin{tabular}{|c|c|c|c|c|c|}
\hline \multicolumn{6}{|c|}{ Revised Tibia Index (RTI) of FMVSS 208 Unbelted Tests } \\
\hline Test \# & Description & Left upper tibia & Left lower tibia & Right upper tibia & Right lower tibia \\
\hline 779 & Standard bolster, DAB 229kPa & 0.71 & 0.72 & 0.75 & 1.04 \\
\hline 780 & Standard bolster, DAB 229kPa & 0.89 & 0.68 & 0.64 & 0.83 \\
\hline 781 & Standard bolster, DAB 174kPa & 0.94 & 0.73 & 0.50 & 0.78 \\
\hline 782 & Standard bolster, DAB 174kPa & 0.73 & 0.79 & 0.66 & 0.78 \\
\hline 783 & IKB, DAB 229kPa & 1.01 & 0.98 & 0.81 & 0.68 \\
\hline 862 & IKB, DAB 125kPa & 1.21 & 1.06 & 0.75 & 0.60 \\
\hline 863 & IKB, DAB 125kPa & 1.08 & 0.88 & 0.74 & 0.61 \\
\hline Ave. TI of standard bolster (mean $\pm S D$ ) & $0.82 \pm 0.11$ & $0.73 \pm 0.05$ & $0.64 \pm 0.10$ & $0.86 \pm 0.12$ \\
\hline \multicolumn{7}{|c|}{ Ave. TI of IKB (mean $\pm S D)$} & $1.10 \pm 0.10$ & $0.97 \pm 0.09$ & $0.77 \pm 0.04$ & $0.63 \pm 0.04$ \\
\hline \multicolumn{7}{|c|}{ Wilcoxon Rank-sum Test (p-value) } & $0.0339 *$ & $0.0339 *$ & 0.1084 & $0.0323^{*}$ \\
\hline
\end{tabular}

\section{NCAP tests}

The following time-lapsed photos documented the NCAP tests with and without IKB (Figure 24). IKB deployed against the anterior tibia shaft and plateau region after firing at $18 \mathrm{~ms}$, and engaged with the knee and femur region. Seatbelt for the NCAP tests constrained the pelvis forward sliding compared to FMVSS208 tests and consequently, the rebounding of the femur impact was reduced. IKB also provided early engagement with both aspects of the tibia, which resulted in a phase difference of approximately $20 \mathrm{~ms}$ in tibia compression force. As denoted in Figure 24, IKB significantly reduced the femur angle and tibia angle during the interaction, and altered the lower limb in a relatively more extended positioning compared to conventional knee bolster.

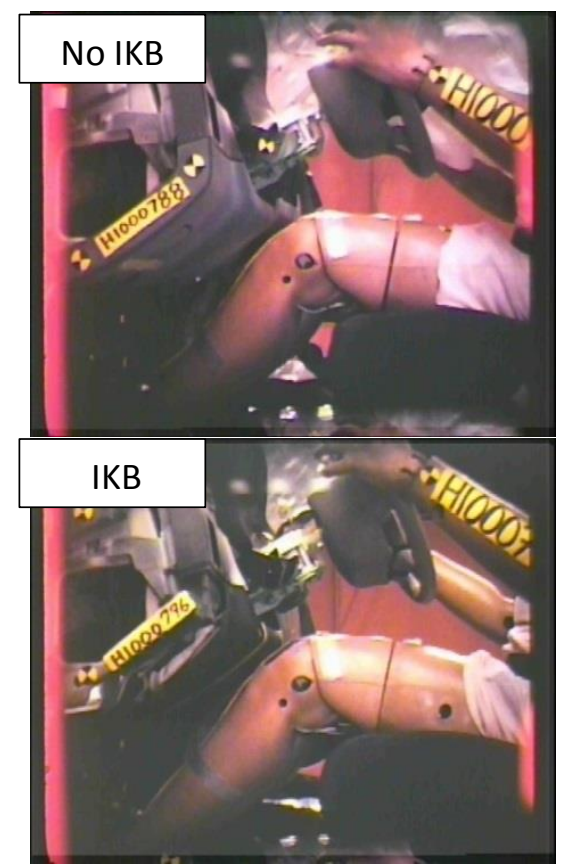

Time $=\mathbf{2 0} \mathrm{ms}$

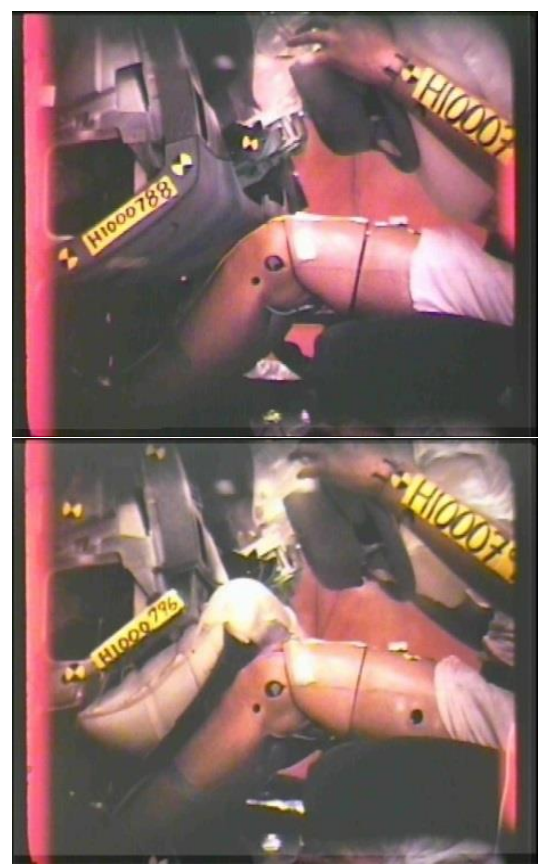

Time $=30 \mathrm{~ms}$

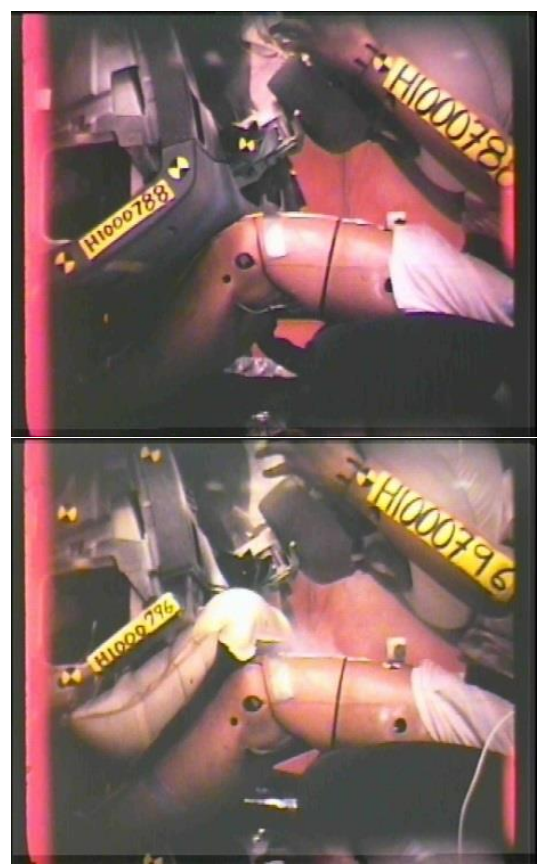

Time $=\mathbf{4 0} \mathrm{ms}$ 


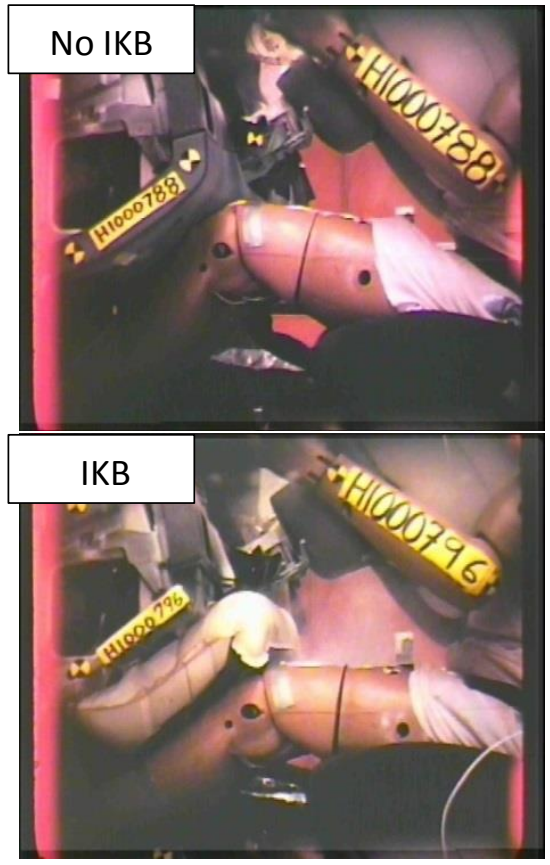

Time $=50 \mathrm{~ms}$

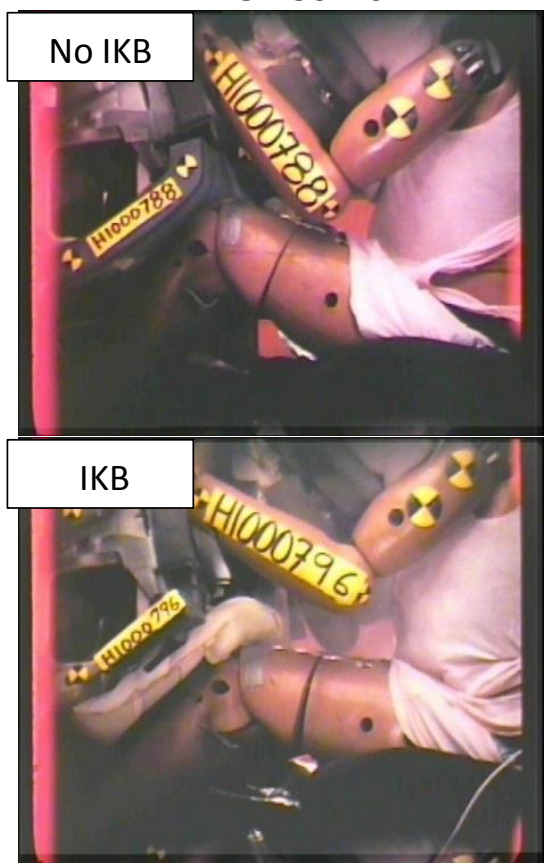

Time $=80 \mathrm{~ms}$

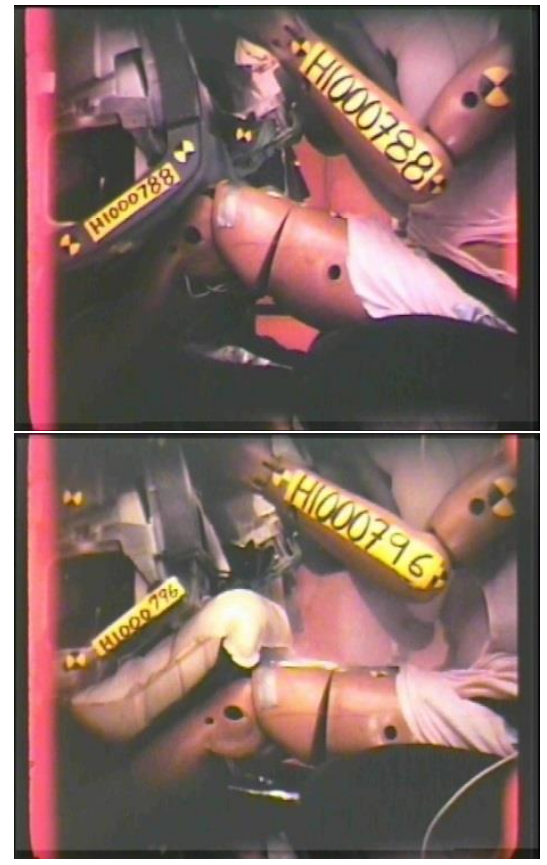

Time $=60 \mathrm{~ms}$

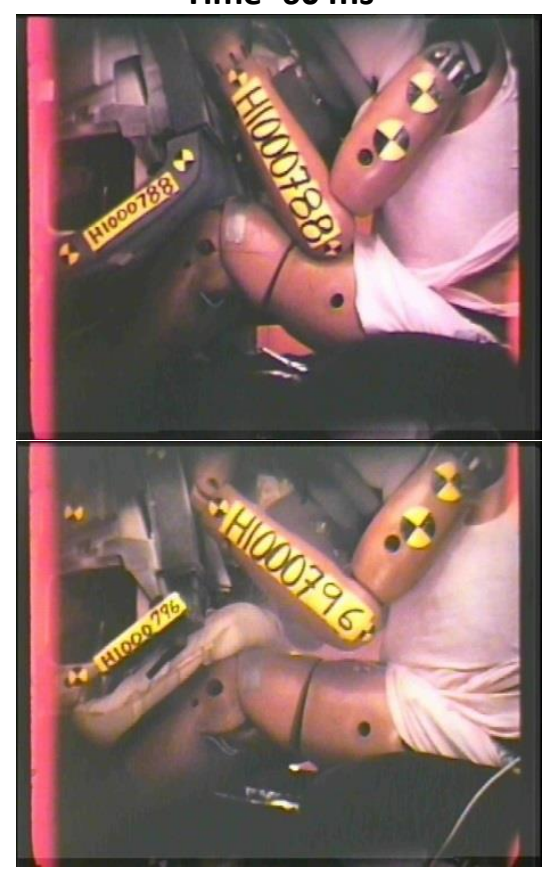

Time $=90 \mathrm{~ms}$

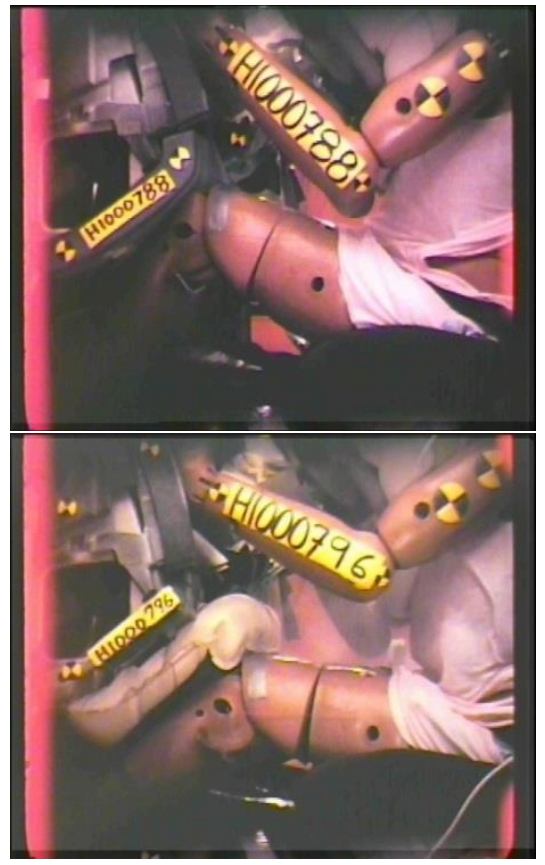

Time $=70 \mathrm{~ms}$

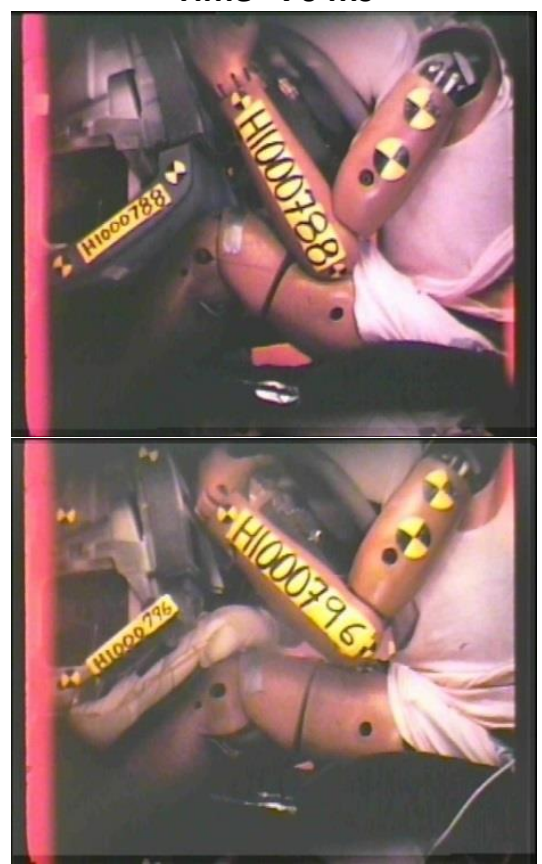

Time $=100 \mathrm{~ms}$

Figure 24. Time lapse photos of belted NCAP tests with and without IKB

The pulse signal for the sled acceleration of all NCAP tests was shown in Figure 25, with the peak sled acceleration occurred at 50.4 ms with a magnitude of $-31.9 \mathrm{G}$. 


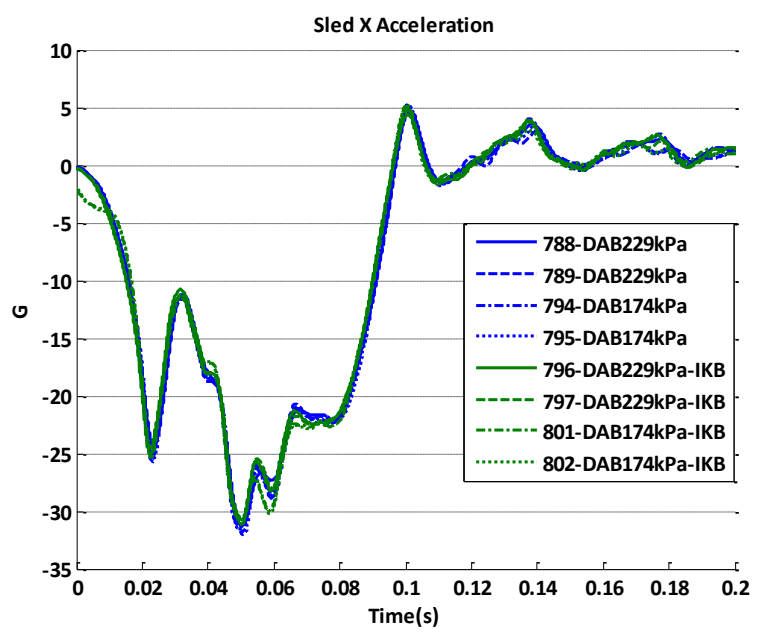

Figure 25. Sled acceleration pulse for NCAP tests

For belted $50^{\text {th }}$ Hybrid-III ATD, right femur force slightly decreased with IKB, from $2970.5 \mathrm{~N}$ to $2945.4 \mathrm{~N}$ on average (Figure 26). Left femur force, however, increased with the IKB by $35.5 \%$, mainly due to the late deployment of IKB in test 797 (IKB actually deployed at 29ms after set firing time at 18ms), which culminated to a peak force of $5737.3 \mathrm{~N}$. Overall, the femur forces were comparable in magnitude for tests with IKB to those with the standard bolster.
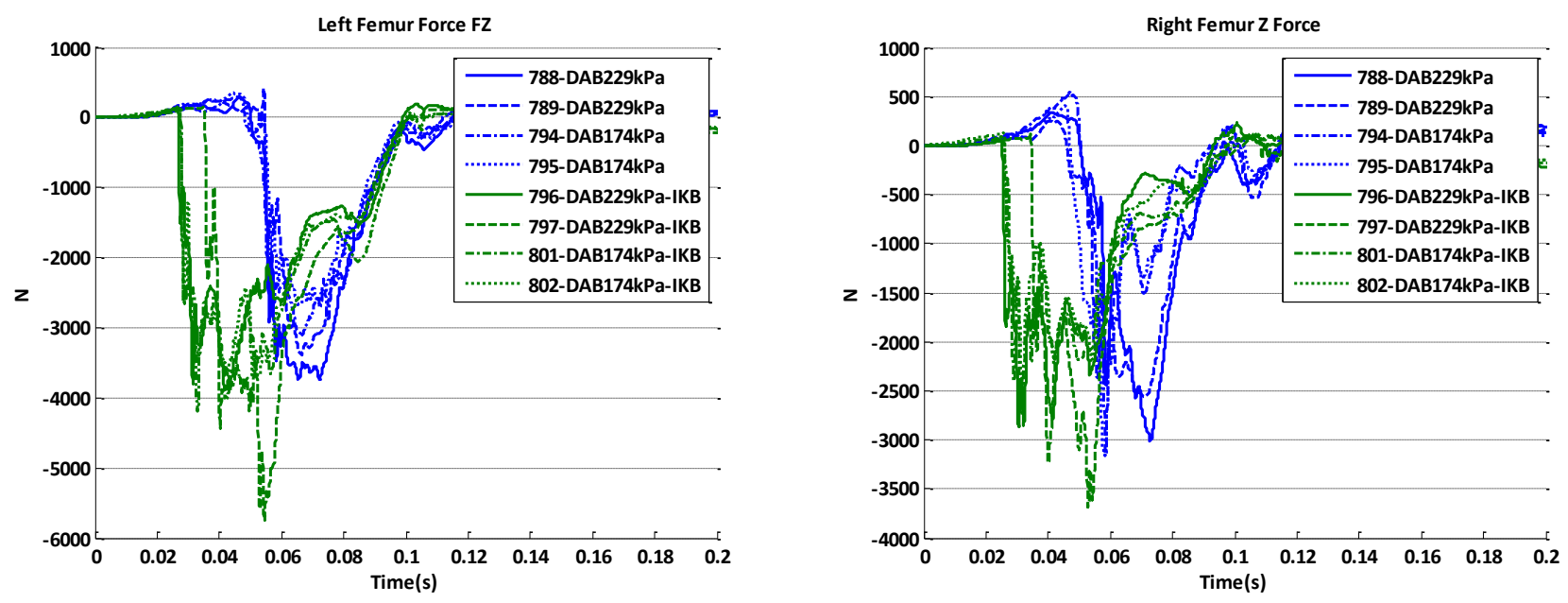

Figure 26. Femur response for NCAP tests with and without IKB

Two tests (test 796 and test 802) with IKB had a decrease in the left upper tibia compression force, although the left upper tibia force (FZ) slightly increased with the inflatable knee bolster on average (Figure 28). Furthermore, IKB also resulted in the change of direction in the tibia bending moment MY, which moved the ankle forward and knee rearward to a more extended posture, relative to the original lower limb alignment (Figure 27). As a result of this bending effect, the proximal tibia had a positive bending moment and rotated at around $28 \mathrm{~ms}$ following the deployment of the IKB. For test 797 with IKB, maximum left upper tibia 
axial compression force reached $6780 \mathrm{~N}$ in the upper tibia, and $7926 \mathrm{~N}$ in the lower tibia, which was close to the compressive force criterion of $8 \mathrm{kN}$ for tibia plateau fracture (Funk et al., 2004). This highest compression force could possibly be a combination of late IKB deployment in this case and initial left foot positon. The IKB deployed $10 \mathrm{~ms}$ later than the preset firing time, which applied a higher bending moment to the tibia that changed the tibia to a more extended driving posture. Additionally, the foot was not completely locked on the floor with full contact, and a fair amount of foot sliding was driven during the IKB deployment, as observed from the test videos. As a result, higher amount of deceleration force from the toe pan region transmitted to the tibia in this test.

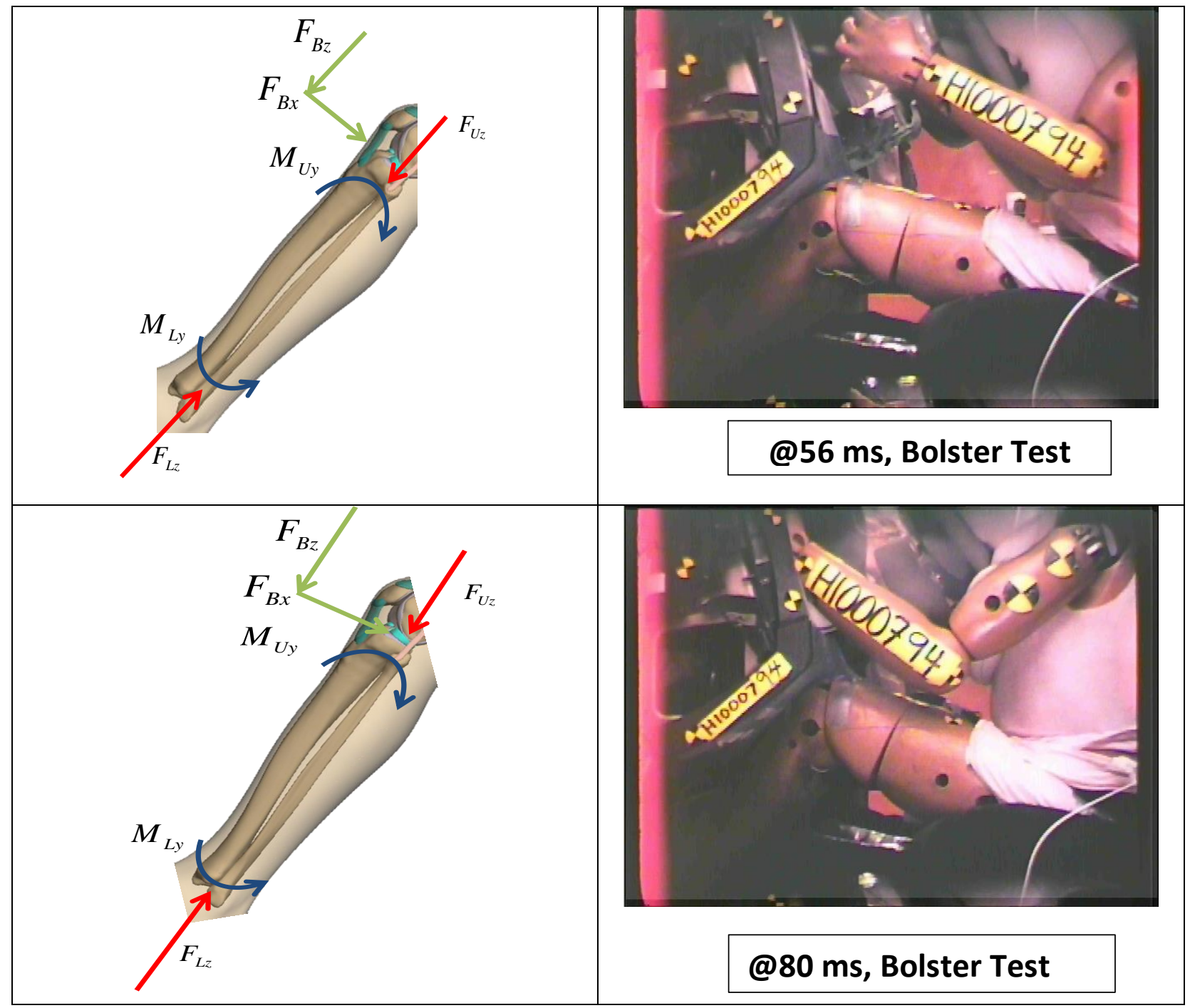




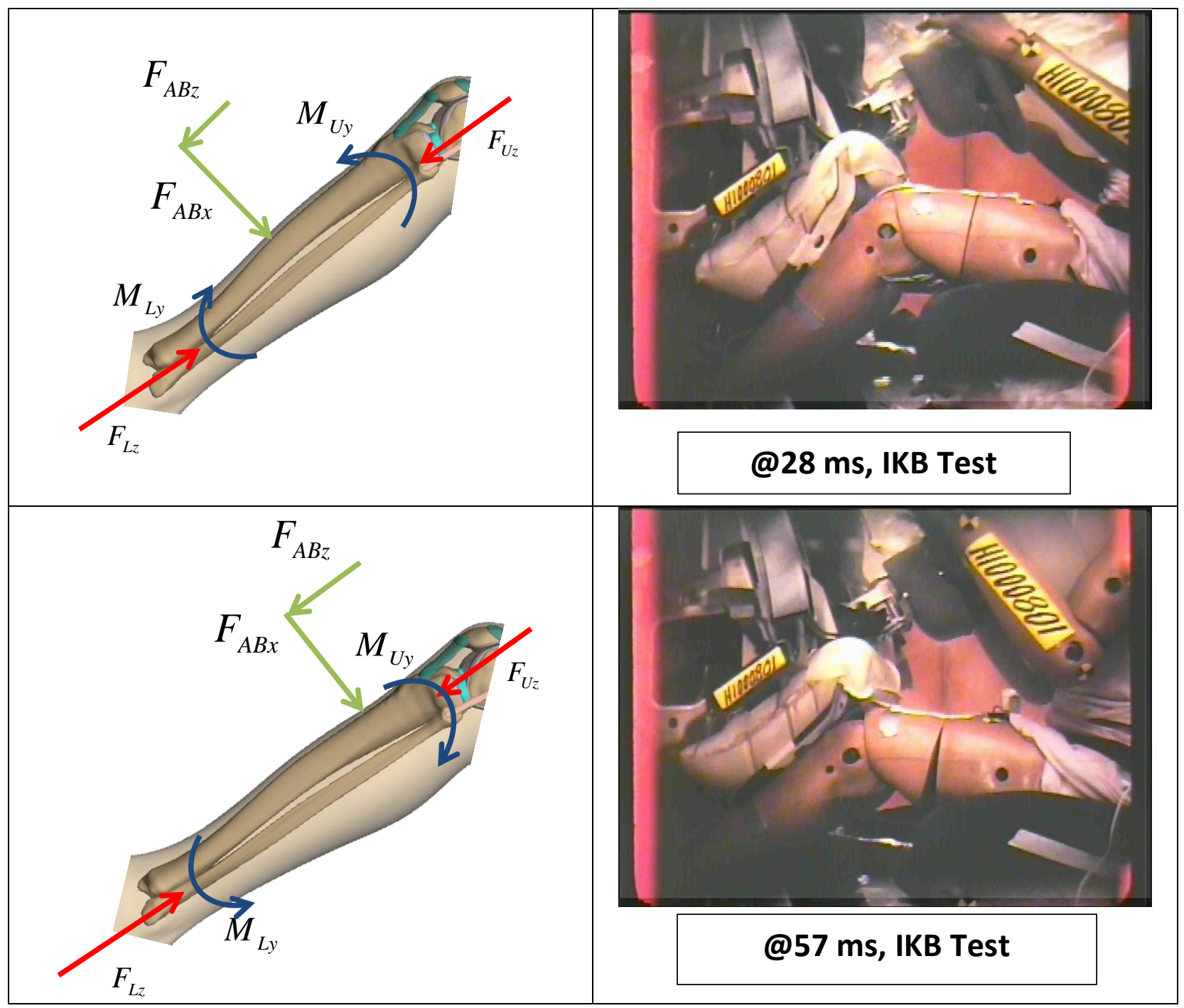

Figure 27. Comparison of kinetics for NCAP tests with IKB and standard knee bolster
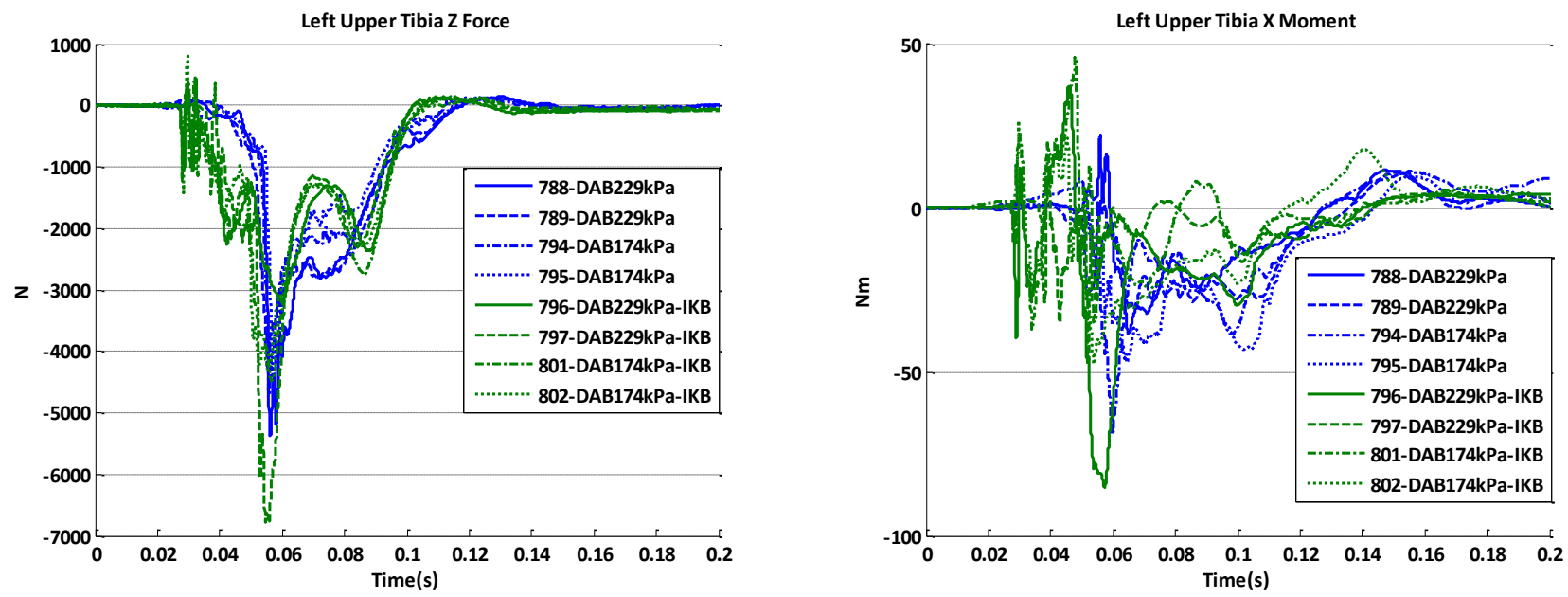

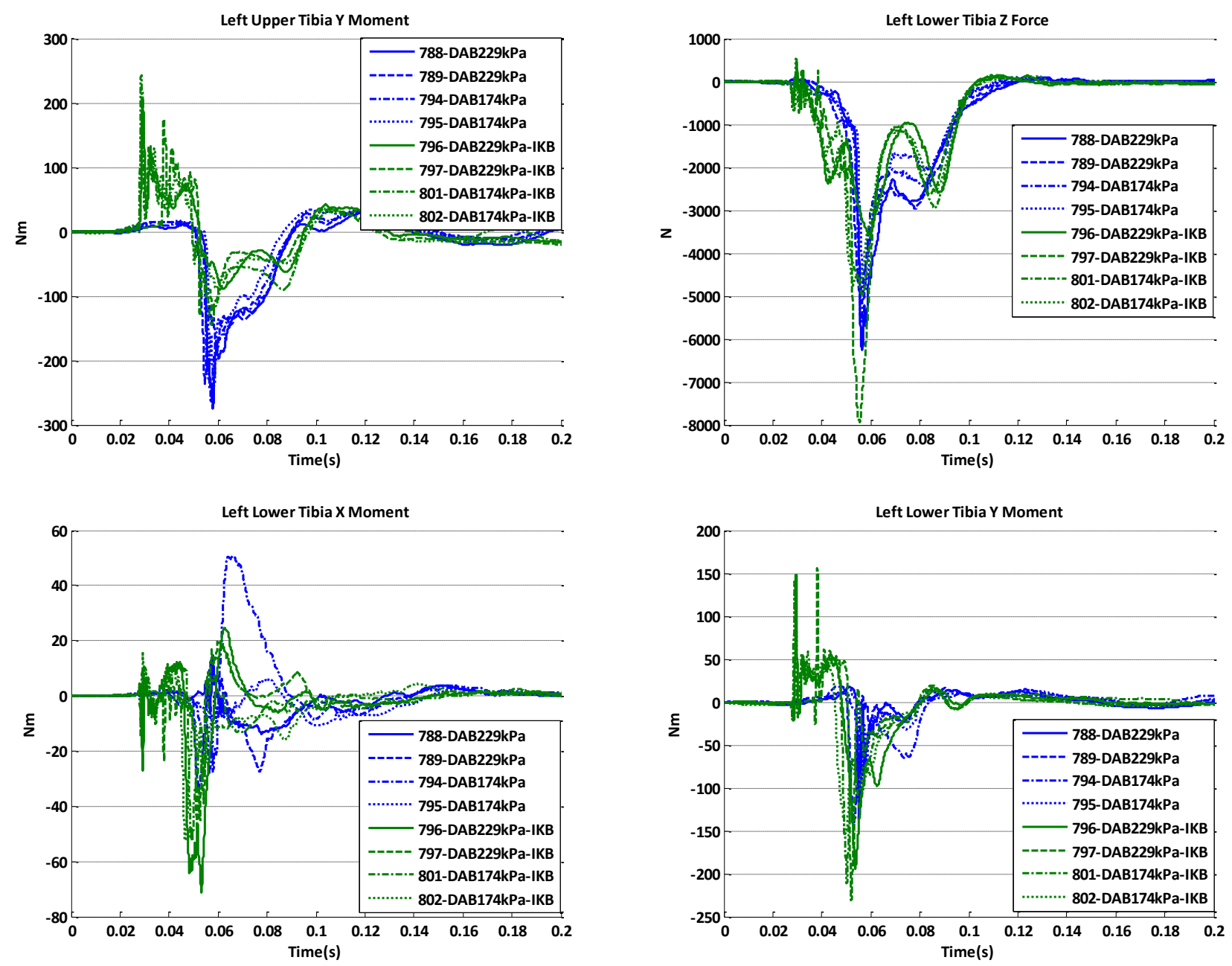

Figure 28. Left tibia response for NCAP tests with and without IKB

Marginal decrease of the right upper tibia compression force was observed with IKB, with the average decreased by around $200 \mathrm{~N}$ (Figure 29). The accompanied lateral sliding resulted in the abduction of the right upper tibia and a much higher tibia moment MX. The deployment of IKB was also responsible for the positive tibia MY moment at both right upper tibia and right lower tibia regions.
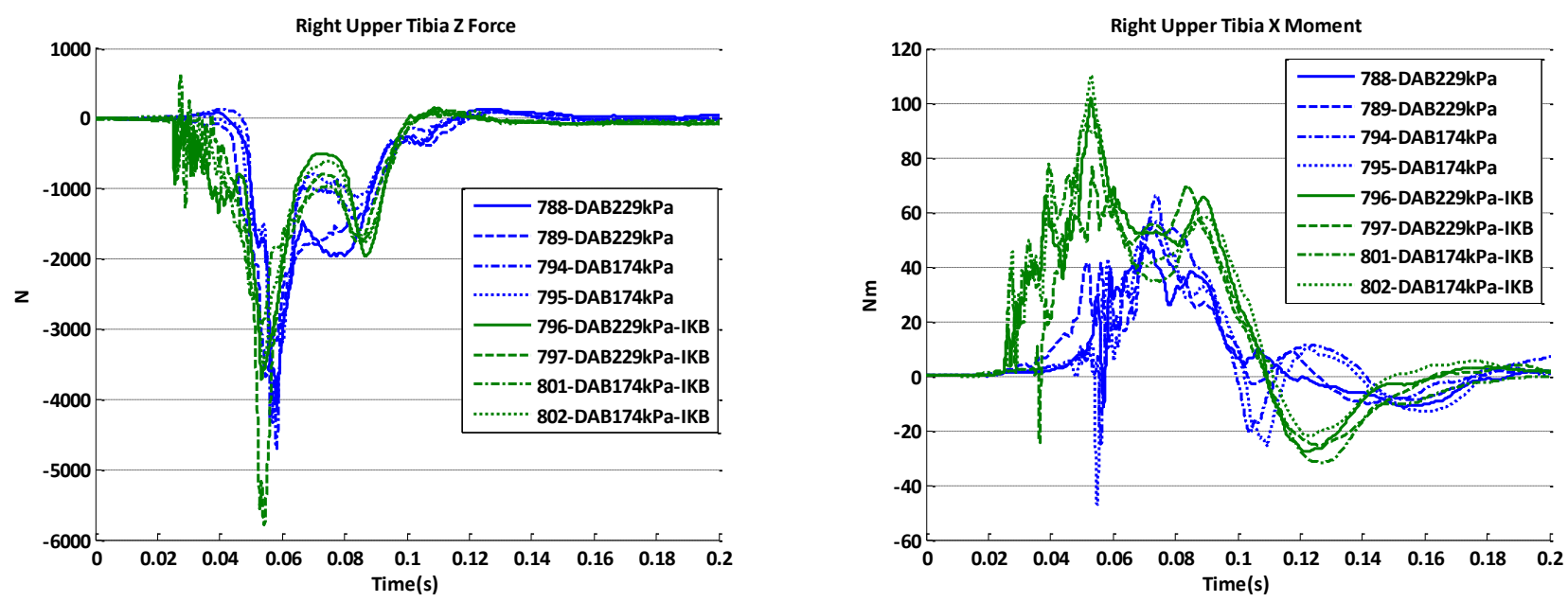

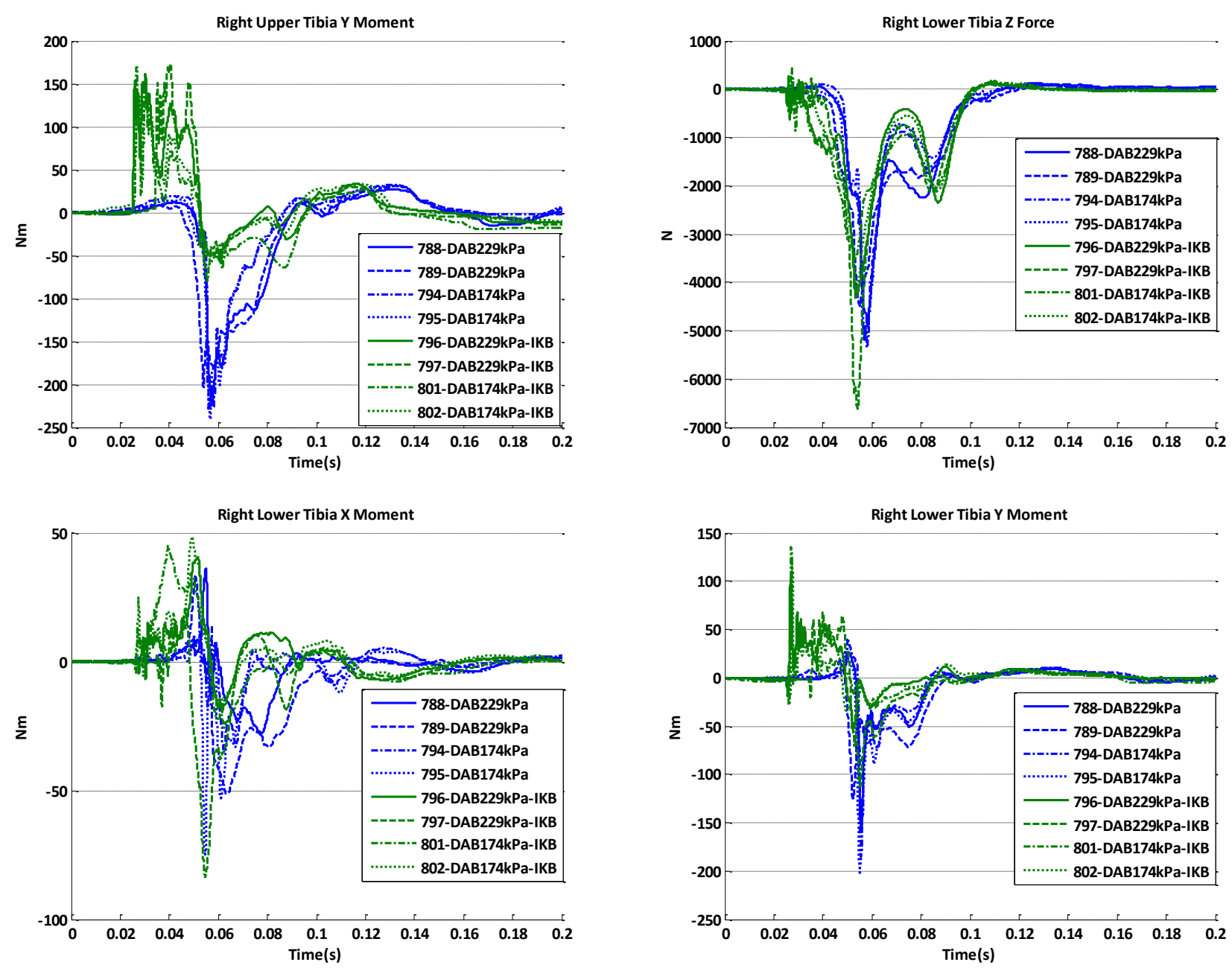

Figure 29. Right tibia response for NCAP tests with and without IKB

Revised tibia index indicated that with the presence of IKB, the average of left upper tibia index increased from 0.72 to 0.97 (Table 4). Left lower tibia and right upper tibia index also increased by $41.0 \%$ and 29.4\% correspondingly with IKB. Right lower tibia index, however, decreased from 0.93 to 0.64 by average. Additionally, results from the Wilcoxon Rank-sum test indicted the increase of tibia index from the test group with standard knee bolster to the group with IKB was statistically significant ( $p$-value $<0.05$ ). The elevated tibia index reflected the potential adverse effect of the IKB, and the increased injury risk of the lower extremity injuries. 
Table 4. Revised Tibia Index of NCAP Belted Tests

\begin{tabular}{|c|c|c|c|c|c|}
\hline \multicolumn{5}{|c|}{ Revised Tibia Index (RTI) of NCAP Belted Tests } \\
\hline Test \# & Description & Left upper tibia & Left lower tibia & Right upper tibia & Right lower tibia \\
\hline 788 & Standard bolster, DAB 229kPa & 0.73 & 0.83 & 0.61 & 0.89 \\
\hline 789 & Standard bolster, DAB 229kPa & 0.70 & 0.81 & 0.59 & 0.71 \\
\hline 794 & Standard bolster, DAB 174kPa & 0.76 & 0.68 & 0.63 & 1.03 \\
\hline 795 & Standard bolster, DAB 174kPa & 0.67 & 0.68 & 0.69 & 1.09 \\
\hline 796 & IKB, DAB 229kPa & 0.89 & 1.05 & 0.80 & 0.48 \\
\hline 797 & IKB, DAB 229kPa & 0.81 & 1.23 & 0.98 & 0.92 \\
\hline 801 & IKB, DAB 174kPa & 1.10 & 1.10 & 0.77 & 0.60 \\
\hline 802 & IKB, DAB 174kPa & 1.08 & 0.85 & 0.71 & 0.56 \\
\hline Ave. TI of standard bolster (mean $\pm S D)$ & $0.72 \pm 0.04$ & $0.75 \pm 0.08$ & $0.63 \pm 0.04$ & $0.93 \pm 0.17$ \\
\hline \multicolumn{2}{|c|}{ Ave. TI of IKB (mean \pm SD) } & $0.97 \pm 0.14$ & $1.06 \pm 0.16$ & $0.82 \pm 0.12$ & $0.64 \pm 0.19$ \\
\hline \multicolumn{2}{|c|}{ Wilcoxon Rank-sum Test (p-value) } & $0.0209^{*}$ & $0.0202^{*}$ & $0.0209 *$ & 0.0833 \\
\hline
\end{tabular}

The tibia index consists of an interaction criterion that combines the axial loading and bending, and was formulated at the mid-shaft tibia region (Funk et al., 2004). The human tibia diaphysis generally bows anteriorly and medially, and a substantial moment could also be induced by pure axial compressive loading, which creates tensile stress on the anterior side of the tibia, and compressive stress on the posterior side (Figure 30). The magnitude of this induced moment was equal to the axial load multiplied by the perpendicular distance between axial loading path and the centroid of the bone. This resulted in the difference in the combined stress of the tibia for tests with IKB and tests with standard bolster. For tests with IKB, deployment of the IKB resulted in a positive bending moment at proximal and distal tibia, which is subtractive with the negative bending moment induced from the axial compressive loading. However, the bending moment $M_{U y}$ and $M_{L y}$ later altered the direction and flipped to negative moment in IKB tests, which resulted in an additive effect with the axial loading induced bending moment $M_{\text {induced }}$. Correspondingly, for standard bolster test, the contact between knee bolster and the knee created a negative bending moment $M_{U y}$ and $M_{L y}$, which is consistent in the direction with the axial loading induced bending moment $M_{\text {induced }}$ throughout the whole crash event. 


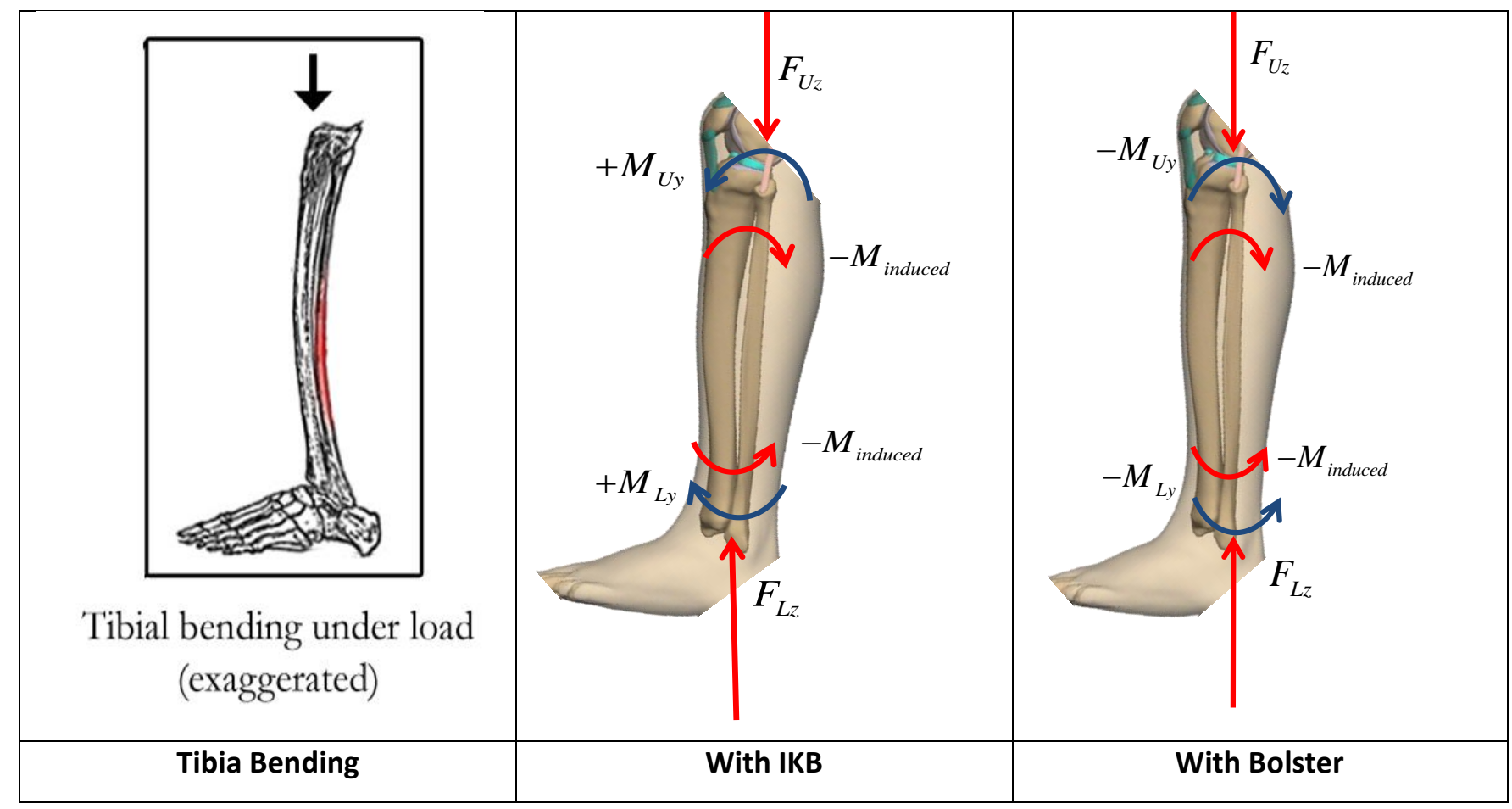

Figure 30. Combined stress analysis of the tibia for tests with IKB and bolster

\subsection{Free Body Diagram Analysis of Kinematics}

A key factor to understand the response of lower extremity under the knee airbag deployment was the kinetics. A simplified free body diagram was used to facilitate this illustration and cross compare the difference between inflatable knee bolster and standard bolster. The lower limb was simplified as two beam elements connected by pin joints for current analysis (Figure 31). The local coordinate system was applied in the free body analysis, with positive $X$ direction pointing leftward (perpendicular to the tibia), positive $Z$ direction pointing downward (parallel to the tibia) and positive $Y$ direction pointing inboard. The tibia was truncated from the lower tibia load cell to upper tibia load cell for analysis, with axial force $F_{U z}$ and $F_{L z}$, shearing force $F_{U x}$ and $F_{L x}$, as well as bending moment $M_{U y}$ and $M_{L y}$ denoted in the free body diagram. $F_{A B z}$ and $F_{A B x}$ represented the decomposition components of knee airbag deployment force at the tibia shaft region. The direction of all the force and moment were drawn following the polarity of SAE J-211 standard (SAE, 1995). $\alpha$ represented the tibia angle, which was defined as the angle between the tibia axial line and the horizontal plane; $\theta$ was the knee bolster angle (i.e., the deployment direction of the inflatable knee bolster surface); and $\beta$ denoted the femur angle (i.e., the angle between femur line and horizontal plane). The distance between upper tibia load cell and lower tibia load cell was marked as $L_{T i b}$, and $l$ referred to the distance between ankle joint to knee bolster force point of action. 


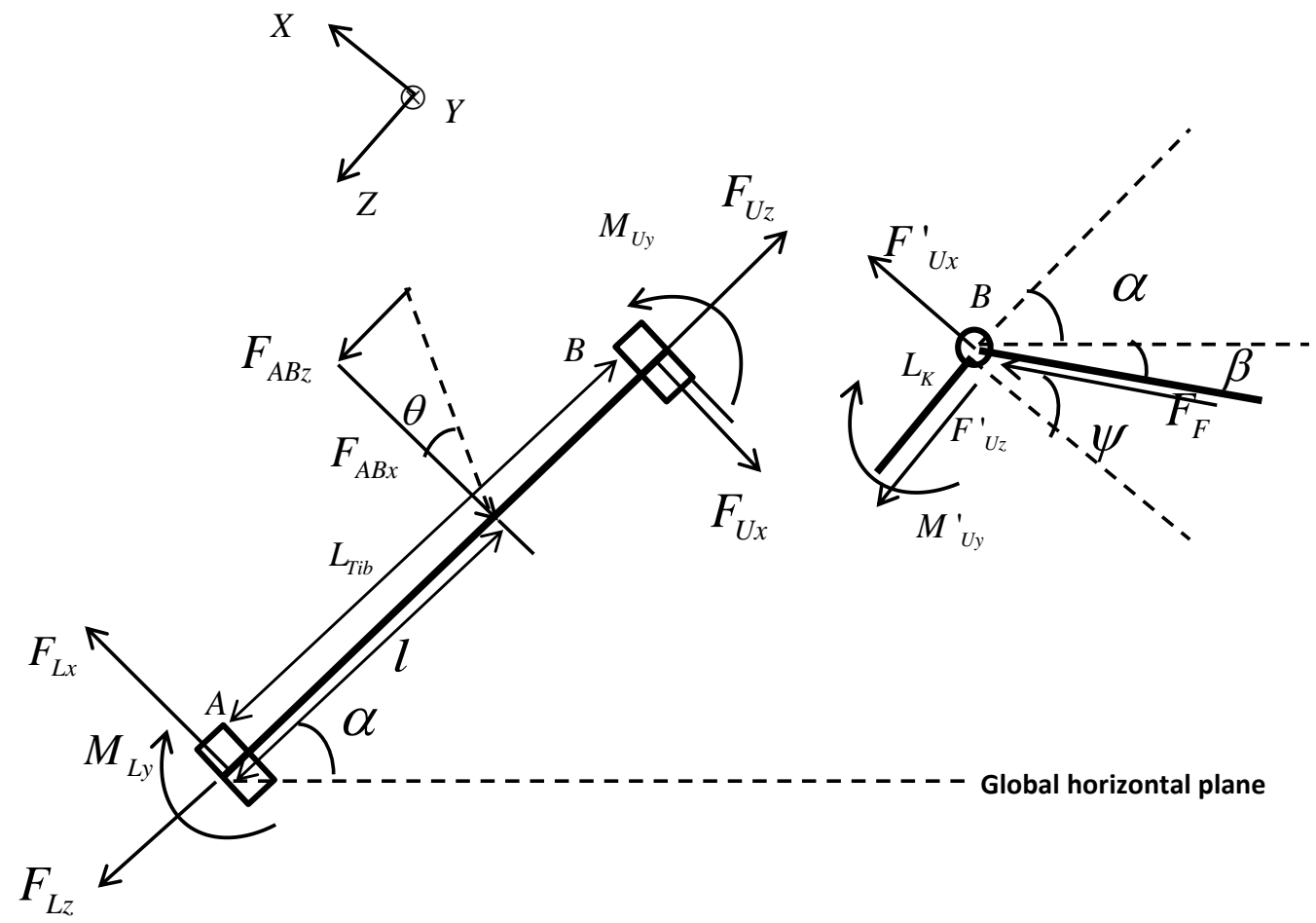

Figure 31. Free body analysis of cases with inflatable knee bolsters

The derived diagram captured the time phase when the inflatable knee bolster deployed and engaged with the tibia region. Obviously, the derived free body diagram was a simplification: the human lower limb is deformable and the whole impact event was essentially a dynamic problem. However, for kinetics analysis, if the tibia was assumed as a rigid beam element, the multi-body model could be served to depict the kinetics under knee airbag deployment.

To apply Newton's second law, the resultant force and moment equations of the tibia could be derived as:

$$
\begin{aligned}
& \sum F_{z}=F_{A B z}+F_{L z}-F_{U z}=m a_{z} \\
& \sum F_{x}=-F_{A B x}+F_{L x}-F_{U x}=m a_{x} \\
& \sum M_{y}=M_{L y}-M_{U y}+F_{U x} L_{T i b}+F_{A B x} l=I \ddot{\alpha}
\end{aligned}
$$

Current instrumentation did not have the capability to measure linear and rotational acceleration; therefore, the functions could not be solved with a definite analytical solution, as the number of unknown variables exceeded the number of equations. Had the Hybrid III dummy been instrumented with accelerometers to measure the linear accelerations $a_{x}, a_{z}$ as well as the rotational acceleration $\ddot{\alpha}$, the quantitative knee airbag force could be derived from the equations above. For the femur region, the corresponding equations could be derived similarly as follows: 


$$
\begin{aligned}
& \sum F_{z}=-F_{U z}^{\prime}+F_{F} \sin \psi=M a_{z} \\
& \sum F_{x}=-F_{U x}^{\prime}+F_{F} \cos \psi=M a_{x} \\
& \sum M_{y B}=F_{U x}^{\prime} L_{K}-M_{U y}^{\prime}-F_{F} \cos \psi L_{K}=I^{\prime} \ddot{\beta}
\end{aligned}
$$

For cases with the standard knee bolster, contact between the knee and the knee bolster occurred at relatively later stage, compared to tests with the IKB. Correspondingly, both the tibia angle and femur angle increased as the occupant further engaged with the bolster panel and leaned forward (Figure 32). The contact occurred at later stage resulted in a negative tibia bending moment MY at proximal and distal tibia load cells, and the corresponding tibia and femur kinematics were different from the case with IKB. The knee bolster force acted upon the anterior knee joint, with decomposed force component $F_{B z}$ and $F_{B x}$ denoted in the free body diagram. As no instrumentation was available for the calculation of knee bolster force, currently figure could only qualitatively demonstrated the kinematics of the case with standard bolster.

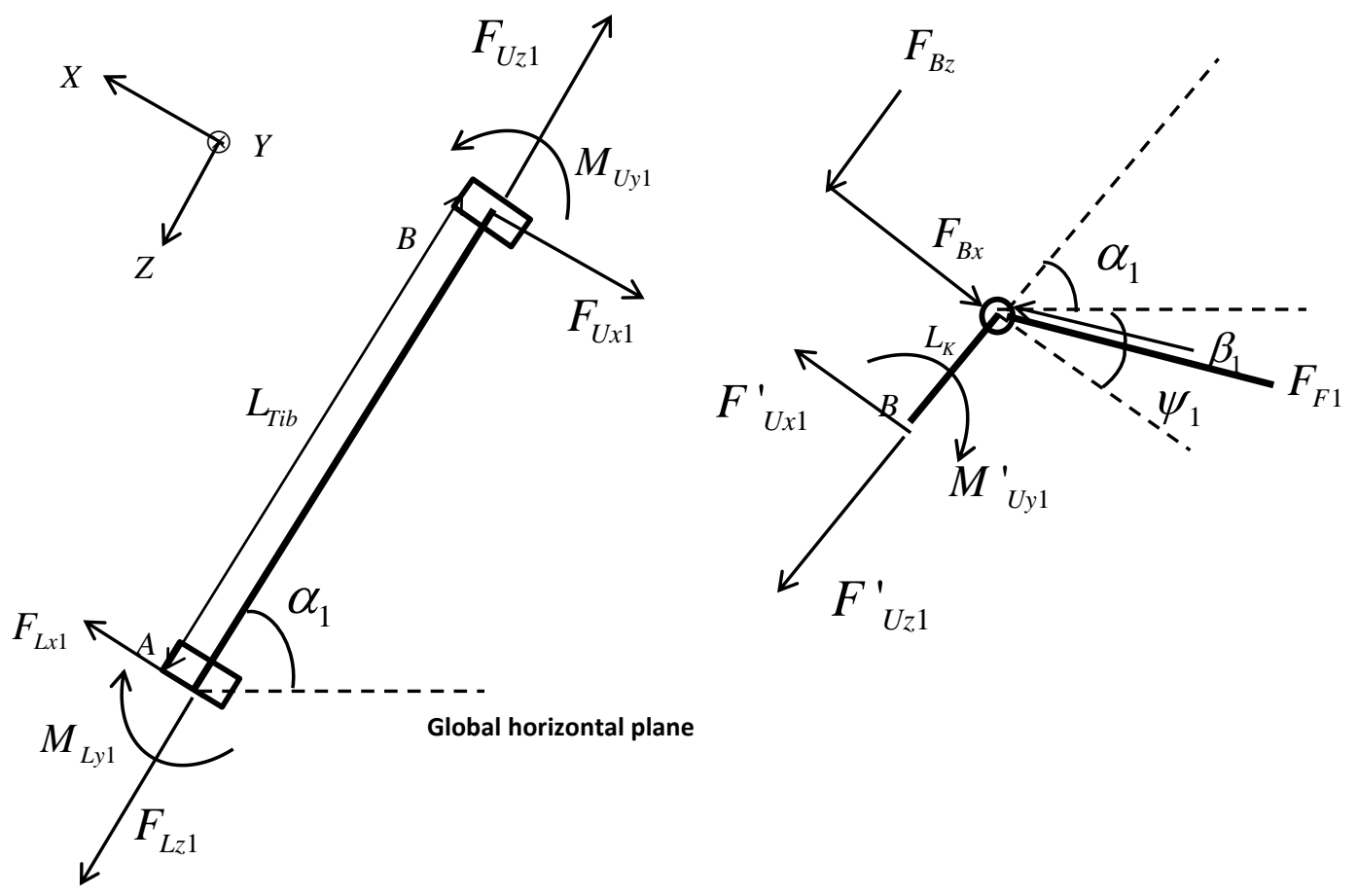

Figure 32. Free body analysis of cases with standard knee bolsters

For the truncated tibia region, the resultant force and moment functions could be derived as:

$$
\begin{aligned}
& \sum F_{z}=F_{L z 1}-F_{U z 1}=m a_{z 1} \\
& \sum F_{x}=F_{L x 1}-F_{U x 1}=m a_{x 1} \\
& \sum M_{y A}=M_{L y 1}-M_{U y 1}+F_{U x 1} L_{T i b}=I \ddot{\alpha}_{1}
\end{aligned}
$$

For the femur region, the equations could be written as: 


$$
\begin{aligned}
& \sum F_{z}=F_{U z 1}^{\prime}+F_{B z}+F_{F 1} \sin \psi_{1}=M a_{z 1} \\
& \sum F_{x}=F_{U x 1}^{\prime}-F_{B x}+F_{F 1} \cos \psi_{1}=M a_{x 1} \\
& \sum M_{y B}=M_{U y 1}^{\prime}+F_{B x} L_{K}-F_{F 1} \cos \psi_{1} L_{K}=I^{\prime} \ddot{\beta}_{1}(16)
\end{aligned}
$$

Referring to the derived free body diagram, the following analysis aims to investigate the moment and force change as a function of dummy lower limb orientation. The analysis combined the kinematics information (tibia moment, tibia force) and the kinetics information (tibia angle), and provided a direct way to cross-compare any difference of the dummy lower extremity response from the IKB and standard knee bolster. Additionally, this approach could indicate the change in driving posture induced by the deployment of the IKB, as well as to illustrate the compressive force and bending moment effect on the change of tibia index in two different test conditions.

Coordinates were measured from the film analysis using MEMRECAM GXLink (Nac Image Technology, Simi Valley, CA), and trigonometric functions were used to calculate the tibia angle and femur angle from the highspeed camera films. The tibia angle trace was collected at each video frame, corresponding to the time range of $18 \mathrm{~ms}$ (i.e., IKB and DAB firing time) to $80 \mathrm{~ms}$, with a time increment of $1 \mathrm{~ms}$. Since the camera recording frequency was set at 1 frame/ms, while test data was collected at every $0.05 \mathrm{~ms}$, tibia angles were marked the same values as the closet neighboring time step when peak values of tibia force and moment occur within $1 \mathrm{~ms}$ of time interval, in order to retain the integrity of test information.

FMVSS test 779 with standard bolster and test 862 with IKB were selected as examples for comparison, and corresponding legends denoted the peak range for tests in the same condition (Figure 33). Initial driving posture was similar for both tests, with left tibia angle of $45^{\circ}$ for test 779 and $46.27^{\circ}$ for test 862 , respectively. Curves from two tests started to diverge after firing of airbag at $18 \mathrm{~ms}$, indicating the reduction of tibia angle due to the deployment of IKB. This deployment also resulted in the positive peaks in tibia bending moment MY, as evidenced by the spike in both upper and lower tibia load cells. For test 862 with IKB, left tibia angle decreased from initial posture to $33.4^{\circ}$, which mapped with the interaction of IKB from $18 \mathrm{~ms}$ to $52 \mathrm{msec}$. The change of posture and reduction of tibia angle was attributed to the deploying IKB. At later stage, due to the change in sled impulse signal and the change of location of deployed IKB acting force on the tibia, bending moment MY changed direction at both proximal and distal tibia, which led to the increase in tibia angle at ensuing stage. For test with standard bolster, the tibia angle sustained a monotonic increase with the concomitant increase in tibia compression force and bending moment. 

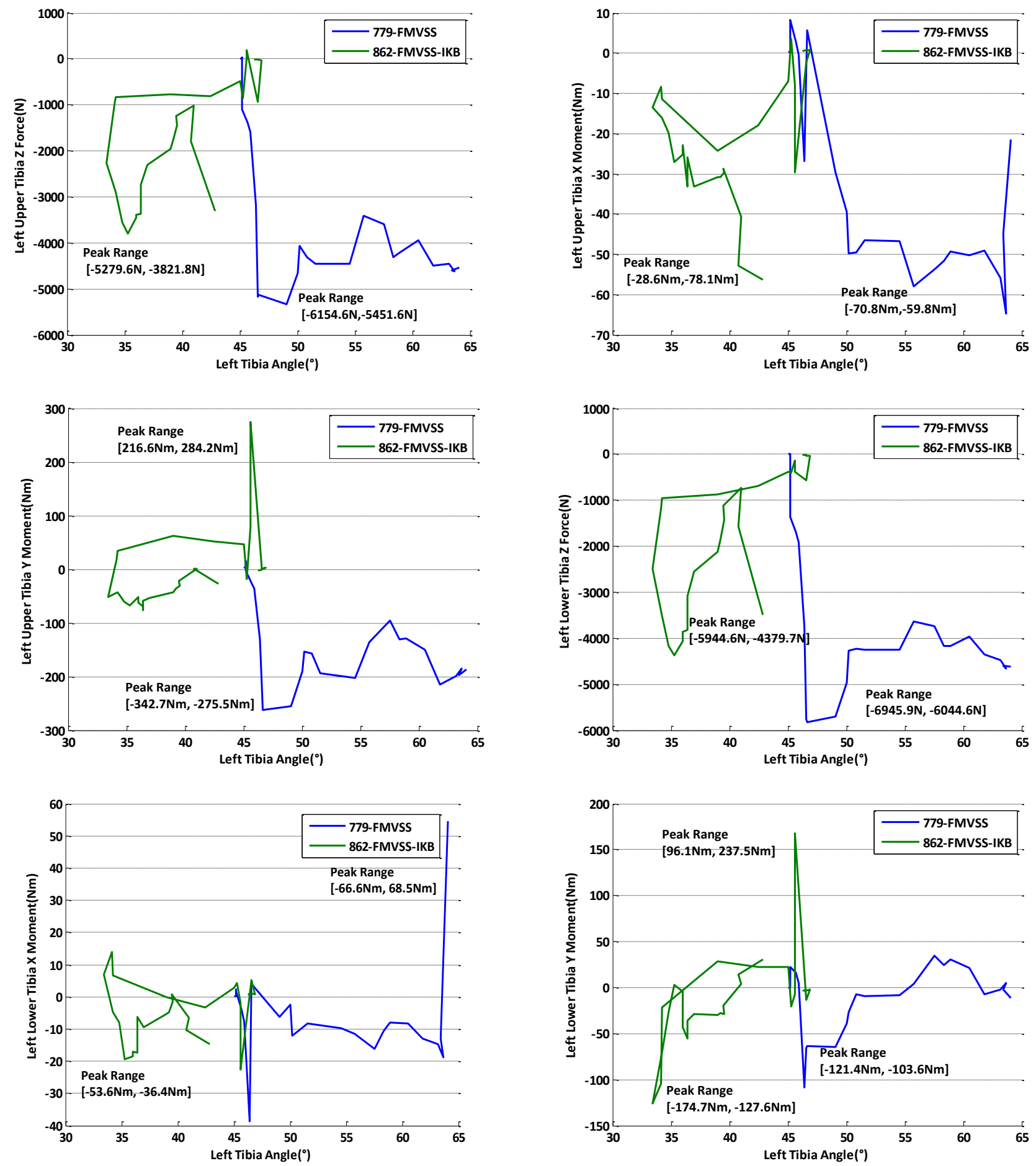

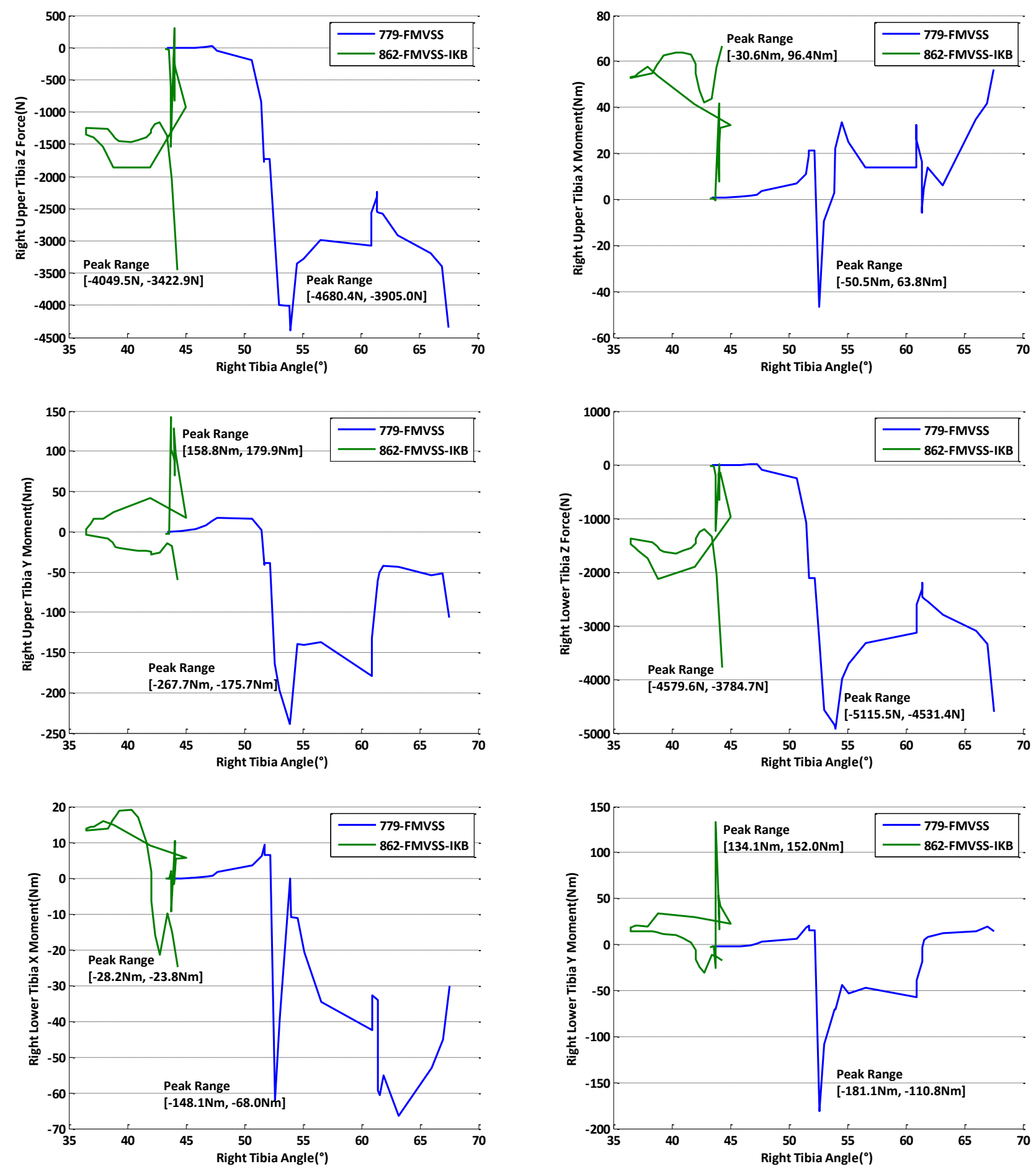

Figure 33. Comparison of tibia responses as a function of tibia angle for FMVSS208 tests

For test with inflatable knee bolster, the tibia index was predominantly affected by the resultant tibia bending moment, and more specifically, the tibia bending moment MY component. Tibia index reached peak value of 1.23 at $27.9 \mathrm{~ms}$, which is the same time with the occurrence of peak tibia bending moment MY (Figure 34). Similarly, the left lower tibia reached a peak value of 1.06. The tibia index then decayed down with the decrease of tibia angle. For test with standard knee bolster, tibia index reached maximum value of 0.71 and 0.72 for left upper tibia and left lower tibia at $60.4 \mathrm{~ms}$ and $58.1 \mathrm{~ms}$, respectively, which is much lower 
relative to the IKB test. The right upper tibia index was comparable between the IKB test 862 and the bolster test 779, while the right lower tibia was lower in IKB test (0.60) than in bolster test (1.04).
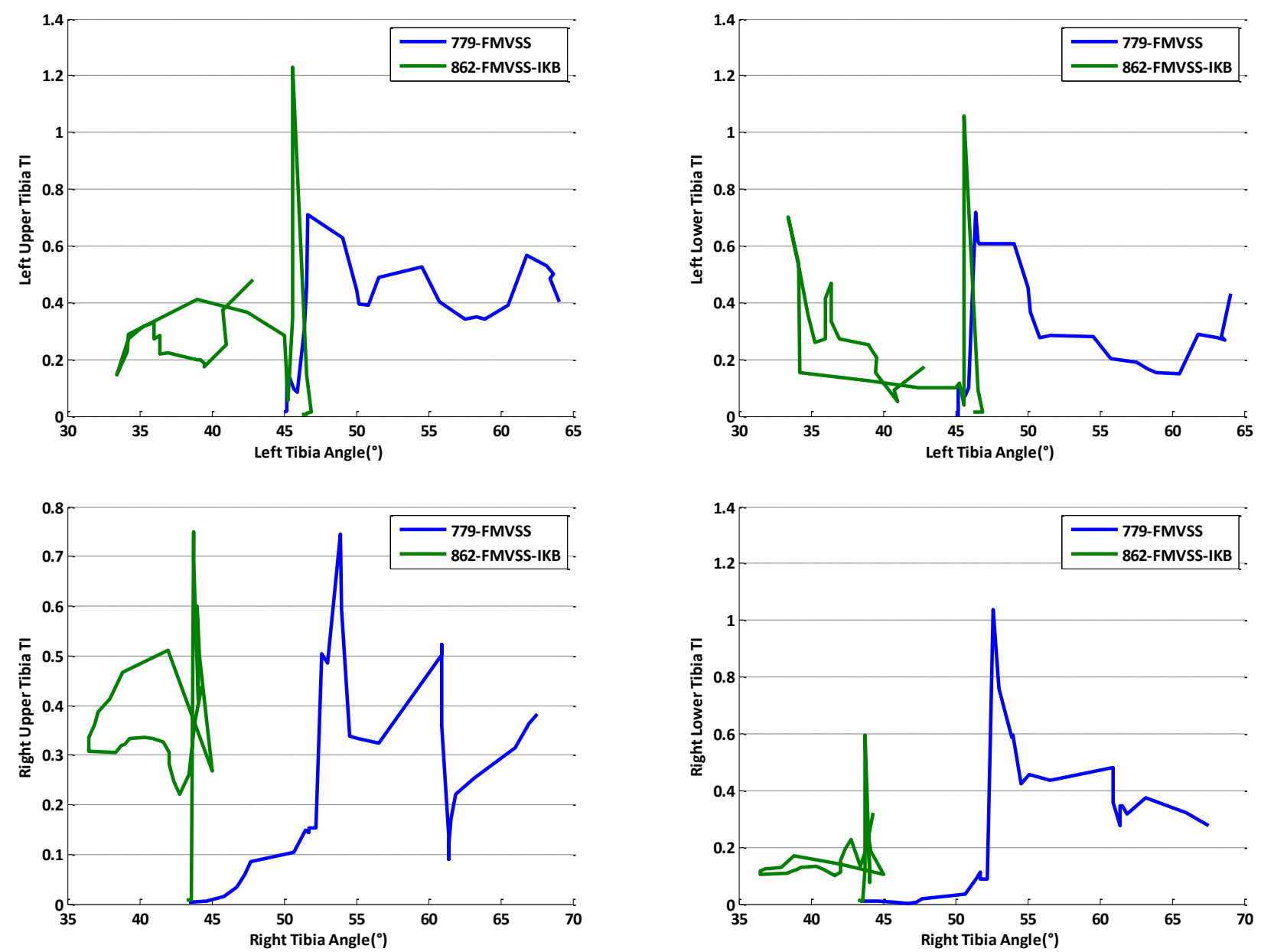

Figure 34. Comparison of tibia indices as a function of tibia angle for FMVSS208 tests

NCAP test 794 with standard bolster and test 801 with IKB were selected for comparison of tibia responses (Figure 35). For test with standard knee bolster, left tibia angle monotonically increased from initial angle of $45.5^{\circ}$ at $18 \mathrm{~ms}$ to final angle of $57.5^{\circ}$ at $80 \mathrm{msec}$. Correspondingly, for test with IKB, left tibia angle decreased from initial angle of $47.0^{\circ}$ to $34.9^{\circ}$ during the deployment of IKB, and then increased from $34.9^{\circ}$ to $44.5^{\circ}$ as the belted dummy engaged forward towards the instrument panel. Similar to the findings from FMVSS tests, a positive tibia bending $\mathrm{Y}$ moment was the predominant factor for the reduction of tibia angle during the deployment of the inflatable knee bolster. Additionally, the left tibia compression force reached peak magnitude at a tibia angle of $35^{\circ}$ with IKB present, compared to $49^{\circ}$ where peak occurred with standard bolster. Right tibia angle showed a change similar to the left aspect: a monotonic increase from $46.8^{\circ}$ to $57.8^{\circ}$ for test 797 , and a decrease from $46.8^{\circ}$ to $40.0^{\circ}$ accompanied by ensuing increase up to $50.9^{\circ}$ for test 801 . 

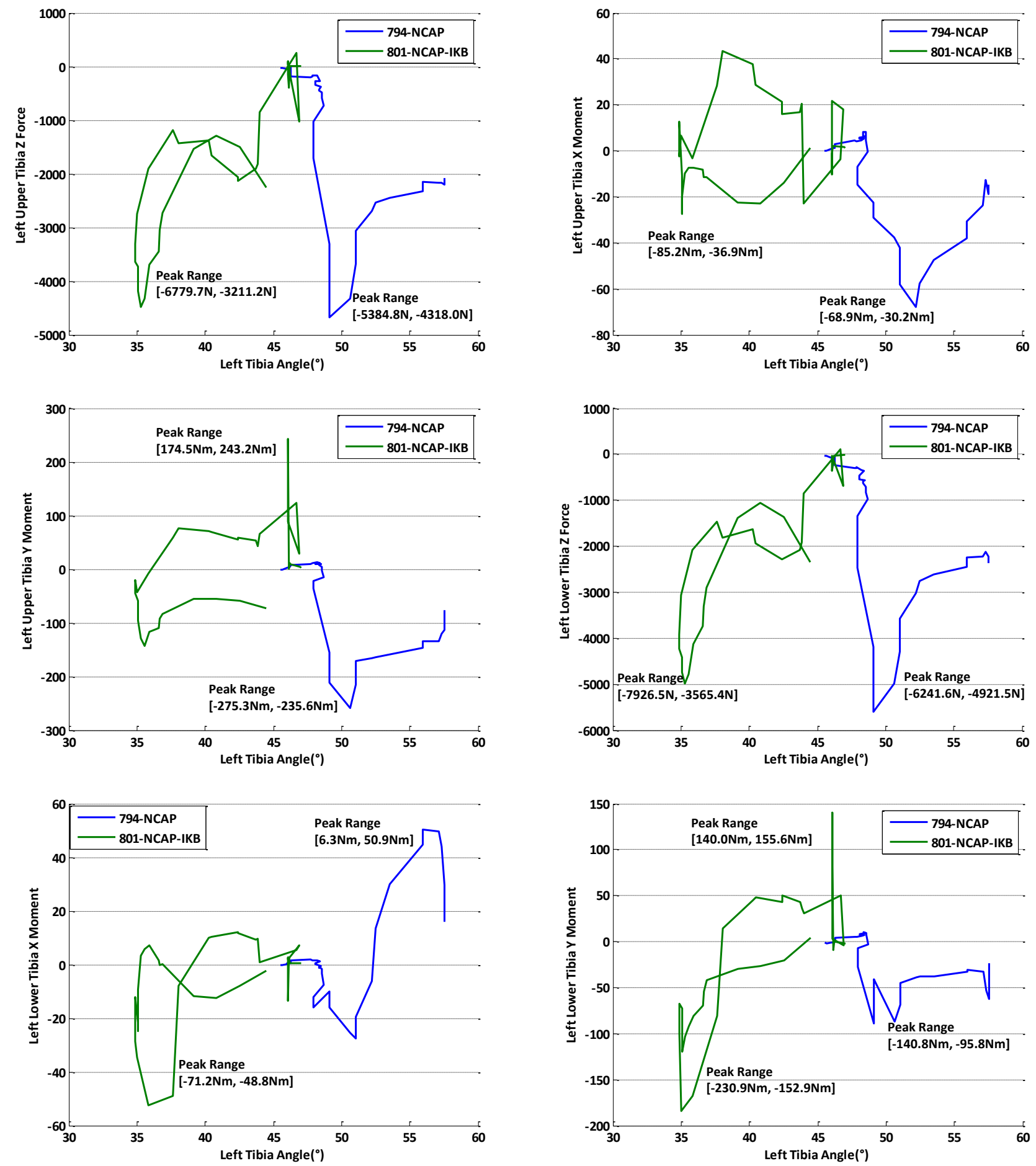

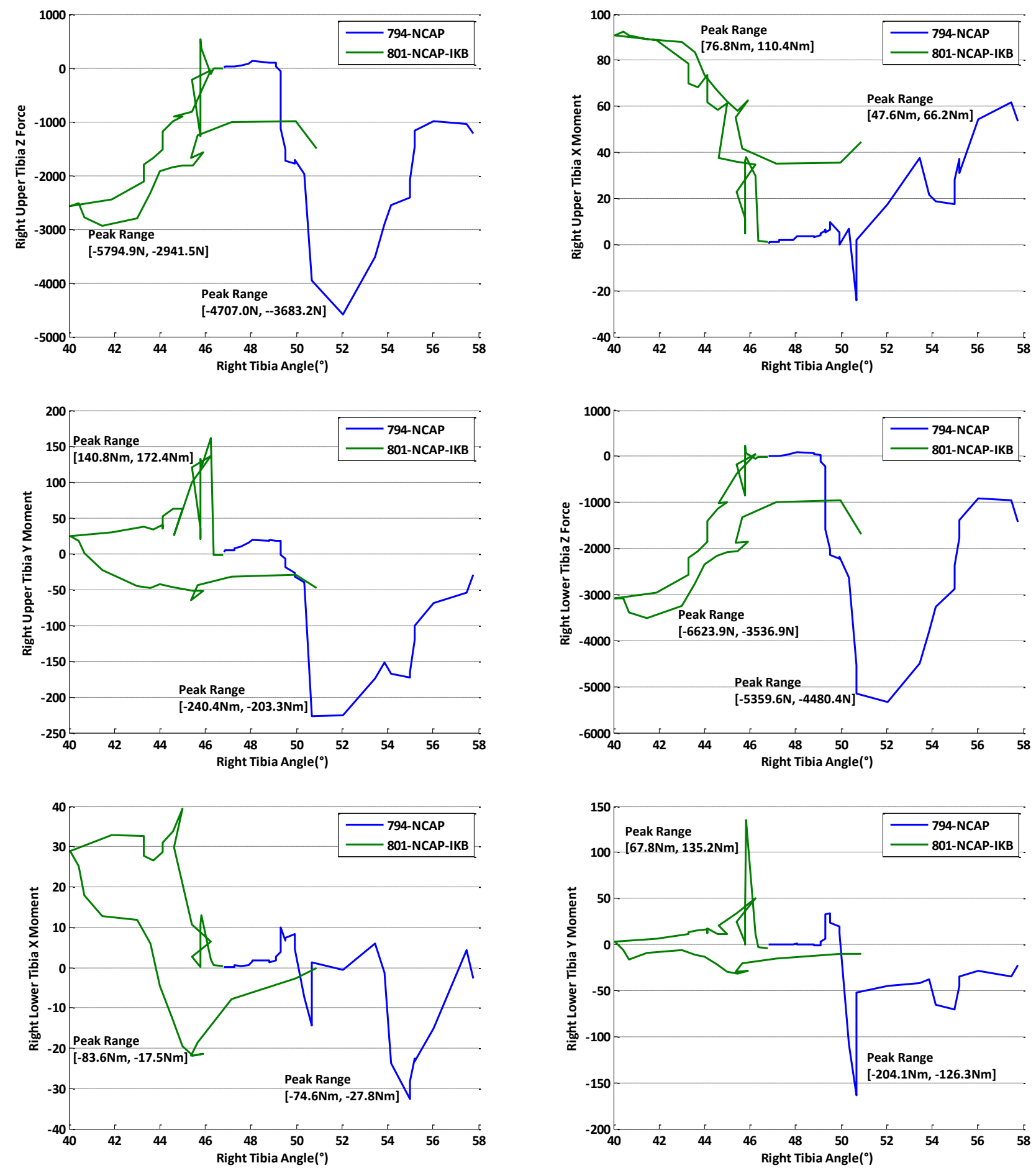

Figure 35. Comparison of tibia responses as a function of tibia angle for NCAP tests

For NCAP test 794 with standard bolster, tibia index peaked at around 57 ms with a left upper tibia index of 0.76 , and a left lower tibia index of 0.68 (Figure 36). Contrarily, for test 801 with IKB, peak tibia index of left upper tibia occurred during the deployment of the IKB, with a value 1.10 at $27 \mathrm{~ms}$. Left lower tibia, however, did achieve a peak value of 1.10 at later stage due to the change of tibia bending moment direction. Right upper tibia sustained a peak tibia index of 0.63 for bolster test at $56 \mathrm{~ms}$ and a peak value of 0.77 for IKB test at $25 \mathrm{~ms}$. The right lower tibia index was lower for IKB test (0.60) than the standard bolster test (1.03). 

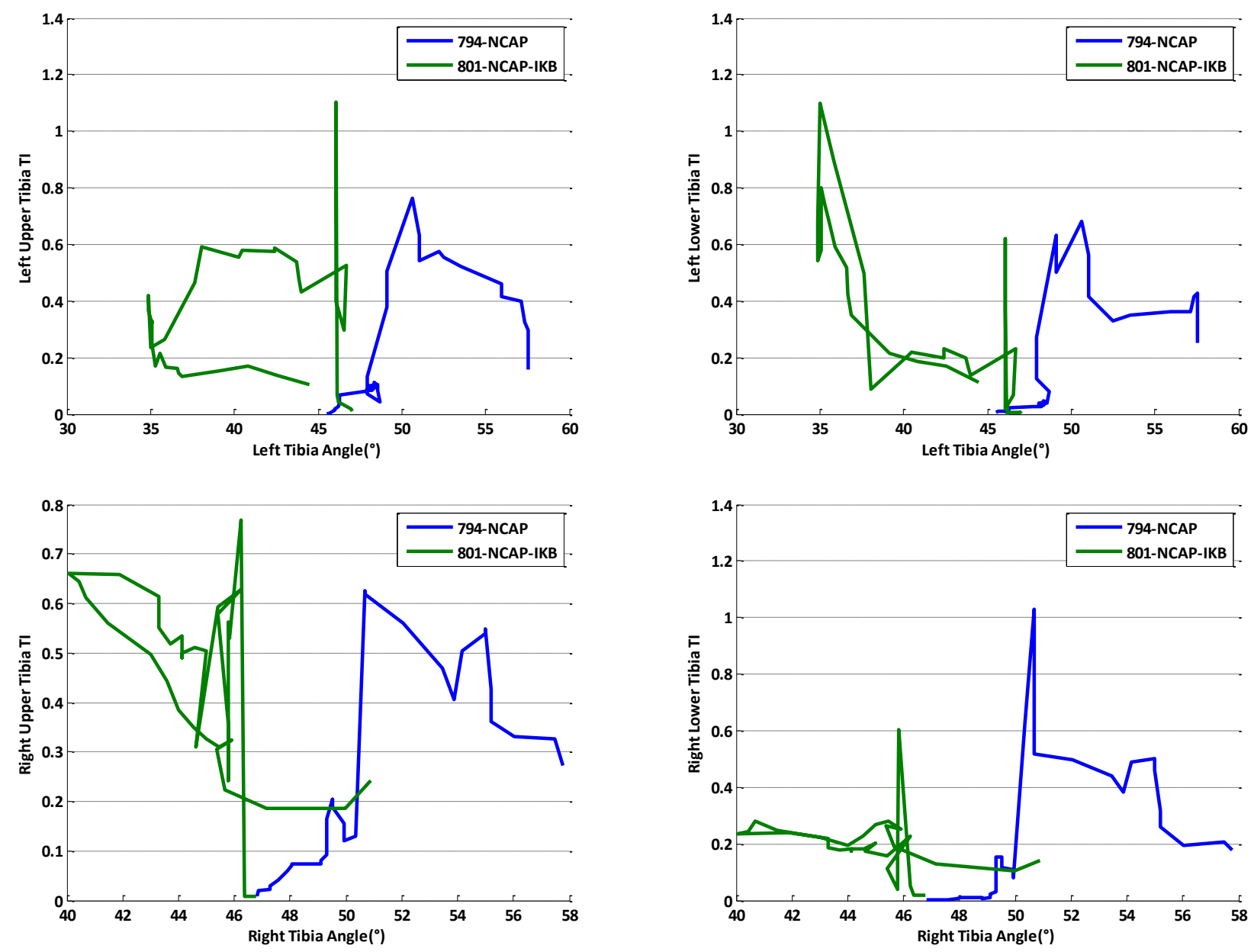

Figure 36. Comparison of tibia indices as a function of tibia angle for NCAP tests

\section{Injury Risk Function}

For FMVSS 208 test, results from the injury risk functions predicted an increased injury risk of AIS2+ leg shaft fractures, especially for upper left leg shaft under IKB deployment (Figure 37). For test 862 with IKB, the corresponding leg shaft fracture injury risk peaked to $51.2 \%$, which is much higher than the baseline tests with the conventional knee bolster. The differences in AIS2+ tibial plateau or condyle injury risk between tests with standard bolster and with IKB were less distinct. 


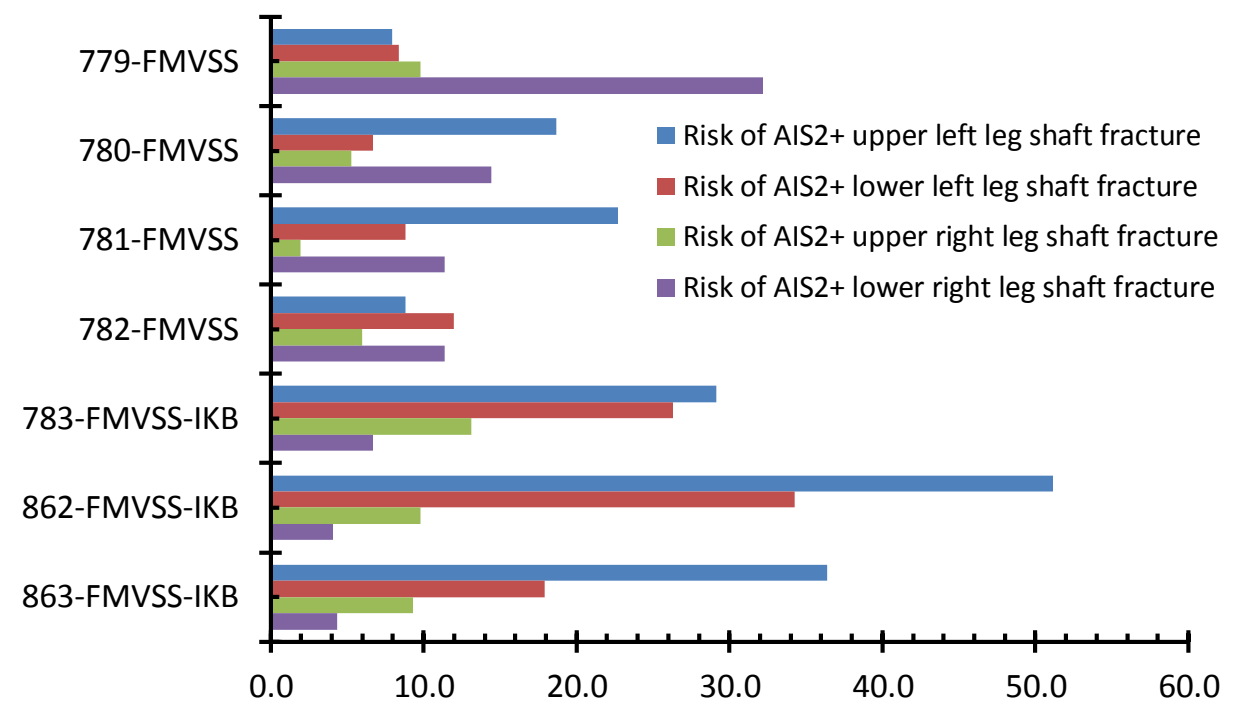

Figure 37. Risk of AIS2+ left and right leg shaft fractures as a function of tibia index for FMVSS tests (\%)

For NCAP tests, results from the injury risk functions indicated a marginal increase of injury risk in AIS 2+ leg shaft fractures with IKB (Figure 38). Specifically, the left upper tibia, left lower tibia, and right upper tibia fractures sustained an increased injury risk, which was positively connected with the increase in tibia indices. Left lower tibia achieved the highest injury risk for NCAP tests with IKB, with an increase of $25.0 \%$ in injury risk compared to standard knee bolsters.

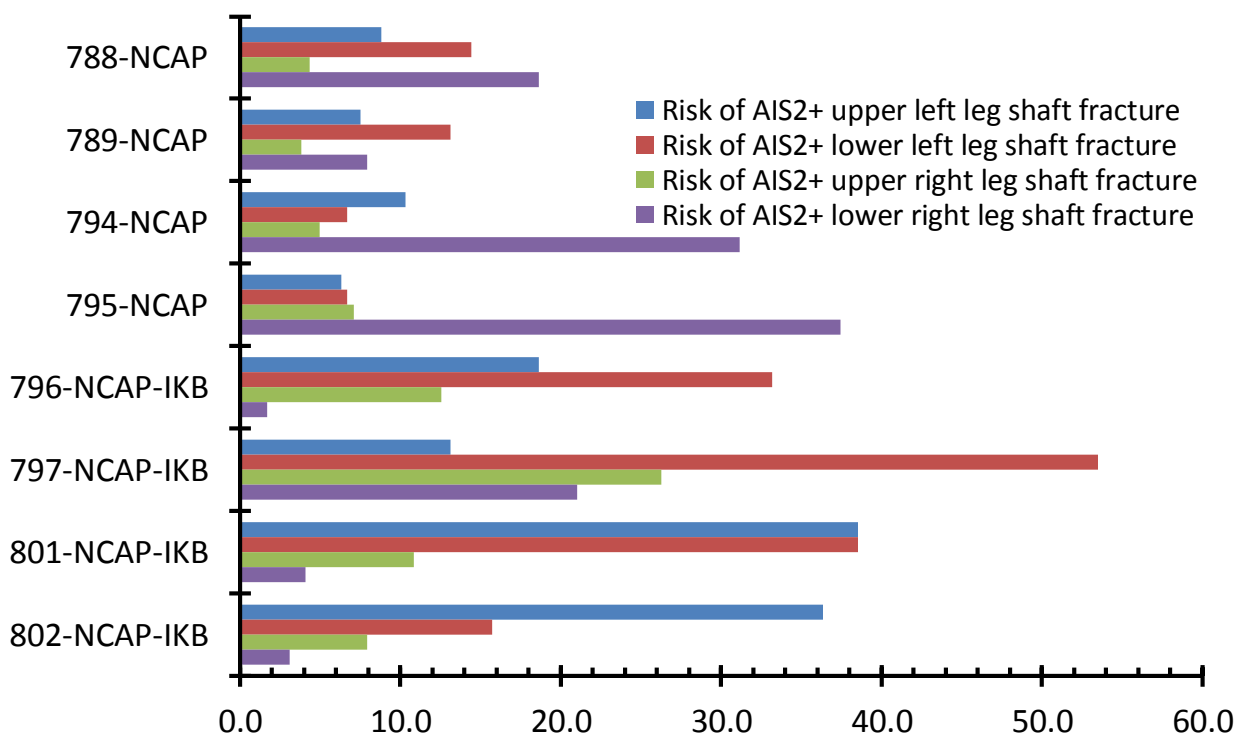

Figure 38. Risk of AIS2+ left and right leg shaft fractures as a function of tibia index for NCAP tests (\%) 


\subsection{Comparison of Response for FMVSS and NCAP tests \\ FMVSS Tests}

To examine the potential difference of tibia and femur force resulted from the knee bolster types, test 779 (standard knee bolster) and test 783 (IKB) were selected for direct comparison (Figure 39-40). The reason to compare the response of tibia force with respect to femur force was to investigate any loading path difference between the two test conditions. Tibia forces were truncated at peak magnitude and plotted against the corresponding femur force. As indicated from the plots, for test with standard knee bolster, femur force and tibia force were mostly positive correlated. Monotonic increase was observed in the left tibia force from starting time to approximately $60 \mathrm{~ms}$, in conjunction with the accumulation of femur force. For tests with the inflatable knee bolster, the gradient of curve had a slower increment rate until $30 \mathrm{~ms}$, which reflected the early contact between femur and the deploying IKB with reduced contact stiffness. Little distinction was noticed for peak magnitude of left upper tibia and left lower tibia force FZ between these two tests, but the

tibia force had a higher increment at later stage for IKB tests. This could be possibly explained to the change of lower limb posture resulted from the IKB, as IKB decreased the tibia angle and femur angle during its deployment, and given the same amount of deceleration from the toe pan, higher loading was transmitted from the decomposition force projected along the tibia direction. Right tibia sustained significant more oscillation in test with the IKB, which was resulted from the change in foot placement. Initial posture of the right foot was placed on the accelerator pedal, and deployment of the IKB led to the foot sliding forward and heel stomped on the floor pan. The first peak in the right tibia force FZ was attributed to the compression of IKB deployment against the tibia shaft and plateau region. After the deployment of the IKB, the IKB deflated and contact occurred between the IKB plastic panel surface and the tibia plateau region. At this point of time, due to the forward excursion of the unbelted dummy, the tibia angle increased again, and resulted in a second peak of the tibia force $F Z$ from the deceleration force of the toe pan region. The second peak of tibia compression force occurred at approximately $80 \mathrm{~ms}$ with dummy further engaged with the bolster panel. 


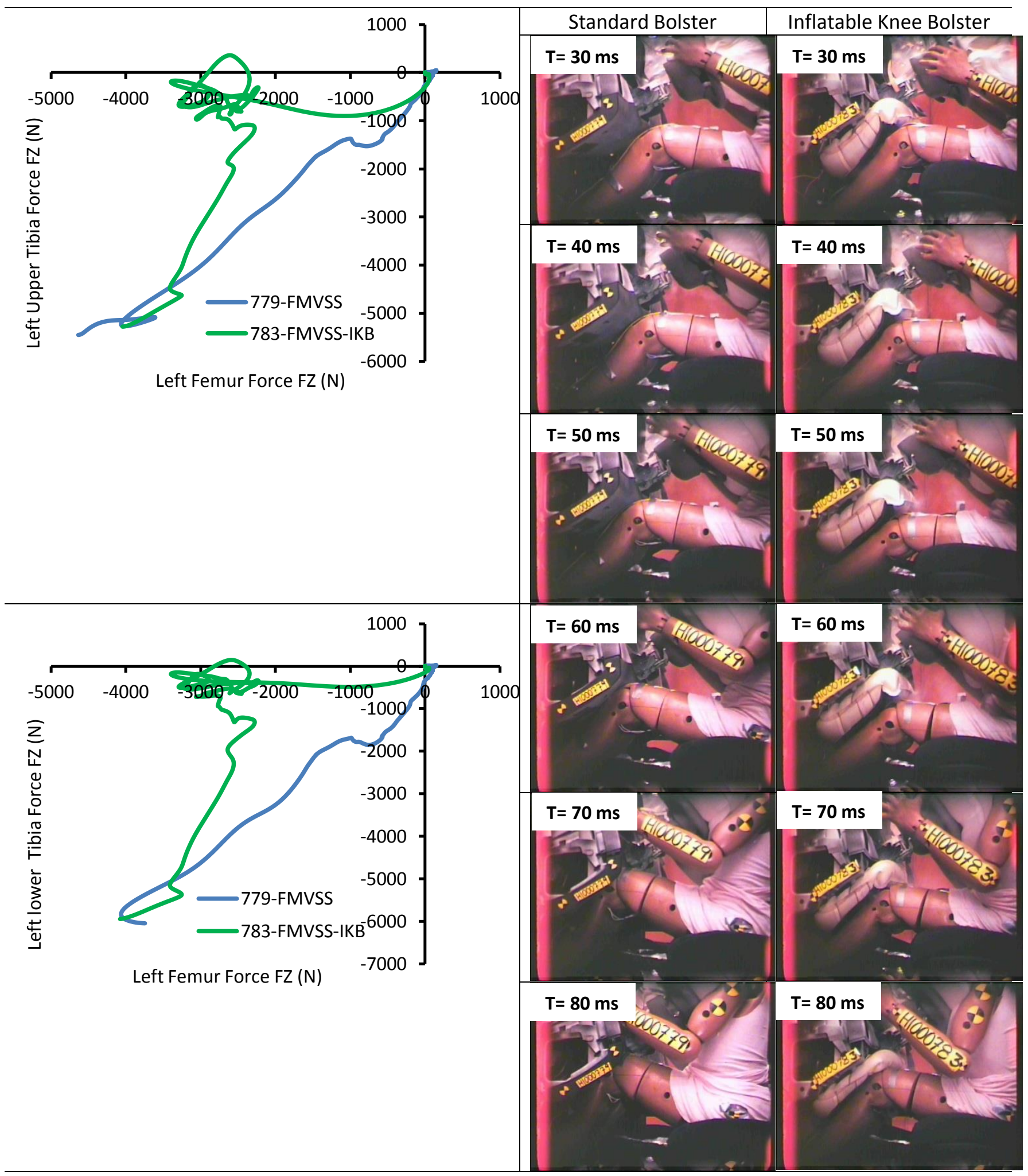

Figure 39. Comparison of left tibia force for two representative FMVSS tests 


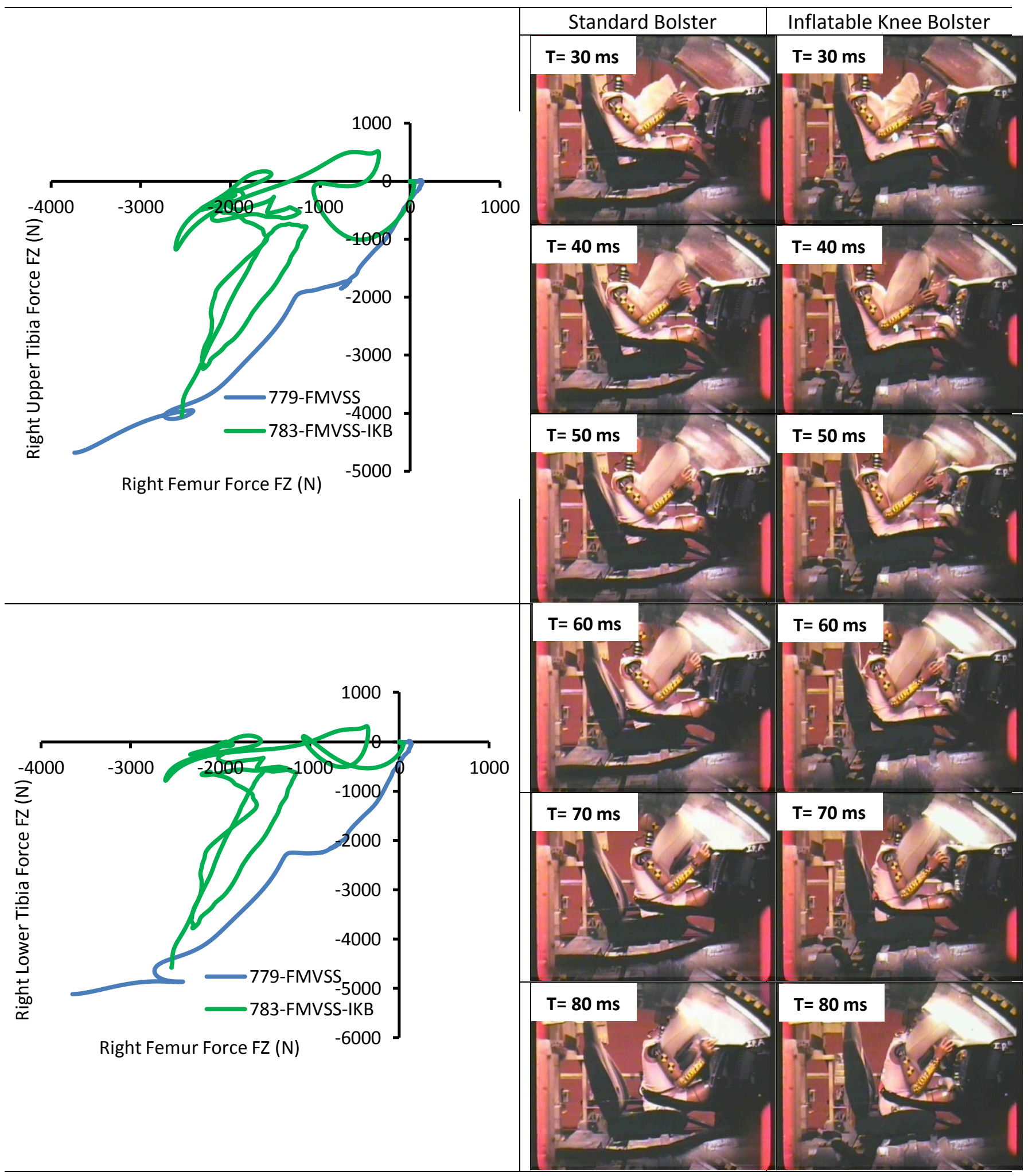

Figure 40. Comparison of right tibia force for two representative FMVSS tests 


\section{NCAP Tests}

Two representative NCAP tests, namely test 788 with the standard bolster and test 797 with IKB, were selected for direction comparison (Figure 41-42). Test with IKB resulted in higher compressive loading in left and right, lower and upper tibia in this case. IKB deployment provided early engagement with the lower limb, and decreased the tibia and femur angle during the interaction with the bolter. Left upper tibia force reached peak magnitude of $-6779.7 \mathrm{~N}$ at $55.7 \mathrm{~ms}$ in test 797 with IKB, and peak magnitude of $-5384.8 \mathrm{~N}$ at $56.1 \mathrm{~ms}$ in test 788 with the standard knee bolster. The two different slopes in the data curve for IKB tests corresponded to two different stages. First stage referred to the early engagement with the IKB, with the increase in femur force. Second stage referred to the further engagement of the tibia with the deployed IKB. Similar with the FMVSS tests, with the presence of IKB, tibia compression force also elevated faster in this stage, as the lower limb was positioned in a more extended posture for IKB compared to conventional bolster. The left lower tibia force FZ was also higher for test with IKB $(-7926.5 \mathrm{~N}$ at $55.6 \mathrm{~ms})$ than test with standard knee bolster $(-6241.6$ $\mathrm{N}$ at $56.4 \mathrm{~ms}$ ). Entrapment occurred for the right tibia for test 788 with standard bolster, which resulted in further concentrated loading. Test with IKB prevented the entrapment occurrence, and the loading from the toe pan transmitted from the floor up towards the tibia region. This effect finally resulted in a higher loading at both right upper and right lower tibia in the IKB test.

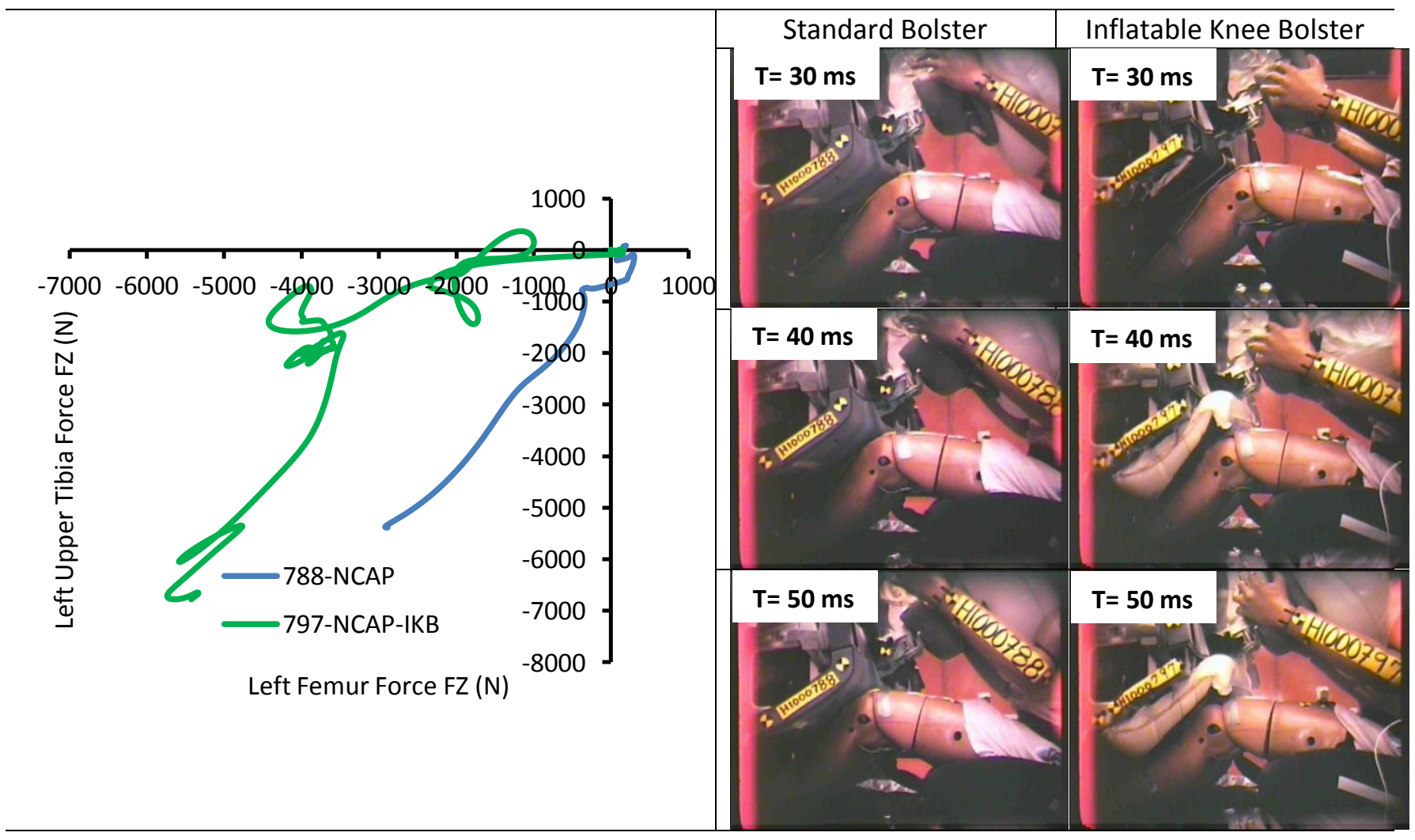




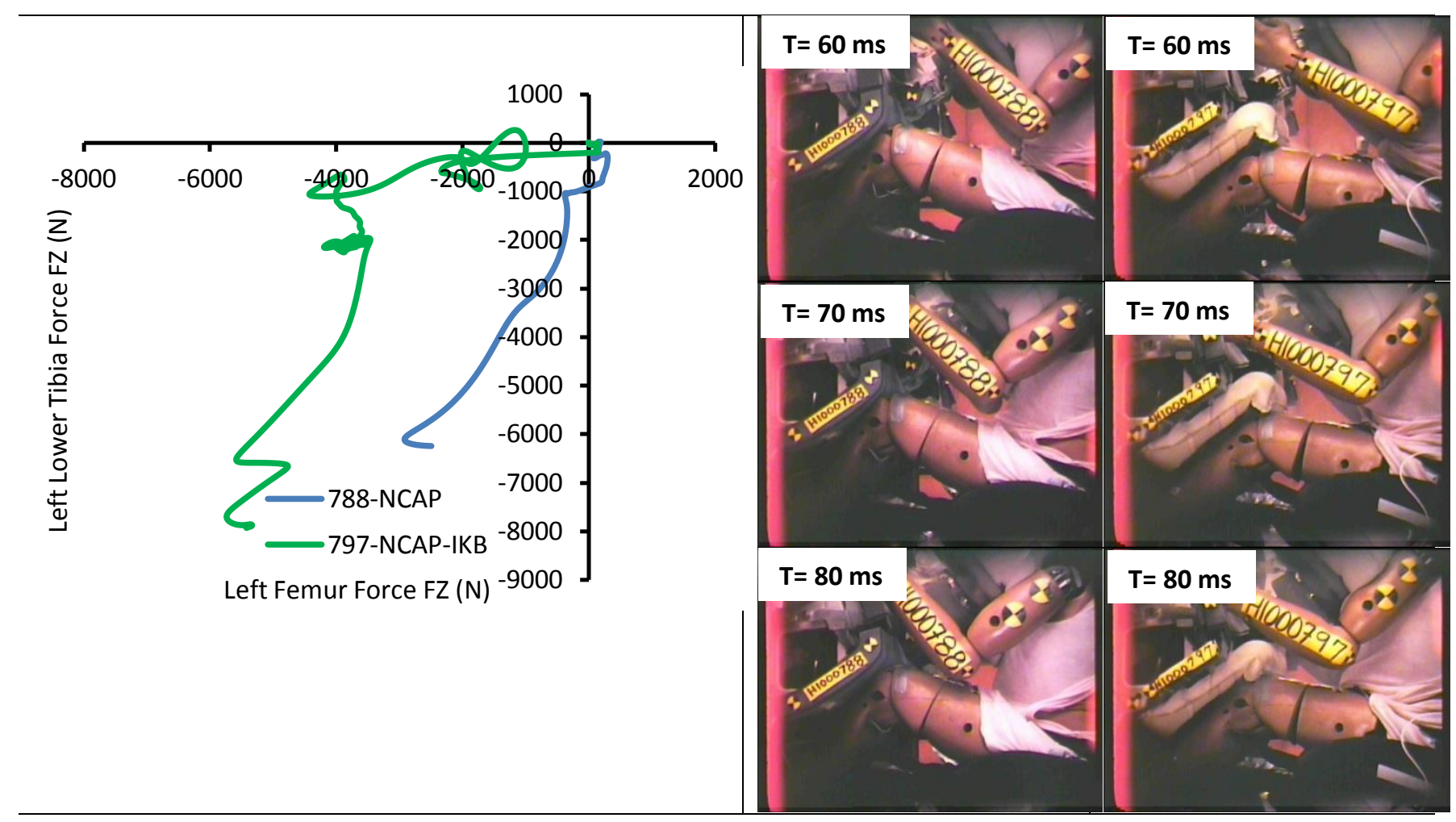

Figure 41. Comparison of left tibia force for two representative NCAP tests

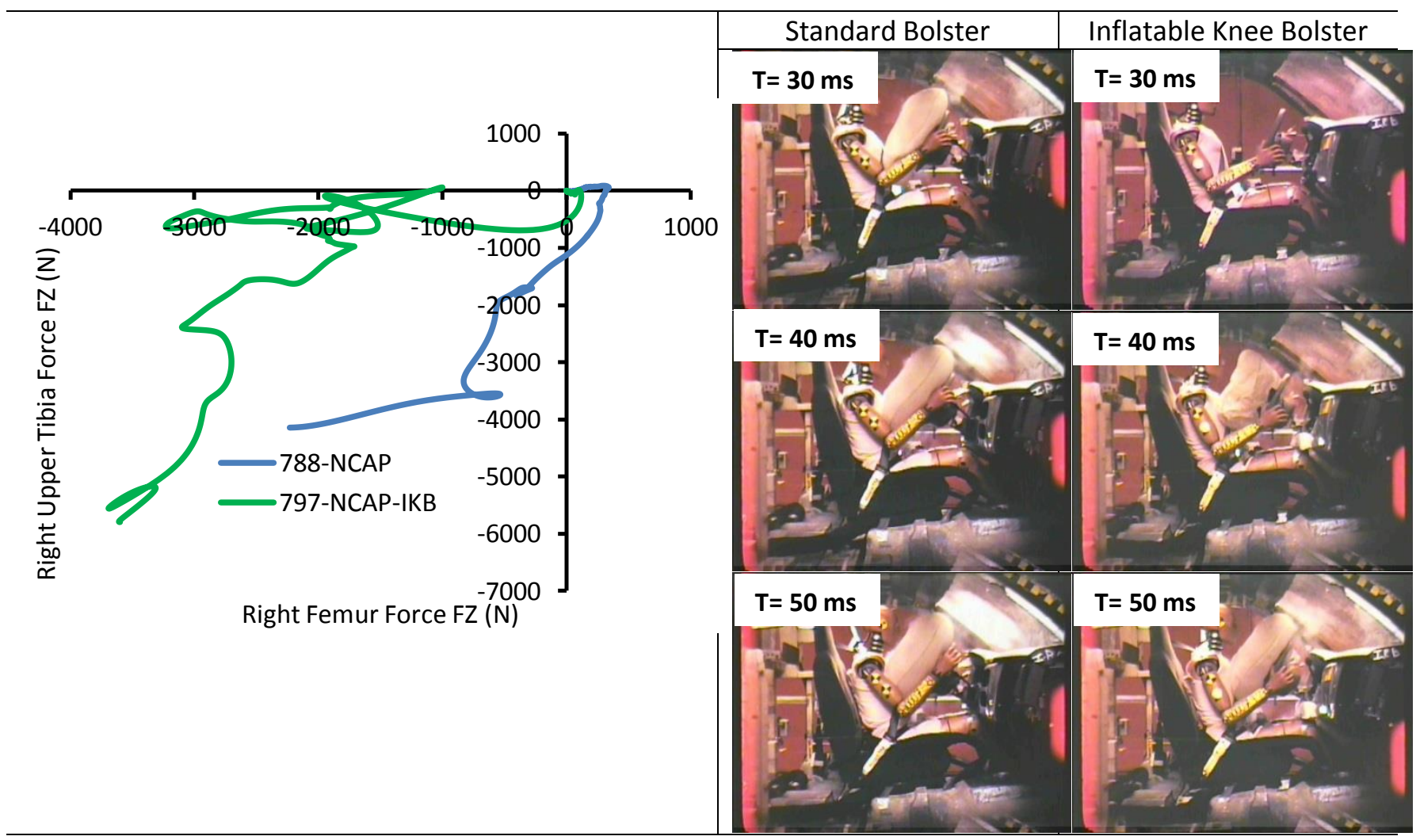




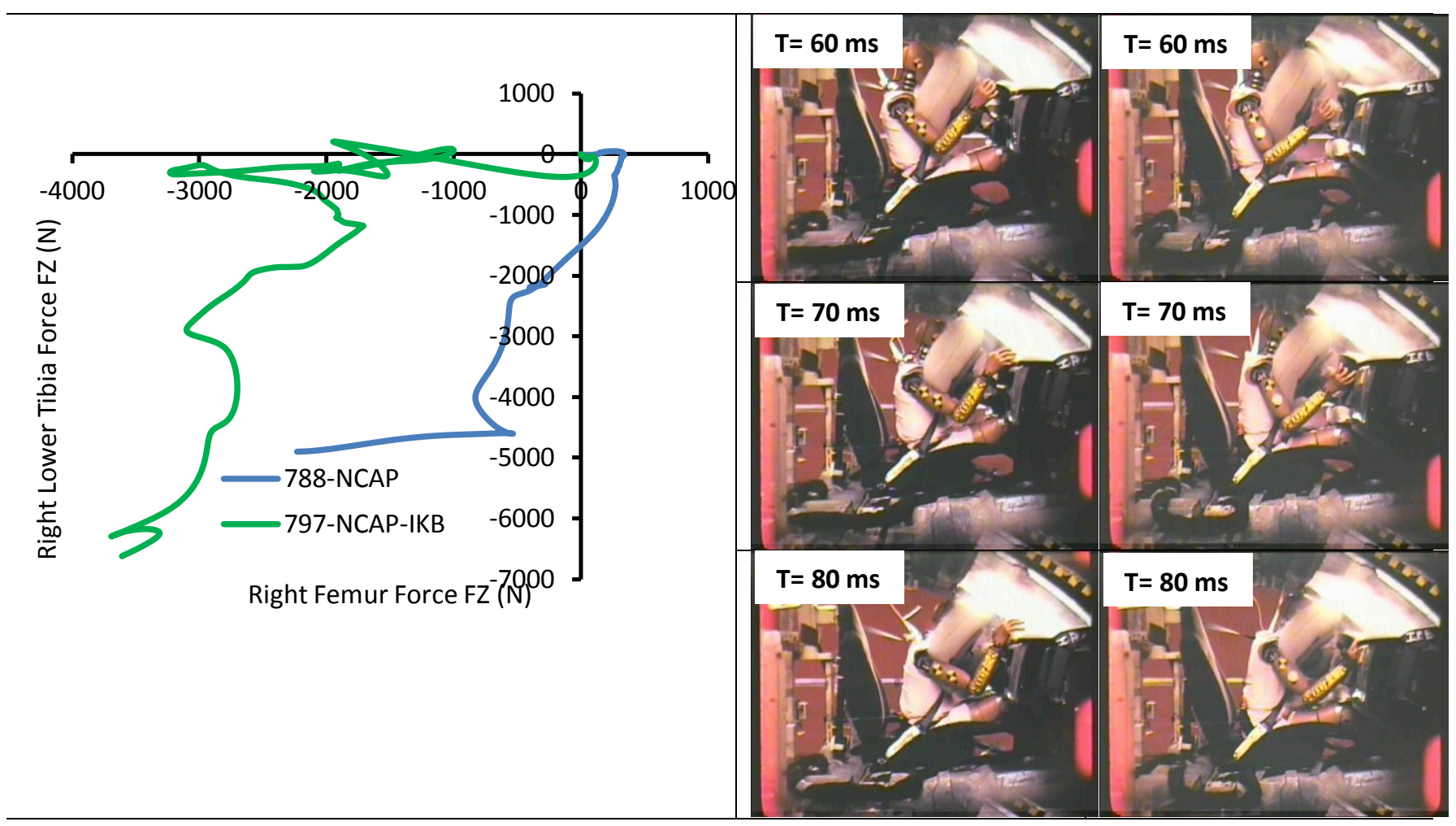

Figure 42. Comparison of right tibia force for two representative NCAP tests

\subsection{Discussion}

For FMVSS 208 tests, since the ATD was not constrained with shoulder belt and lap belt, relatively large amounts of pelvic forward motion and upper torso excursion were observed. IKB provided early engagement with the lower limb, but resulted in a positive tibia bending moment MY at proximal and distal tibia during the deployment stage as the IKB deployed against at tibia mid-shaft region, which was the dominant factor in the tibia index calculation. The alternation in tibia moment direction was attributed to the change in the location of the contact region, as the tibia plateau and knee engaged with the deflated IKB later on, as the legs slid forward due to dummy inertia. Additionally, the sled pulse reached peak at the same time and started to decay, which contributed to the driver posture change with an increase in tibia angle. Consequently, the ensuing posture could potentially lead to an increase in the tibia loading, resulted from the axial component derived from toe pan and foot panel deceleration. Although current tests were performed with a production IKB in a buck test structure typical of a mid-size sedan profile, which represented a fair amount of real-world cases with knee airbag, the effect of altering the lower extremity kinematics was partially dependent on the specific IKB or knee airbag design. In current study, the IKB deployed directly towards the anterior tibia shaft and plateau region: this relatively simple deployment pattern attributed to the initial change in moment and kinematics in the Hybrid-III dummy lower limb. Additionally, fair amount of oscillation was observed in tibia compression force $\mathrm{FZ}$ in both aspects of the lower limb with the IKB presence, 
which was resulted from the reduction of bolster panel stiffness and the deflation of the IKB after deployment, while the anterior tibia region engaged with the IKB surface panel at the same time.

Lateral knee sliding was also observed for some of the IKB tests, causing the abduction of the legs during the IKB deployment, which corresponding to the oscillation in tibia bending moment MX. Femur loadings, however, decreased due to the reduction of bolster stiffness at early contact, which was consistent with the purported design of knee airbags to provide early restraint for the pelvis and lower torso. However, increased tibia index at left upper tibia, left lower tibia and right upper tibia indicated a higher injury risk of tibia and fibula region under the deployment of an inflatable knee bolster for unbelted drivers.

For NCAP tests, seatbelt and retractor coupled with the additional constraint of the IKB provided better control of the upper torso movement. The left femur loadings, however, slightly increased in test 797 and test 801. For test 797, due to late IKB deployment, the femur was at much closer proximity to the IKB during deployment, which resulted in a high axial loading transmitted to the femur region. For test 801 , the left leg of the dummy sustained a valgus bending during the engagement with the deploying IKB due to initial positioning variation, which resulted in an asymmetric loading of the femurs. Not much decrease was observed in the tibia loadings, and the average compression force of the upper and lower, left and right tibia were comparable for tests with standard bolster and IKB. However, the altered and increased tibia bending moment was the predominant factor for the increase in tibia index for IKB tests. Seatbelt use has shown an increasing trend since 1995, and reached $87 \%$ by the year 2014 based on probability-based observation data in the United States (Pickrell et al., 2015). However, this study found that even with the presence of the seatbelt, the lower extremity injury outcome with knee airbag deployment did not change, as results from the belted NCAP tests showed an increasing trend in tibia index with IKB, which was consistent with the unbelted FMVSS 208 tests. The increased injury risk of tibia/fibula and foot/ankle region with the IKB deployment from this study was consistent with the previous study of real-world lower extremity injury study with knee airbag, where an increased risk of tibia/fibula (Risk Ratio: 1.23, 95\% Confidence Interval: 0.52-2.90) and foot fracture (RR: 1.96, 95\% Cl: 0.72-5.32) was noted (Patel et al., 2013). Since current study was limited to controlled laboratory test condition with one specific inflatable knee bolster design, direct comparison between the results from this study and real-world injury risk statistics could not be performed.

All tests were performed with the crash dummy positioned in standard driving posture. Previous studies have revealed that the majority of the drivers tend to drive in an out-of-position posture, which would superimpose another effect to the lower limb loadings, and potentially further increase the lower limb injury risk (Morris et al., 2004; Bose et al., 2010). At this juncture, dynamic tests with out-of-position drivers were 
not performed and thus cannot be assessed quantitatively. However, given the current findings of elevated tibia loading and altered tibia moment upon IKB deployment, it is fair to assume out-of-position posture could potentially aggravate the lower limb response. Based on the results from this study, the increase of tibia index for IKB tests was predominantly dependent on the increase in adjusted tibia bending moment MY. The tibia index applied a critical value of $35.9 \mathrm{kN}$ for the axial compressive loading, which made the difference in the tibia loading between IKB and bolster marginal after the normalization. However, the critical value for resultant bending moment was $225 \mathrm{Nm}$, which could directly reflect any change of tibia bending moment that IKB imposes. Additionally, the peak bending moment occurred during the early stage for tests with IKB, when drivers were essentially in an out-of-position posture with close proximity to the panel surface of the deploying IKB. Given the increase of tibia index was directly induced from the IKB deployment, out-of-position tests should be further investigated as IKB deployment could potentially affect the lower extremity posture and increase the injury risk of tibia/fibula and foot/ankle region in a detrimental manner.

This study supported the design concept of a knee airbag that knee airbag could provide protection to the overall occupant by reducing the pelvis forward excursion and chest deflection. From the review of the test results, compared to the standard bolster tests, the IKB reduced the average chest Gs from $89 \%$ to $60 \%$ with the full-powered driver frontal airbag inflator and from $98 \%$ to $66 \%$ with the depowered inflator for FMVSS208 tests. Similar injury reductions were observed for chest deflection. Average chest deflection was reduced from $92 \%$ to $70 \%$ (full powered inflator) and from $97 \%$ to $82 \%$ (depowered inflator). For the belted NCAP tests, the IKB had protective effect on occupant performance for both the full power and depowered driver inflator tests, but the improvement was marginal. Tests with the depowered inflator resulted in an overall increase in injury values, especially for HIC and neck injury values. Specifically, with the depowered driver frontal airbag, average HIC increased from $42 \%$ to $57 \%$ for the IKB tests, compared to standard bolster tests where the increase of average HIC ranged from $50 \%$ to $60 \%$. Additionally, results from this study indicated the overall tradeoff for whole-body injury risk, as IKB increased the injury risk for the tibia/fibula and foot/ankle regions. This finding reveals the necessity for improved knee airbag design to better protect the lower extremity region.

There are several limitations in current study. Firstly, all the tests were performed with the Hybrid III 50th percentile dummy, which created artifactual bending moments due to its bended shape in the instrumentation. Geometric adjustment applied in the study could account for this variation from human lower limb, but the stiff structure inevitably over-estimated the tibia loadings compared to the response of human legs. Advanced THOR dummy could potentially provide a more biofidelic manner, but this yields to future tests to be performed. Tibia index was used as the injury criterion for the evaluation of lower limb 
injury risk, whereas the results were dominated by the moment component from this injury predictor. Moreover, the tibia index was formulated based on the mid-diaphysis region of the tibia, but real-world data indicated most leg fractures of the occupants occur at distal-third region for drivers in frontal crashes (Ivarsson et al., 2008). Regardless of these limitations, the results from the tibia index were deemed appropriate to reveal the increase injury risk of tibia and fibula region from the knee airbag deployment.

The repeatability of the tests could have been improved. One of the assumptions was the depowering of driver frontal airbag would cause negligible effect on the response of lower extremity, which has been proved from the previous analysis. As observed from the tests, the lower extremity response was highly sensitive to multiple factors, including the IKB firing time, the initial posture of the dummy, as well as the input sled pulse acceleration. Delay of the IKB firing in test 797 resulted in a much higher loading of the tibia, which detrimentally affected the lower extremity response under the knee airbag deployment. Variation of initial dummy positioning also led to the resulting difference in tibia bending moment and loadings, and asymmetric loading at both legs in test 801 . Additionally, the dummy response was dependent on the input impulse signal, which in current study, exhibited a bimodal shape instead of a trapezoid curve that is more widely used in dynamic sled tests. Moreover, this study utilized a single inflatable knee bolster design. Significant changes have been made in improving the inflator design and knee airbag cushion deployment pattern, which could possibly improve the lower limb response accordingly. Until more tests are performed with different types of knee airbags, current findings on knee airbag effects could not be universalized.

Since all the tests were performed with the Hybrid-III $50^{\text {th }}$ ATD, the effect of muscle bracing and braking could not be evaluated, as current test surrogates lack the bio-fidelity of active muscle response of the occupant. Active muscle effects may have the potential to alter the biomechanics of the lower extremities during their interaction with the knee airbag by imparting large internal forces during muscle contraction, or changing the initial position or resulting kinematics of the lower extremities to a configuration different than intended for the design of the knee airbag. High incidence of pre-crash bracing has been indicated in more than two-thirds of occupants in frontal crashes who sustained lower extremity injuries (Petit et al., 1998; Ore et al., 1992). Another recent study also found that muscle activation had a statistically significant effect $(p<0.05)$ on the increasing of the axial force and bending moment of the lower extremity with the deployment of the knee airbag (Nie et al., 2015). Active muscle response could contribute a substantial portion of the loading to the lower extremities, and influence the tibia flexion angle phasing during the deployment of the knee airbag. 
Another factor that remains to be investigated in future was the sensitivity of lower extremity response subject to the vehicle compartment geometry and knee airbag design. Current dynamic tests utilized a vehicle buck typical of a mid-size sedan profile with a production inflatable knee bolster, and were deemed representative of the real-world situations. However, the variation in geometry between vehicle types could change the lower limb driving posture, and lead to a difference on the corresponding lower limb injury risk. Previous study has found that relative to passenger cars, vans exhibited a protective effect against sustaining lower limb injuries, while no association was shown for light trucks or SUVs in real-world crashes (Ye et al., 2015). With the presence of knee airbag, the lower limb injury risk as a function of vehicle type could also change. Additionally, the change in knee airbag geometry, inflator pressure, mounting position and deployment pattern could all potentially result in a disparity in the lower extremity injury risk.

\subsection{Conclusion}

This study analyzed the lower extremity response subjected to a deploying inflatable knee bolster under dynamic condition. A total number of seven unbelted FMVSS208 tests and eight belted NCAP tests were performed with a $50^{\text {th }}$ percentile Hybrid-III dummy, with IKB assembled and deployed in half of tests for paired cross-comparison. For unbelted FMVSS208 tests, tibia index ranged from 0.50 to 1.04 for tests with standard bolster, and 0.60 to 1.21 for tests with inflatable knee bolster. The average tibia index increased by $34.6 \%$ and 33.3\% for tests with IKB on left upper tibia and left lower tibia respectively, compared to tests with standard knee bolster. Additionally, right upper tibia index increased by $20.3 \%$ with the presence of IKB, but right lower tibia index decreased by $26.5 \%$. For belted NCAP tests, tibia index ranged from 0.59 to 1.09 for tests with conventional knee bolsters, and from 0.56 to 1.23 for tests with IKB. The average tibia index increased in left upper, left lower and right upper tibia with the presence of an IKB. The peak tibia bending moment MY directly induced by IKB deployment was the predominant contributing factor for the increase of tibia index.

The results rejected the null hypothesis, and supported the alternative hypothesis that between standard knee bolsters and knee airbags, differences exist in associated injury risk of the tibia/fibula and foot/ankle region for drivers in frontal motor vehicle crashes. The tested inflatable knee bolster changed the driving posture during the deployment stage, and tend to place the lower extremity in a more vulnerable outof-position posture subject to tibia/fibula and foot/ankle injuries. This study reveals the increased injury risk of lower extremity imposed by an IKB during the deployment time in frontal crashes. Future design of the knee airbags should be focused on the improved protection of lower extremity, in order to provide driver safety without detrimentally affecting the tibia/fibula and foot/ankle region. 


\section{CHAPTER 3: Analysis of Knee Airbag Tests for Out-Of-Position Drivers}

\subsection{Motivation}

\section{Out-of-Position Issues}

Out-of-position (OOP) denotes a situation where the vehicle occupants sit at a driving posture different from the standardized posture. OOP could be caused by both involuntary reaction to vehicle motion and conscious muscle activation in attempt to control of motion. OOP cases could be found where occupants are located unusually close to the airbag module at the time of inflation. Previous study indicated that occupants do not generally maintain the nominal posture orientation as Anthropometric Test Device (ATD) in standardized crash tests, and tend to change the driving posture in traffic situations (e.g., under the stressful conditions of pre-impact braking or bracing). Consequently, the response of restrained system could vary and the efficiency is reduced (Bingley et al., 2005). Another previous study on 49 volunteers indicated that occupant posture depends on a wide variety of factors including general state of mind, vehicle conditions, long duration tasks and personal preferences (Morris et al., 2004). Besides the human-induced factors, out-ofposition posture could also result from delayed deployment of the airbag, improper seatbelt use, and initial seating placement with closer proximity to the instrument panel (Malczyk et al., 1995). Significant differences were observed between relaxed driving posture and standardized posture, and out-of-position posture was noted more common for occupants of smaller anthropometric sizes, as small occupant's knees are placed closer to the knee bolster module (Schneider et al., 1983). The injury risk of lower extremity is generally higher, and the less efficiency of the knee airbag system is capable to provide.

Disconnect exists between driving posture reflected in crash tests and real-world driving scenarios. Studies indicated only $17 \%$ of drivers were close to the nominal driving posture (Morris et al., 2005; HaultDubrulle et al., 2011). Moreover, over $60 \%$ of all drivers tend to brake or brace during an emergency, which lead to the change in driving posture (Ore et al., 1992). Additionally, approximately $90 \%$ of U.S. adult drivers were associated with incorrect seatbelt use, with the lap belt being placed further forward and higher relative to the pelvis (Reed et al., 2013).

The wide variation in driving posture demonstrated the necessity to perform out-of-positon tests as an approach to fully examine the effectiveness of countermeasures. When the scope was narrowed down to lower extremity region, studies found that human subjects placed their knees approximately twice as far apart in the horizontal plane than the procedure established by 208 for positioning ATD's. Therefore, the volunteer's knees are positioned over different bolster locations than the ATD's 208 position (Atkinson et al., 2009). 
Another study indicated similar results that the knee to knee bolster distance vary between drivers in different posture, which is likely to affect the knee excursion across the knee location variation (Reed et al., 2014).

Combing these findings, the percentage of drivers who maintain the identical nominal driving posture as specified in standardized crash tests was marginal. Several studies with out-of-position tests were performed previously, but little information is readily available regarding the knee airbag effectiveness on the variance in driver lower limb posture. More importantly, previous study of IKB dynamic tests indicated that tibia bending moment MY was the predominant factor to increase the tibia index and associated tibia/fibula injury risk, while the peal tibia bending moment mostly occur during the IKB deployment stage when drivers were actually in an out-of-position posture. The following study was derived based on this finding, and intentionally place the driver in a more vulnerable out of-position with closer proximity to the knee airbag, as an approach to represent the worst-case scenario and to investigate the corresponding lower extremity risks. The following manuscript presented the study of investigating the lower extremity response for out-of-position drivers under knee airbag static deployment (Ye et al., 2014).

\subsection{Paper Manuscript}

\section{Lower Extremity Response for Out-of-Position Drivers under Knee Airbag Deployment}

Abstract The $5^{\text {th }}$ percentile female was chosen for an investigation of knee loading resulting from interaction with a deploying knee airbag. In this study, a total of 11 static knee airbag deployment tests were performed with a $5^{\text {th }}$ percentile female Hybrid-III dummy outfitted with either the original Hybrid-III lower extremities or the $5^{\text {th }}$ percentile THOR-FLx. Baseline tests were performed with FMVSS 208 seating specifications, and a design of experiment for out-of-position conditions was developed with multiple factors including knee-to-instrument panel distance, knee-to- knee distance, and foot placement. The upper tibia index values ranged from 0.95 to 1.31 , and 0.78 to 1.21 for baseline tests of Hybrid-III LX and THOR-FLX, respectively. Lower tibia index values varied from 0.30 to 0.46 (Hybrid-III) and from 0.51 to 0.79 (THOR-FLx). For baseline tests, highest injury risk of AIS 2+ leg shaft fractures occurred in upper right tibia of Hybrid-III LX (31.15\%) and in upper left tibia of THOR-FLx (51.17\%). Translating the dummy to the full-forward position with the tibia contacting the knee bolster resulted in an average $\mathrm{TI}$ increase of $10 \%$ and greater abduction of both legs during knee airbag deployment. With the right foot moved inboard from accelerator to brake pedal, the average $\mathrm{TI}$ increased $120 \%$ relative to baseline. Overall, the highest average $\mathrm{TI}$ was recorded with the left foot moved inboard creating an adducted initial position. The results also predicted higher injury risk of tibia shaft fractures than foot and ankle fractures. The elevated dummy lower extremity response recorded in this study 
suggests considerations to be made for out-of-position small female occupant response during knee airbag deployment.

Keywords Injury risk, knee airbag, lower extremity, out-of-position

\section{INTRODUCTION}

Currently, 45\% of AIS 2+ injuries for occupants involved in frontal crashes occur in the lower extremities (Ye et al., 2015). Although improvements in occupant safety have resulted in a reduction of head and chest injuries over the past 15 years, the rate of lower limb injuries over this time has remained virtually unchanged. This finding contracts with frontal crash tests data that has shown significantly decreased vehicle measures (e.g., toe pan intrusion), and responses measured in the dummy have steadily decreased during the same period.

Given the prevalence of lower limb injuries and the fact that current vehicle modifications (e.g., structural modifications to reduce intrusion) have not reduced incidence rates, consideration must be given to other available countermeasures that could mitigate lower limb injuries. In addition to controlling occupant kinematics through earlier engagement of the pelvis, knee airbag (KAB) has reportedly been designed and developed to prevent lower limb injuries. Jenkins et al. discussed the structural improvement of conventional knee bolster material from steel brackets to engineering plastics with the implementation of an inflatable knee bolster (Jenkins et al., 2002). This change may also provide extra space for lower limb placement and ultimately for more energy absorption. Knee airbags deployment along the lower panel fascia can restrain the knees during early phases of the crash and can also help reduce the loading to upper tibia (Ohachi et al., 2012).

Two recent publications have analyzed data from the Crash Injury Research and Engineering Network (CIREN) and National Automotive Sampling System Crashworthiness Data System (NASS-CDS) to evaluate realworld lower extremity injury risk in frontal crashes. Weaver et al. compared 9 CIREN cases with knee airbag to 183 no-knee airbag cases of the same vehicle model, crash type, and severity using a similarity scoring algorithm (Weaver et al., 2013). Results indicated a statistically significant reduction in femur fractures, but an increased incidence rate of proximal tibia/fibula and foot/ankle fractures (also statistically significant), for occupants in crashes with deployed knee airbags. While the study by Patel et al. combined NASS-CDS and CIREN data to maximize case availability for a matched cohort study, there were still an insufficient number of cases to find statistical significance of the lower extremity injury risks, although a decreased risk of hip and thigh fracture, and an increased risk of tibia/fibula and foot fracture were identified (Patel et al., 2013). 
Beyond these two studies, relatively little has been published regarding the performance of knee airbags in real-world crashes, despite their increasing market penetration. Part of the difficulty results from the fact that there are a multitude of different airbag configurations (bottom-deployed or rear-deployed, high-mount or low-mount, KAB size, inflator output, etc.) that confound the assessment of KAB performance in retrospective field studies. Given the lack of understanding of real-world performance, questions arise regarding the knee airbag effectiveness in different frontal crash scenarios, the limitations of airbag coverage and overloading, the changes in injury patterns relative to knee bolsters, and the potential for lower limb injuries resulting from deployment of the knee airbag itself. This study aimed to investigate the biomechanical response of the lower extremities during knee airbag deployment in various out-of-position driving scenarios. Specifically, this study used dummy tests to assess the potential for out-of-position lower extremity injuries upon knee airbag deployment and to observe how knee airbag deployments could alter the occupant's positioning during a crash.

\section{METHODS}

Among standard adult dummies, the 5th percentile female occupants were chosen to represent the most vulnerable group for sustaining lower limb injuries, given their diminished lower injury tolerance and a closer seating proximity to the instrument panel/ knee bolster (Mertz, 1993; Reed et al., 2014). Therefore, the $5^{\text {th }}$ percentile female was the target occupant for an investigation of knee loading resulting from interaction with a deploying knee airbag.

The current study consisted of 11 knee airbag static deployment tests, with a $5^{\text {th }}$ percentile female Hybrid-III dummy seated in a simplified vehicle buck. The simplified test buck was designed to match the dimensions typical of a production small compact sedan profile. The buck structure consisted of occupant seat, instrument panel, knee bolster, toe pan structures, and pedals. The non-production knee airbag used in this test series was a rear-deploy type, mounted on the reinforced instrument panel of the simplified buck. The knee airbag assembly included the housing, cover, and inflator module (ARC hybrid gas inflator, 194kPa maximum tank pressure at $24.65 \mathrm{~ms}, 28.3 \mathrm{~L}$ tank volume and 0.9 mole). Vent holes in the airbags were initially blocked by stitching as unvented knee airbags represent a larger number of modules in the field. Details regarding the knee airbag are provided in Appendix 2.

All tests were performed in a static condition. The dummy was positioned in various out-of-position configurations representative of potential worst-case scenarios in frontal crashes, and in-position baseline tests with occupant positions comparable to specifications in the Federal Motor Vehicle Safety Standard (FMVSS) 208 (Figure 43). Positioning of the seat also matched with FMVSS 208 test for a $5^{\text {th }}$ percentile female dummy (i.e., forward most position in seat track, mid-height) (Lewandowski et al., 2007). The seat was then 
fixed and dummy was translated on the seat for various out-of-position postures. The seat was a simplified wooden rigid plate, with supporting structures made of steel and geometry (height, inclination angle) equivalent to the standardized test, but with less energy absorption and no anti-submarining structure than a production vehicle seat.
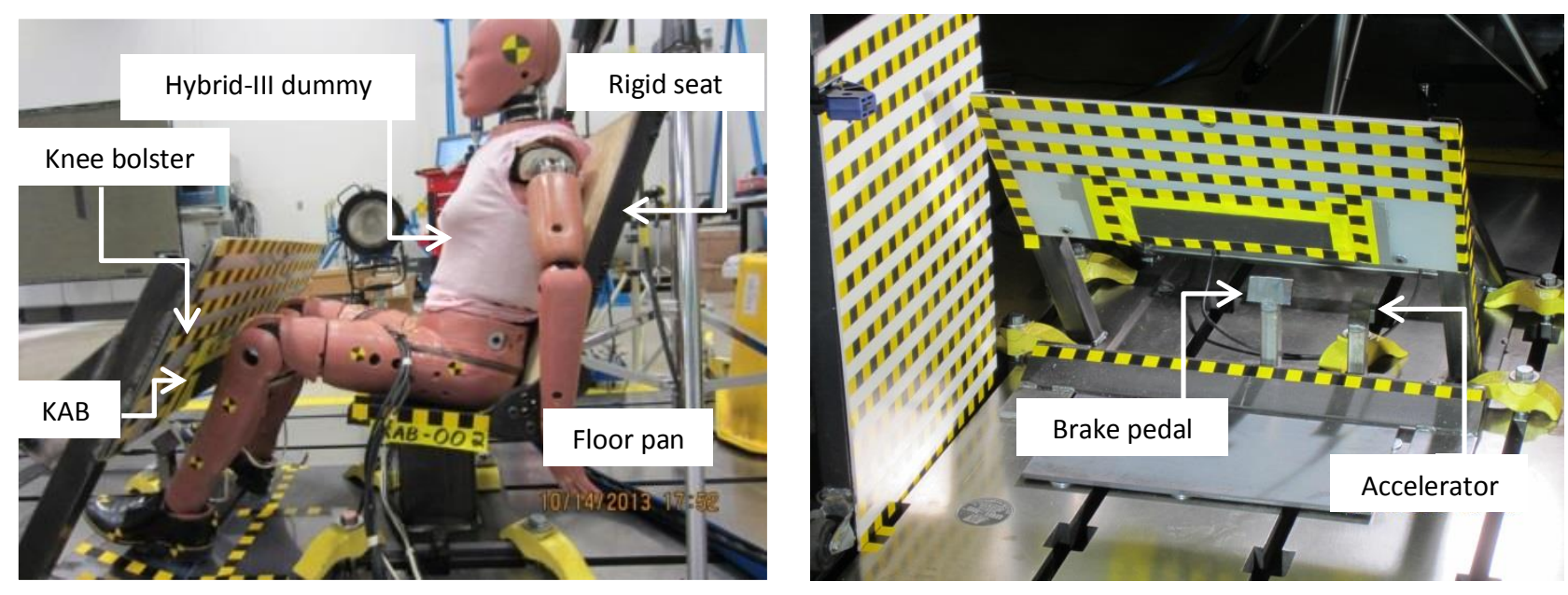

Figure 43. Schematic of test-setup with positioned dummy (Left); Overview of driver compartment (Right)

The Hybrid-III $5^{\text {th }}$ female test matrix included two dummy lower extremities: the 5 th percentile female Hybrid-III with the Hybrid-III Denton lower leg and the advanced THOR-FLx. THOR-FLx was retrofitted to the distal femur of the 5th percentile female Hybrid-III dummy as a more biofidelic testing device (Rudd et al., 2003). Components from the THOR-FLx are mostly scaled representations of the original $50^{\text {th }}$ percentile male THOR-Lx counterparts. New design aspects related to the THOR-FLx included modifications to the tibia axial compliance, the elastomeric stops at two principal ankle-joint stops, the Achilles tendon, the anterior tibia shape, and the side knee covers attached to the knee clevis (Shams et al., 2002). Given the identical input test conditions, this parametric study investigated the similarity of response between Hybrid-III Denton leg and THOR-FLx and analyzed any potential differences during KAB interaction.

Regarding dummy positioning, baseline tests generally matched the FMVSS 208 in-position tests for the $5^{\text {th }}$ percentile Hybrid-III dummy. Dummy calibration and polarity testing were performed before positioning, while the posture measurements were taken using a 3D coordinate measurement machine (FARO Technologies, Lake Mary, FL, USA), as well as traditional measurement tools including calipers, tape measures and inclinometers. Markers were placed at multiple locations of both left and right extremities to capture the postures for scanning measurements of pre-test posture. The dummy femurs and tibias were painted and chalked to observe contact with the knee airbag. The dummy upper extremities were placed vertically in line with the torso to avoid interference with lower extremity movement.

Table 5 below showed the test matrix in this study. A total of 11 tests were conducted, with four test configurations for both Hybrid-III and THOR-FLx dummy legs, and repeated baseline tests. An additional test 
(THOR-ADD1) was repeated from an earlier test (THOR-ADD) as it was noticed in post-test analysis that the right hind-foot in test THOR-ADD was elevated above the floor pan rather than resting on it. A detailed summary of dummy positioning is listed in Appendix 1.

Table 5. Knee airbag test matrix

\begin{tabular}{|c|c|c|c|c|c|c|c|}
\hline Num & Test & Dummy leg & $\begin{array}{c}\text { Knee to } \\
\text { instrument } \\
\text { panel }(\mathrm{mm})\end{array}$ & $\begin{array}{l}\text { Knee to knee } \\
\text { dist. }(\mathrm{mm})\end{array}$ & $\begin{array}{l}\text { Right foot } \\
\text { placement }\end{array}$ & $\begin{array}{l}\text { Left foot } \\
\text { placement }\end{array}$ & Comments \\
\hline 01 & H3-BAS1 & Hybrid-III & $\begin{array}{l}\text { Right: } 85 \\
\text { Left: } 82\end{array}$ & 252 & accelerator & footrest & Baseline\#1 \\
\hline 02 & H3-BAS2 & Hybrid-III & $\begin{array}{l}\text { Right: } 85 \\
\text { Left: } 82\end{array}$ & 252 & accelerator & footrest & Baseline\#2 \\
\hline 03 & H3-FWD & Hybrid-III & $\begin{array}{l}\text { Tibia contacts } \\
\text { knee bolster }\end{array}$ & 252 & accelerator & footrest & $\begin{array}{c}\text { Dummy translated } \\
\text { full-forward }\end{array}$ \\
\hline 04 & H3-ADD1 & Hybrid-III & $\begin{array}{l}\text { Tibia contacts } \\
\text { knee bolster }\end{array}$ & 180 & $\begin{array}{l}\text { brake } \\
\text { pedal }\end{array}$ & footrest & $\begin{array}{l}\text { Dummy at full- } \\
\text { forward }\end{array}$ \\
\hline 05 & H3-ADD2 & Hybrid-III & $\begin{array}{l}\text { Tibia contacts } \\
\text { knee bolster }\end{array}$ & 158 & $\begin{array}{l}\text { brake } \\
\text { pedal }\end{array}$ & footrest & $\begin{array}{l}\text { Adducted, left foot } \\
\text { inboard }\end{array}$ \\
\hline 06 & THOR-BAS1 & THOR-FLX & $\begin{array}{l}\text { Right: } 85 \\
\text { Left: } 82\end{array}$ & 252 & accelerator & footrest & Baseline\#3 \\
\hline 07 & THOR-BAS2 & THOR-FLx & $\begin{array}{l}\text { Right: } 85 \\
\text { Left: } 82\end{array}$ & 252 & accelerator & footrest & Baseline\#4 \\
\hline 08 & THOR-FWD & THOR-FLx & $\begin{array}{l}\text { Tibia contacts } \\
\text { knee bolster }\end{array}$ & 252 & accelerator & footrest & $\begin{array}{l}\text { Dummy translated } \\
\text { full-forward }\end{array}$ \\
\hline 09 & THOR-ADD & THOR-FLx & $\begin{array}{l}\text { Tibia contacts } \\
\text { knee bolster }\end{array}$ & 180 & $\begin{array}{l}\text { brake } \\
\text { pedal }\end{array}$ & footrest & $\begin{array}{c}\text { Dummy at full- } \\
\text { forward, right heel } \\
\text { elevated }\end{array}$ \\
\hline 10 & THOR-ADD2 & THOR-FLX & $\begin{array}{l}\text { Tibia contacts } \\
\text { knee bolster }\end{array}$ & 158 & $\begin{array}{l}\text { brake } \\
\text { pedal }\end{array}$ & footrest & $\begin{array}{l}\text { Adducted, left foot } \\
\text { inboard }\end{array}$ \\
\hline 11 & THOR-ADD1 & THOR-FLx & $\begin{array}{l}\text { Tibia contacts } \\
\text { knee bolster }\end{array}$ & 180 & $\begin{array}{l}\text { brake } \\
\text { pedal }\end{array}$ & footrest & $\begin{array}{l}\text { Dummy at full- } \\
\text { forward }\end{array}$ \\
\hline
\end{tabular}

SAE Channel Frequency Class $600 \mathrm{~Hz}$ filter was applied to the force and moment data and SAE Channel Frequency Class $180 \mathrm{~Hz}$ filter was applied to displacements following the SAE J211 standard. Data were all reported in accordance with the SAE coordinate convention. Data recording started $20 \mathrm{~ms}$ prior to the knee airbag firing time and ended $150 \mathrm{~ms}$ after firing. Three high-speed cameras (Memrecam GX-3, NAC Image 
Technology, California, USA) documented the deployment process with a frame rate of 2000 frames per second for kinematics analysis.

For the calculation of tibia index (TI), a geometric adjustment of the tibia sagittal moment was performed for the upper and lower tibia load cell locations of the Denton leg. The adjustment compensated for the nonanatomical geometry of the Hybrid-III dummy lower extremity, which can result in an over-estimation of proximal tibia fracture (Zuby et al., 2001). The adjustment function was shown below, with the geometrical coefficients measured from the $5^{\text {th }}$ percentile dummy (Kuppa et al., 2001).

$$
\begin{aligned}
& M y_{\text {upper.adj }}=M y_{\text {upper.meas }}-\left(F z_{\text {upper }}\right)(0.01589) \\
& M y_{\text {lower.adj }}=M y_{\text {lower.meas }}+\left(F z_{\text {lower }}\right)(0.004665)
\end{aligned}
$$

The units for the compensation force and moments were force in $\mathrm{N}$ and moment in $\mathrm{Nm}$. The resultant moment was calculated after the adjustment of moment for the Hybrid-III leg. Tibia index was calculated for the Hybrid-III leg, and the revised TI was calculated for the THOR-FLx dummy leg as indicated below (Kuppa et al., 2001).

$$
\begin{aligned}
& M_{r}=\sqrt{M_{x}^{2}+M_{y}^{2}} \\
& T I_{\text {Hybrid-III }}=\frac{F_{z}}{F_{c}}+\frac{M_{r}}{M_{c}}=\frac{F_{z}}{(-22900)}+\frac{M_{r}}{115} \\
& T I_{\text {THOR-FLx }}=\frac{F_{z}}{F_{c}}+\frac{M_{r}}{M_{c}}=\frac{F_{z}}{(-8600)}+\frac{M}{146}
\end{aligned}
$$

Where $M_{r}$ is the resultant tibia moment, $F_{z}$ is the tibia compression force, $F_{c}$ and $M_{c}$ are the critical threshold values for force and moment in tibia index. TI was calculated using compression force and resultant moment responses for the upper and lower, left and right tibia load cell locations.

Multiple injury risk functions were used to estimate the injury risk of AIS 2+ lower extremity injuries, including tibia shaft fractures, knee-thigh-hip injuries, ankle fractures, and tibia plateau injuries. Scaling of the injury risk function was applied to account for the geometry and mass differences between the $50^{\text {th }}$ percentile and the $5^{\text {th }}$ percentile dummies (Kuppa et al., 2001).

Injury Assessment Reference Values (IARV) obtained from Insurance Institute for Highway Safety (IIHS) for the $5^{\text {th }}$ percentile dummy were applied to the test data as a guideline for evaluating injury measures (IIHS, 2014). 


\section{RESULTS}

The deployment of the knee airbag was composed of several phases as indicated by the sequence of time-lapsed photos (Figure 44 and Figure 45). After triggering of the knee airbag, the cover of the airbag was breached and the airbag started unfolding. Initial contact of the airbag with the occupant started with the upper tibia region and migrated upwards to the knee region. The airbag unfolded on the medial side of each knee simultaneously and abducted both legs. The closed vent holes ruptured in three tests (H3-ADD1, H3ADD2, and THOR-ADD2); while in the other tests the knee airbags remained intact. The final stage of knee airbag interaction with the occupant occurred with the knee airbag wrapped over both knees of the dummy.

\section{Starting point of trigger $(\mathrm{t}=0 \mathrm{~ms})$}

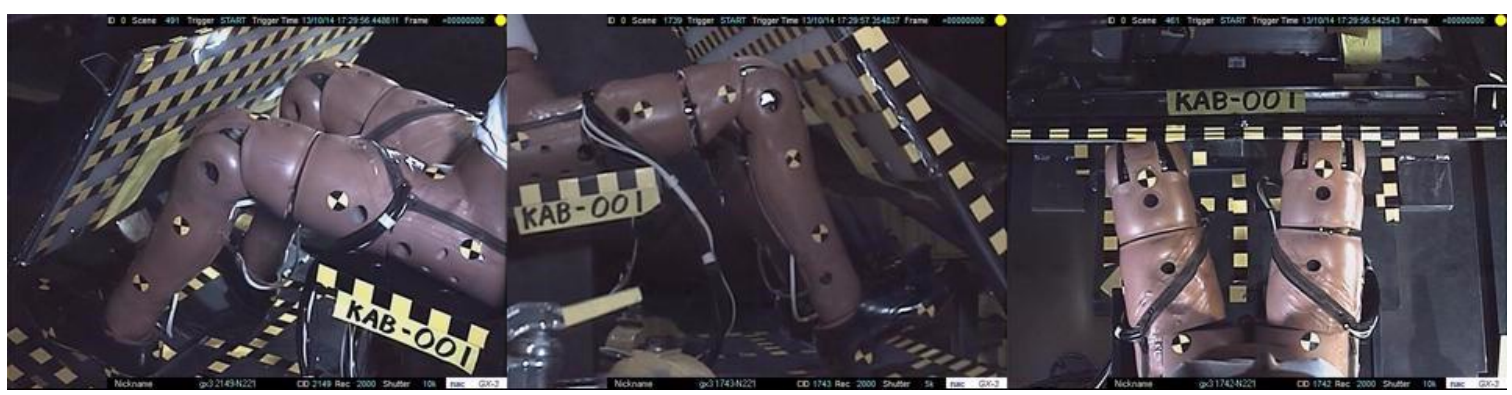

2. Airbag unfolded and contacted upper tibia region ( $t=5 \mathrm{~ms}$ )

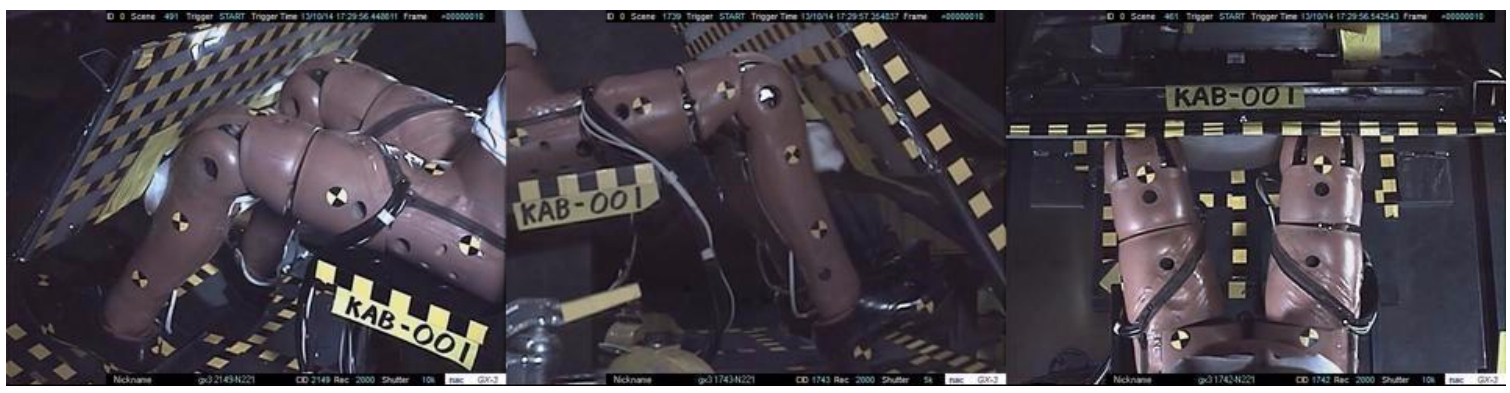

3. Airbag deployed and contact knee region ( $t=10 \mathrm{~ms}$ )

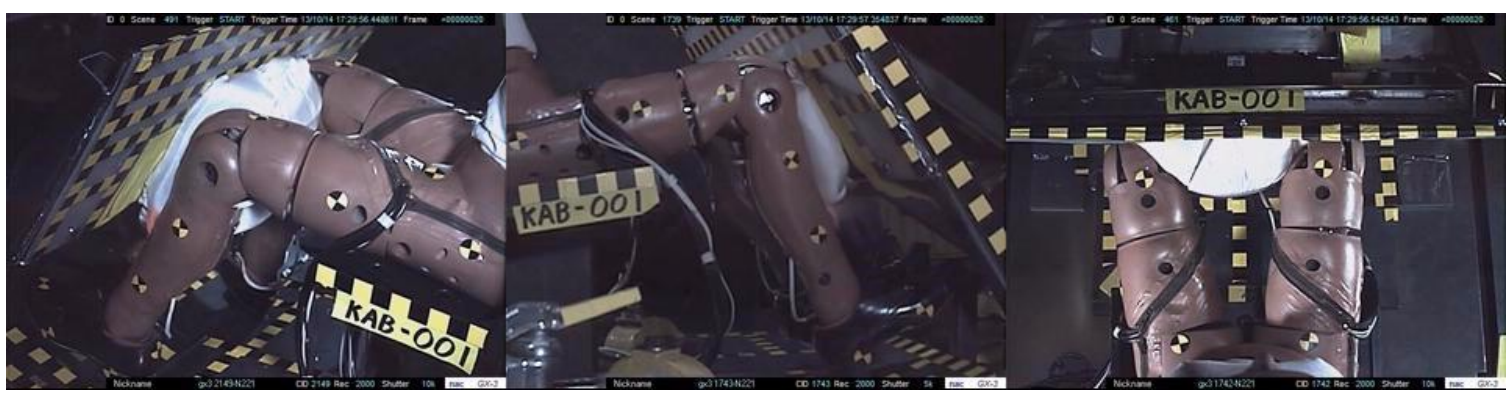




\section{Unfolding continuation ( $\mathrm{t}=15 \mathrm{~ms})$}

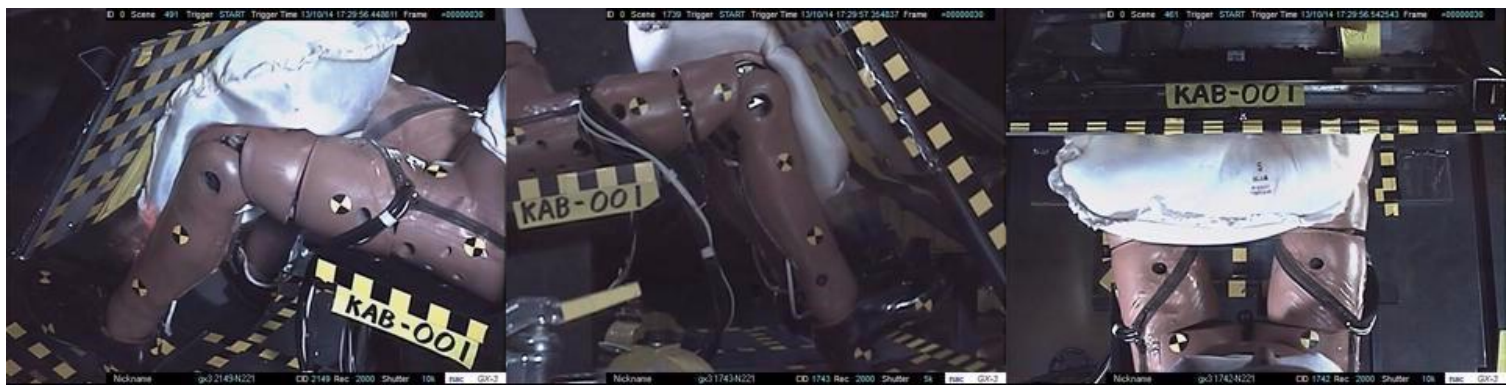

Figure 44. Typical response of the knee airbag during the deployment phase

5. Abduction of both legs due to airbag deployment ( $t=20 \mathrm{~ms})$

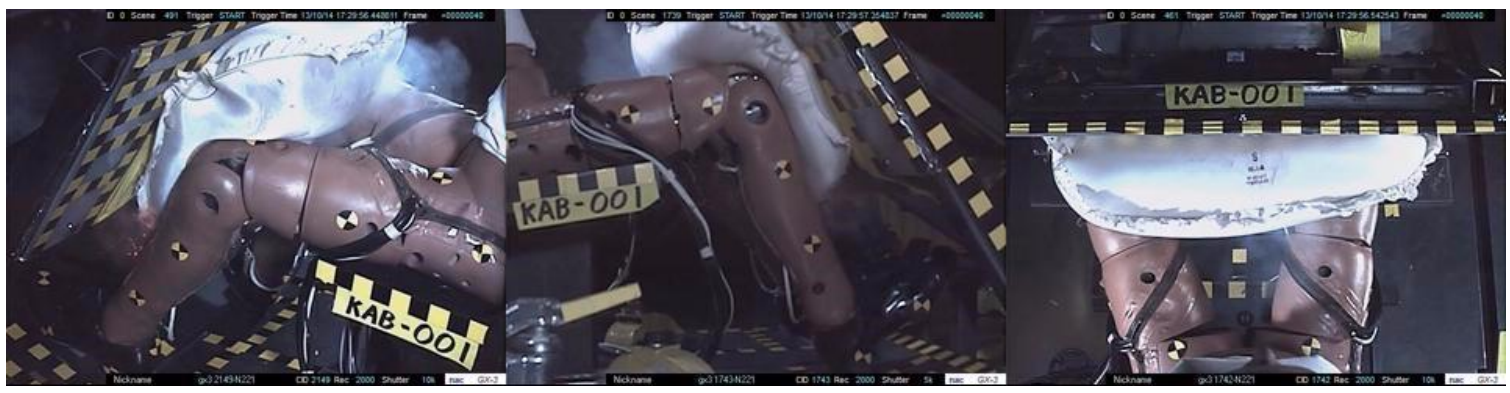

6. Full contact with lower extremity $(\mathrm{t}=25 \mathrm{~ms})$

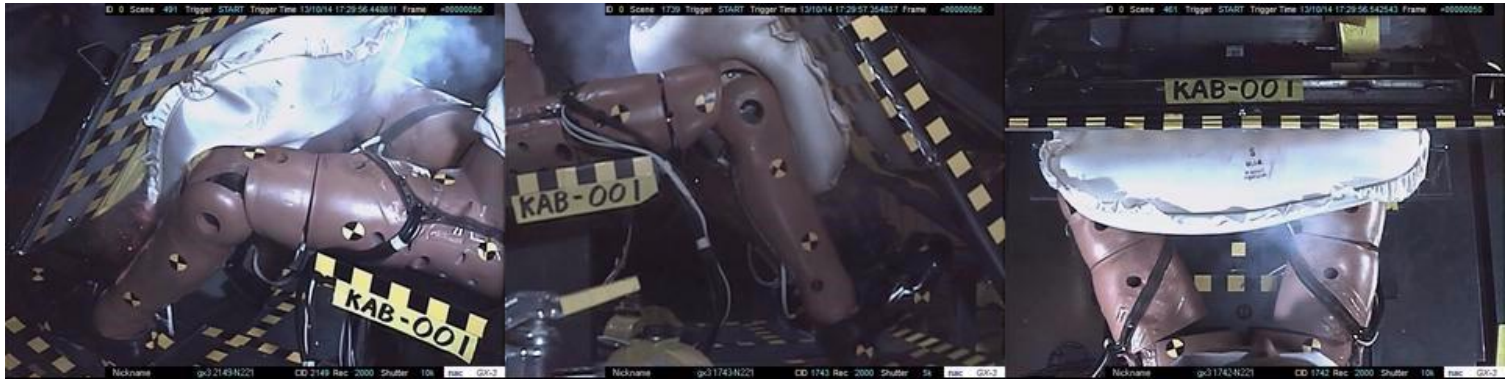

7. Opening of vent holes (for some tests) ( $t=30 \mathrm{~ms}$ )

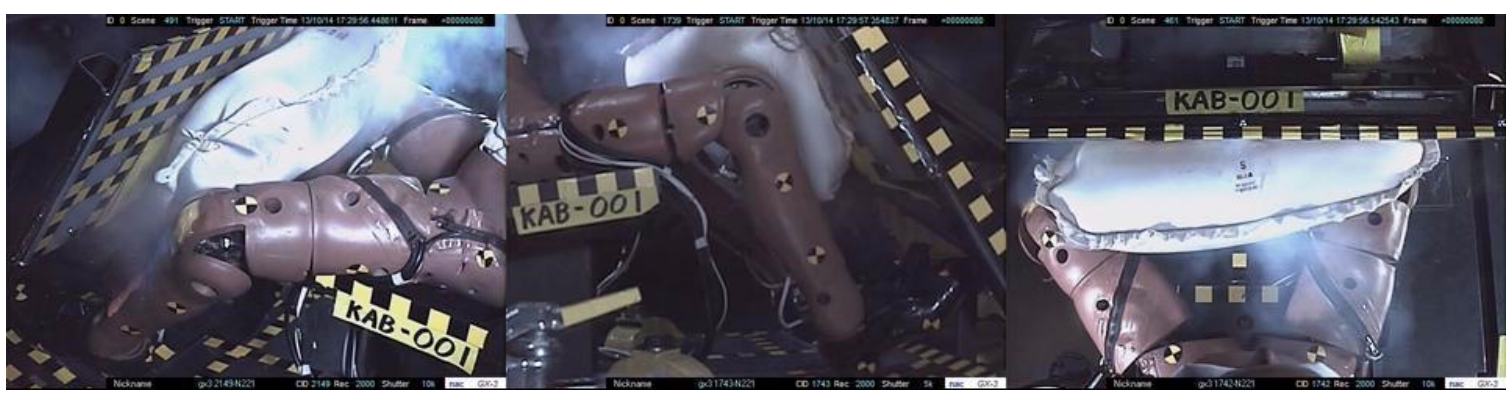


8. Airbag wrapped over the knees at final stage $(t=50 \mathrm{~ms})$

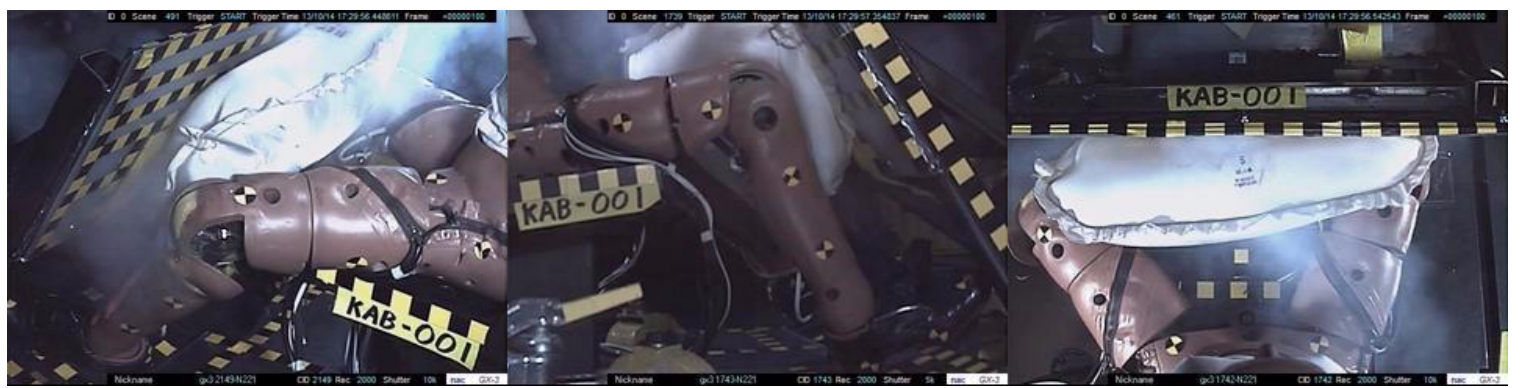

Figure 45. Typical kinematic response of the dummy following knee airbag deployment

The lower left tibia compression force $F_{z}$ from the THOR-FLx was consistently higher than those in the Hybrid-III, with an average difference of more than 75\% in magnitude (Figure 46). Repeated baseline inposition tests with the Hybrid-III leg showed similar responses with peak left lower tibia compressive force at $1382 \mathrm{~N}$ and $1264 \mathrm{~N}$. When translating the dummy full-forward and maintaining the knee-to-knee distance, maximum force decreased slightly to $900 \mathrm{~N}$. With the dummy at the full-forward position and both thighs adducted, the highest compression loading of $2397 \mathrm{~N}$ (H3-ADD2) occurred in the lower left tibia for the Hybrid-III leg, an increase of $45 \%$ over the average of the two baseline tests. A similar trend was observed in THOR-FLx tests, with initial peak force from the two baseline tests reaching $1843 \mathrm{~N}$ and $1581 \mathrm{~N}$. A second peak force of $2606 \mathrm{~N}$ was observed in test THOR-BAS1. Adduction of the knee at full-forward seating position generated a high compression force of $2928 \mathrm{~N}$ for THOR-FLx; while in test THOR-ADD1, peak force elevated to $3307 \mathrm{~N}$. In addition, more oscillation occurred in both lower and upper left tibia from THOR-FLx tests than Hybrid-III. This is due to the structural difference where axial loading was generated by the Achilles tendon assembly in the THOR-FLx, and additional compression force was superimposed at the lower tibia from this loading path. Lower left tibia moments $M_{x}$ and $M_{y}$ from THOR-FLx showed comparable values to the Hybrid-III tests (Figure 47 and Figure 48).
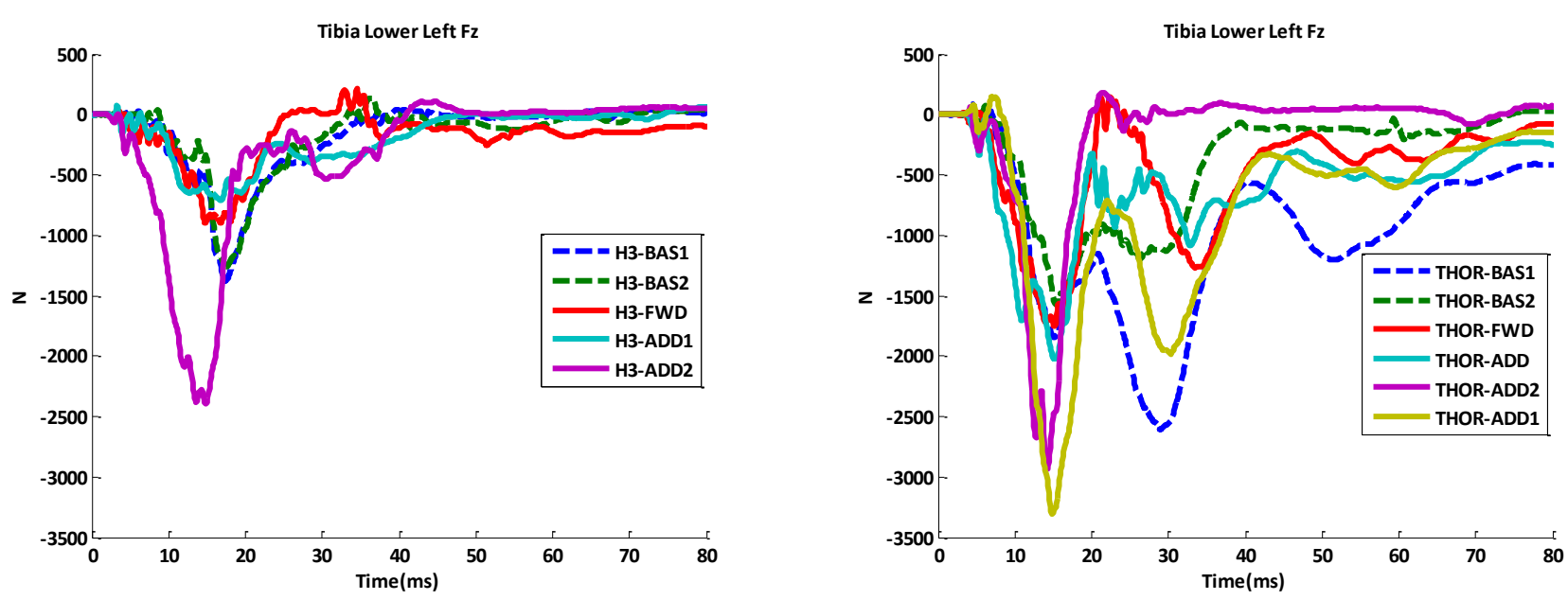

Figure 46. Tibia lower left force Fz in Hybrid-III (left) and THOR-FLx (right) 

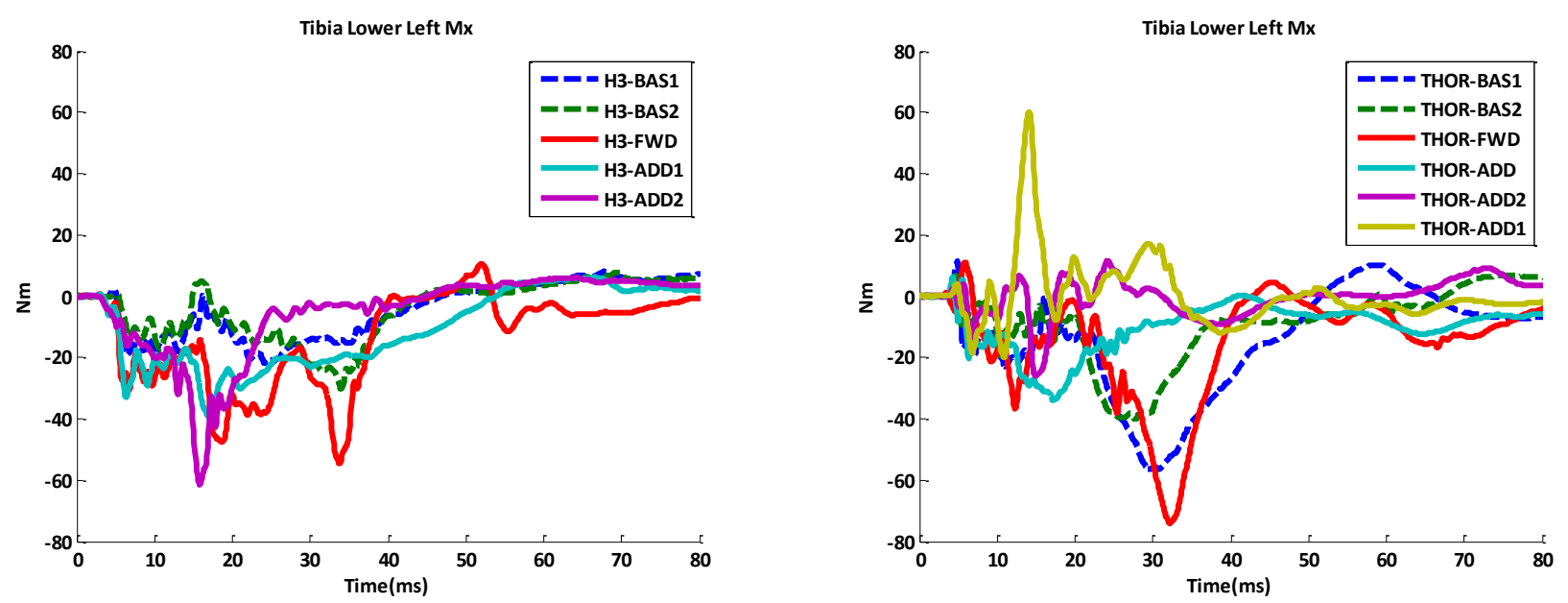

Figure 47. Tibia lower left moment Mx in Hybrid-III (left) and THOR-FLx (right)
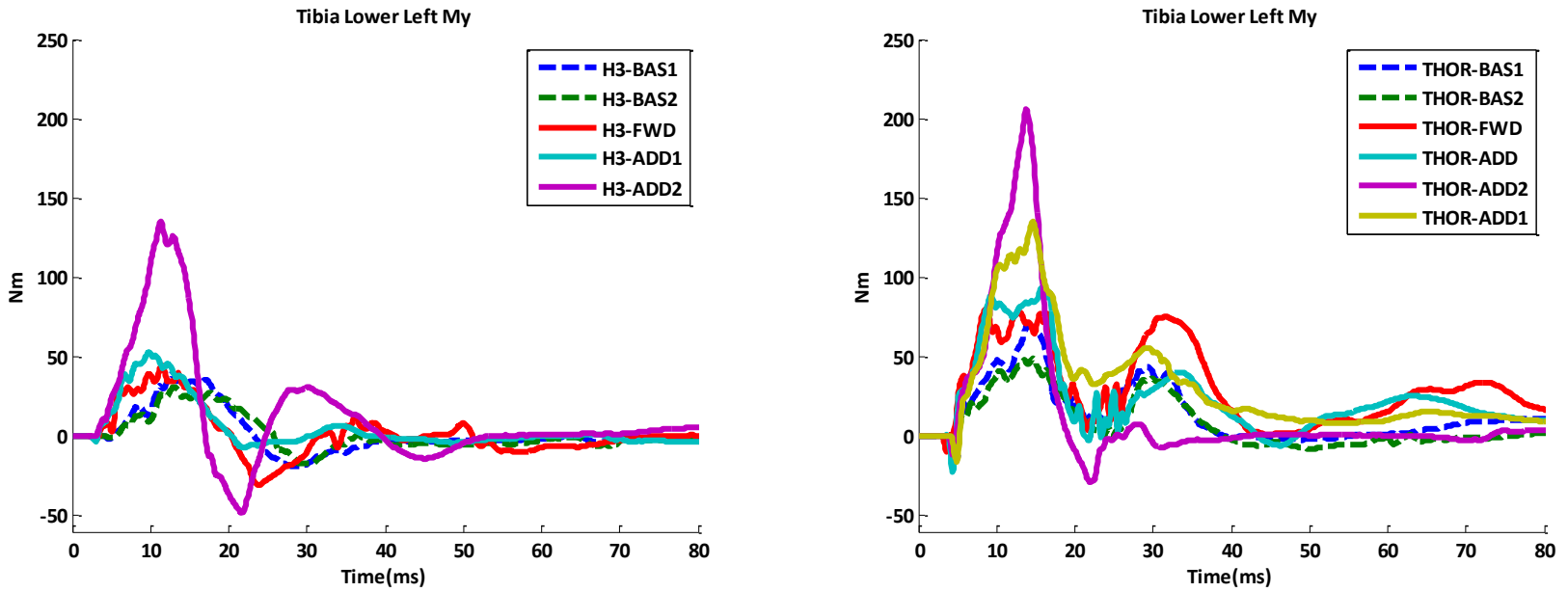

Figure 48. Tibia lower left moment My in Hybrid-III (left) and THOR-FLx (right)

Upper tibia index ranged from 0.95 to 1.31 , and 0.78 to 1.21 for baseline tests of Hybrid-III LX and THORFLx, respectively. Lower tibia index varied from 0.3 to 0.46 (Hybrid-III) and from 0.51 to 0.79 (THOR-FLx) (Figure 49). 


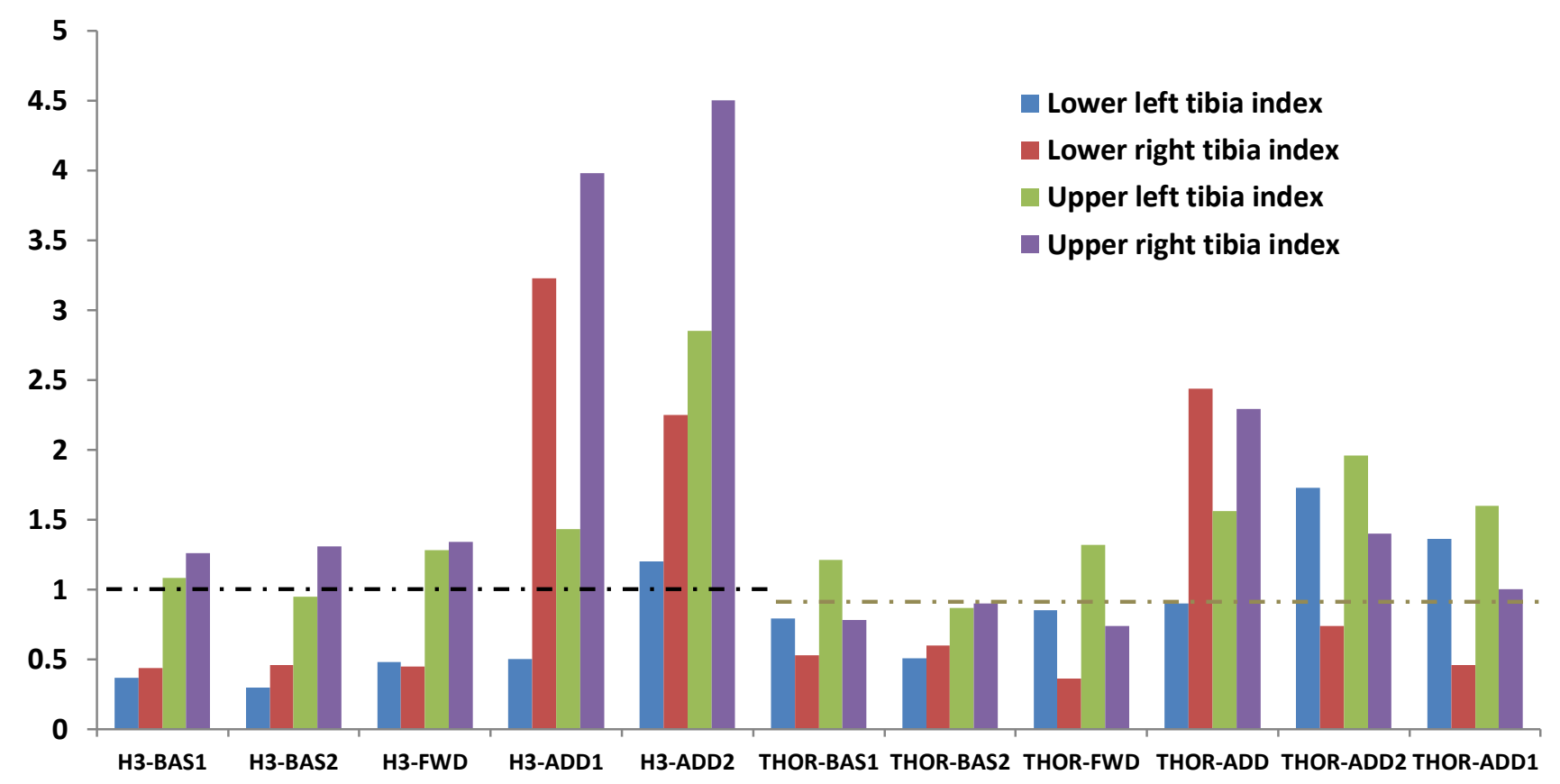

Figure 49. Summary of tibia index for all tests

As depicted in Figure 49 , the average tibia index increased by $15 \%$ for the Hybrid-III and $6 \%$ for the THORFLx in the full-forward position compared to baseline. With the right foot moved inboard from the accelerator to the brake pedal, the average $\mathrm{TI}$ increased 196\% (Hybrid-III) and 43\% (THOR-FLX) relative to baseline tests. Finally, the highest average TI, 250\% (Hybrid-III) and 88\% (THOR-FLx) greater than baseline, was recorded with the left foot moved inboard creating an adducted initial position of the lower limbs. In general, the upper tibia sustained higher bending moments resulting in higher tibia index values, and the right TI was generally higher than the left. As noted in Figure 49, a threshold of 1.0 (black dotted line) was set as the tibia index for HybridIII, and a revised critical value of 0.91 was used(grey dotted line) as the proposed THOR-FLx injury limit [7]. For most out-of-position tests, right TI exceeded the threshold, with the maximum TI of 4.5 occurring in the upper right tibia from test H3-ADD2, mostly resulted from extreme high tibia upper right moment $M_{y}(543 \mathrm{Nm})$.

The results from injury risk functions predicted higher injury risk of tibia shaft fractures than foot and ankle fractures (Figure 50-52). In addition, for the risk of AIS2+ knee-thigh-hip injuries based on left and right axial femur forces, predicted injury risk ranged from $0.35 \%$ to $0.52 \%$ across all the tests, but the difference between tests was not distinctive so the plot was not shown. The increased risk of tibia shaft resulted from the high value of moments upon knee airbag deployment, while relatively less loading was applied to the foot/ankle complex. For test THOR-ADD, due to the elevation of right hind-foot, extremely high compression force was applied to the right leg as the heel was driven downward and landed on floor pan, with lower right tibia axial compression force of $8404 \mathrm{~N}$ and upper right tibia force of $4723 \mathrm{~N}$. This driving posture also resulted in large dorsiflexion and compression of the right foot, while the foot was initially placed on the brake pedal. With 
respect to injury risk of lower extremity, data recorded from Hybrid-III Denton leg was comparable with the retrofitted THOR-FLx. Rudd et al. also found that the lower limb responses between the $5^{\text {th }}$ percentile HybridIII and THOR-FLx leg were less distinct than the differences for the 50th percentile dummy legs, while only the ankle y-axis moment showed clear differences under dynamic sled test conditions [7].

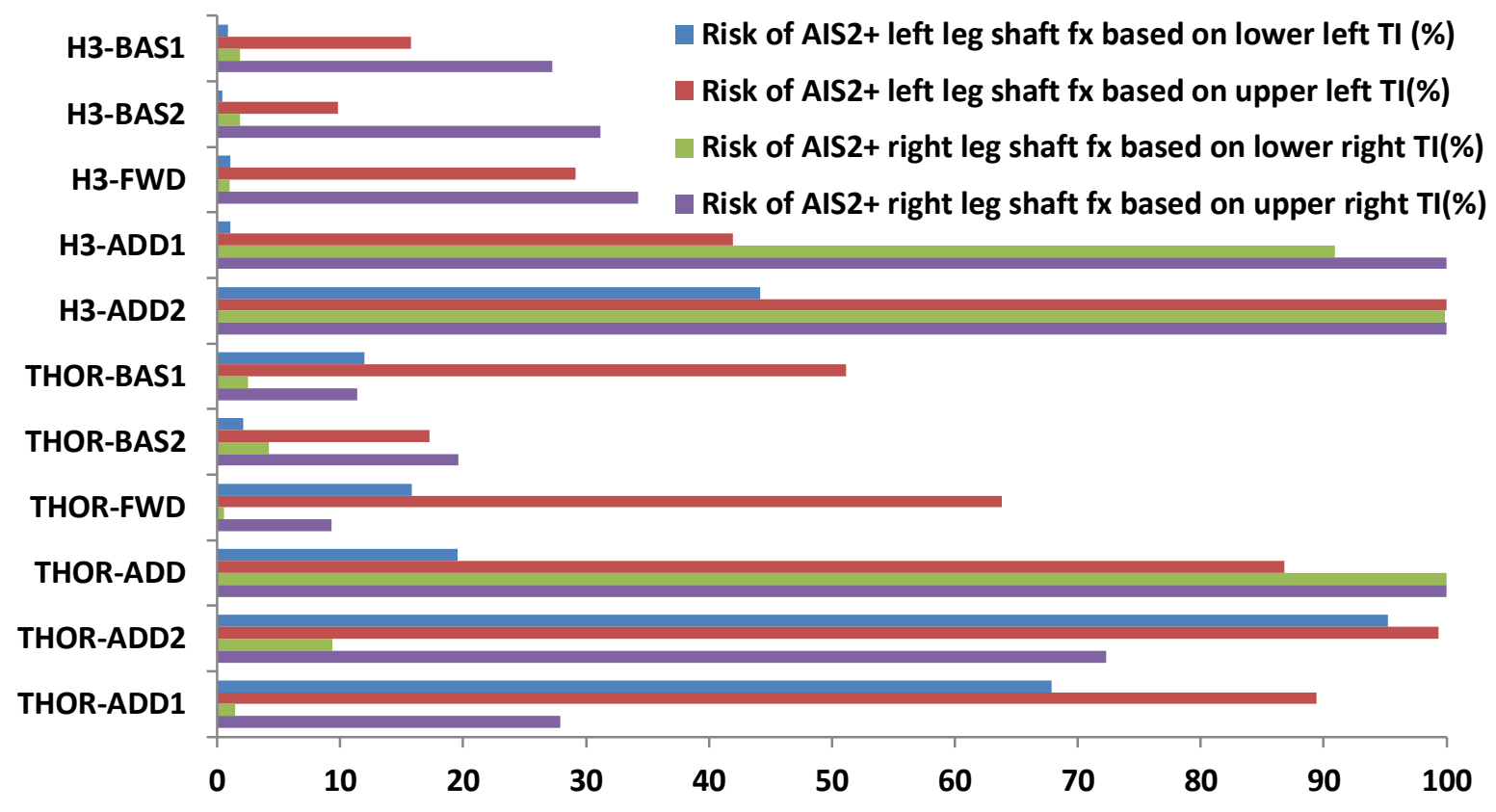

Figure 50. Risk of AIS2+ leg shaft fractures based on tibia index (\%)

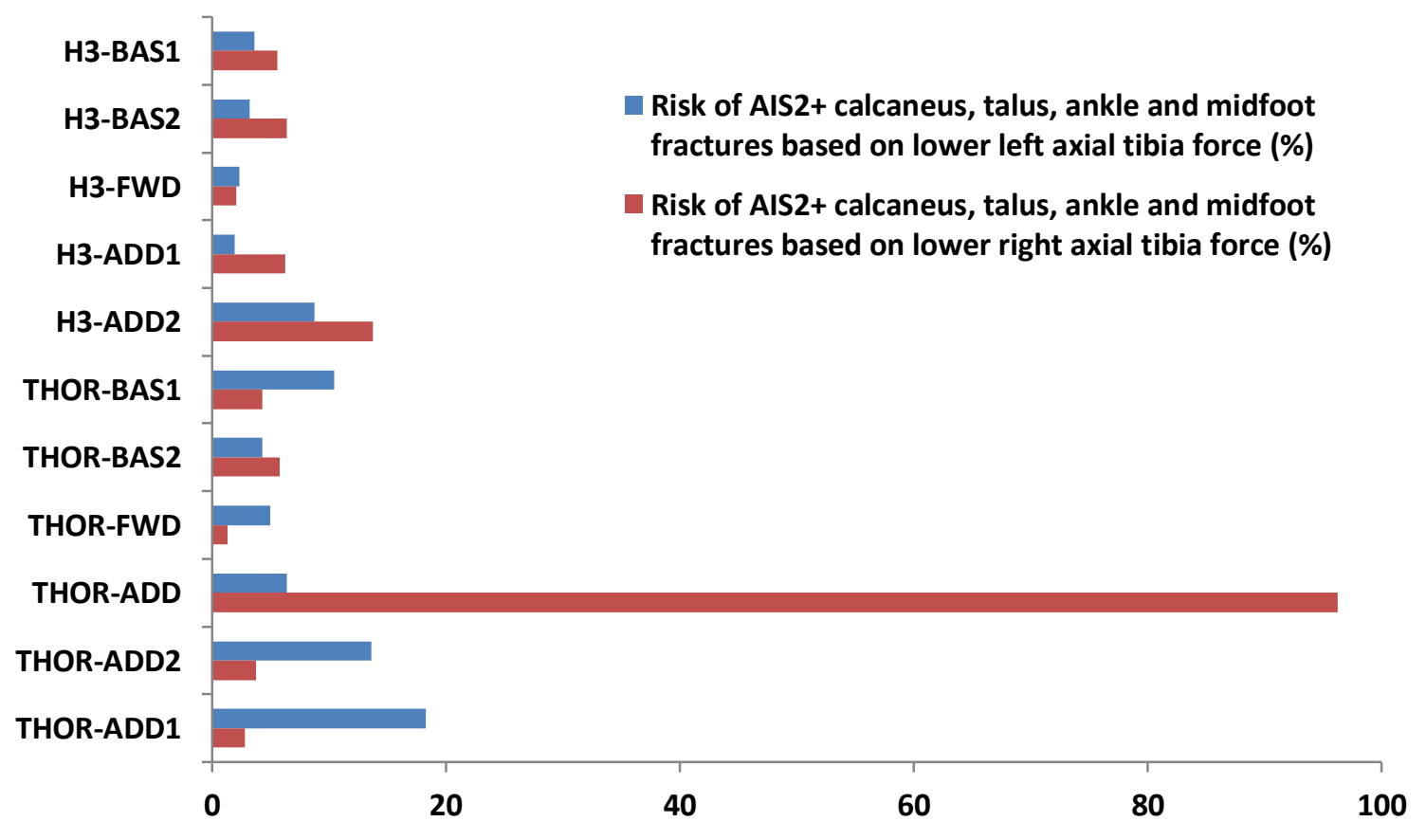


Figure 51. Risk of AIS2+ calcaneus, talus, ankle and mid-foot fractures based on axial tibia force (\%)

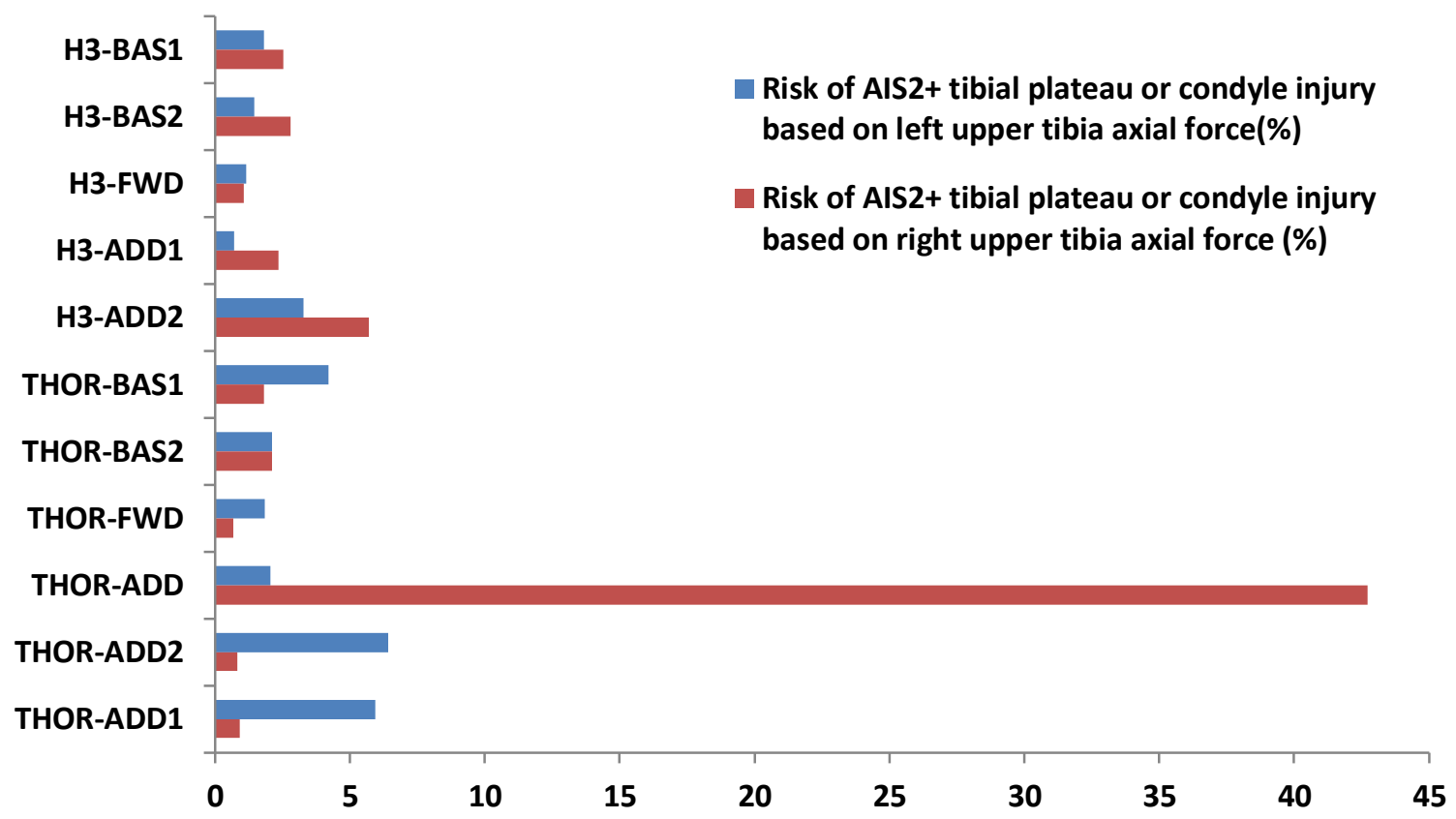

Figure 52. Risk of AIS2+ tibia plateau or condyle injury based on tibia axial force (\%)

The IIHS rating system for the $5^{\text {th }}$ percentile Hybrid-III was applied to the dummy lower limb response and tests data was categorized with reference to the rating boundary values (Table 6).

Table 6. Summary of tests data with IIHS rating system

\begin{tabular}{|c|c|c|c|c|c|c|c|c|c|c|c|}
\hline Num. & 01 & 02 & 03 & 04 & 05 & 06 & 07 & 08 & 09 & 10 & 11 \\
\hline Test & $\begin{array}{l}\text { H3- } \\
\text { BAS1 }\end{array}$ & $\begin{array}{l}\text { H3- } \\
\text { BAS2 }\end{array}$ & H3-FWD & $\begin{array}{c}\mathrm{H} 3- \\
\mathrm{ADD} 1\end{array}$ & $\begin{array}{l}\mathrm{H} 3- \\
\mathrm{ADD2}\end{array}$ & $\begin{array}{l}\text { THOR- } \\
\text { BAS1 }\end{array}$ & $\begin{array}{l}\text { THOR- } \\
\text { BAS2 }\end{array}$ & $\begin{array}{l}\text { THOR- } \\
\text { FWD }\end{array}$ & $\begin{array}{l}\text { THOR- } \\
\text { ADD }\end{array}$ & $\begin{array}{l}\text { THOR- } \\
\text { ADD2 }\end{array}$ & $\begin{array}{l}\text { THOR- } \\
\text { ADD1 }\end{array}$ \\
\hline Tibia Lower Left Fz & -1382 & -1265 & -900 & -711 & -2396 & -2606 & -1581 & -1752 & -2025 & -2928 & -3307 \\
\hline Tibia Lower Right Fz & -1870 & -2036 & -790 & -2009 & -2944 & -1584 & -1914 & -277 & -8404 & -1423 & -1117 \\
\hline Tibia Upper Left Fz & -1480 & -1281 & -1063 & -644 & -2012 & -2237 & -1610 & -1492 & -1590 & -2625 & -2552 \\
\hline Tibia Upper Right Fz & -1771 & -1870 & -998 & -1709 & -2516 & -1479 & -1617 & -602 & -4723 & -783 & -866 \\
\hline Left Femur Fz & -275 & -210 & -205 & -192 & -448 & -336 & -211 & -366 & -306 & -532 & -463 \\
\hline Right Femur Fz & -320 & -334 & -260 & -745 & -684 & -367 & -335 & -234 & -512 & -391 & -247 \\
\hline $\begin{array}{l}\text { Resultant Left Foot } \\
\text { Acceleration }(x, z)\end{array}$ & 58 & 46 & 106 & 44 & 98 & 78 & 55 & 100 & 90 & 149 & 122 \\
\hline $\begin{array}{l}\text { Resultant Right Foot } \\
\text { Acceleration }(x, z)\end{array}$ & 50 & 53 & 52 & 335 & 325 & 61 & 77 & 80 & 355 & 169 & 90 \\
\hline
\end{tabular}




\section{DISCUSSION}

Given the field data findings of increased risk of leg injuries in crashes involving a knee airbag, the tibia index was applied in this study of static knee airbag deployment as an injury criterion to represent lower limb injury risk. It is recognized, however, that $\mathrm{TI}$ can result in inaccurate injury prediction given the geometry and stiffness of Hybrid-III, since the TI values are affected by the geometry of the Hybrid-III dummy, which does not represent the geometry of the human leg in a biofidelic manner (Welbourne et al., 1998; Funk et al., 2004). Due to the bent shape of the instrumented Hybrid-III leg, artifactual bending moments not present in the human leg are recorded at the upper and lower tibia load cells owing to the axial force being applied along a line of action behind the upper tibia load cell, but in front of the lower tibia load cell. The human tibia diaphysis generally bows anteriorly and medially, especially at the proximal tibia (Funk et al., 2004). Therefore, a geometric adjustment developed by Zuby et al. for the tibia moments was applied in the study in an attempt to reduce this confounding factor (Zuby et al., 2001). Given the limited information on curvature of the tibia at the time of development, the THOR-FLx used a straight component for the leg, which, like the Hybrid-III, does not match the human geometry, although the variation between human and dummy anthropometry is much smaller for the THOR-FLx than the Hybrid-III. In addition to geometric issues, the compliance of the belowknee structures must be considered for characterization of axial loads. While geometric adjustment can partially compensate for the leg curvature, the stiff structure of the Hybrid-III frequently overestimates the loads relative to what a human leg would experience. For comparison, the THOR-FLx incorporates axial compliance of the lower leg using a deformable element inserted into the proximal tibia shaft. This element lowers the effective stiffness of the metal column that constitutes the tibia. As a result, the axial forces in THOR-FLx are more comparable to the human response whereas the Hybrid-III generally produces higher axial forces due to its stiff structure. In the deployed knee airbag environment, the Hybrid-III generally showed higher tibia index than THOR-FLx, especially in the upper right tibia region.

All tests involved static deployment of knee airbags in a simplified buck environment. The purpose of the study was to acquire a better assessment of driver lower limb kinematics and forces resulting solely from KAB deployment effects, and to exclude other contributing factors that may come to play in dynamic sled-tests,

including the crash-pulse magnitude, vehicle intrusion levels and onset time, and occupant kinematics during the crash. Real-world crashes would superimpose crash loads and intrusion onto the forces observed with KAB deployment and the effects on injury in a dynamic environment cannot be assessed at this juncture. In addition, pre-impact braking and bracing could influence the occupant motions and forces within the occupant compartment. High incidence of pre-crash bracing has been indicated by skid marks and anticipated reaction time, and in more than two-thirds of occupants in frontal crashes who sustained lower extremity injuries, the occupants were noted to have braced their leg muscles during the impact (Petit et al., 1998; Ore 
et al., 1992). While the driving posture from test H3-ADD2 and THOR-ADD2 attempted to reproduce the braking posture of small female drivers, actual muscle bracing would generate additional loading to the lower limbs. Levels of muscle activation could potentially affect load distribution and injury risks during a frontal crash, and braking could potentially elevate the foot placement and place the tibia position closer to the knee airbag.

Leg abduction as a result of deployment was observed in the tests, with an increased abduction angle occurring when the dummy was translated forward. Increased loading to the upper and lower tibia was observed when left leg was moved inboard and knees moved closer in lateral, creating an initial posture of adduction for both limbs which essentially "trapped" the deploying knee airbag. Video analysis supported the hypothesis that knee airbag could potentially alter the occupant positioning such that legs are repositioned towards stiffer outboard or inboard vehicle structures within the occupant compartment, which may cause higher loads during contact in a crash. Schroeder et al. performed four out-of-position PMHS static deployment tests and one in-position PMHS sled test (Schroeder et al., 2005). High axial compression force in femur and lateral movement of legs was noticed due to abducting and rotational forces in static deployment tests, similar to the leg abduction observed from this study. Although no critical contact injuries occurred in the dynamic test with PMHS seated in normal position, the test sample size was too small to derive a conclusive judgment regarding the knee airbag performance on preventing lower extremity injuries.

In terms of the representativeness of the experiments, there are multiple contributing factors that may account for elevated dummy lower extremity responses in the experiments beyond that observed in production vehicles. Firstly, the mounting brackets and rigid boundary conditions of the instrument panel and floor pan may have prevented energy absorption by the supporting structures. This change from a potential OEM design could also be a factor to cause the airbag to seem more aggressive from an occupant loading point-of-view. Secondly, the use of a rigid flat seat could have influenced the interaction of the dummy and seat and, by extension, the loading of the upper and lower tibia. Perforation of the airbags occurred in three tests, which resulted in relatively less oscillation during the decaying phase of the data time histories, but the implication for peak loads and moments was negligible.

Results from this study indicate that knee airbag deployment alone may produce forces and moments that could result in lower limb trauma. Since this study was conducted with a single KAB design and inflator, in a controllable but simplified vehicle environment, the findings cannot be broadly generalized. Changes of knee airbag deployment characteristics and mounting positions could also potentially result in different responses in the lower limb. Improvements to knee airbag design have been proposed, including reduction of the gasmass during the filling phase of the airbag by using a dual-staged gas inflator, structural improvement of the airbag mounting bracket, and redirection of the airbag gas flow (Sohr et al., 2010). Given the limitations in this 
static deployment test setup, further investigations may be necessary to assess the knee airbag performance for in-position and out-of-position occupants in dynamic events.

\section{CONCLUSIONS}

This study investigated the biomechanics of lower extremities subjected to direct loading by a deploying KAB. Results showed upper tibia index ranged from 0.95 to 1.31 , and 0.78 to 1.21 for baseline tests of HybridIII and THOR-FLx, respectively. Lower tibia index varied from 0.3 to 0.46 (Hybrid-III) and from 0.51 to 0.79 (THOR-FLx). Translating the dummy to the full-forward position resulted in greater abduction of both legs during knee airbag deployment and an increase of tibia index. The highest average TI was recorded with the left foot moved inboard creating an adducted initial position. For baseline tests, highest injury risk of AIS 2+ leg shaft fractures occurred in upper right tibia of Hybrid-III LX (31.15\%) and in upper left tibia of THOR-FLX (51.17\%). The risk of AIS2+ calcaneus, talus, ankle and midfoot fractures ranged from $1.96 \%$ to $18.32 \%$ for left foot, and from $1.32 \%$ to $96.26 \%$ for right foot. The results predicted higher injury risk of tibia shaft fractures than foot and ankle fractures. Lastly, test data were categorized with reference to IIHS injury assessment reference values. The elevated dummy lower extremity response recorded in this study for out-of-position small female occupants suggests that occupant interaction during deployment needs to be a consideration during knee airbag design. 


\section{CHAPTER 4: General Discussion, Conclusion and Future Work}

\subsection{Discussion and Conclusion}

Lower extremity injury is the leading AIS2+ injury for drivers in frontal crashes. While there has been steady improvement in vehicle crash test performance, the risk of lower extremity injury remains virtually unchanged. Additionally, $73 \%$ of the lower limb injuries currently occur at a toe pan intrusion level less than 2 $\mathrm{cm}$.

As one potential injury countermeasure, knee airbags and inflatable knee bolsters have been purportedly designed to mitigate lower limb injuries and to improve overall safety by proving early restraint for the pelvis and lower torso. However, recent studies from real-world cases with knee airbags suggested an increased risk of tibia/fibula and foot/ankle region with knee airbags. This thesis investigated the lower extremity response and injury with knee airbag deployment, and identified the increased injury risk of tibia/fibula and foot/ankle with knee airbag deployment.

This thesis first examined the lower extremity response for drivers in nominal position, under dynamic condition with the presence of an Inflatable Knee Bolster (IKB). A total of seven unbelted FMVSS208 tests and eight belted NCAP tests were performed with a $50^{\text {th }}$ percentile Hybrid-III dummy to investigate the IKB effects on the kinematics of the lower limb. For the unbelted FMVSS208 tests, average tibia index increased by $34.6 \%$ and 33.3\% for tests with IKB on left upper tibia and left lower tibia, respectively, when compared to tests with standard knee bolster. Additionally, the right upper tibia index increased by $20.3 \%$, while the right lower tibia decreased by $26.5 \%$. For FMVSS 208 tests, IKB increased the injury risk of AIS $2+$ tibia shaft fractures by $8.6 \%$, from tests with standard knee bolster( Ave: 11.6\%, 2.0-32.2\%) to tests with IKB (Ave: 20.2\%, 4.1-51.2\%). Similar results were found for the belted NCAP tests with IKB, with the increase in tibia index for left upper, left lower and right upper tibia relative to standard bolster. A 9.1\% increase in the risk of AIS $2+$ tibia shaft fractures was predicted from tests with standard bolster (Ave: 11.8\%, 3.8-37.5\%) to tests with IKB (Ave: 20.9\%,3.1-53.5\%).

The study also investigated the effects of the knee airbag on the lower limb injury risk for out-of-position drivers. A total of 11 static knee airbag deployment tests were performed with a $5^{\text {th }}$ percentile female HybridIII dummy outfitted with either the original Hybrid-III lower extremities or the $5^{\text {th }}$ percentile THOR-FLx. Baseline tests were performed with FMVSS 208 seating specifications, and a design of experiment for out-of-position conditions was developed with multiple factors including knee-to-instrument panel distance, knee-to-knee distance, and foot placement. The results indicated a $40.6 \%$ increase in risk of AIS2+ tibia shaft fractures for out-of-position drivers relative to in-position drivers. 
This thesis indicated that inflatable knee bolsters could increase the likelihood of drivers sustaining tibia/fibula injuries in frontal crashes. In addition, the dummy lower extremity responses recorded in static deployment tests suggest a high risk of lower extremity injury for both in-position and out-of-position small female drivers during knee airbag deployment.

This thesis utilized a production inflatable knee bolster as a representative example to evaluate the lower limb response in the standardized crash test conditions, and elucidated the adverse effect IKB imposed on the lower extremity during the deployment stage. Studies from the dynamic tests suggested that the increase of tibia index with IKB was directly induced from the increase in tibia bending moment during the IKB deployment. The study also covered the assessment of lower limb injury risk for out-of-position drivers, with a productionlike knee airbag and simplified test boundary conditions to represent the worst-case scenario. Higher injury risk of tibia shaft fracture was observed from the deployment tests for out-of-position drivers compared to drivers with nominal posture, which showed the detrimental effect knee airbags could impose on the lower limb.

Based on the findings of this thesis, the goal of this research was achieved. The proposed null hypothesis was rejected with the acceptance of the alternative hypothesis $H_{1}$ : Between standard knee bolsters and knee airbags, differences exist in injury risk of the tibia/fibula and foot/ankle regions for drivers in frontal motor vehicle crashes. More specifically, the results revealed that this knee airbag increased the injury risk of the tibia/fibula and foot/ankle regions for drivers in frontal crashes in the specific FMVSS208 and NCAP test conditions.

\subsection{Limitation and Future Work}

There are several limitations in current study, which could be summarized as follows.

1) For the dynamic FMVSS 208 and NCAP tests, all the tests were performed with the Hybrid III 50th percentile dummy, which was different from the human lower limb due to its design and bended shape. While geometric adjustment applied in the study accounted for this variation from human lower extremity, the stiff structure of Hybrid III ATD inevitably lacked the tibia compliance and over-estimated the tibia loads compared to the response of human legs. Future study with the advanced THOR dummy could provide a more biofidelic response, and tests with PMHS could indicate the injuries directly rather than predicting an injury risk.

2) The test repeatability could have been improved. For dynamic tests, inter-test variation was observed for tests in the same condition. This could be the results of multiple contributing factors, including the variation from dummy positioning and IKB mounting, the individual variability of deployed inflatable knee bolsters and 
unexpected hardware malfunction during the test (i.e., IKB late deployment). Static out-of-position tests had a better repeatability in baseline tests, but future studies could be done with higher precision.

3) This study utilized a single inflatable knee bolster design for dynamic tests, and a production-like knee airbag for out-of-position static deployment tests. Given the multitude of knee airbag designs, the difference in inflator pressure, airbag geometry, mounting positon and deployment shape could all lead to difference in the resulting lower extremity responses. Additionally, the lower extremity response from the sled tests was partially dependent on the sled pulse input. Until more tests are performed with different types of knee airbags, current findings on knee airbag effects could not be generalized.

Regardless of these limitations, current study was one of the very few publications that provided an indepth examination of the lower extremity response and injury with knee airbag deployment. Results from this thesis indicated the need for improved knee airbag design to better protect the lower extremity, and it is anticipated that knee airbags could provide overall occupant safety without sacrificing the tibia/fibula and foot/ankle region during the transition from controversial to commonplace. 


\section{REFERENCES}

AAAM, 1990. The abbreviated injury scale-1990 revision (AIS 90). Published report, Association for the Advancement of Automotive Medicine.

Bingley, L., Cross, G., Morris, R., 2005. Determination of real world occupant postures by photo studies to aid smart restraint development. Paper 05-0319, International Technical Conference on the Enhanced Safety of Vehicles (ESV).

Bose, D., Crandall, J.R., Untaroiu, C.D., Maslen, E.H., 2010. Influence of pre-collision occupant parameters on injury outcome in a frontal collision. Accident Analysis and Prevention, 42(4): 1398-1407.

Crandall, J.R., Martin, P.G., Bass, C.R., Pilkey, W.D., Dischinger, P.C., Burgess, A.R., O’Quinn, T.D., Schmidhauser, C.B., 1996. Foot and ankle injury: the roles of driver anthropometry, footwear, and pedal controls. 40th Annual Proceedings of the Association for the Advancement of Automotive Medicine, pp. 1-18.

Crandall, J.R., 2014. Driver lower extremity response and injury with knee airbag deployment, University of Virginia/INOVA CIREN center. 2014 CIREN Annual Meeting, National Highway Traffic Safety Administration, Charlottesville, VA.

Crandall, J.R., Martin, P.G., 1997. Lower limb injuries sustained in crashes and corresponding biomechanical research. International Symposium on Real World Crash Injury Research, Leicestershire, UK.

Cushion Restraint Device, 1997. Seat cushion restraint system. US Patent US5695242 A. http://www.google.com/patents/US5695242 (date assessed: 05/02/2015).

Culver, C., 1979. Factors influencing knee restraint. Paper 790322, Society of Automotive Engineers (SAE).

Dischinger, P.C., 1996. The risk of ankle/foot fractures among women drivers. Proceedings of the $2^{\text {nd }}$ National Conference on Women's Travel Issues, Baltimore, MD. Chapter 28, 531-536.

Faure, J., Couturier, S., Page, Y., 2007. The benefits of double pretension in decreasing knees \& lower legs injuries in frontal impacts. $20^{\text {th }}$ International Technical Conference on the Enhanced Safety of Vehicles (ESV), Lyon, France.

Funk, J.R., Rudd, R.W., Kerrigan, J.R., Crandall, J.R., 2004. The effect of tibial curvature and fibular loading on the tibia index. Traffic Injury Prevention, 5(2): 164-172.

Haland, Y., Hjerpe, E., Lovsund, P., 1998. An inflatable carpet to reduce the loading of the lower extremities evaluation by a new sled test method with toe pan intrusion. $16^{\text {th }}$ International Technical Conference on the Enhanced Safety of Vehicles, Windsor, Ontario.

Hault-Dubrulle, A., Robache, F., Pacaux, M.P., Morvan, H., 2011. Determination of pre-impact occupant postures and analysis of consequences on injury outcome. Part I: A driving simulator study. Accident Analysis \& Prevention 43 (1), 66-74.

Hong, S., Jeong, H., Cho, B., Kim, I., 2007. A study on invisible knee airbag cushion shape design using DOE method. $20^{\text {th }}$ International Technical Conference on the Enhanced Safety of Vehicles, Lyon, France. 
Ivarsson, J., Manaswi, A., Genovese, D., Crandall, J., Hurwitz, S., Burke, C., Fakhry, S., 2008. Site, type, and local mechanism of tibial shaft fractures in drivers in frontal automobile crashes. Forensic Science International, Vol. 175, Issue 2-3, Pages 186-192.

Injury assessment reference values (IARV) of $5^{\text {th }}$ dummy testing from Insurance Institute for Highway Safety, 2014. http://www.iihs.org/iihs/ratings/ratings-info/frontal-crash-tests (date assessed: 11/20/2014).

Jenkins, J, Ridella S, Ham S., 2002. Development of an inflatable knee bolster by using MADYMO and DOE. $9^{\text {th }}$ International MADYMO User's Conference, Italy.

Kato, K., Fukaya, Y., Muramatsu, M., Fujimoto, H., 2007. Development of driver side SRS knee airbag. Internal Mitsubishi Technical Review, No.19.

Kuppa, S., Wang, J., Haffner, M., Eppinger, R., 2001. Lower extremity injuries and associated injury criteria. $17^{\text {th }}$ Conference on the Enhanced Safety of Vehicles, Paper No. 457, Amsterdam, Netherlands.

Leport, T., Baudrit, P., Petit, P., Trosseille, X., Vallancien, G, 2009. Evaluation of the risk of injury caused by a knee airbag in out-of-position. Proceedings of the $21^{\text {st }}$ International Technical Conference on the Enhanced Safety of Vehicles (ESV), Stuttgart, Germany.

Lewandowski J., 2007. FMVSS 208 technical report. Report Number 208-MGA-2007-005.

Malczyk A, Kalliske I., 2003. Knee airbag effects on 5\% percentile female. Proceedings of the International Conference on the Biomechanics of Impact (IRCOBI), Lisbon, Portugal.

Masuda, M., 2014. Knee airbag out-of-position issues. Presentation at ISO Committee Meeting, ISO TC22 SC10 WG3 N292, Toyota Motor Corporation, Japan.

McCarthy, M., Couper, G., 2005, Restraint feature fit and crash test data, Report from proposed reduction of car crash injuries through improved smart restraint. http://www.prismproject.com (date assessed: 05/20/2015).

Mertz, H., 1993. Anthropomorphic test devices, accident injury, biomechanics, and prevention. Springer New York, DOI: 10.1007/978-1-4757-2264-2_4.

Morris, R., Cross, G., Bingley, L., 2004. Improved understanding of passenger behavior during pre-impact events to aid smart restraint development. Report from PRISM project: http://www.prismproject.com (data assessed: 06/28/2015).

Morris, R., Cross G., 2005. Improved understanding of passenger behavior during pre-impact events to aid smart restraint development. $19^{\text {th }}$ International Technical Conference on the Enhanced Safety Vehicles, USA. National Automotive Sampling System-Crashworthiness Data System, 2010. NASS-CDS coding and editing manual, U.S. Department of Transportation.

Nie, B., Ye, X., Riley, P., Crandall, J., Panzer, M., 2015. Investigation of active muscle response on the occupantknee airbag interaction in frontal impacts. Proceedings of International Research Council on the Biomechanics of Injury, Lyon, France. 
Ohachi, J., Masuda, M., Katsumata, S., Kanno, Y., 2012. Consideration of protection effects with knee airbag by the simulation of frontal impact and the analysis of traffic accident data. JSAE paper number 20125279.

Ore, L., Tanner, B., Pritz, H., 1992. Design requirements and specifications: dummy lower extremity development task. Event report from National Highway for Transportation Safety Administration.

Patel, V., Griffin, R., Eberhardt, W., McGwin, G., 2013. The association between knee airbag deployment and knee-thigh-hip fracture injury risk in motor vehicle collisions: a matched cohort study. Accident Analysis and Prevention, doi: 10.1016/j.aap.2012.07.023.

Petit, P., Portier, L., Trosseille, X., 1998. Rigid body model of the Hybrid-III dummy lower limb including muscle tendon under car crash conditions. Proceedings of IRCOBI conference, pp 173-188.

Pickrell, T. M., \& Choi, E. H., 2015. Seat belt use in 2014- overall results. Traffic safety facts research note. Report No. DOT HS 812 113. Washington, DC: National Highway Traffic Safety Administration.

Reed, M.P., Ebert, S.M., Hallman, J.J., 2013. Effects of driver characteristics on seat belt fit. Stapp Car Crash Journal, 57(2013-02): 43-57.

Roychoudhury, R.S., Best, M.J., Conlee, J.K., 2004. Blow-molded plastic active knee bolsters. SAE World Congress \& Exhibition. Paper 2004-01-0844.

Rudd, R., Crandall, J., Hjerpe, E., Haland, Y., 2001. Evaluation of lower limb injury mitigation from inflatable carpet in sled tests with intrusion using the Thor LX. Paper number $149,17^{\text {th }}$ International Technical Conference on the Enhanced Safety of Vehicles, Amsterdam, Netherlands.

Rudd, R., Shaw, G., Crandall, J., 2003. Fifth percentile female Hybrid-III and THOR-FLx performance in sled tests with toe pan intrusion. Proceedings of $18^{\text {th }}$ International technical conference on the enhanced safety of vehicles, paper number 491, Nagoya, Japan.

SAE J211-1 standard: instrumentation for impact test, Part 1-electronic instrumentation, 1995. Society of Automotive Engineers, Inc.

Schneider, L. W., Robbins, D. H., Pflüg, M. A., Snyder, R. G., 1983. Development of anthropometrically based design specifications for an advanced adult anthropomorphic dummy family. Vol 1 (Technical Report). University of Michigan Transportation Research Institute.

Schroeder, G., Bosch, U., 2005. Is the knee bag save in out of position situations? $1^{\text {st }}$ International Conference on ESAR “Expert Symposium on Accident Research", 2005, S. 189-191.

Shams, T., Beach, D., White, R., Rangarajan, N., Haffner, M., Eppinger,R., Pritz, H., Kuppa,S., Beebe, M., 1999. Development and design of the THOR-LX: The THOR lower extremity. Proceedings of the $43^{\text {rd }}$ Stapp Car Crash Conference, Paper 99SC09, Warrendale, PA.

Shams, T., Beach, D., Huang, T., Rangarajan, N., 2002. Development of THOR-FLx: a biofidelic lower extremity for use with 5th percentile female crash test dummies. Stapp Car Crash Journal, Vol. 46, pp. 267-283. 
Sohr, S., Heym, A., 2010. Benefit of adaptive occupant restraint systems with focus on the new US-NCAP rating requirements. Technical paper of FISITA world automotive congress, Budapest, Hungary.

Swanson, J., Rockwell, T., Beuse, N., Summers, L., Summers, S., Park, B., 2003. Evaluation of stiffness measures from the U.S. new car assessment program. Proceedings of the $18^{\text {th }}$ Conference on the Enhanced Safety of Vehicles. Paper No. 527.

Weaver, A., Loftis, L., Stitzel, J., 2013. Investigation of the safety effects of knee bolster airbag deployment in similar real world crash comparison. Traffic Injury Prevention, 14(2):168-80.

Wellbourne, E.R., Shewchenko, N., 1998. Improved measures of foot ankle injury risk from the Hybrid III tibia. Proceedings of the $16^{\text {th }}$ International Technical Conference on the Enhanced Safety of Vehicles, 1618-26. Washington, DC: National Highway Traffic Safety Administration.

Ye, X., Panzer, M., Shaw, G., Crandall, J., 2014. Driver lower extremity response to out of position knee airbag deployment. IRC-14-27, Proceedings of International Research Council on the Biomechanics of Injury, Berlin, Germany.

Ye, X., Crandall, J., Forbes, A., Bose, D., Hurwitz, S., Funk, J., Shaw, G., Sochor, M., Poplin, G., Freeth, R., Rizzo, A., Rudd, R., Scarboro, M., 2014. Case series analysis of hind-foot injuries sustained by drivers in motor vehicle crashes. Proceedings of International Conference of Crashworthiness, Kuching, Malaysia.

Ye, X., Poplin, G., Bose, D., Forbes, A., Hurwitz, S., Shaw, G., Crandall, J., 2015. Analysis of crash parameters and driver characteristics associated with lower limb injury, Accident Analysis \&Prevention, 83(2015)37-46.

Zuby, D.S., Nolan, J.M., Sherwood, C.P., 2001. Effect of Hybrid III leg geometry on upper tibia bending moments. SAE paper 2001-01-0169, Detroit, Michigan. 


\section{APPENDICES}

\section{Appendix A. Tests data from FMVSS208 and NCAP tests}

\section{FMVSS208 Tests Data}
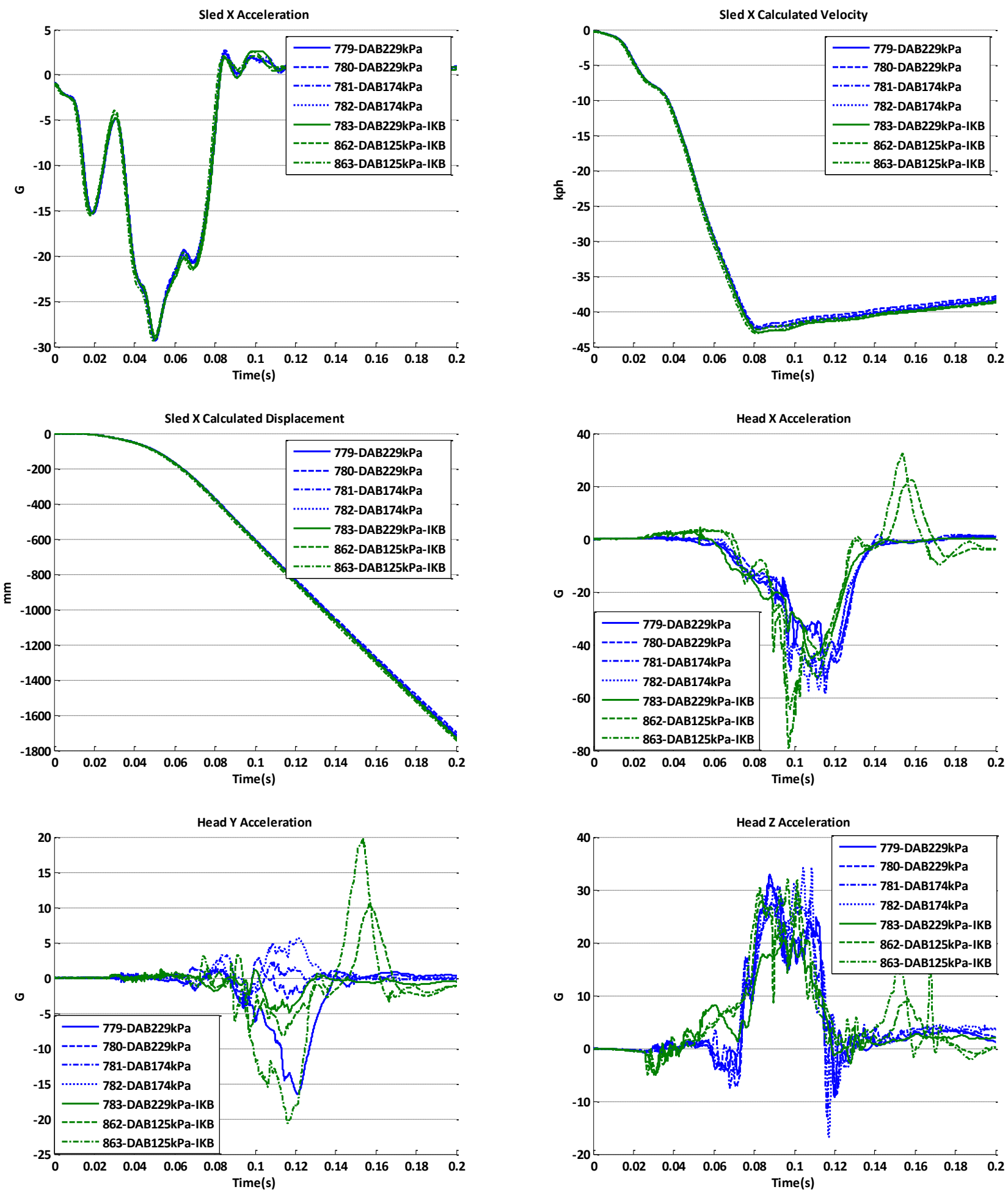

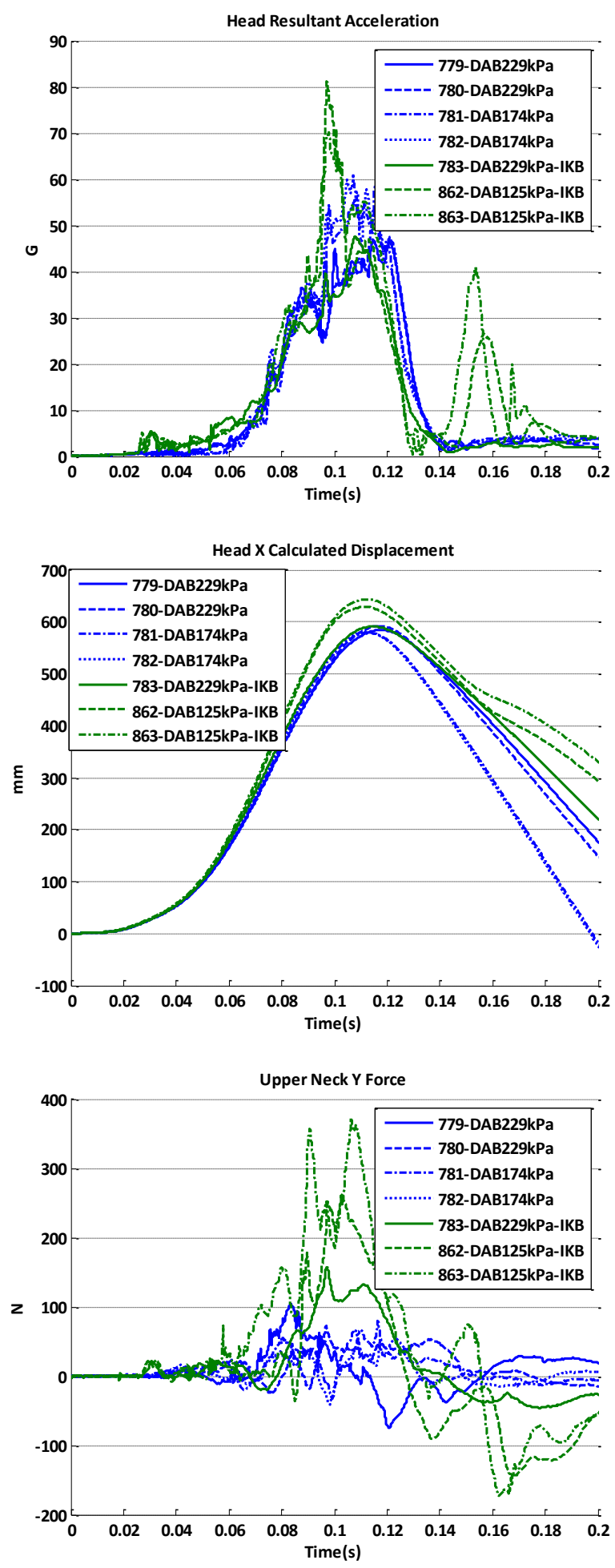
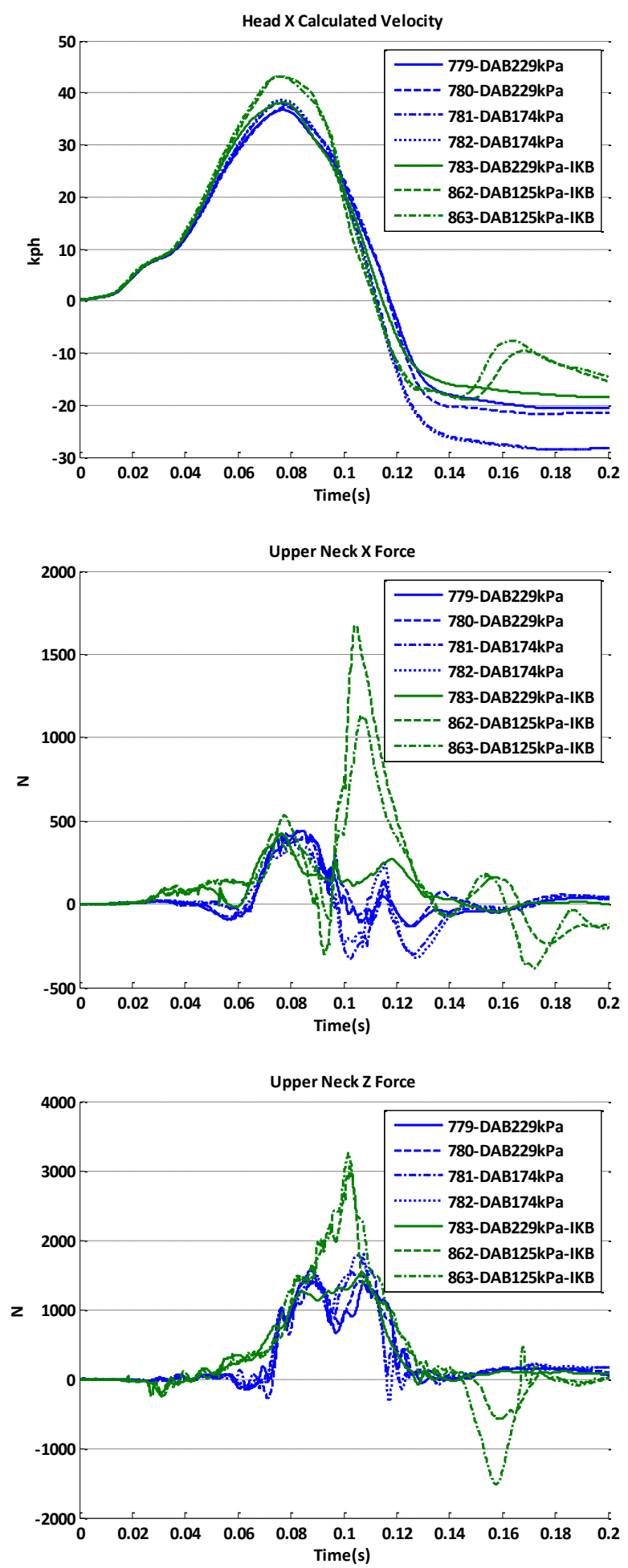

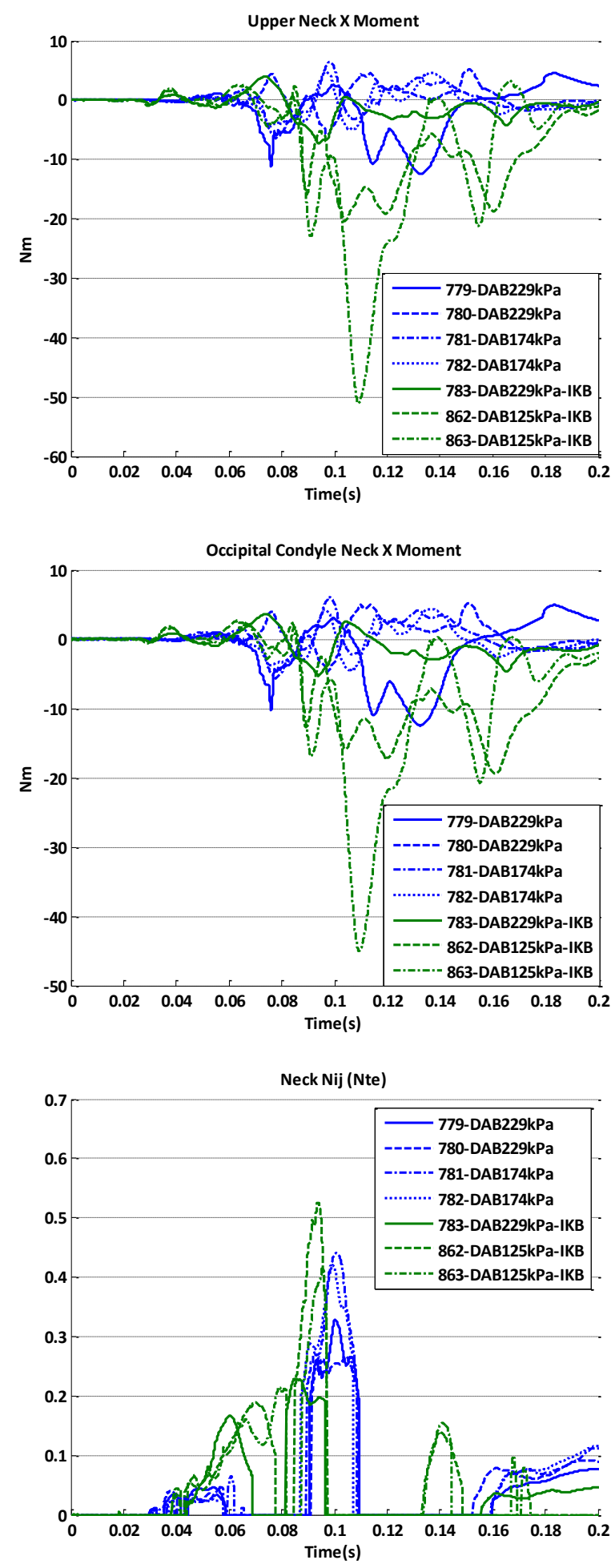
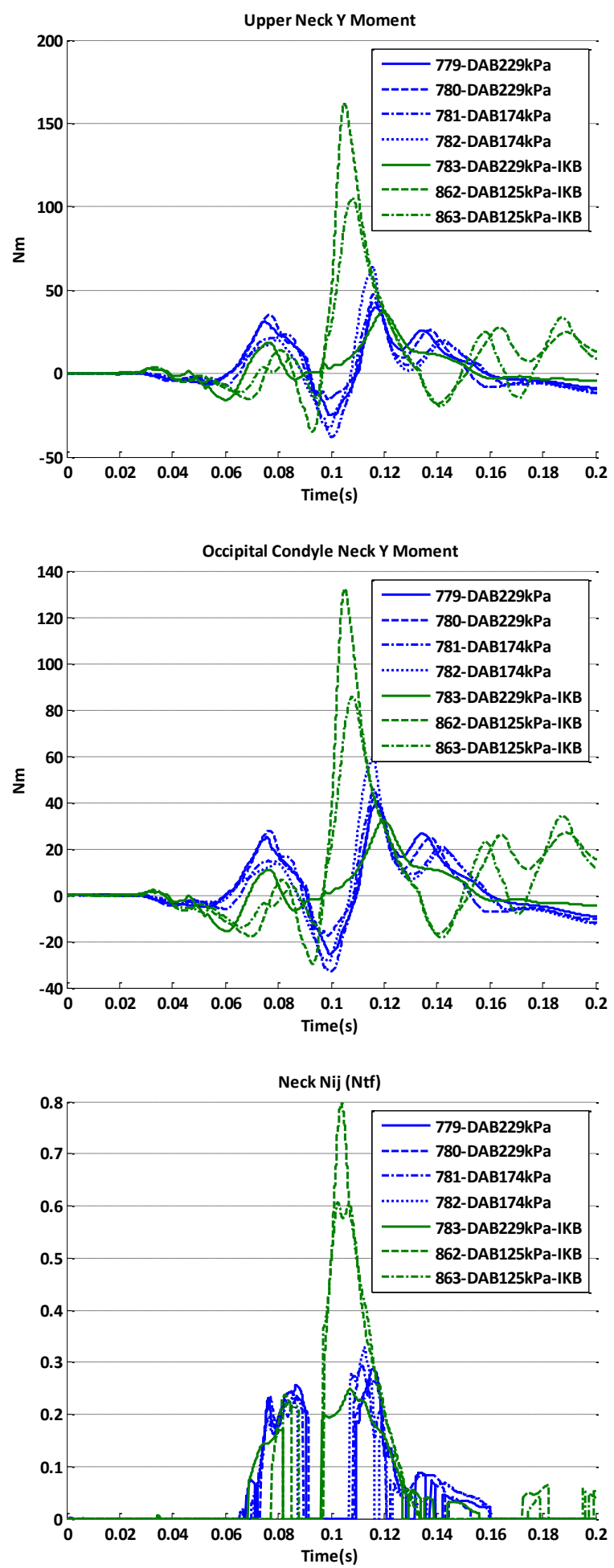

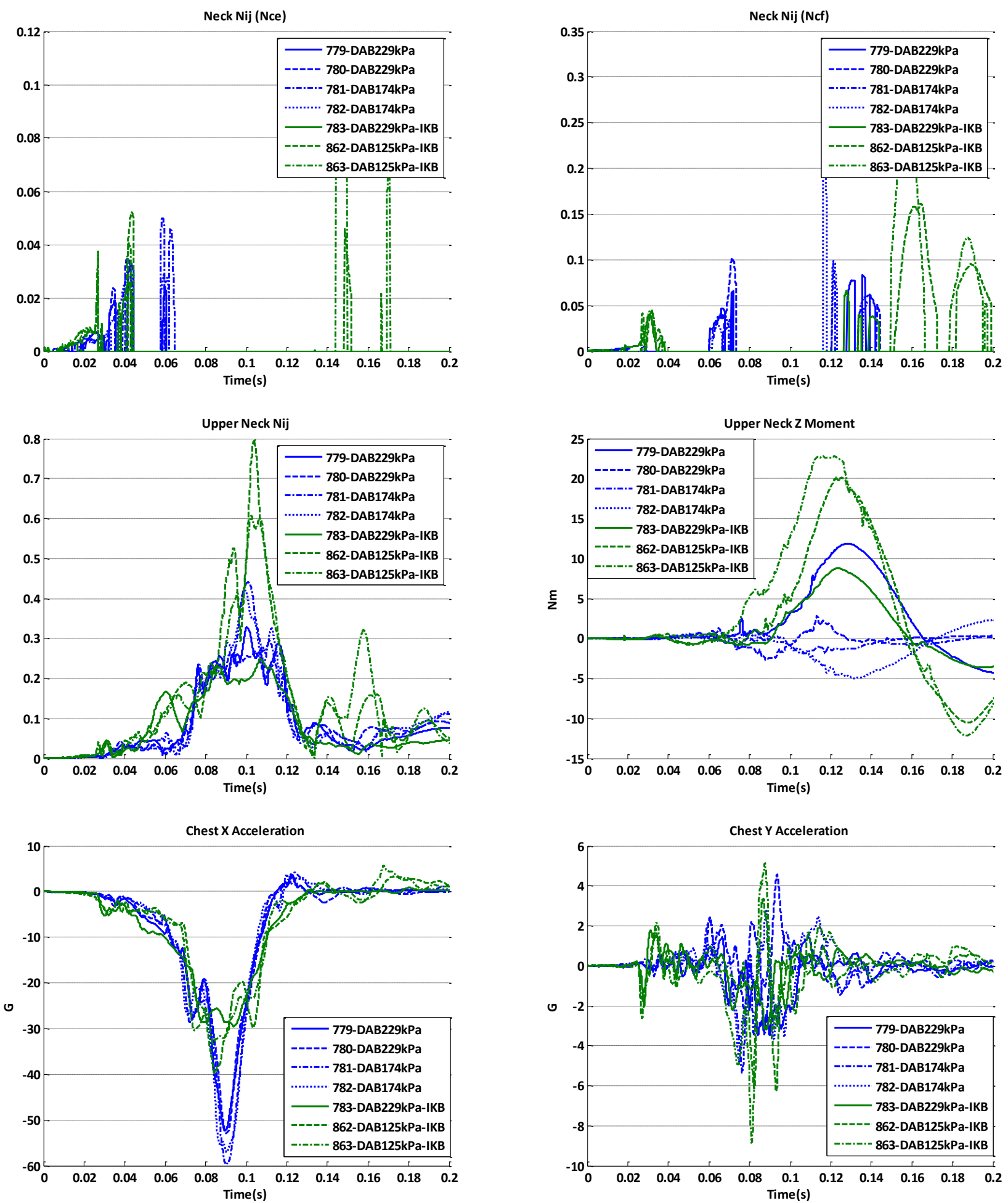

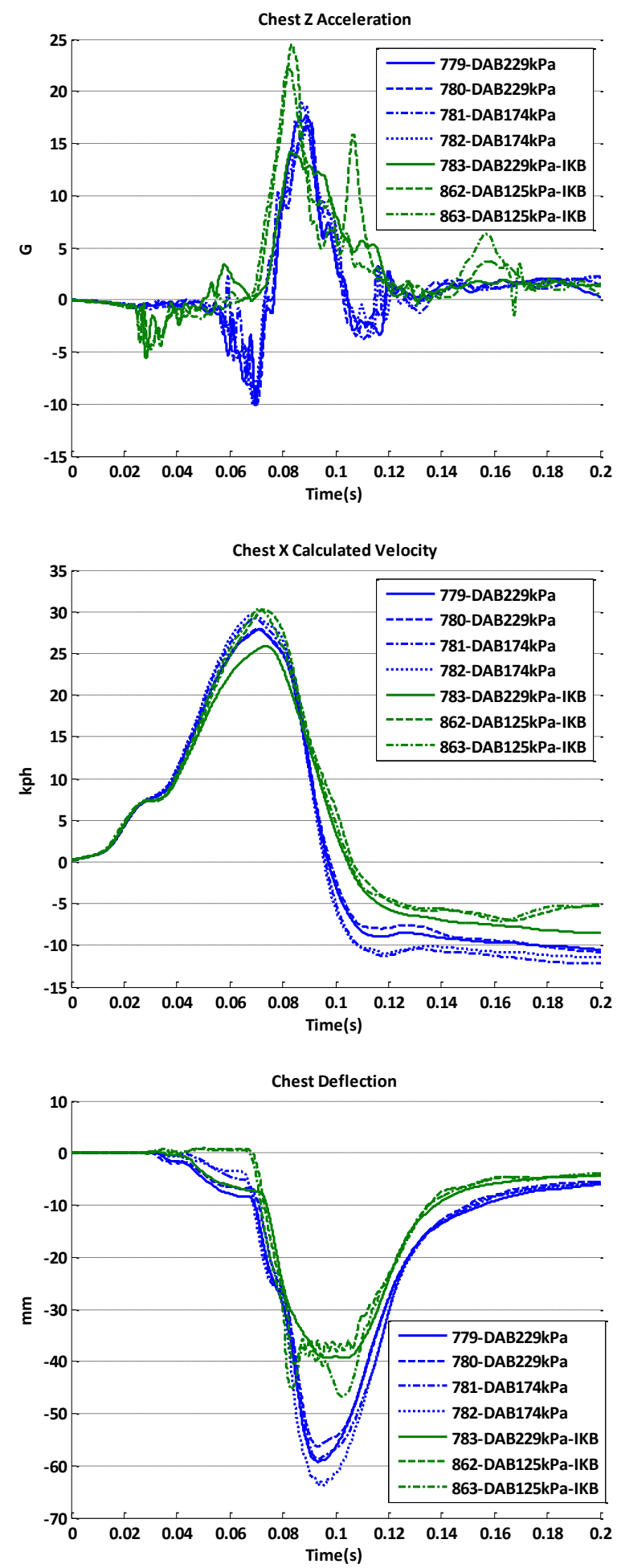
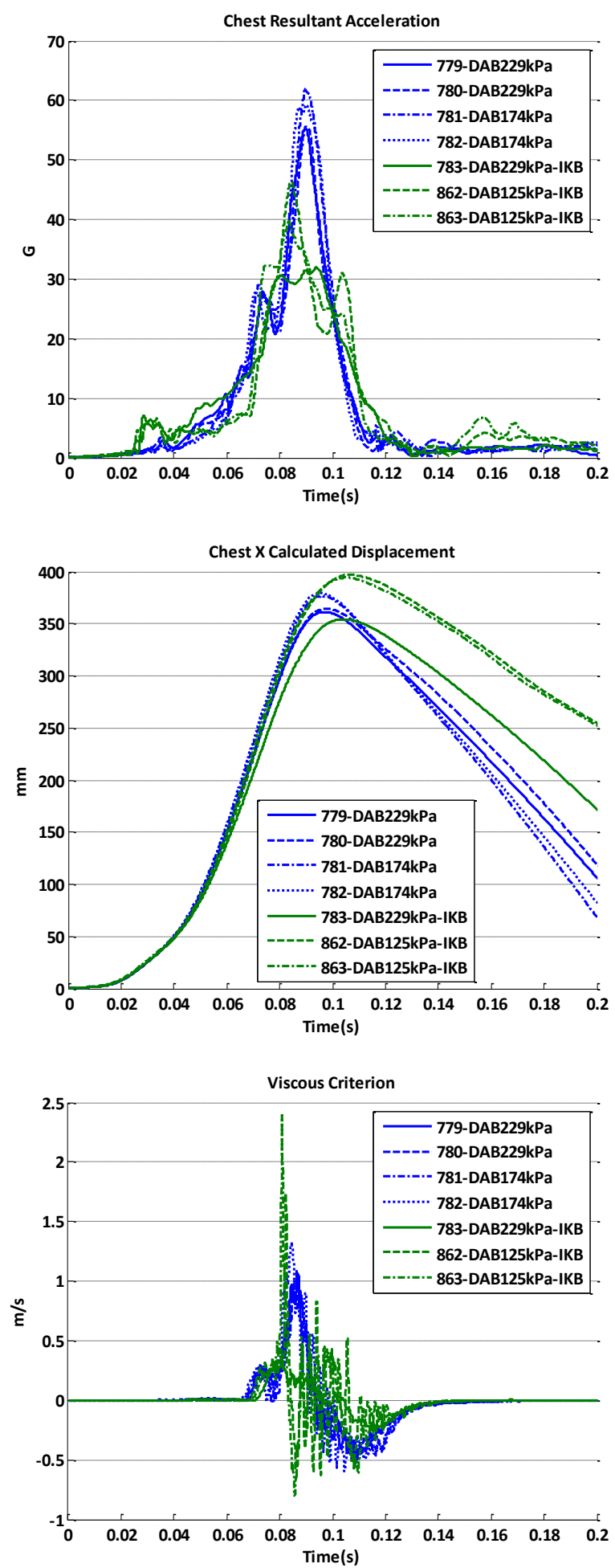

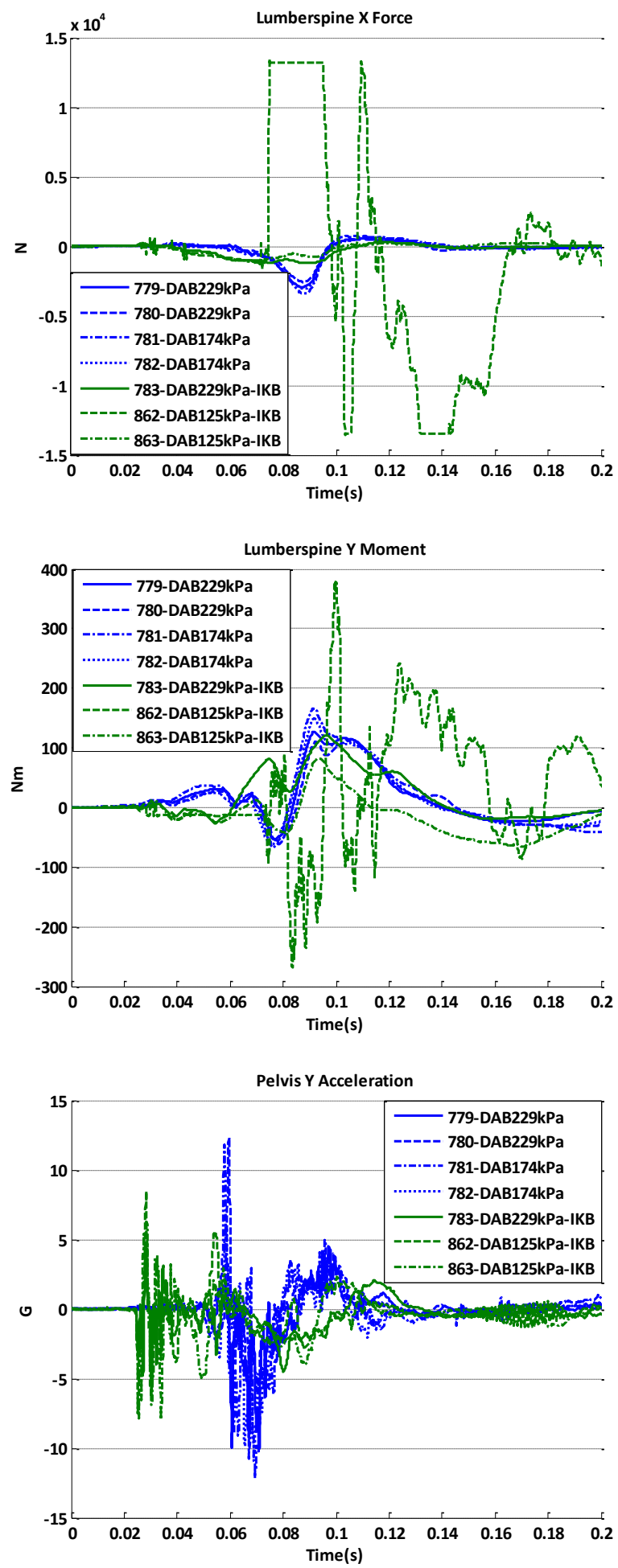
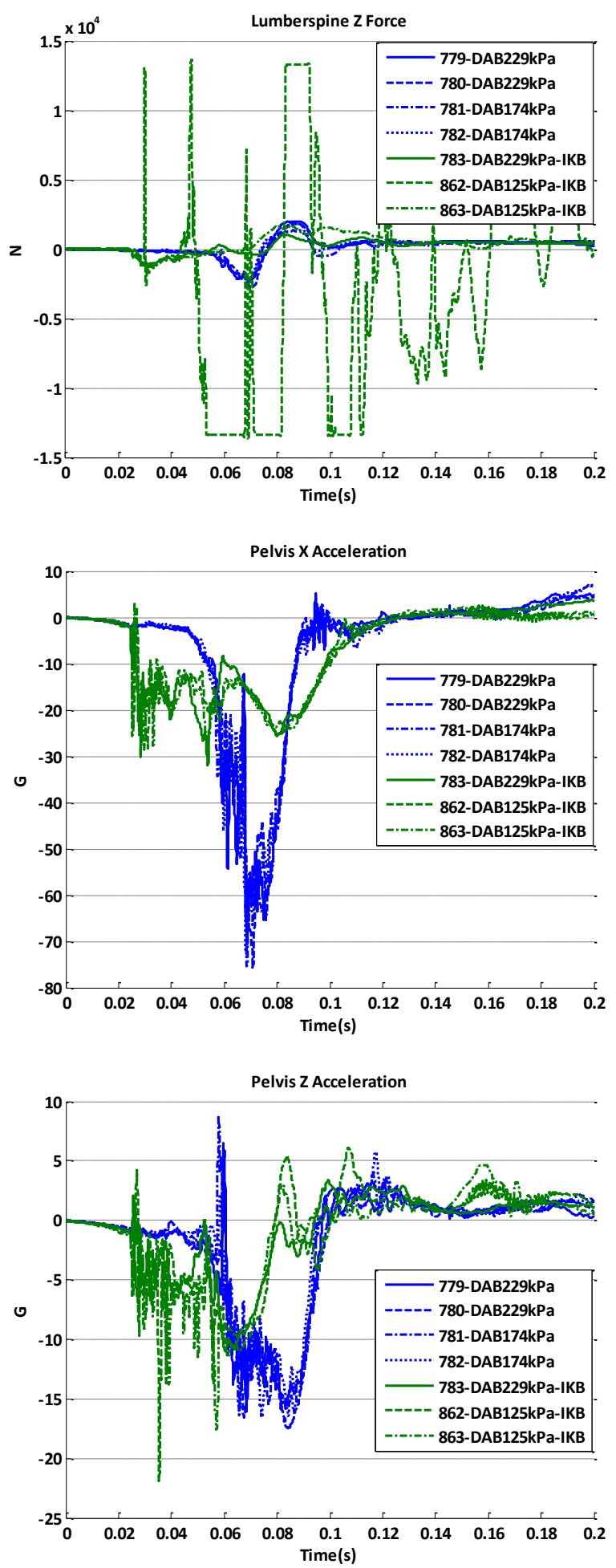

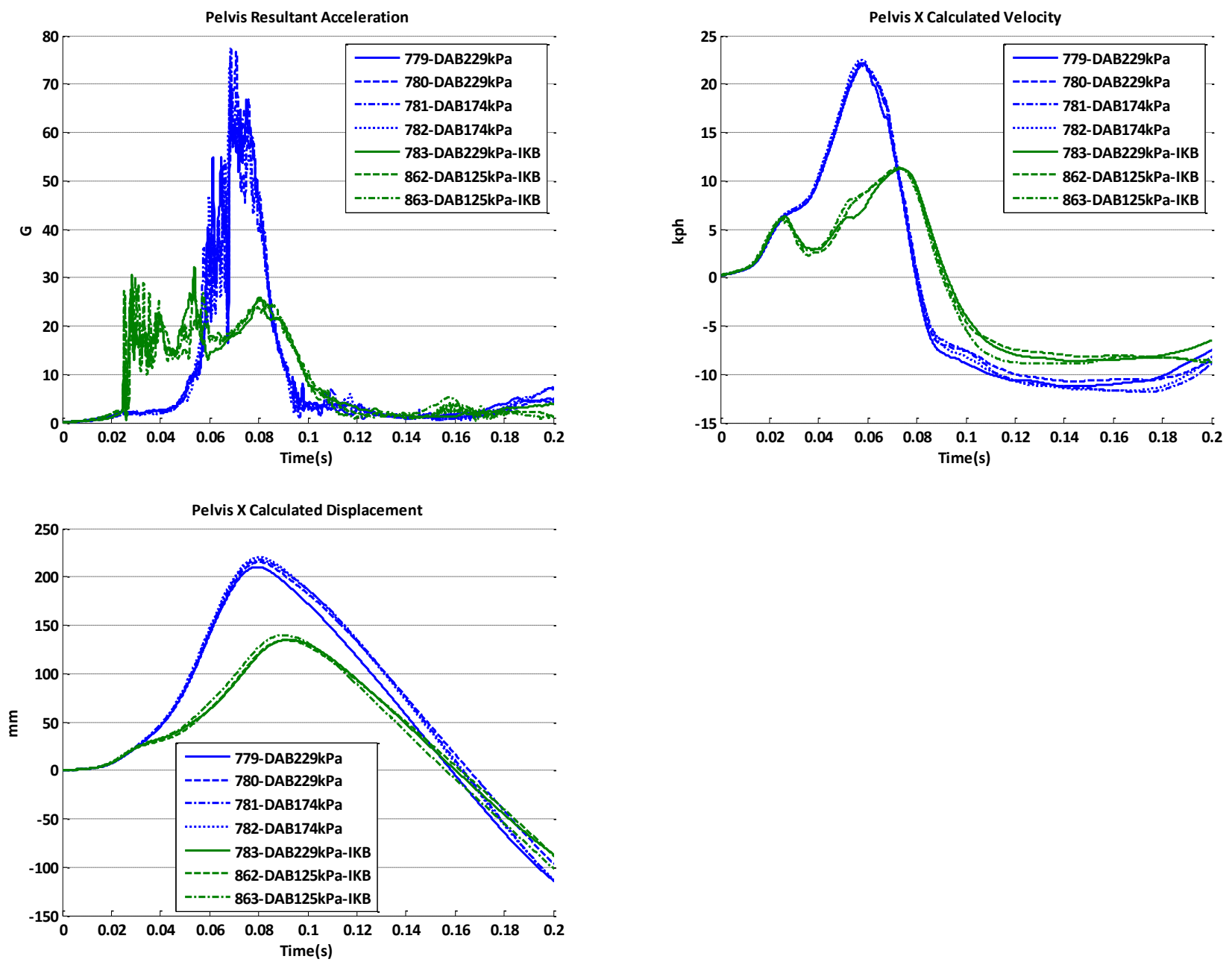

\section{NCAP Tests Data}
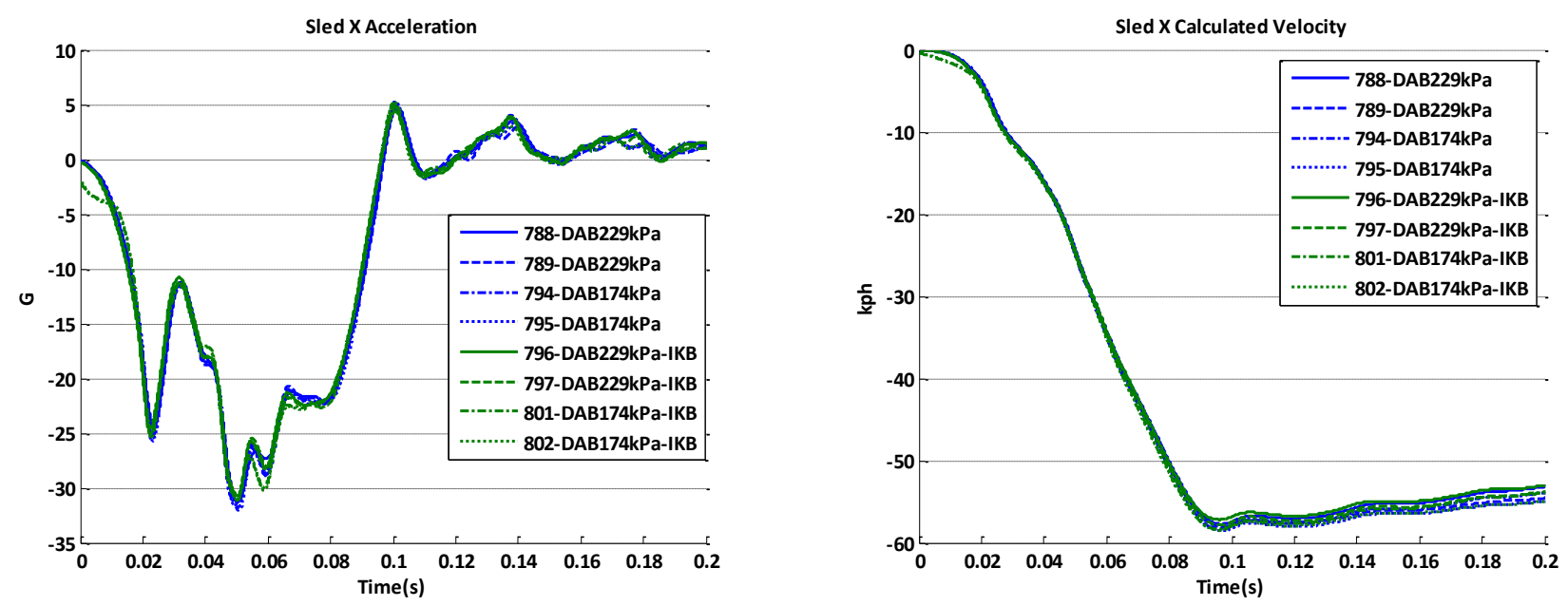

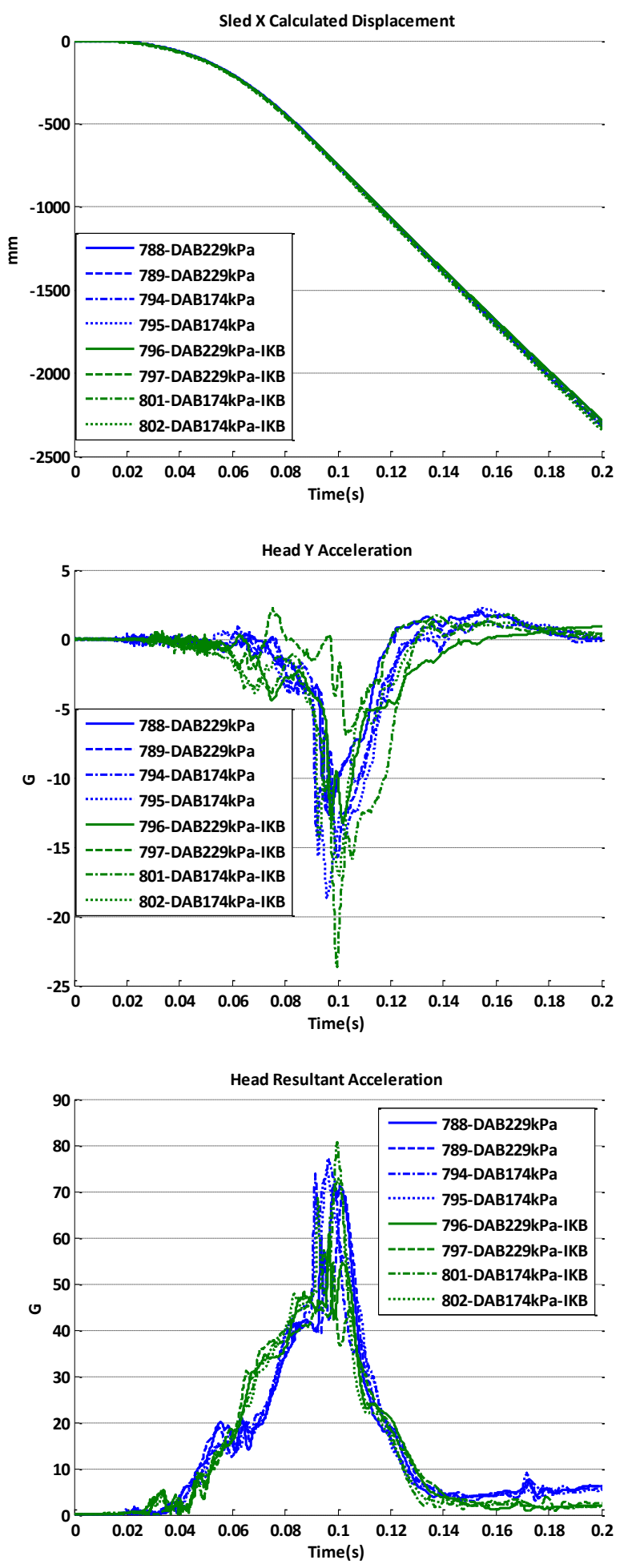
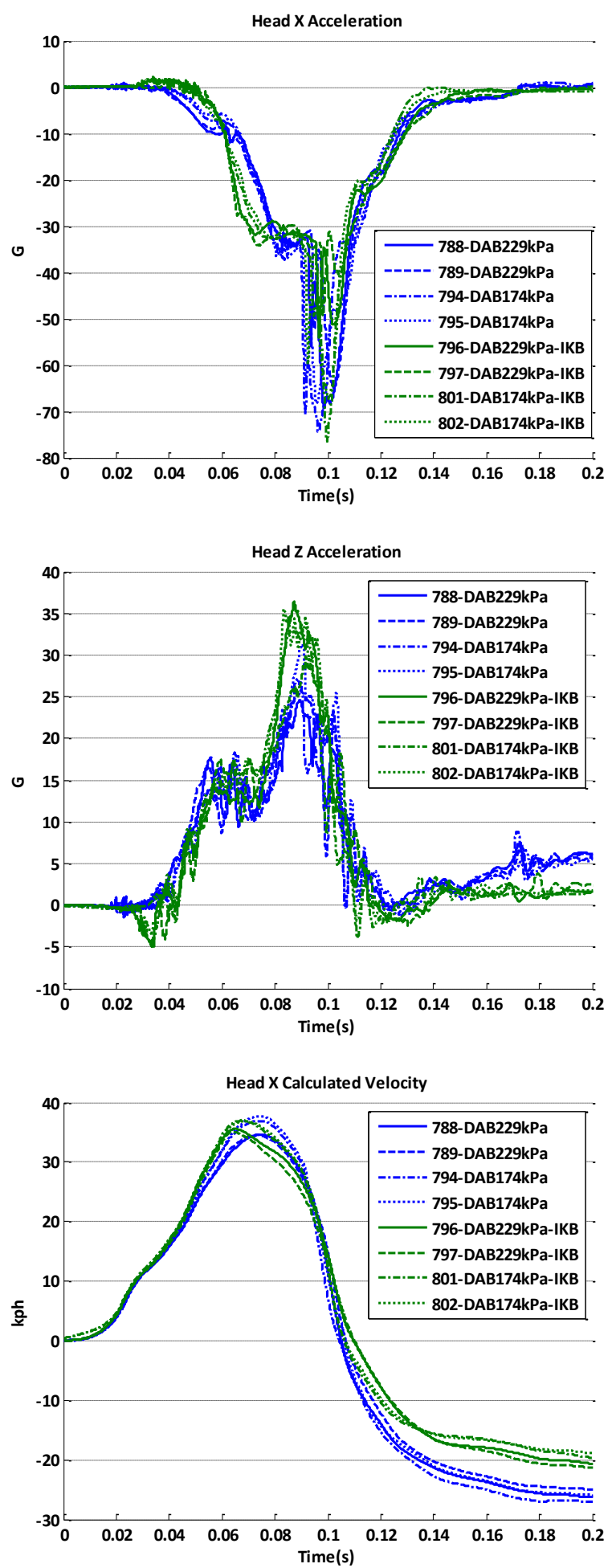

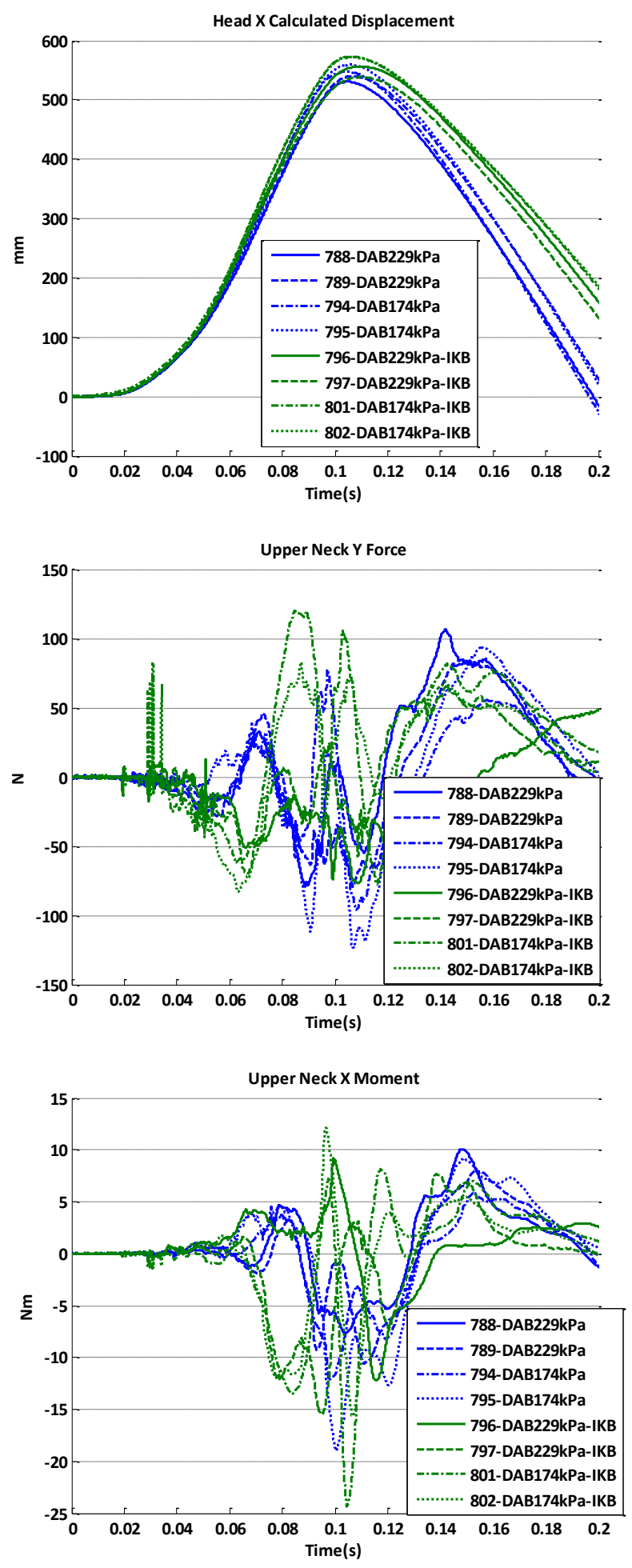
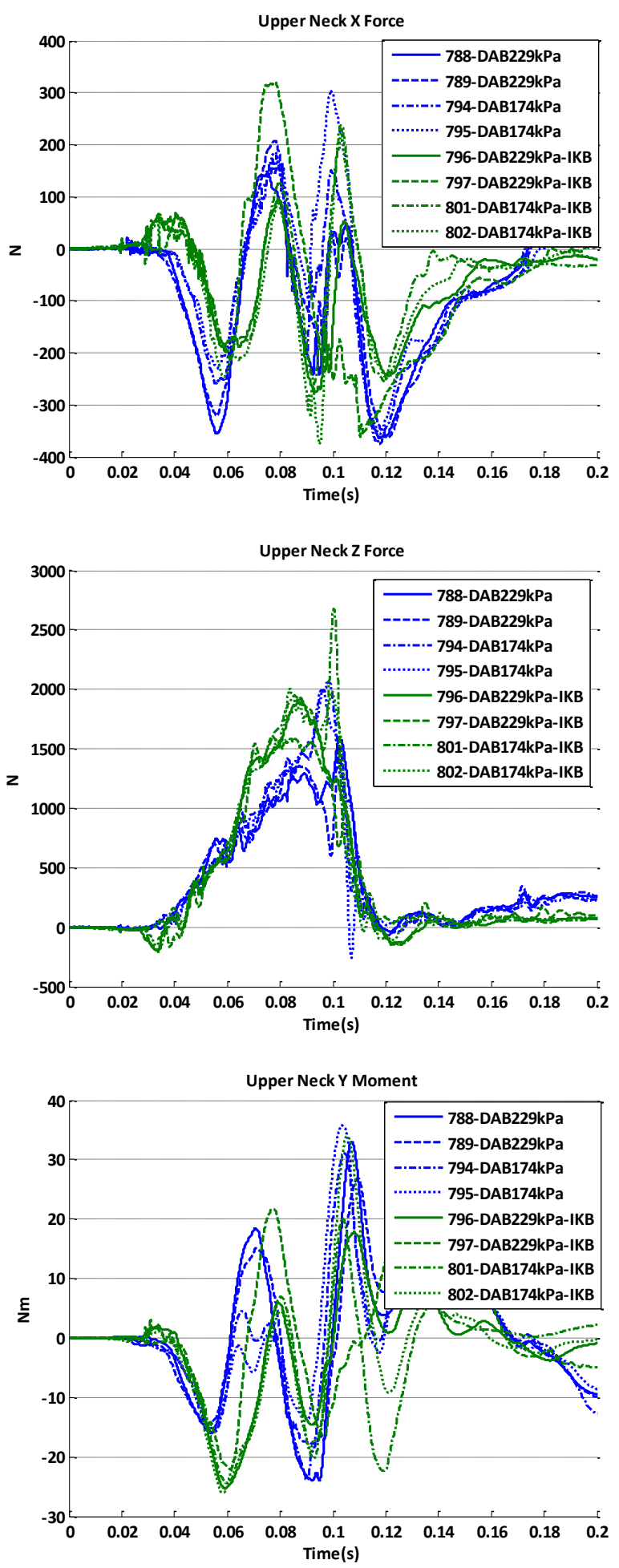

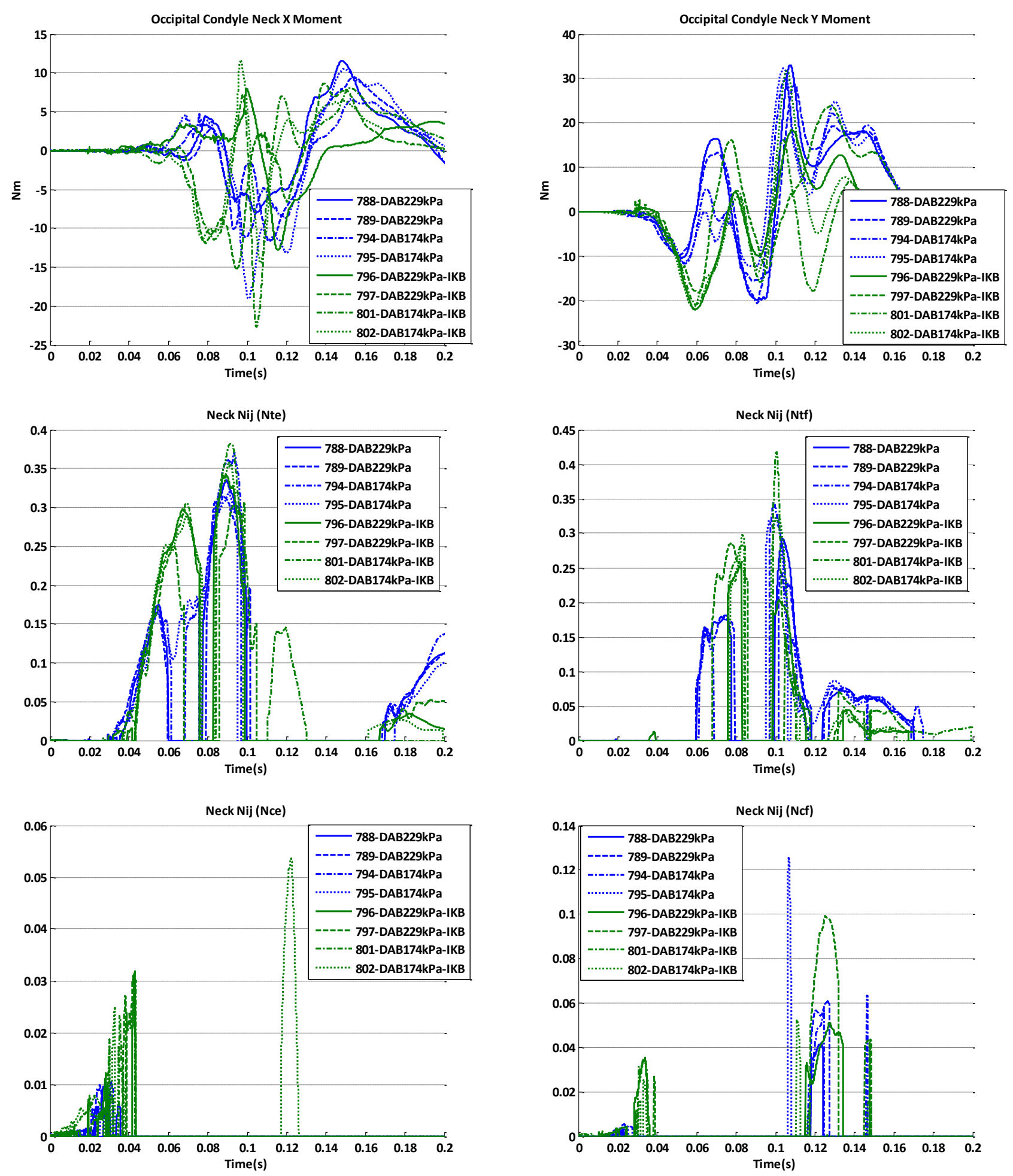

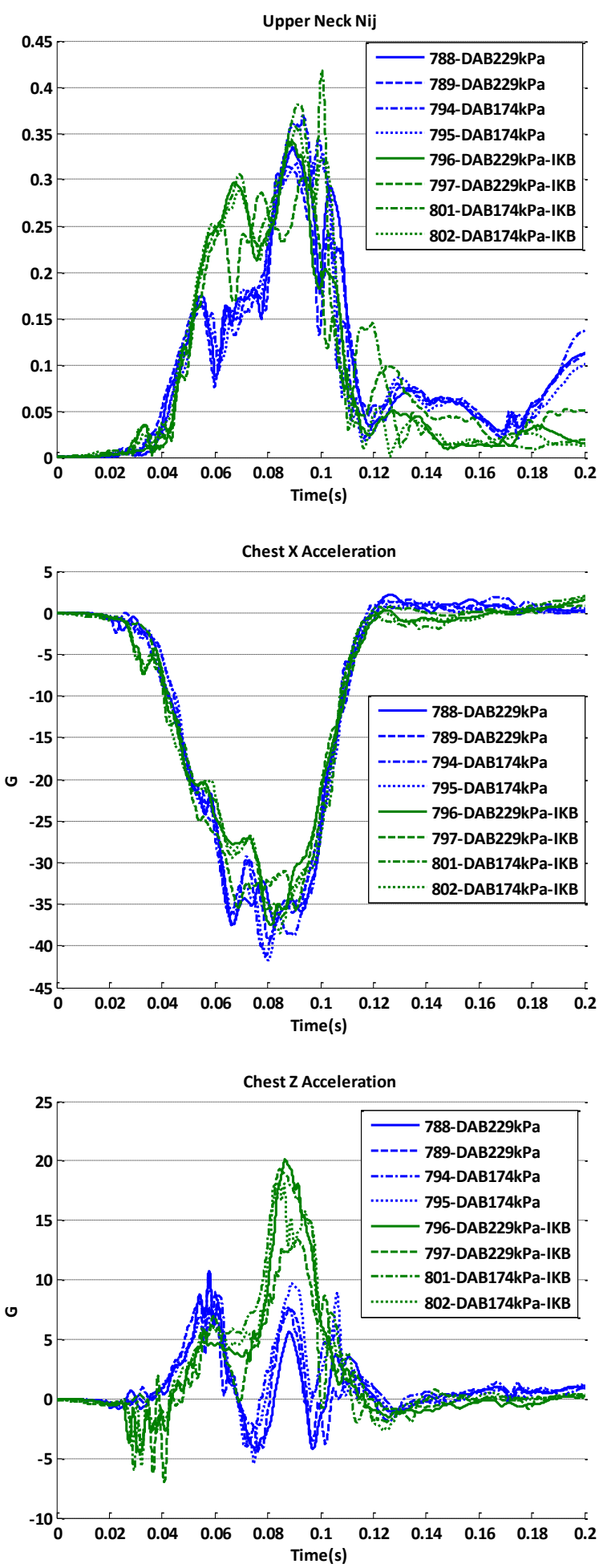
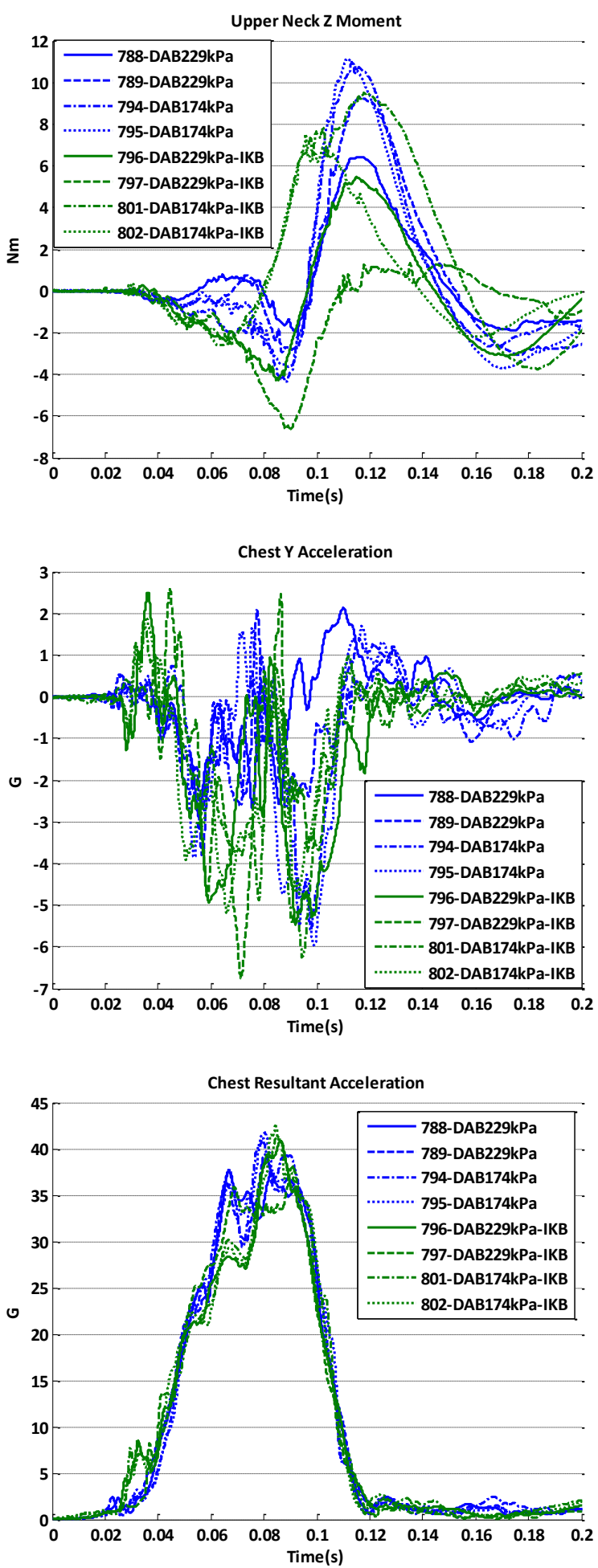

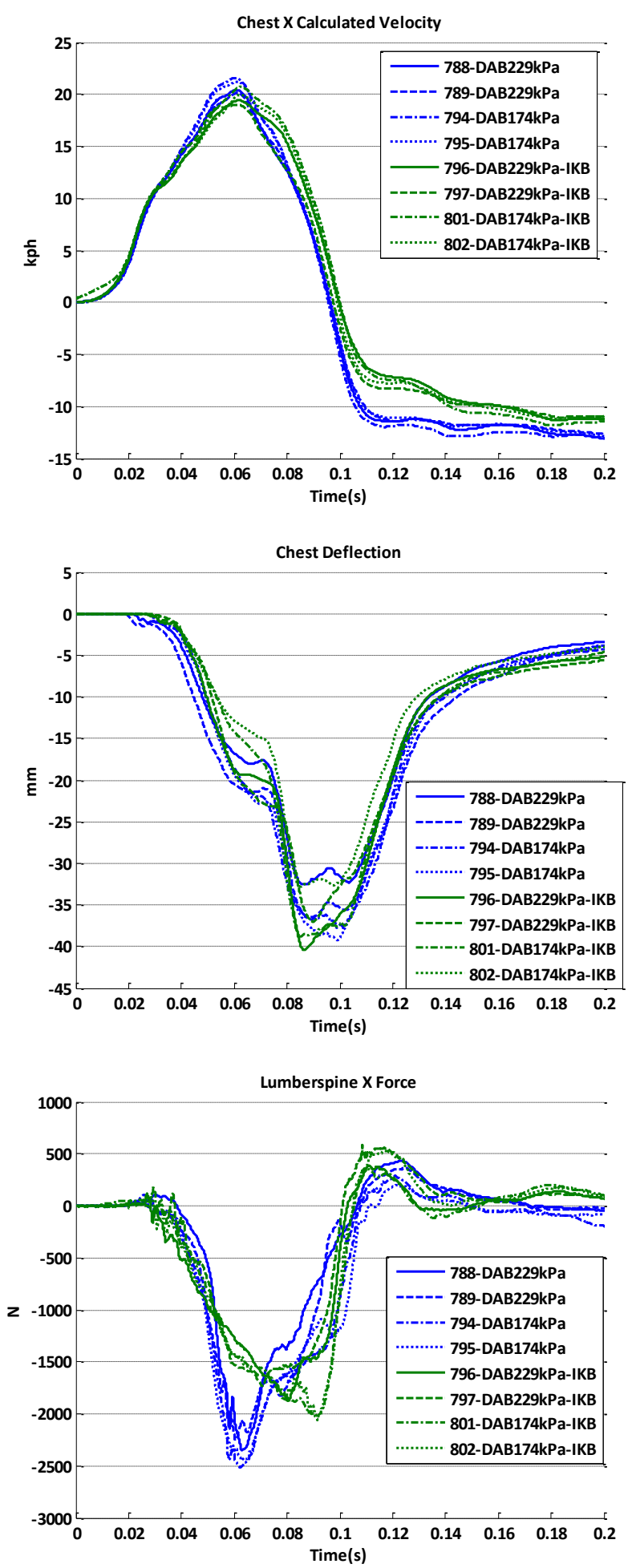
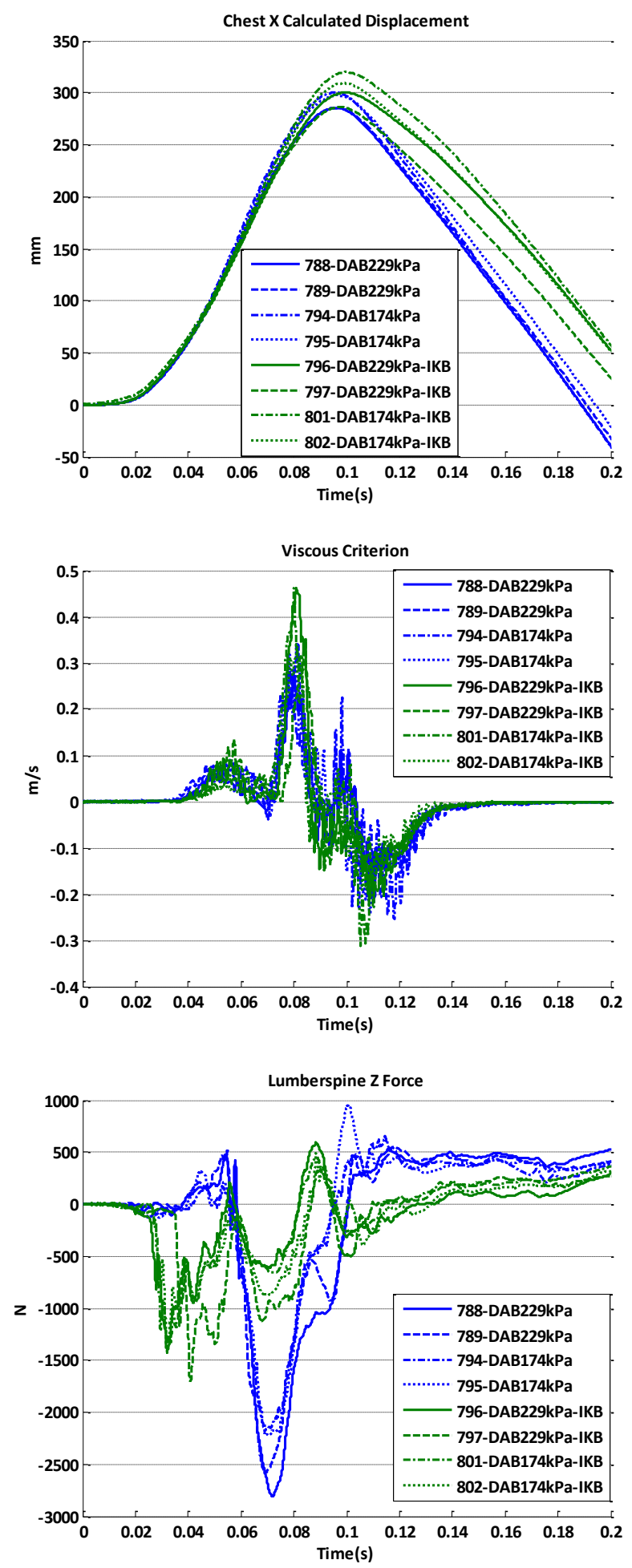

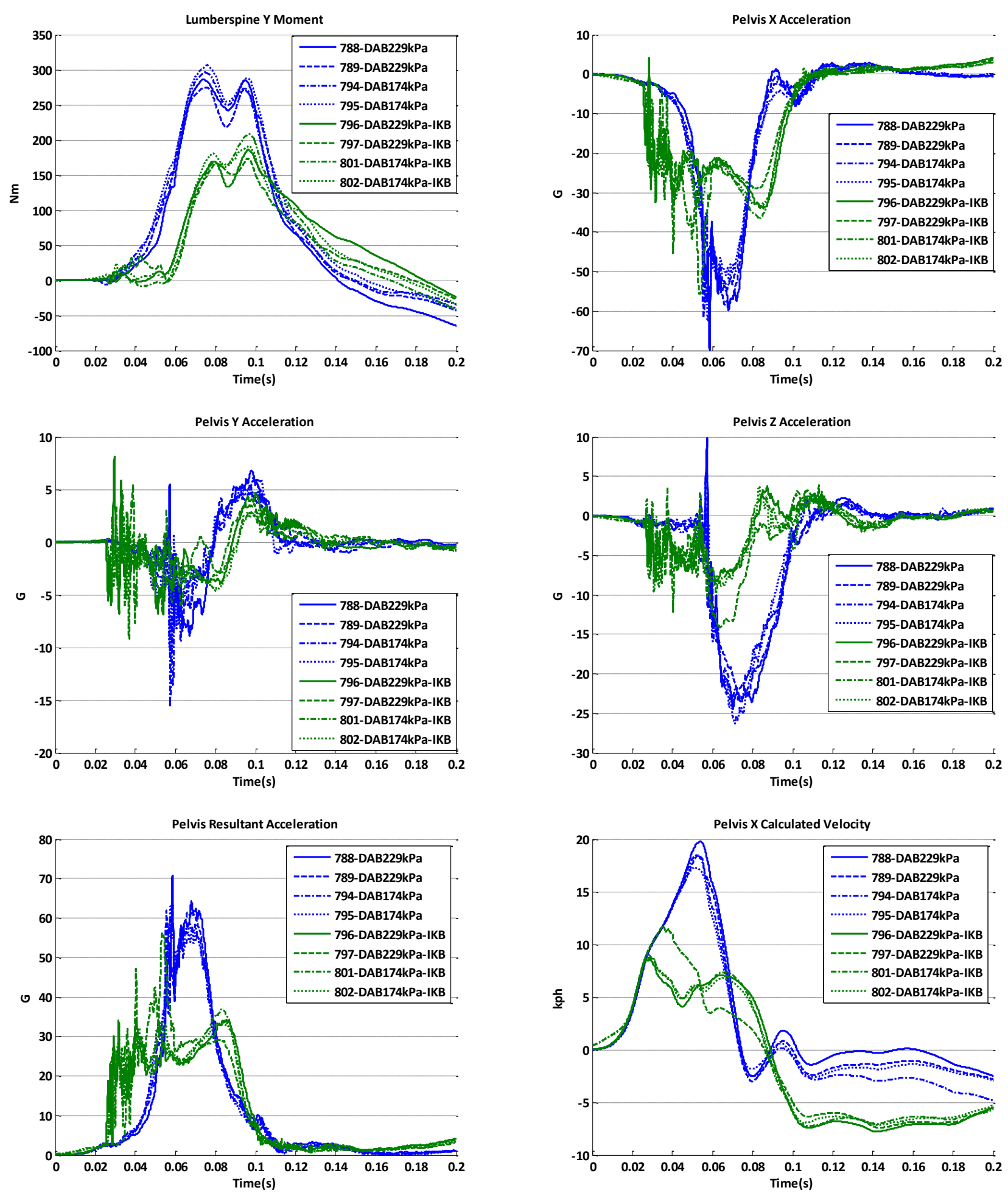

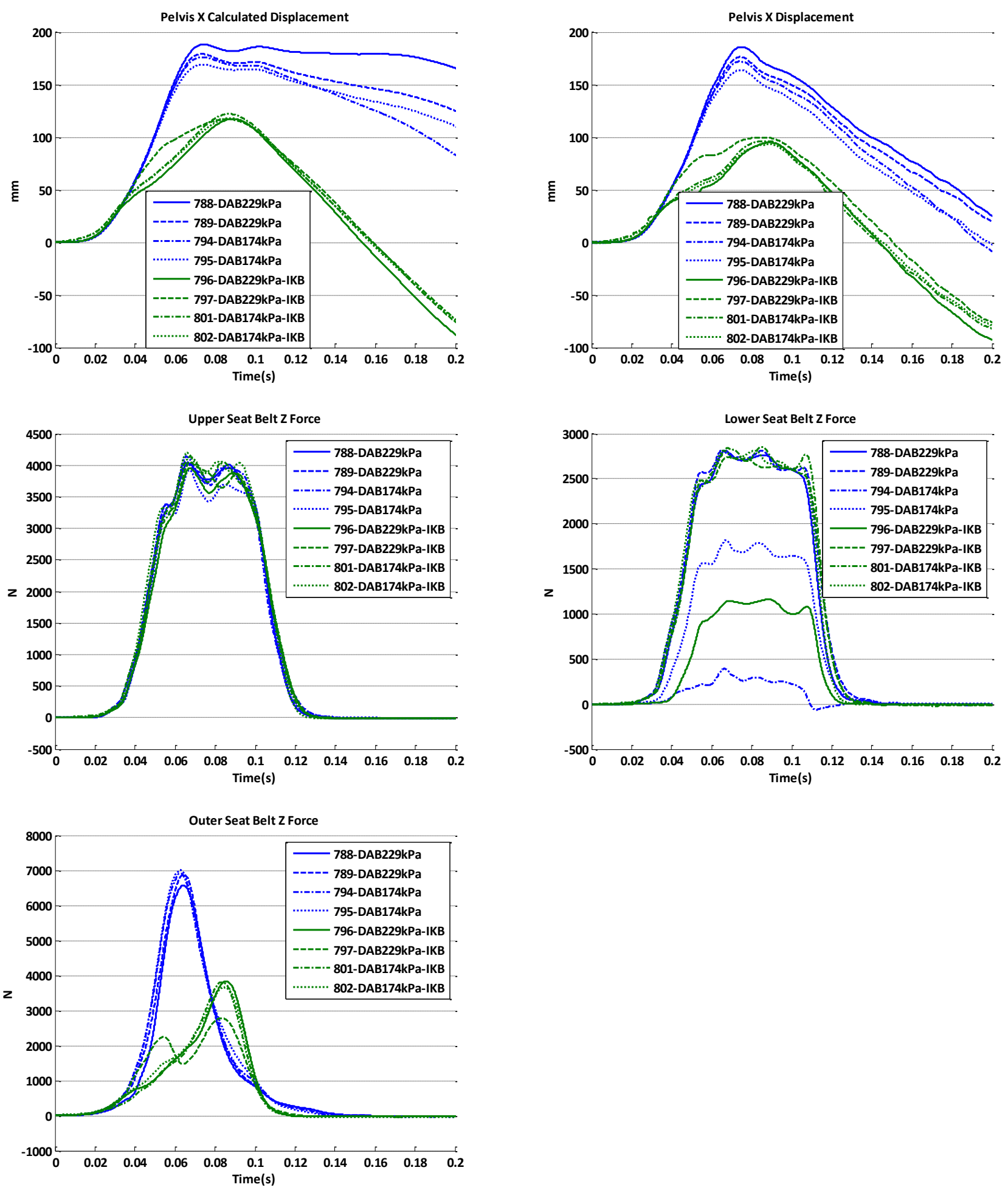
Appendix B. Summary of dummy positioning for all out-of-position tests

\begin{tabular}{|c|c|c|c|c|c|c|c|}
\hline $\begin{array}{l}\text { Measurement } \\
\text { Description }\end{array}$ & $\begin{array}{c}\text { Seatback } \\
\text { angle } \\
\text { (center } \\
\text { line) }\end{array}$ & $\begin{array}{l}\text { Seat angle } \\
\text { ( headrest) }\end{array}$ & $\begin{array}{l}\text { Seat } \\
\text { Height } \\
(\mathrm{mm})\end{array}$ & $\begin{array}{c}\text { Pelvic } \\
\text { angle(Right) }\end{array}$ & $\begin{array}{c}\text { Pelvic } \\
\text { angle(Left) }\end{array}$ & $\begin{array}{c}\text { Femur } \\
\text { angle(Right) }\end{array}$ & $\begin{array}{c}\text { Femur } \\
\text { angle(Left) }\end{array}$ \\
\hline $\begin{array}{l}\text { FMVSS208 } \\
\text { Reference }\end{array}$ & $74^{\circ}$ & $85.7^{\circ}$ & $\begin{array}{l}\text { Mid- } \\
\text { height }\end{array}$ & $21.7^{\circ}$ & $21.7^{\circ}$ & & \\
\hline Notes & & & $\begin{array}{l}\text { seat } \\
\text { bottom to } \\
\text { ground in } \\
\text { vertical } \\
\text { direction }\end{array}$ & & & $\begin{array}{l}\text { H-point to } \\
\text { knee joint } \\
\text { center }\end{array}$ & $\begin{array}{l}\text { H-point to } \\
\text { knee joint } \\
\text { center }\end{array}$ \\
\hline H3-BAS1 & $68.7^{\circ}$ & $85.5^{\circ}$ & 280 & $17.3^{\circ}$ & $17.1^{\circ}$ & $13.0^{\circ}$ & $10.6^{\circ}$ \\
\hline H3-BAS2 & $68.7^{\circ}$ & $85.5^{\circ}$ & 280 & $17.6^{\circ}$ & $17.0^{\circ}$ & $12.1^{\circ}$ & $9.8^{\circ}$ \\
\hline H3-FWD & $68.7^{\circ}$ & $85.5^{\circ}$ & 280 & $19.4^{\circ}$ & $19.0^{\circ}$ & $9.3^{\circ}$ & $8.7^{\circ}$ \\
\hline H3-ADD1 & $68.7^{\circ}$ & $85.5^{\circ}$ & 280 & $20.6^{\circ}$ & $19.9^{\circ}$ & $9.2^{\circ}$ & $9.0^{\circ}$ \\
\hline H3-ADD2 & $68.7^{\circ}$ & $85.5^{\circ}$ & 280 & $19.4^{\circ}$ & $19.2^{\circ}$ & $8.8^{\circ}$ & $7.5^{\circ}$ \\
\hline THOR-BAS1 & $68.5^{\circ}$ & $85.5^{\circ}$ & 280 & $16.1^{\circ}$ & $15.6^{\circ}$ & $12.0^{\circ}$ & $11.3^{\circ}$ \\
\hline THOR-BAS2 & $68.5^{\circ}$ & $85.5^{\circ}$ & 280 & $14.9^{\circ}$ & $14.3^{\circ}$ & $12.9^{\circ}$ & $9.6^{\circ}$ \\
\hline THOR-FWD & $68.5^{\circ}$ & $85.5^{\circ}$ & 280 & $17.0^{\circ}$ & $16.4^{\circ}$ & $12.4^{\circ}$ & $8.5^{\circ}$ \\
\hline THOR-ADD & $68.5^{\circ}$ & $85.5^{\circ}$ & 280 & $14.9^{\circ}$ & $14.1^{\circ}$ & $16.3^{\circ}$ & $4.5^{\circ}$ \\
\hline THOR-ADD2 & $68.5^{\circ}$ & $85.5^{\circ}$ & 280 & $21.2^{\circ}$ & $20.8^{\circ}$ & $11.8^{\circ}$ & $12.3^{\circ}$ \\
\hline THOR-ADD1 & $68.5^{\circ}$ & $85.5^{\circ}$ & 280 & $19.1^{\circ}$ & $18.3^{\circ}$ & $13.6^{\circ}$ & $12.3^{\circ}$ \\
\hline $\begin{array}{l}\text { Measurement } \\
\text { Description }\end{array}$ & $\begin{array}{l}\text { Tibia } \\
\text { angle } \\
\text { (Right) }\end{array}$ & $\begin{array}{c}\text { Tibia angle } \\
\text { (Left) }\end{array}$ & $\begin{array}{l}\text { Knee to } \\
\text { Knee } \\
\text { (mm) }\end{array}$ & $\begin{array}{l}\text { Left knee to } \\
\text { dash(mm) }\end{array}$ & $\begin{array}{l}\text { Tibia to KAB } \\
\text { module }(\mathrm{mm})\end{array}$ & $\begin{array}{c}\text { Right knee } \\
\text { to } \\
\text { dash }(\mathrm{mm})\end{array}$ & \\
\hline $\begin{array}{l}\text { FMVSS } 208 \\
\text { Reference }\end{array}$ & $51.6^{\circ}$ & $51.6^{\circ}$ & 252 & 82 & & 85 & \\
\hline Notes & & $\begin{array}{c}\text { Knee joint } \\
\text { to ankle Y- } \\
\text { rotation } \\
\text { bolt joint } \\
\text { center }\end{array}$ & $\begin{array}{l}\text { Distance } \\
\text { between } \\
\text { knee } \\
\text { centerline }\end{array}$ & $\begin{array}{l}\text { Lateral } \\
\text { knee joint } \\
\text { center to } \\
\text { closest IP }\end{array}$ & $\begin{array}{l}\text { Anterior } \\
\text { tibia surface } \\
\text { to center of } \\
\text { KAB module }\end{array}$ & $\begin{array}{l}\text { Lateral } \\
\text { knee joint } \\
\text { center to } \\
\text { closest IP }\end{array}$ & $\begin{array}{l}\text { Anterior } \\
\text { tibia surface } \\
\text { to center of } \\
\text { KAB module }\end{array}$ \\
\hline H3-BAS1 & $70.3^{\circ}$ & $60.1^{\circ}$ & 251 & 81 & NA & 66 & NA \\
\hline H3-BAS2 & NA & NA & 249 & 85 & NA & 67 & NA \\
\hline H3-FWD & NA & NA & 252 & 67 & 2.7 & 68 & 7.3 \\
\hline H3-ADD1 & NA & NA & 180 & 73 & 1.9 & 63 & 12.5 \\
\hline H3-ADD2 & NA & NA & 158 & 77 & 0.5 & 65 & 18 \\
\hline THOR-BAS1 & $77.1^{\circ}$ & $58.9^{\circ}$ & 248 & 82 & 43.0 & 76 & 51 \\
\hline THOR-BAS2 & $67.6^{\circ}$ & $57.4^{\circ}$ & 250 & 82 & 47.0 & 73 & 54 \\
\hline THOR-FWD & $50.3^{\circ}$ & $49.4^{\circ}$ & 249 & 72 & 8.0 & 70 & 11 \\
\hline THOR-ADD & $59.0^{\circ}$ & $42.0^{\circ}$ & 180 & 99 & 25.0 & 65 & 32 \\
\hline THOR-ADD2 & $70.0^{\circ}$ & $67.2^{\circ}$ & 158 & 68 & 46.0 & 67 & 57 \\
\hline THOR-ADD1 & $72.1^{\circ}$ & $62.1^{\circ}$ & 180 & 71 & 29.0 & 65 & 55 \\
\hline
\end{tabular}


Additional measurements: seat bottom to ground in vertical direction: $280 \mathrm{~mm}$; seat front to IP in horizontal direction: $305 \mathrm{~mm}$; seat width $432 \mathrm{~mm}$;

seat height top to ground: $782 \mathrm{~mm}$ (vertical); H-point to ground :365 mm (vertical) ; seat bottom inclined angle: $8.2^{\circ}$.

Appendix C. Airbag mass flow rate and tank pressure during deployment for out-of-position tests

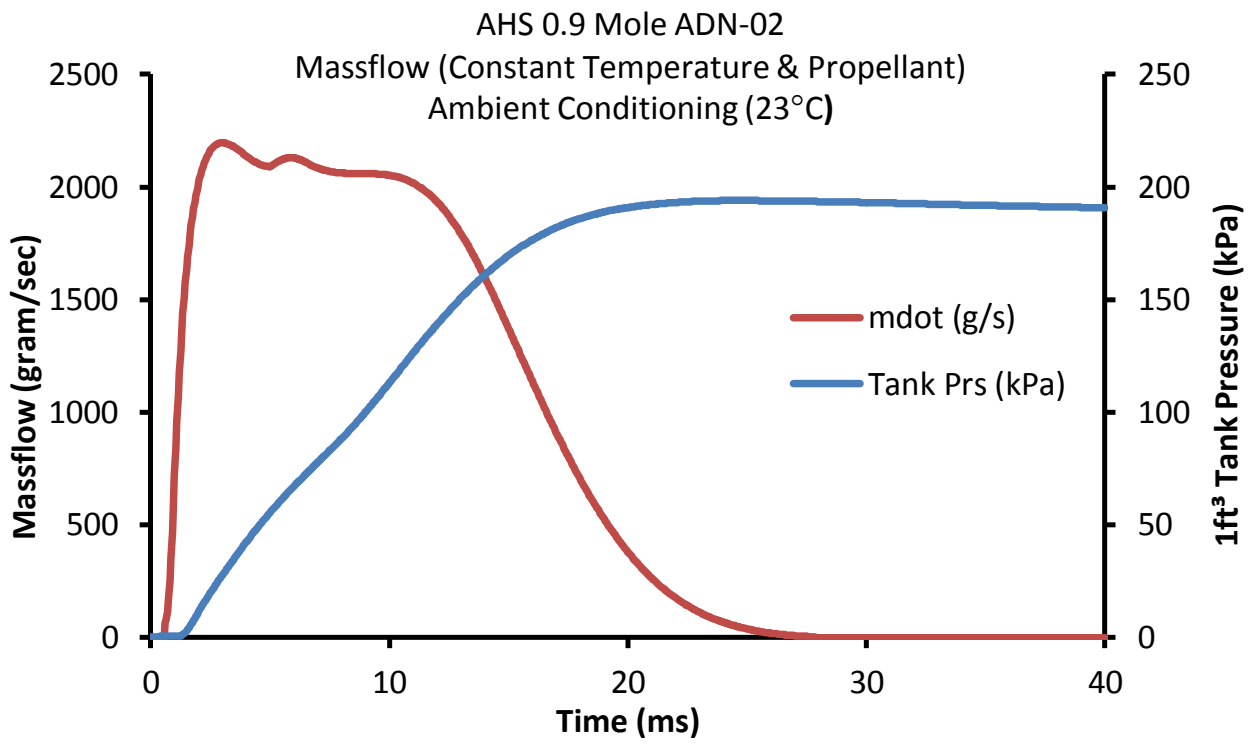

Appendix D. Injury assessment reference values from IIHS

\begin{tabular}{ccccc}
\hline 5th Female ATD & \multicolumn{4}{c}{ Rating Boundary Values } \\
\hline tibia index & Good & Acceptable & Marginal & Poor \\
tibia axial force $(\mathrm{N})$ & $<0.8$ & $0.8-1.0$ & $1.0-1.2$ & $>1.2$ \\
foot acceleration $(\mathrm{X}, \mathrm{Z})(\mathrm{g})$ & $<2600$ & $2600-3900$ & $3900-5100$ & $>5100$ \\
femur force $(\mathrm{N})$ & $<150$ & $150-200$ & $200-260$ & $>260$ \\
\hline
\end{tabular}




\section{Appendix E. Tests data for out-of-position knee airbag tests}
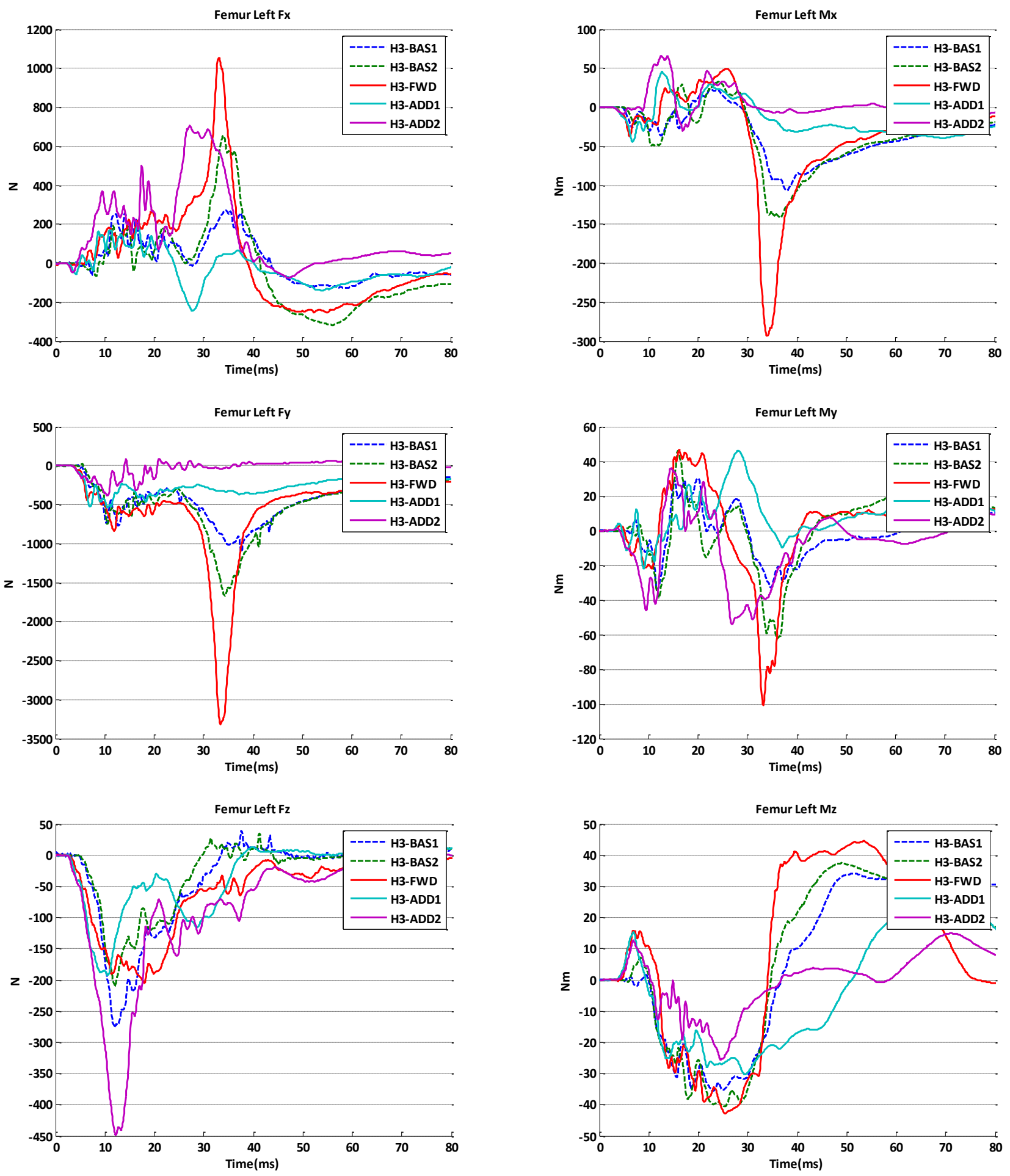

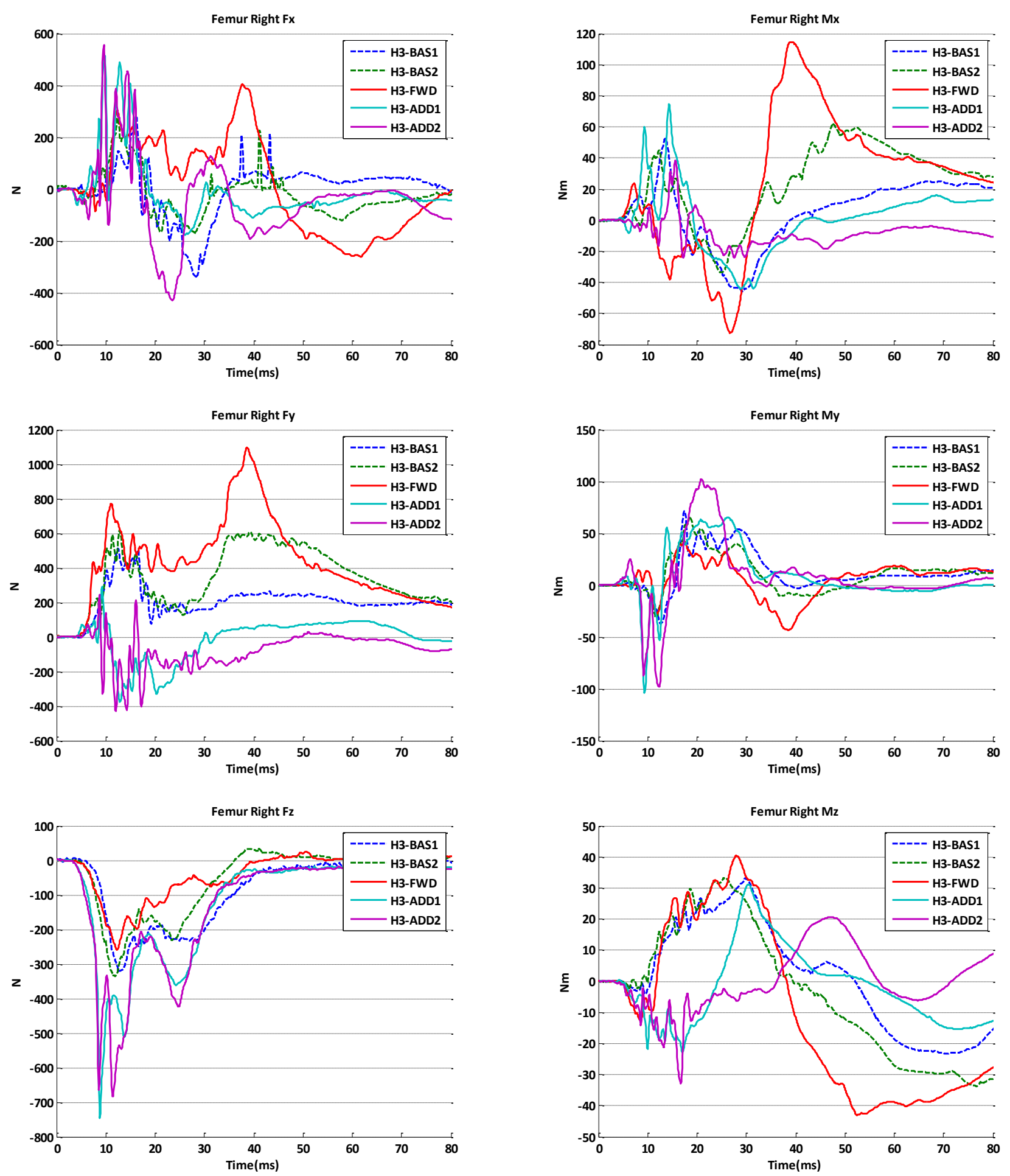

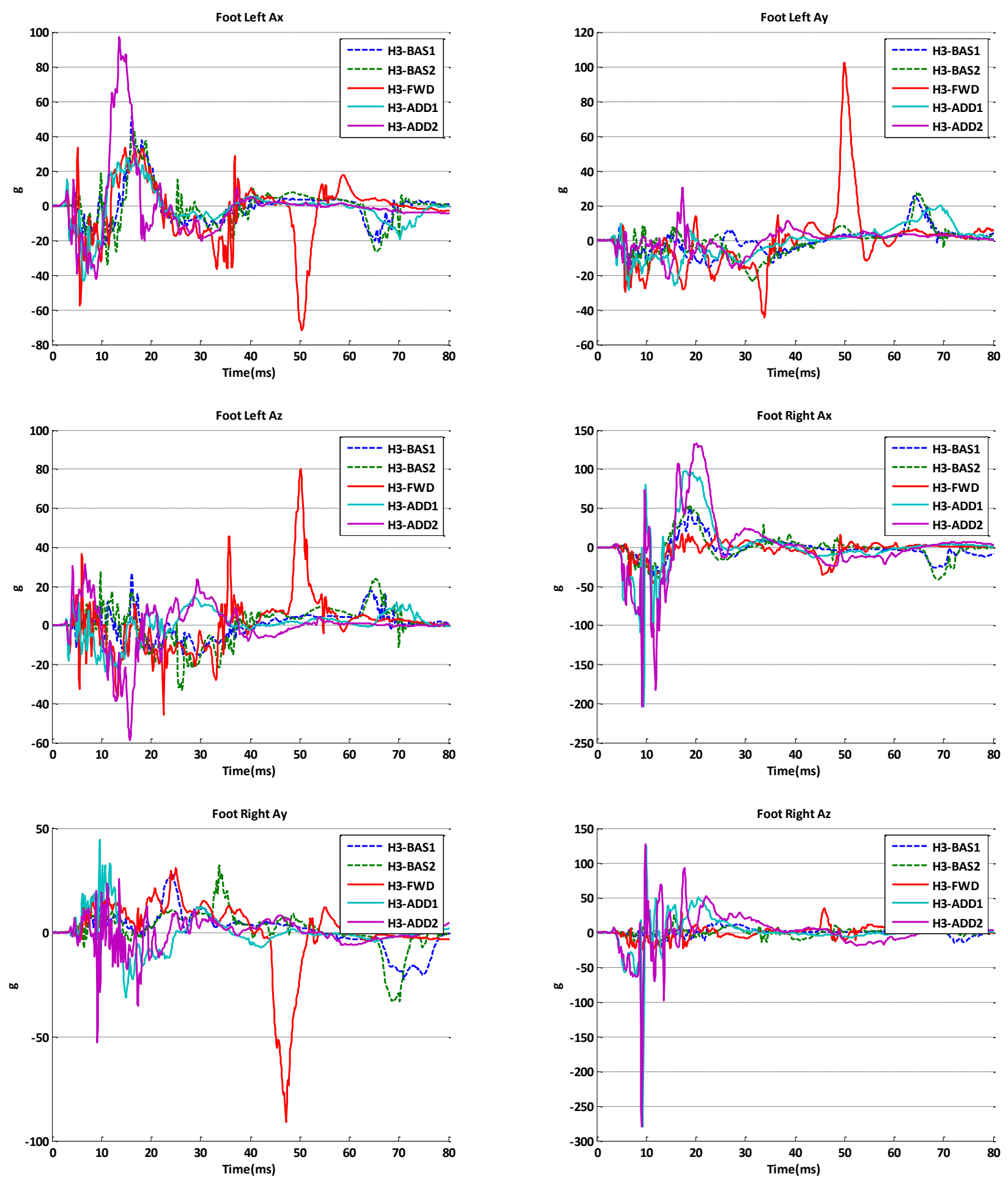

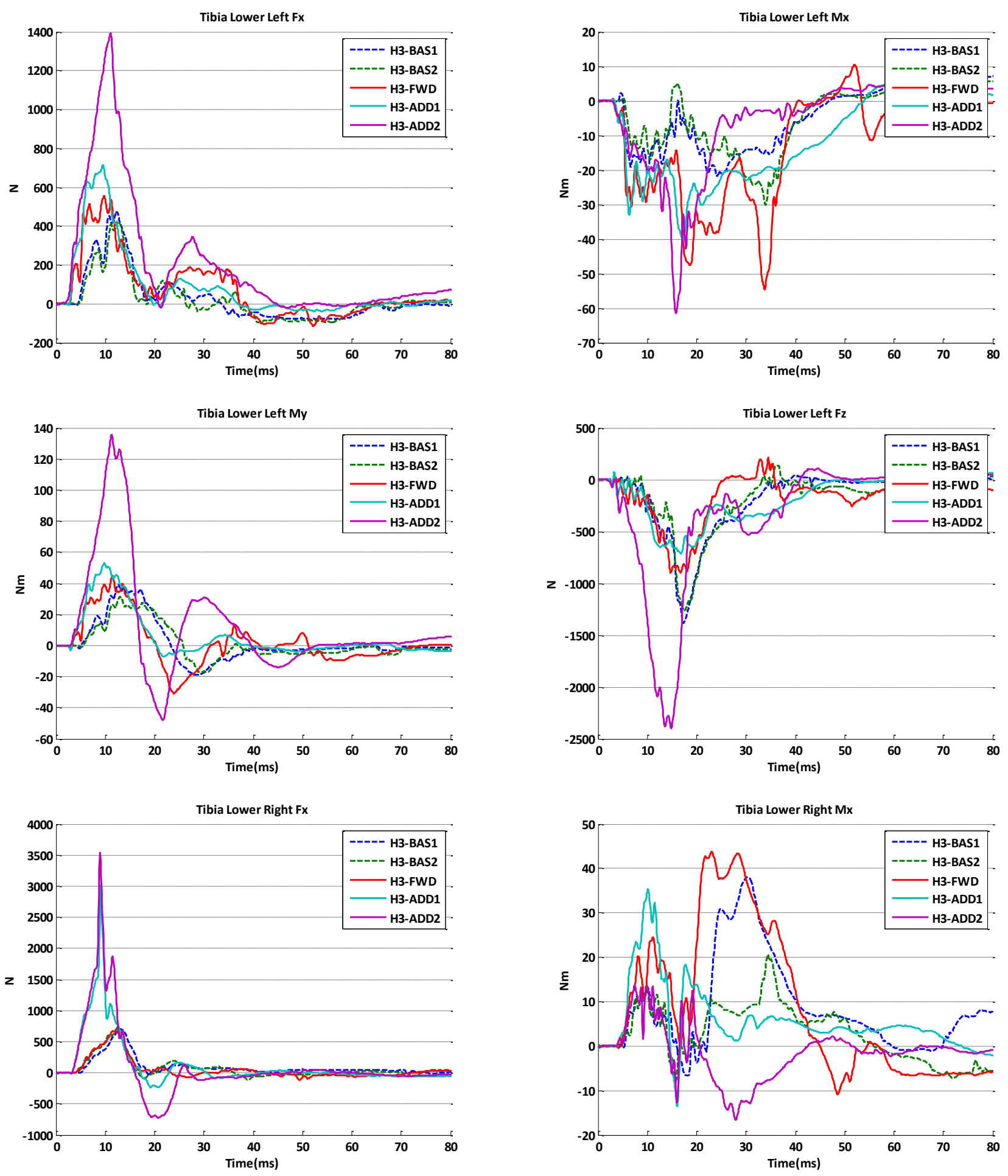

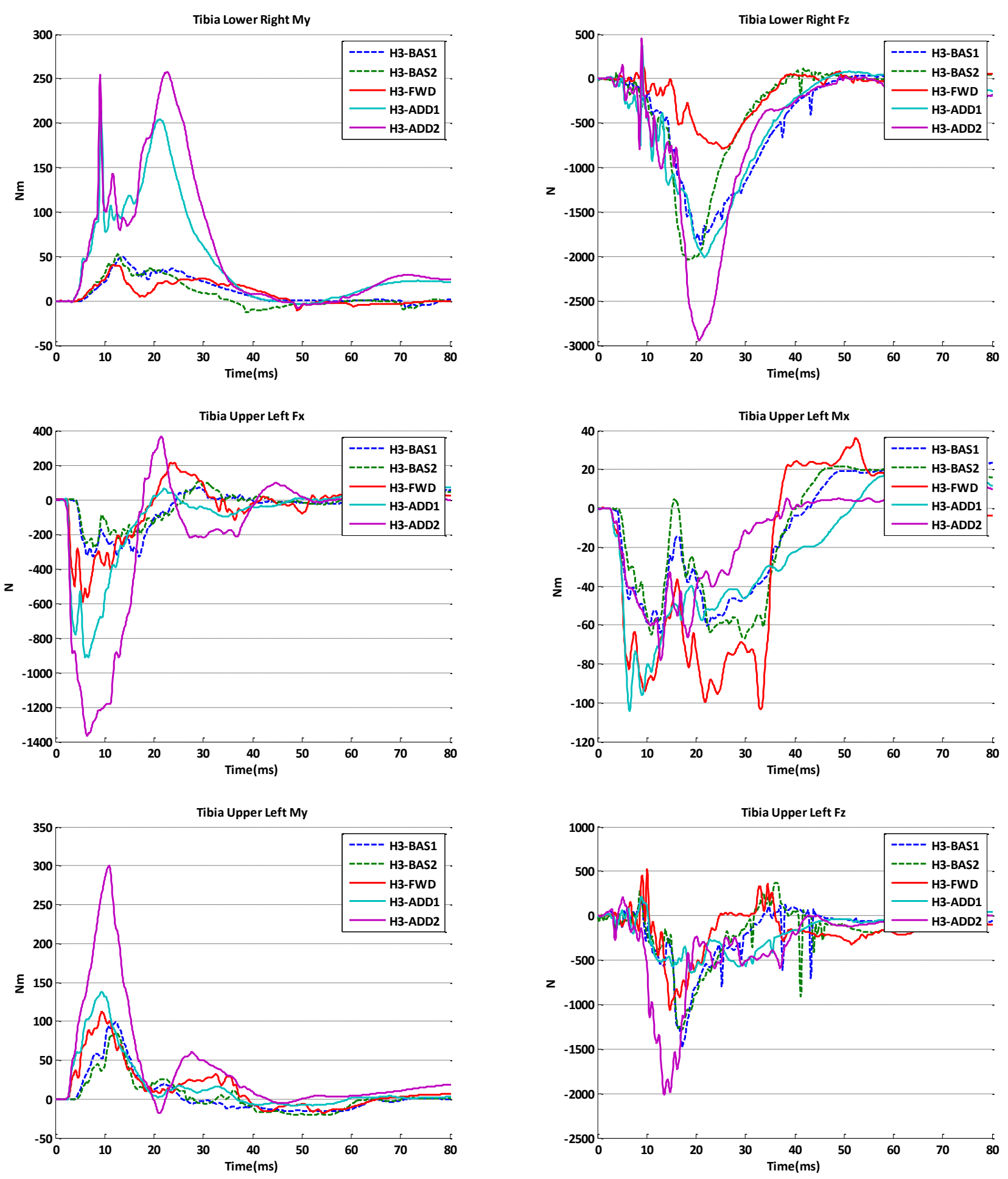

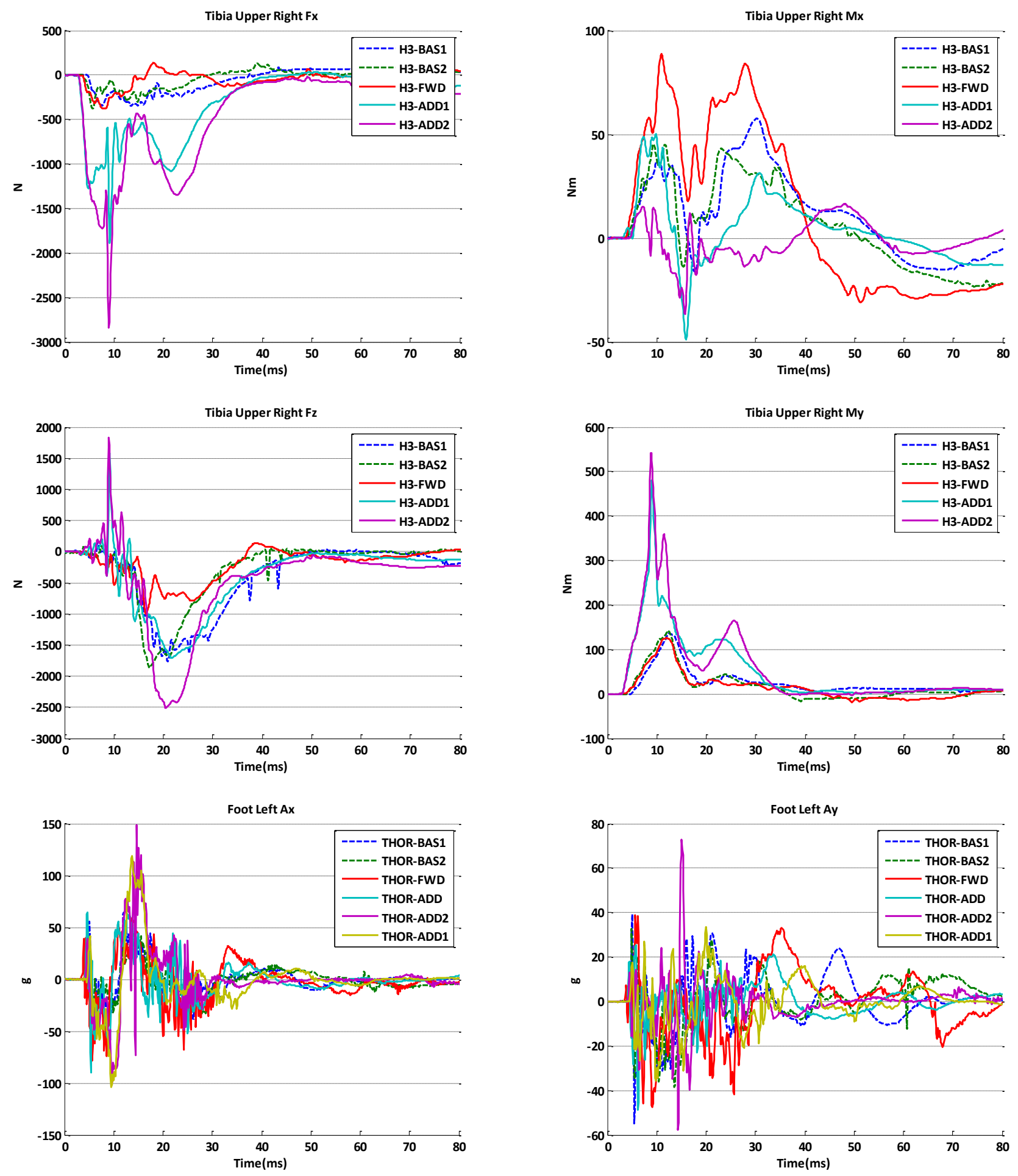

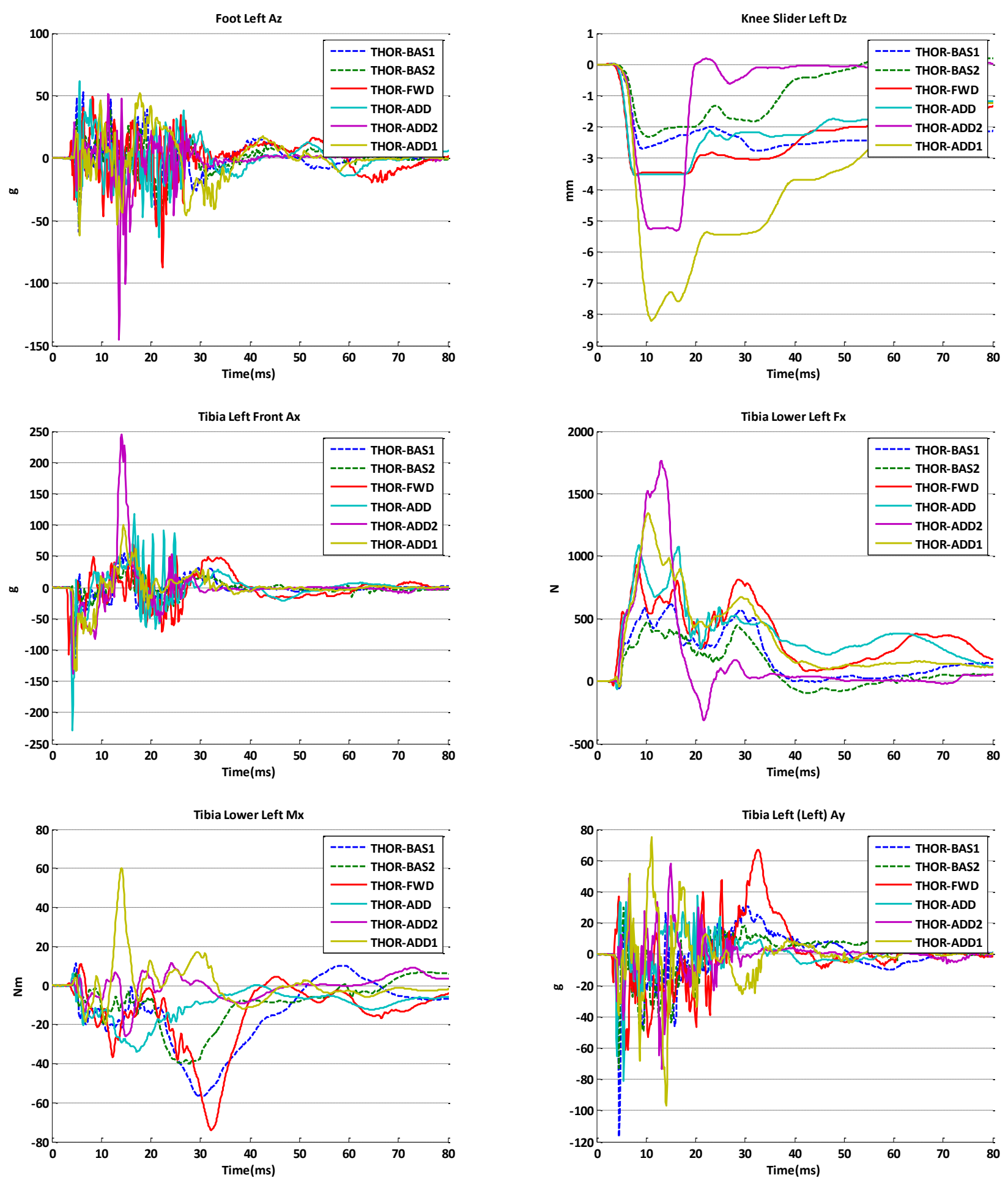

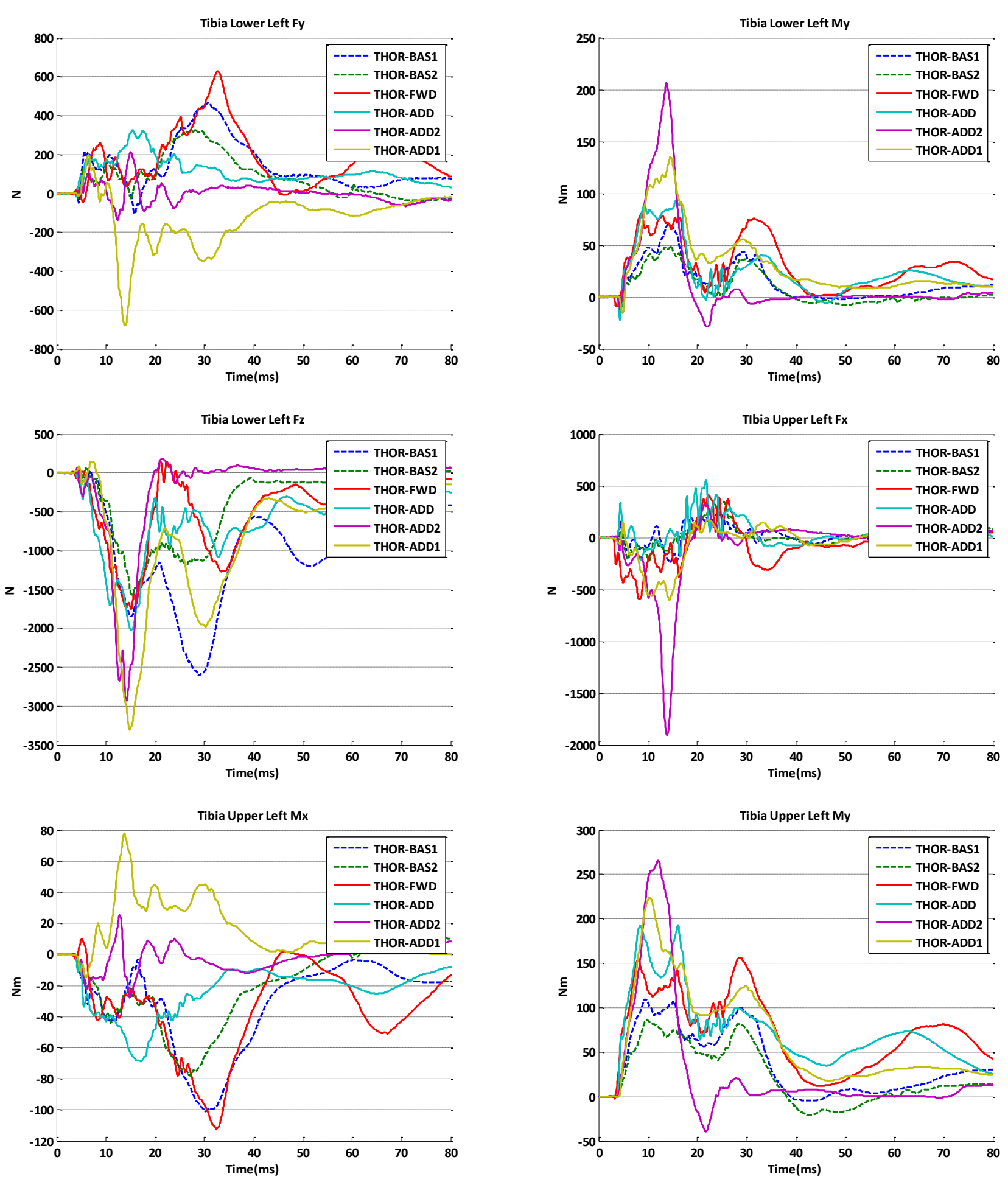

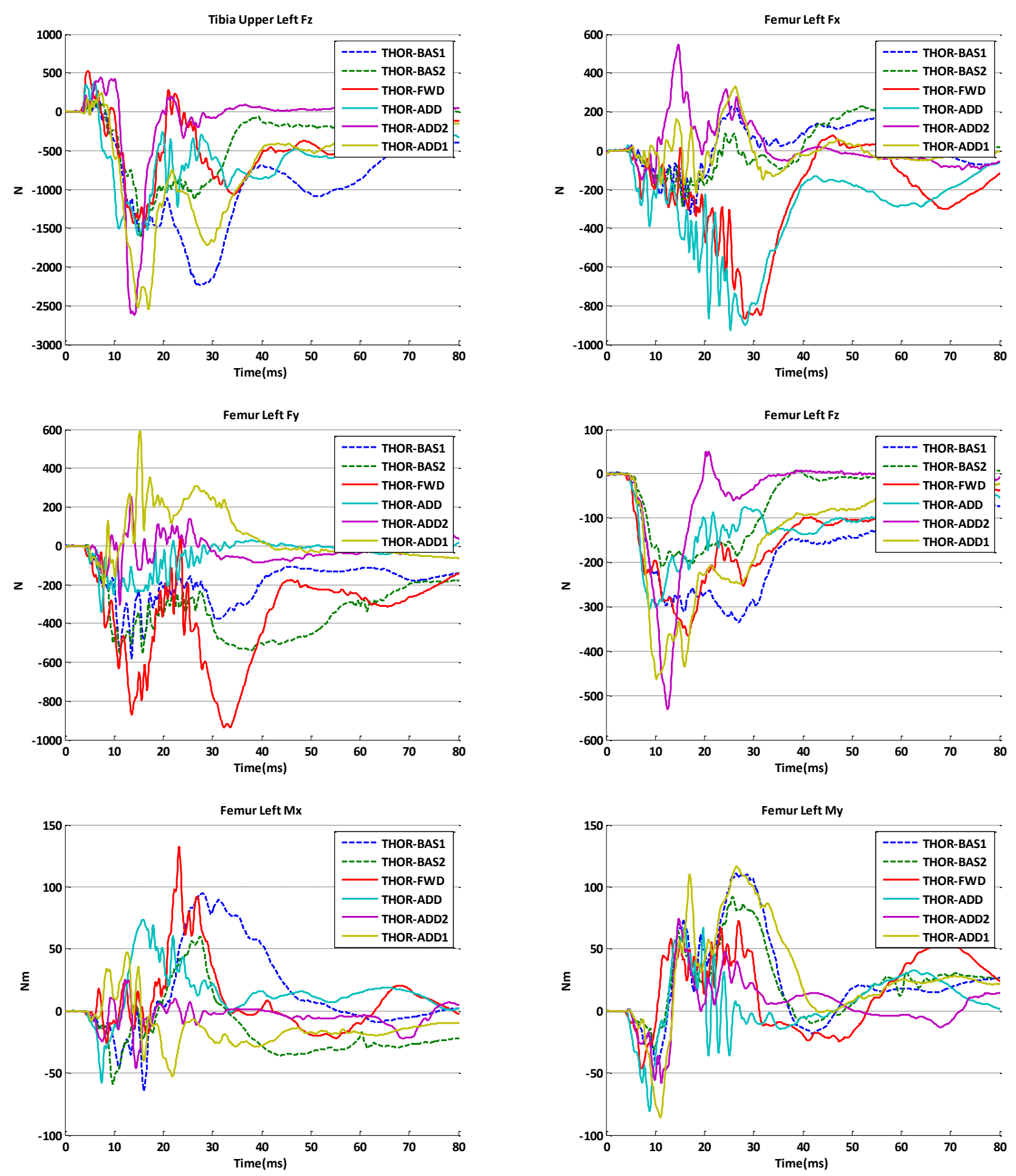

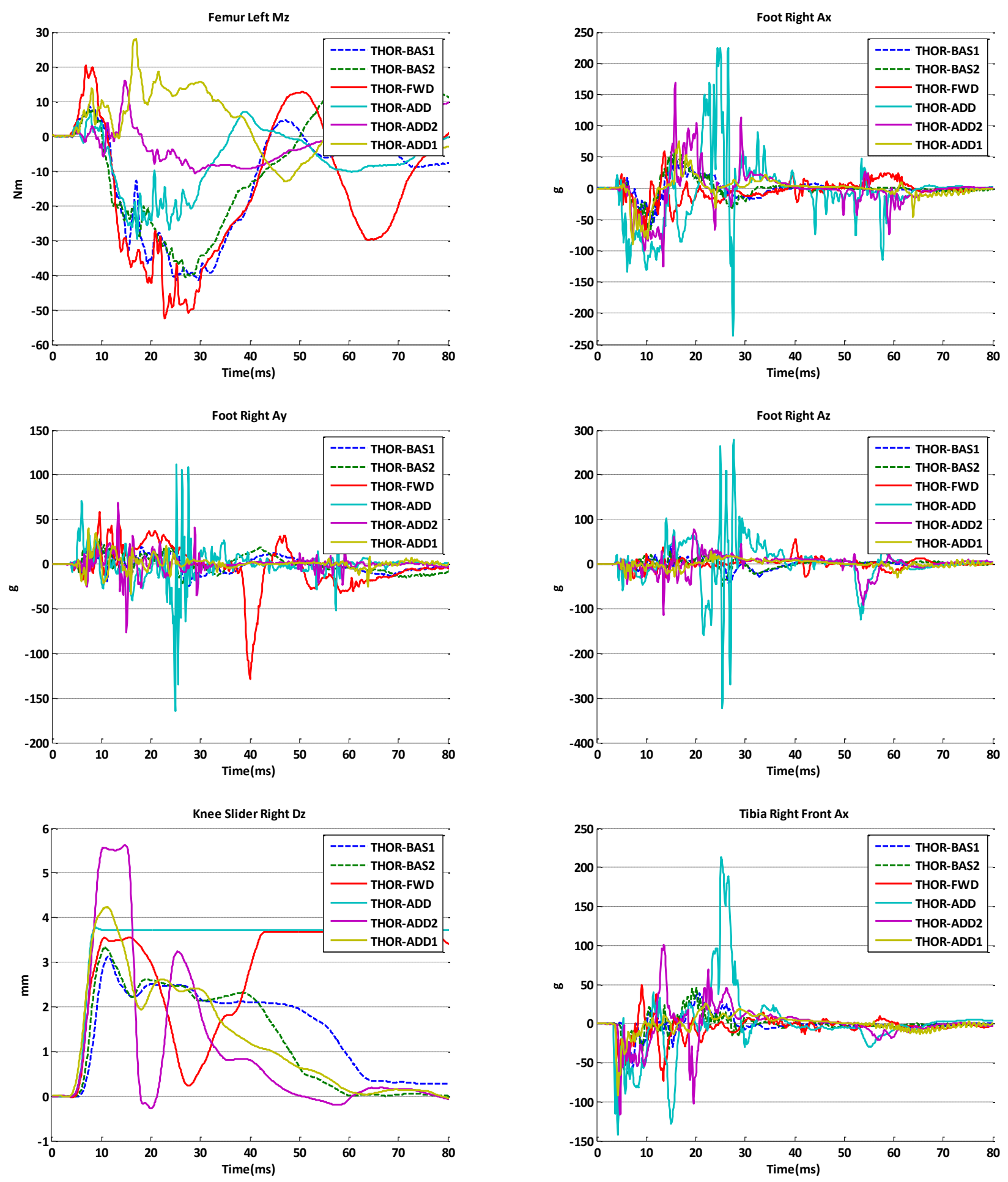

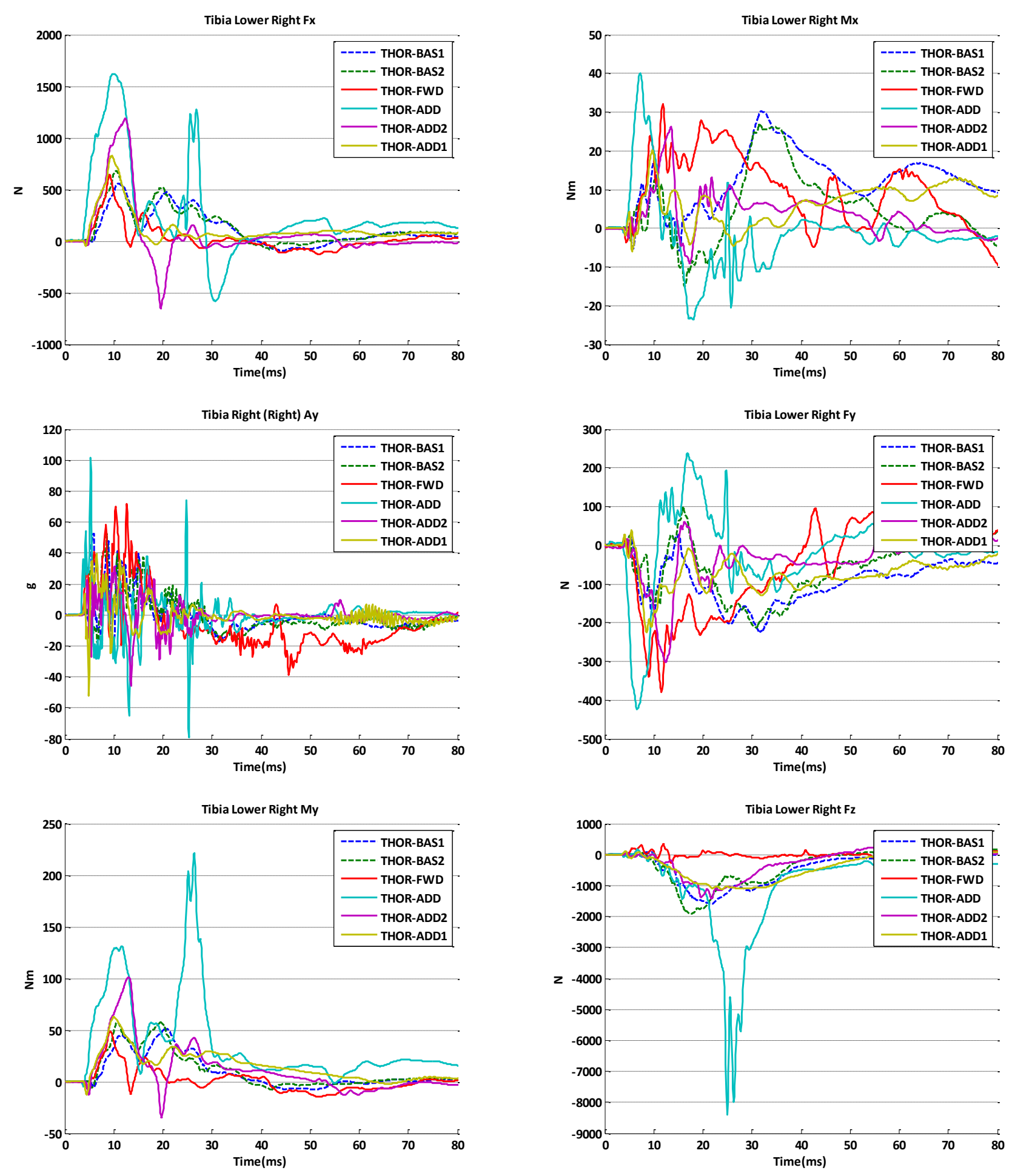

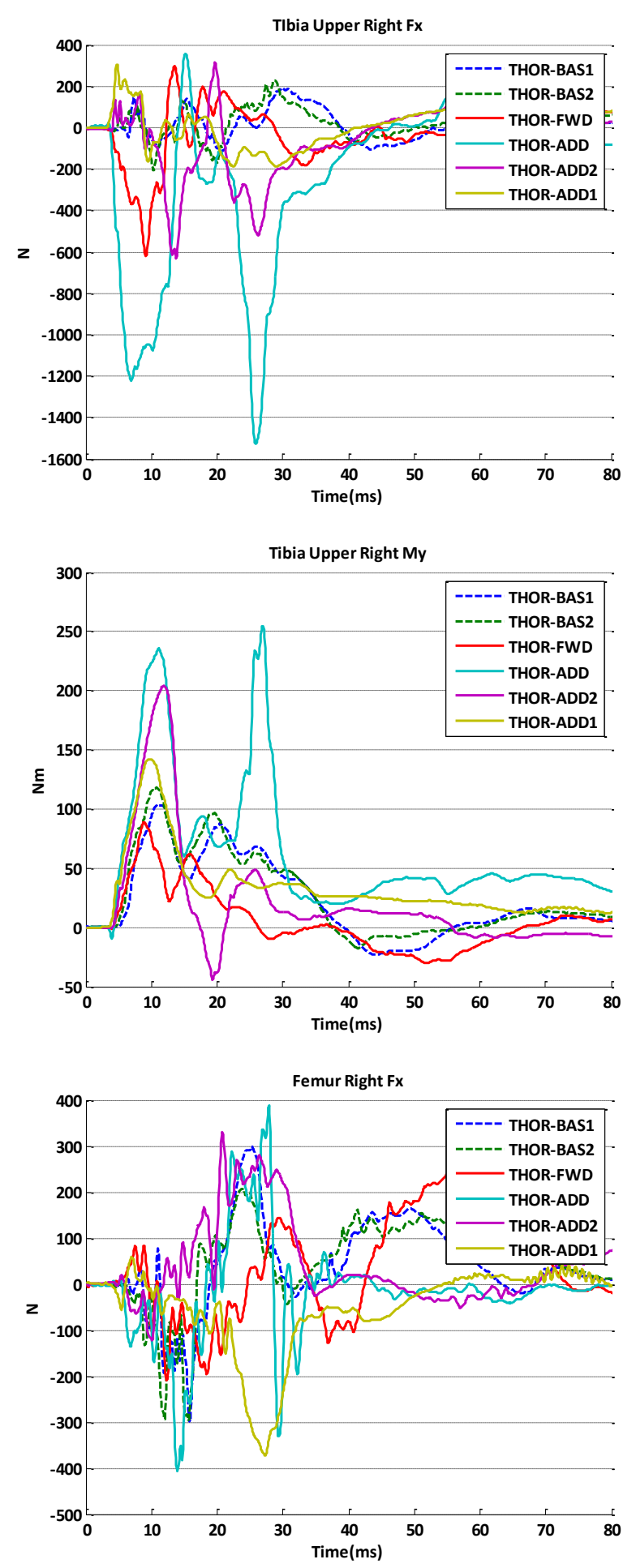
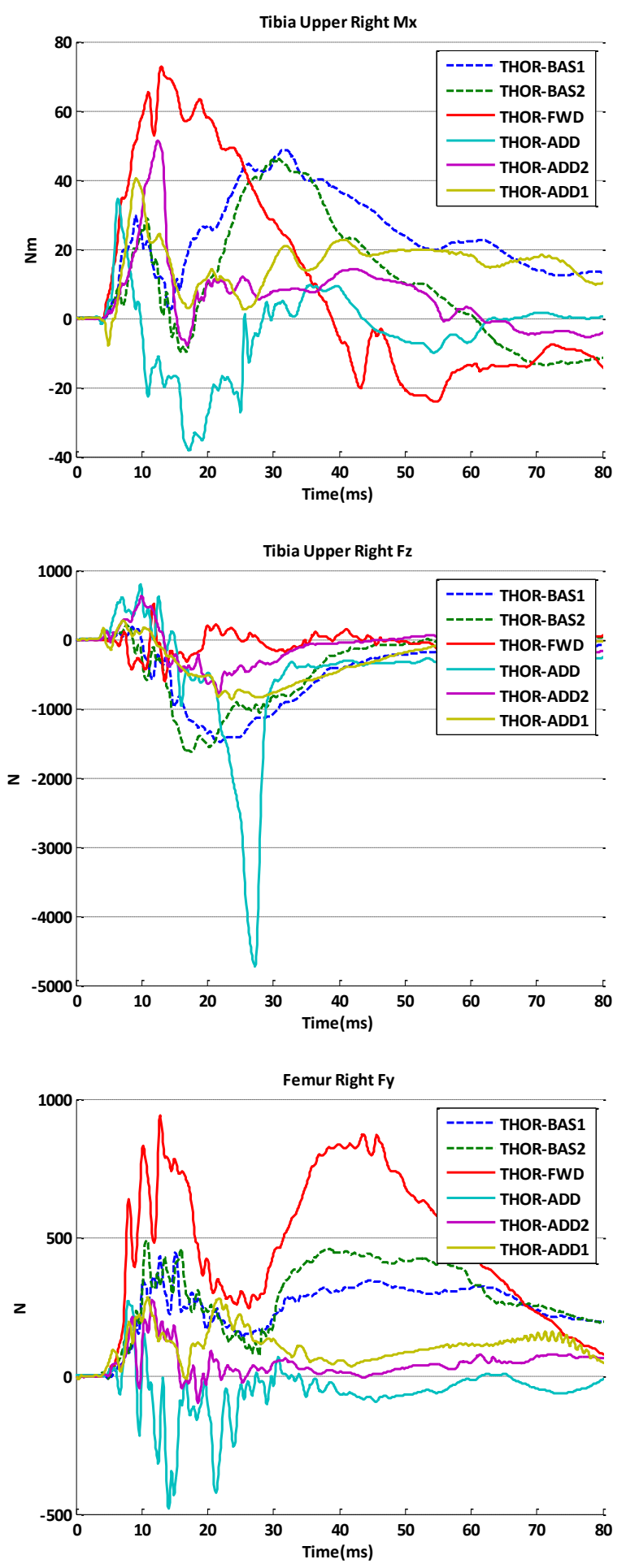

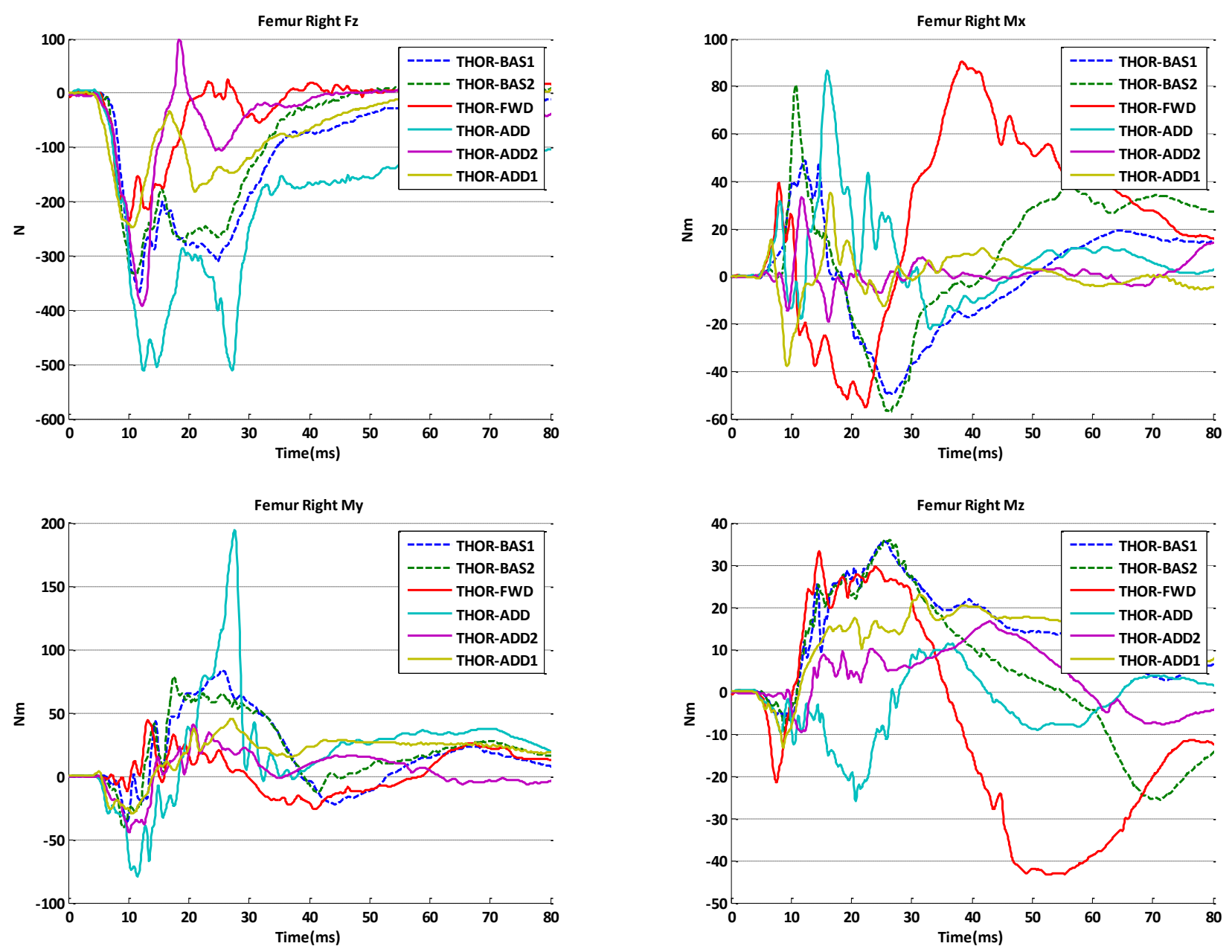
Appendix F. Data collection of vehicle fleets equipped with knee airbag in the U.S market

(SOP Year: 2001 -2014; Sources: OEMs, NHTSA)

\begin{tabular}{|c|c|c|c|c|c|}
\hline SOP & Brand & Platform & Model & $\begin{array}{c}\text { Driver } \\
\text { KAB }\end{array}$ & $\begin{array}{c}\text { Passenger } \\
\text { КАВ }\end{array}$ \\
\hline 2001 & BMW & 7-SERIES & E65 & $\mathrm{Y}$ & $\mathrm{Y}$ \\
\hline 2001 & MERCEDES & SL & R230 & Y & N/A \\
\hline 2001 & LAND ROVER & Range Rover & L322 & Y & N/A \\
\hline 2002 & BMW & Z4 & Z4 & Y & $\mathrm{Y}$ \\
\hline 2002 & MERCEDES & E-Class Sedan & W211 & Y & N/A \\
\hline 2003 & BMW & 6-SERIES & E63 & Y & $\mathrm{Y}$ \\
\hline 2003 & BMW & 6-SERIES & E64 & Y & Y \\
\hline 2003 & ROLLS ROYCE & Rolls Royce & RR01 & Y & Y \\
\hline 2003 & MERCEDES & E-Class Wagon & S211 & Y & N/A \\
\hline 2003 & LEXUS & RX, RX330 & $480 N$ & Y & $\mathrm{N}$ \\
\hline 2003 & AUDI & A8 & AU631 & Y & $\mathrm{Y}$ \\
\hline 2003 & BENTLEY & CONTINENTAL GT & BY 614 & $\mathrm{~N}$ & $\mathrm{Y}$ \\
\hline 2004 & CHRYSLER & Pacifica/High Line & CS & Y & $\mathrm{N}$ \\
\hline 2004 & CHRYSLER & Grand Caravan & RG & $\mathrm{N}$ & $\mathrm{Y}$ \\
\hline 2004 & MERCEDES & E-Class Coupe & C219 & Y & N/A \\
\hline 2004 & MERCEDES & Maybach & Maybach & Y & $\mathrm{Y}$ \\
\hline 2004 & MERCEDES & S Class SLSS & S Class & Y & $\mathrm{N}$ \\
\hline 2004 & JAGUAR & X-type (Estate Wagon) & $x 400$ & Y & $\mathrm{N}$ \\
\hline 2004 & CITROEN & New 406 & & Y & $\mathrm{N}$ \\
\hline 2004 & CITROEN & $\mathrm{C} 6$ & $x 6$ & Y & $\mathrm{N}$ \\
\hline 2004 & PEUGEOT & New XM & & Y & $\mathrm{N}$ \\
\hline 2004 & LEXUS & LS 430 & & Y & $\mathrm{Y}$ \\
\hline 2004 & LEXUS & $\mathrm{GS}(300,450,500)$ & $620 N$ & Y & $\mathrm{Y}$ \\
\hline 2005 & CHRYSLER & PT Cruiser & PT & Y & $\mathrm{N}$ \\
\hline 2005 & CHRYSLER & Caliber & PM & Y & $\mathrm{N}$ \\
\hline 2005 & LEXUS & IS (350) & 030L & Y & $\mathrm{Y}$ \\
\hline 2005 & LEXUS & $\mathrm{SC}$ & 317L & Y & N/A \\
\hline 2005 & TOYOTA & Avalon & $770 N$ & Y & $\mathrm{N}$ \\
\hline 2006 & BMW & Z4 COUPE & E86 & Y & $\mathrm{Y}$ \\
\hline 2006 & CHRYSLER & Compass/Patriot & MK49/74 & Y & $\mathrm{N}$ \\
\hline 2006 & KIA & SORENTO F/L & $B L F / L$ & Y & $\mathrm{N}$ \\
\hline 2006 & KIA & New Luxury SUV (HM) & Veracruz & Y & $\mathrm{Y}$ \\
\hline 2006 & PEUGEOT & 407 & D22 & Y & $\mathrm{N}$ \\
\hline 2006 & PEUGEOT & 407 & D23 & $Y$ & $\mathrm{~N}$ \\
\hline 2006 & PEUGEOT & 407 & D25 & Y & $\mathrm{N}$ \\
\hline 2006 & LEXUS & ES & 041L & $Y$ & $\mathrm{~N} / \mathrm{A}$ \\
\hline
\end{tabular}




\begin{tabular}{|c|c|c|c|c|c|}
\hline 2006 & LEXUS & LS (460) & $250 \mathrm{~L}$ & $\mathrm{Y}$ & $\mathrm{Y}$ \\
\hline 2006 & TOYOTA & Camry & 044L & $\mathrm{Y}$ & $\mathrm{N}$ \\
\hline 2006 & TOYOTA & RYU & $155 \mathrm{~L}$ & $\mathrm{Y}$ & $\mathrm{N} / \mathrm{A}$ \\
\hline 2006 & AUDI & TT (Coupe/Roadster) & AU324/325 & $\mathrm{Y}$ & Y \\
\hline 2006 & BENTLEY & CONTINENTAL GTC & BY 615 & $\mathrm{Y}$ & Y \\
\hline 2007 & ROLLS ROYCE & ROLLS ROYCE & RR02 & $\mathrm{Y}$ & $\mathrm{Y}$ \\
\hline 2007 & CHRYSLER & Caravan Minivan & RS & $Y$ & $\mathrm{~N}$ \\
\hline 2007 & CHRYSLER & Town and Country Minivan & RS & $\mathrm{Y}$ & $\mathrm{N}$ \\
\hline 2007 & CHRYSLER & Kahuna & $J C 49$ & $\mathrm{Y}$ & $\mathrm{N}$ \\
\hline 2007 & MERCEDES & C-Class Wagon & S204 & $\mathrm{Y}$ & $\mathrm{N} / \mathrm{A}$ \\
\hline 2007 & MERCEDES & C-Class Sedan & W204 & $\mathrm{Y}$ & $\mathrm{N} / \mathrm{A}$ \\
\hline 2007 & LAND ROVER & Freelander / NAS & L359/NAS & $\mathrm{Y}$ & $\mathrm{N}$ \\
\hline 2007 & MITSUBISHI & Delica & $3 \mathrm{H} 45 \mathrm{~W}$ & $\mathrm{Y}$ & $\mathrm{N}$ \\
\hline 2007 & MITSUBISHI & Lancer Evolution & GS41EVO & $\mathrm{Y}$ & $\mathrm{N}$ \\
\hline 2007 & MITSUBISHI & Lancer & GS41/GS44 & $\mathrm{Y}$ & $\mathrm{N}$ \\
\hline 2007 & CITROEN & $\mathrm{C} 5$ & $x 72$ & $\mathrm{Y}$ & $\mathrm{N} / \mathrm{A}$ \\
\hline 2007 & CITROEN & $\mathrm{C} 5$ & $x 73$ & $Y$ & $\mathrm{~N} / \mathrm{A}$ \\
\hline 2007 & CITROEN & $\mathrm{C} 5$ & $x 71$ & $Y$ & $\mathrm{~N} / \mathrm{A}$ \\
\hline 2007 & LEXUS & LX470 & $220 \mathrm{~L}$ & $\mathrm{Y}$ & $\mathrm{N} / \mathrm{A}$ \\
\hline 2007 & LEXUS & LS HYBRID & $270 \mathrm{~L}$ & $\mathrm{Y}$ & $\mathrm{N} / \mathrm{A}$ \\
\hline 2007 & LEXUS & $\mathrm{RX} 330$ & $295 \mathrm{~L}$ & $\mathrm{Y}$ & $\mathrm{N} / \mathrm{A}$ \\
\hline 2007 & LEXUS & $\mathrm{RX} 350$ & $295 L$ & $\mathrm{Y}$ & $\mathrm{N} / \mathrm{A}$ \\
\hline 2007 & TOYOTA & LAND CRUISER 100 & $215 \mathrm{~L}$ & $Y$ & $\mathrm{~N} / \mathrm{A}$ \\
\hline 2007 & TOYOTA & Highlander (Kluger) & $400 \mathrm{~L}$ & $\mathrm{Y}$ & $\mathrm{N}$ \\
\hline 2008 & BMW & 7-SERIES & F01/F02 & $\mathrm{Y}$ & $Y$ \\
\hline 2008 & ROLLS ROYCE & ROLLS ROYCE & RR03 & $\mathrm{N}$ & $\mathrm{N}$ \\
\hline 2008 & MERCEDES & C-Class Sport & X204 & $Y$ & $\mathrm{~N} / \mathrm{A}$ \\
\hline 2008 & MERCEDES & BR204 (C-class) & BR204 & $\mathrm{Y}$ & $\mathrm{N}$ \\
\hline 2008 & MITSUBISHI & Outlander & GS45X & $\mathrm{Y}$ & $\mathrm{N}$ \\
\hline 2008 & PEUGEOT & 308 & T72BK & $\mathrm{Y}$ & $\mathrm{N}$ \\
\hline 2008 & PEUGEOT & 308 & T72SW & $Y$ & $\mathrm{~N}$ \\
\hline 2008 & PEUGEOT & 308 & T76 & $Y$ & $\mathrm{~N}$ \\
\hline 2008 & TOYOTA & AVENSIS & $445 \mathrm{~L}$ & $Y$ & $\mathrm{~N} / \mathrm{A}$ \\
\hline 2008 & TOYOTA & Venza, Ace & $470 \mathrm{~L}$ & $Y$ & $\mathrm{~N}$ \\
\hline 2008 & TOYOTA & ALPHARD & $480 \mathrm{~L}$ & $\mathrm{Y}$ & $\mathrm{N} / \mathrm{A}$ \\
\hline 2008 & TOYOTA & CROWN ROYAL & $570 \mathrm{~L}$ & $\mathrm{Y}$ & $\mathrm{N} / \mathrm{A}$ \\
\hline 2008 & TOYOTA & CROWN ROYAL (Hybrid) & $585 \mathrm{~L}$ & $Y$ & $\mathrm{~N} / \mathrm{A}$ \\
\hline 2008 & TOYOTA & YARIS & $680 \mathrm{~L}$ & $Y$ & $\mathrm{~N} / \mathrm{A}$ \\
\hline 2009 & BMW & Z4 & E89 & $Y$ & Y \\
\hline 2009 & BMW & LSC & F07/LSC & $Y$ & $Y$ \\
\hline 2009 & ROLLS ROYCE & ROLLS ROYCE & RR04 NGS & $Y$ & $Y$ \\
\hline
\end{tabular}




\begin{tabular}{|c|c|c|c|c|c|}
\hline 2009 & MERCEDES & E-Class Sedan & W212 & $\mathrm{Y}$ & $\mathrm{N} / \mathrm{A}$ \\
\hline 2009 & MERCEDES & E-Class Wagon & S212 & $\mathrm{Y}$ & $\mathrm{N} / \mathrm{A}$ \\
\hline 2009 & FORD & Focus C-Max Succ. Grand & C344 & $\mathrm{Y}$ & $\mathrm{N}$ \\
\hline 2009 & FORD & Focus C-Max Succ.Compact & C344 & $\mathrm{Y}$ & $\mathrm{N}$ \\
\hline 2009 & SUZUKI & New Sedan Wagon & YW1 & $\mathrm{Y}$ & $\mathrm{N}$ \\
\hline 2009 & PORSCHE & Panamera & 970 & $\mathrm{Y}$ & $\mathrm{Y}$ \\
\hline 2009 & LEXUS & GX & 837L & $Y$ & $\mathrm{Y}$ \\
\hline 2009 & LEXUS & LF-C & $370 \mathrm{~L}$ & $\mathrm{Y}$ & $\mathrm{Y}$ \\
\hline 2009 & LEXUS & SC & $382 x$ & $\mathrm{Y}$ & $\mathrm{Y}$ \\
\hline 2009 & TOYOTA & CROWN MAJESTA & $571 \mathrm{~L}$ & $\mathrm{Y}$ & $\mathrm{N} / \mathrm{A}$ \\
\hline 2009 & TOYOTA & Prius & $590 \mathrm{~L}$ & $\mathrm{Y}$ & $Y$ \\
\hline 2009 & TOYOTA & COROLLA VERSO & $595 \mathrm{~L}$ & $\mathrm{Y}$ & $\mathrm{N} / \mathrm{A}$ \\
\hline 2009 & TOYOTA & 4-Runner/Hilux Surf & $610 \mathrm{~L}$ & $\mathrm{Y}$ & Y \\
\hline 2009 & TOYOTA & LAND CRUISER/PRADO & $611 \mathrm{~L}$ & $\mathrm{Y}$ & $\mathrm{N}$ \\
\hline 2009 & TOYOTA & Mark X & $730 \mathrm{~L}$ & $\mathrm{Y}$ & Y \\
\hline 2009 & AUDI & A8 & AU 641 & $\mathrm{Y}$ & $Y$ \\
\hline 2010 & BMW & 5-SERIES & F10 & $\mathrm{Y}$ & Y \\
\hline 2010 & BMW & 5-SERIES & F11 & $\mathrm{Y}$ & $\mathrm{Y}$ \\
\hline 2010 & BMW & 6 SERIES & F12 & $\mathrm{Y}$ & $\mathrm{Y}$ \\
\hline 2010 & CHRYSLER & Commander-Domestic & WC 75 & $\mathrm{~N}$ & $\mathrm{~N}$ \\
\hline 2010 & CHRYSLER & Grand Cherokee-Domestic & WK 2010 & $\mathrm{~N}$ & $\mathrm{~N}$ \\
\hline 2010 & CHRYSLER & Commander-Domestic & $w X$ & $\mathrm{~N}$ & $\mathrm{~N}$ \\
\hline 2010 & MERCEDES & BR207 & BR207 & $\mathrm{Y}$ & $\mathrm{N}$ \\
\hline 2010 & MERCEDES & BR212 (E-class) & BR212 & $\mathrm{Y}$ & $\mathrm{N}$ \\
\hline 2010 & FORD & Focus Succ & C346 & $\mathrm{Y}$ & $\mathrm{N}$ \\
\hline 2010 & MITSUBISHI & C-seg SUV (Concept CX) & $3 \times 45(Z C)$ & $Y$ & $\mathrm{~N}$ \\
\hline 2010 & PORSCHE & CAYENNE & E2 & $Y$ & Y \\
\hline 2010 & LEXUS & gx460 & gx - (suv) & $\mathrm{Y}$ & $Y$ \\
\hline 2010 & LEXUS & $030 \mathrm{~A}$ & $030 \mathrm{~A}$ & $\mathrm{Y}$ & $\mathrm{N} / \mathrm{A}$ \\
\hline 2010 & LEXUS & IS & 030x & $Y$ & Y \\
\hline 2010 & LEXUS & LF-A & $812 \mathrm{~L}$ & $Y$ & $Y$ \\
\hline 2010 & TOYOTA & Sienna & $580 \mathrm{~L}$ & $\mathrm{Y}$ & $\mathrm{N}$ \\
\hline 2010 & TOYOTA & Camry & $050 \mathrm{~A}$ & $\mathrm{Y}$ & $\mathrm{N}$ \\
\hline 2010 & TOYOTA & BELTA & 055X & $\mathrm{Y}$ & $\mathrm{N} / \mathrm{A}$ \\
\hline 2010 & TOYOTA & Ractis & $770 \mathrm{~L}$ & $Y$ & $\mathrm{~N} / \mathrm{A}$ \\
\hline 2010 & TOYOTA & Rav4 & $120 x$ & $Y$ & $\mathrm{Y}$ \\
\hline 2010 & TOYOTA & Vitz & $830 \mathrm{~L}$ & $Y$ & $Y$ \\
\hline 2010 & AUDI & A6 Limo & AU 571 & $Y$ & $Y$ \\
\hline 2010 & AUDI & A6 Coupe & AU 574 & $\mathrm{Y}$ & $Y$ \\
\hline 2011 & BMW & 6 SERIES & F13 & $Y$ & Y \\
\hline 2011 & BMW & 5 Series 4 DR 4WD & N/A & $Y$ & Y \\
\hline
\end{tabular}




\begin{tabular}{|c|c|c|c|c|c|}
\hline 2011 & Chevrolet & Cruze & $\mathrm{N} / \mathrm{A}$ & $\mathrm{Y}$ & $\mathrm{Y}$ \\
\hline 2011 & Chevrolet & Volt & $\mathrm{N} / \mathrm{A}$ & $\mathrm{Y}$ & $Y$ \\
\hline 2011 & Dodge & Caliber & $\mathrm{N} / \mathrm{A}$ & $\mathrm{Y}$ & $\mathrm{N}$ \\
\hline 2011 & Ford & Fusion 4 Dr Hybrid & $\mathrm{N} / \mathrm{A}$ & $Y$ & $\mathrm{~N}$ \\
\hline 2011 & Ford & Fiesta 4DR & $\mathrm{N} / \mathrm{A}$ & $\mathrm{Y}$ & $\mathrm{N}$ \\
\hline 2011 & Lexus & RX350 AWD & $\mathrm{N} / \mathrm{A}$ & $Y$ & $Y$ \\
\hline 2011 & $\begin{array}{l}\text { Mercedes- } \\
\text { Benz }\end{array}$ & C Class & N/A & $\mathrm{Y}$ & $\mathrm{N}$ \\
\hline 2011 & Scion & $\mathrm{TC}$ & $\mathrm{N} / \mathrm{A}$ & $Y$ & $Y$ \\
\hline 2011 & Toyota & Camry & $\mathrm{N} / \mathrm{A}$ & $\mathrm{Y}$ & $\mathrm{N}$ \\
\hline 2011 & Toyota & Highlander 4WD & $\mathrm{N} / \mathrm{A}$ & $Y$ & $\mathrm{~N}$ \\
\hline 2011 & Toyota & Sienna FWD & $\mathrm{N} / \mathrm{A}$ & $\mathrm{Y}$ & $\mathrm{N}$ \\
\hline 2011 & Toyota & Tundra Double Cab & $\mathrm{N} / \mathrm{A}$ & $\mathrm{Y}$ & $Y$ \\
\hline 2011 & Toyota & Venza 4WD & $\mathrm{N} / \mathrm{A}$ & $\mathrm{Y}$ & $\mathrm{N}$ \\
\hline 2011 & Toyota & Prius $5 \mathrm{HB}$ & $\mathrm{N} / \mathrm{A}$ & $\mathrm{Y}$ & $\mathrm{N}$ \\
\hline 2012 & BMW & 3 Series 4 DR RWD & N/A & $Y$ & $Y$ \\
\hline 2012 & Chevrolet & Sonic 4DR FWD & N/A & $Y$ & $Y$ \\
\hline 2012 & Chrysler & 300 4DR AWD & N/A & $\mathrm{Y}$ & $\mathrm{N}$ \\
\hline 2012 & Chrysler & Town \& Country FWD & $\mathrm{N} / \mathrm{A}$ & $\mathrm{Y}$ & $\mathrm{N}$ \\
\hline 2012 & Dodge & Charger 4DR AWD & $\mathrm{N} / \mathrm{A}$ & Y & $\mathrm{N}$ \\
\hline 2012 & Dodge & Journey 4DR 4WD & N/A & $Y$ & $\mathrm{~N}$ \\
\hline 2012 & Fiat & $5003 \mathrm{HB}$ FWD later release & N/A & $\mathrm{Y}$ & $\mathrm{N}$ \\
\hline 2012 & Lexus & ES350 4DR FWD & N/A & $\mathrm{Y}$ & $Y$ \\
\hline 2012 & Mitsubishi & Lancer 4DR FWD & $\mathrm{N} / \mathrm{A}$ & $\mathrm{Y}$ & $\mathrm{N}$ \\
\hline 2012 & Mitsubishi & Outlander Sport FWD & $\mathrm{N} / \mathrm{A}$ & $\mathrm{Y}$ & $\mathrm{N}$ \\
\hline 2012 & Scion & iQ 3HB FWD & N/A & $\mathrm{Y}$ & $Y$ \\
\hline 2012 & Subaru & Impreza 4DR AWD & N/A & $\mathrm{Y}$ & $\mathrm{N}$ \\
\hline 2012 & Toyota & 4Runner 2WD & $\mathrm{N} / \mathrm{A}$ & $\mathrm{Y}$ & Y \\
\hline 2012 & Toyota & Camry 4DR FWD & $\mathrm{N} / \mathrm{A}$ & $\mathrm{Y}$ & Y \\
\hline 2012 & Toyota & Prius Plug-in FWD & N/A & $\mathrm{Y}$ & $\mathrm{N}$ \\
\hline 2012 & Toyota & Sienna FWD & $\mathrm{N} / \mathrm{A}$ & $\mathrm{Y}$ & $\mathrm{N}$ \\
\hline 2012 & Toyota & Tundra Crew Cab 2WD & $\mathrm{N} / \mathrm{A}$ & $\mathrm{Y}$ & Y \\
\hline 2012 & Toyota & Yaris Liftback 5HB FWD & $\mathrm{N} / \mathrm{A}$ & $\mathrm{Y}$ & $\mathrm{N}$ \\
\hline 2013 & Buick & Verano FWD & $\mathrm{N} / \mathrm{A}$ & $Y$ & Y \\
\hline 2013 & Buick & Encore FWD & N/A & $\mathrm{Y}$ & $Y$ \\
\hline 2013 & Cadillac & ATS AWD & N/A & $Y$ & $\mathrm{~N}$ \\
\hline 2013 & Cadillac & XTS AWD & N/A & $Y$ & $Y$ \\
\hline 2013 & Chevrolet & Cruze FWD & $\mathrm{N} / \mathrm{A}$ & $\mathrm{Y}$ & Y \\
\hline 2013 & Dodge & Dart FWD & $\mathrm{N} / \mathrm{A}$ & $Y$ & $Y$ \\
\hline 2013 & Ford & CMAX Energi FWD & N/A & $\mathrm{Y}$ & $\mathrm{N}$ \\
\hline 2013 & Ford & CMAX HEV FWD & N/A & $Y$ & $\mathrm{~N}$ \\
\hline
\end{tabular}




\begin{tabular}{|c|c|c|c|c|c|}
\hline 2013 & Ford & Explorer 4DR AWD & $\mathrm{N} / \mathrm{A}$ & $\mathrm{N}$ & $\mathrm{Y}$ \\
\hline 2013 & Ford & Focus 4DR FWD & $\mathrm{N} / \mathrm{A}$ & $\mathrm{Y}$ & $\mathrm{N}$ \\
\hline 2013 & Ford & Focus BEV FWD & $\mathrm{N} / \mathrm{A}$ & $\mathrm{Y}$ & $\mathrm{N}$ \\
\hline 2013 & Ford & Fusion Energi FWD & $\mathrm{N} / \mathrm{A}$ & $\mathrm{Y}$ & $\mathrm{N}$ \\
\hline 2013 & Ford & Escape $4 \times 4$ & $\mathrm{~N} / \mathrm{A}$ & $\mathrm{Y}$ & $\mathrm{N}$ \\
\hline 2013 & Ford & Fusion AWD / MKZ AWD & $\mathrm{N} / \mathrm{A}$ & $\mathrm{Y}$ & $\mathrm{N}$ \\
\hline 2013 & Hyundai & Santa Fe Sport AWD & $\mathrm{N} / \mathrm{A}$ & $Y$ & $\mathrm{~N}$ \\
\hline 2013 & Lexus & ES350 FWD & N/A & $\mathrm{Y}$ & Y \\
\hline 2013 & Lexus & IS250 RWD & N/A & $\mathrm{Y}$ & Y \\
\hline 2013 & Lexus & RX350 FWD & $\mathrm{N} / \mathrm{A}$ & $\mathrm{Y}$ & Y \\
\hline 2013 & $\begin{array}{l}\text { Mercedes- } \\
\text { Benz }\end{array}$ & C Class 4WD & $\mathrm{N} / \mathrm{A}$ & $\mathrm{Y}$ & $\mathrm{N}$ \\
\hline 2013 & $\begin{array}{l}\text { Mercedes- } \\
\text { Benz }\end{array}$ & ML Class 4WD & $\mathrm{N} / \mathrm{A}$ & $\mathrm{Y}$ & $\mathrm{N}$ \\
\hline 2013 & Subaru & Impreza 4DR AWD & $\mathrm{N} / \mathrm{A}$ & $\mathrm{Y}$ & $\mathrm{N}$ \\
\hline 2013 & Toyota & Avalon FWD & N/A & $\mathrm{Y}$ & $Y$ \\
\hline 2013 & Toyota & Prius C 5HB FWD & N/A & Y & $\mathrm{N}$ \\
\hline 2013 & Toyota & Prius V SW FWD & N/A & $\mathrm{Y}$ & $\mathrm{N}$ \\
\hline 2013 & Toyota & RAV4 FWD & $\mathrm{N} / \mathrm{A}$ & $\mathrm{Y}$ & $\mathrm{N}$ \\
\hline 2013 & Toyota & Sienna & $\mathrm{N} / \mathrm{A}$ & Y & $\mathrm{N}$ \\
\hline 2013 & Toyota & Venza FWD & N/A & $\mathrm{Y}$ & $\mathrm{N}$ \\
\hline 2014 & Acura & MDX FWD & N/A & $\mathrm{Y}$ & $\mathrm{N}$ \\
\hline 2014 & Audi & A6 FWD & $\mathrm{N} / \mathrm{A}$ & $\mathrm{Y}$ & Y \\
\hline 2014 & Chevrolet & Impala 4dr FWD & $\mathrm{N} / \mathrm{A}$ & $\mathrm{Y}$ & Y \\
\hline 2014 & Chevrolet & Spark FWD & $\mathrm{N} / \mathrm{A}$ & $\mathrm{Y}$ & $Y$ \\
\hline 2014 & Dodge & Durango 2WD & N/A & $\mathrm{Y}$ & $\mathrm{N}$ \\
\hline 2014 & Jeep & Cherokee 2WD & $\mathrm{N} / \mathrm{A}$ & $\mathrm{Y}$ & $\mathrm{Y}$ \\
\hline 2014 & Jeep & $\begin{array}{c}\text { Grand Cherokee 2WD } \\
\text { early release }\end{array}$ & $\mathrm{N} / \mathrm{A}$ & $\mathrm{Y}$ & $\mathrm{N}$ \\
\hline 2014 & Jeep & $\begin{array}{c}\text { Grand Cherokee 2WD } \\
\text { later release }\end{array}$ & $\mathrm{N} / \mathrm{A}$ & $Y$ & $\mathrm{~N}$ \\
\hline 2014 & Lexus & IS250 RWD & $\mathrm{N} / \mathrm{A}$ & Y & Y \\
\hline 2014 & $\begin{array}{l}\text { Mercedes- } \\
\text { Benz }\end{array}$ & E-Class 4-DR RWD & $\mathrm{N} / \mathrm{A}$ & $\mathrm{Y}$ & $\mathrm{N}$ \\
\hline 2014 & Mitsubishi & Outlander FWD & N/A & $\mathrm{Y}$ & $\mathrm{N}$ \\
\hline 2014 & Subaru & Forester AWD & N/A & $\mathrm{Y}$ & $\mathrm{N}$ \\
\hline 2014 & Subaru & Impreza SW AWD & N/A & $\mathrm{Y}$ & $\mathrm{N}$ \\
\hline 2014 & Subaru & XV Crosstrek SW AWD & $\mathrm{N} / \mathrm{A}$ & $Y$ & $\mathrm{~N}$ \\
\hline 2014 & Toyota & Camry FWD & $\mathrm{N} / \mathrm{A}$ & Y & $\mathrm{Y}$ \\
\hline 2014 & Toyota & Highlander FWD & N/A & Y & $\mathrm{N}$ \\
\hline 2014 & Toyota & Tundra Crew Cab 2WD & N/A & $Y$ & $Y$ \\
\hline
\end{tabular}




\begin{tabular}{|cccccc|}
\hline 2014 & Toyota & Tundra Double Cab & N/A & Y & Y \\
2014 & Toyota & Corolla FWD & N/A & Y & N \\
2014 & Toyota & Prius 5HB FWD & N/A & Y & N \\
\hline
\end{tabular}

